Pacific Northwest

National Laboratory

Operated by Battelle for the

U.S. Department of Energy

\title{
Advanced Sensors and Controls for Building Applications: \\ Market Assessment and Potential R\&D Pathways
}

\author{
MR Brambley \\ D Hansen \\ P Haves \\ DR Holmberg \\ SC McDonald \\ KW Roth \\ $\mathrm{P}$ Torcellini
}

April 2005

Prepared for the U.S. Department of Energy

under Contract DE-AC05-76RL01830 


\title{
DISCLAIMER
}

This report was prepared as an account of work sponsored by an agency of the United States Government. Neither the United States Government nor any agency thereof, nor Battelle Memorial Institute, nor any of their employees, makes any warranty, express or implied, or assumes any legal liability or responsibility for the accuracy, completeness, or usefulness of any information, apparatus, product, or process disclosed, or represents that its use would not infringe privately owned rights. Reference herein to any specific commercial product, process, or service by trade name, trademark, manufacturer, or otherwise does not necessarily constitute or imply its endorsement, recommendation, or favoring by the United States Government or any agency thereof, or Battelle Memorial Institute. The views and opinions of authors expressed herein do not necessarily state or reflect those of the United States Government or any agency thereof.

\author{
PACIFIC NORTHWEST NATIONAL LABORATORY \\ operated by \\ BATTELLE \\ for the \\ UNITED STATES DEPARTMENT OF ENERGY \\ under Contract DE-AC05-76RL01830
}

Printed in the United States of America
Available to DOE and DOE contractors from the Office of Scientific and Technical Information, P.O. Box 62, Oak Ridge, TN 37831-0062;
ph: (865) 576-8401
fax: (865) 576-5728
email: reports@adonis.osti.gov

\begin{abstract}
Available to the public from the National Technical Information Service, U.S. Department of Commerce, 5285 Port Royal Rd., Springfield, VA 22161 ph: (800) 553-6847 fax: (703) 605-6900

email: orders@ntis.fedworld.gov

online ordering: http://www.ntis.gov/ordering.htm
\end{abstract}

This document was printed on recycled paper. 


\title{
Advanced Sensors and Controls for Building Applications: Market Assessment and Potential R\&D Pathways
}

\author{
MR Brambley ${ }^{(a)}$ \\ D Hansen $^{(b)}$ \\ P Haves ${ }^{(c)}$ \\ DR Holmberg ${ }^{(\mathrm{d})}$ \\ SC McDonald ${ }^{(a)}$ \\ KW $\operatorname{Roth}^{(\mathrm{f})}$ \\ P Torcellini $^{(\mathrm{e})}$
}

April 2005

Prepared for

the U.S. Department of Energy

under Contract DE-AC05-76RL01830

Pacific Northwest National Laboratory

Richland, Washington 99352

\footnotetext{
(a) Pacific Northwest National Laboratory

(b) U.S. Department of Energy

(c) Lawrence Berkeley National Laboratory

(d) National Institute of Science and Technology

(e) National Renewable Energy Laboratory

(f) TIAX, LLC
} 


\section{PREFACE}

This document provides background material on a research and development planning effort in the U.S. Department of Energy (DOE), Office of Building Technologies. It is part of a larger set of material to be used in the ongoing planning process and does not, in itself, represent the decisions or policies of DOE. This document does not represent the current DOE research agenda, nor planned research, but instead is intended to provide a point of departure for discussion of potential research options. 


\section{EXECUTIVE SUMMARY}

This document provides a market assessment of existing building sensors and controls and presents a range of technology pathways (R\&D options) for pursuing advanced sensors and building control strategies. This report is a synthesis of five white papers, each devoted to either the market assessment or the identification of R\&D options to expand the market, and resultant energy savings, from advanced building controls and sensors.

The ideas presented in these white papers were purposefully unconstrained by budget to attempt to capture the full range of potential options. As such, choosing and summarizing highlights from each of these papers, and in turn highlighting this in an Executive Summary, is quite challenging. Instead, what is contained in this Executive Summary is an overview of each chapter.

\section{Market Assessment}

The market assessment includes estimates of market potential and energy savings for sensors and control strategies currently on the market as well as a discussion of market barriers to these technologies. Contributors to this report believe that significant energy savings and increased comfort and control for occupants can be achieved with advanced technologies. An estimation of the potential market and energy savings from these advanced technologies is the subject of a follow-on market assessment by TIAX, which should be available in 2005.

\section{Technology Pathways}

The Technology Pathway is organized into four chapters:

- Current Applications and Strategies for New Applications

- Sensors and Controls

- Networking, Security, and Protocols and Standards

- Automated Diagnostics, Performance Monitoring, Commissioning, Optimal Control, and Tools.

These chapters can roughly be characterized as follows:

1. Applications to building sub-systems (e.g., lighting) and potential new applications (e.g. disaster mitigation).

2. Sensor and controls hardware including wireless devices and actuators.

3. Issues relating to the internetworking of sensors, controls, and actuators and standards and protocols required for full interoperability.

4. Tools and applications for whole building system integration, monitoring, and controls.

Each technology pathway chapter gives an overview of the technology or application. This is followed by a discussion of needs and the current status of the technology. Finally, a series of research topics is proposed. 


\section{ACKNOWLEDGMENTS}

This document is a compilation and synthesis of background material supporting the development of a research and development planning effort in advanced controls and sensors for buildings. The planning effort is led by David Hansen of the Building Technologies (BT) program within the Office of Energy Efficiency and Renewable Energy (EERE) at the U.S. Department of Energy. Representatives from DOE national laboratories, including Lawrence Berkeley National Laboratory, the National Renewable Energy Laboratory, Oak Ridge National Laboratory, and Pacific Northwest National Laboratory, as well as the National Institute for Standards and Technology (NIST) in the U.S. Department of Commerce, all contributed to the Technology Pathway chapters (Chapters 3 through 6). The market analysis chapter (Chapter 2) was developed by TIAX, LLC. All contributors are listed in the appendix. We would also like to acknowledge Theresa Gilbride of PNNL for her sharp editing skills and Andrew Nicholls of PNNL for his technical review. 


\section{ACRONYMS AND ABBREVIATIONS}

AFDD

AHU

ANSI

APAR

ASHRAE

ASTM/ISO

BACnet/IP

BAS

BCS

BT

BT

CABA

CBECS

CCC

CCTV

CEC

$\mathrm{cfm}$

CHP

$\mathrm{CO}$

$\mathrm{CO}_{2}$

DALI

DCV

DDC

DDE

DOD

DOE

DR

DSOM

EERE

EES

EIS

EMCS automatic fault detection and diagnostics

air handler unit

American National Standards Institute

AHU Performance Assessment Rules

American Society of Heating, Refrigeration and Air-Conditioning Engineers

American Society for Testing and Materials

A Data Communication Protocol for Building Automation and Control Networks

building automation system

building control system

DOE Building Technologies Program within EERE

US DOE Building Technologies Program

Continental Automated Buildings Association

Commercial Building Energy Consumption Survey

California Commissioning Collaborative

closed circuit television

California Energy Commission

cubic feet per minute

combined heat and power

carbon monoxide

carbon dioxide

digital addressable lighting interface

demand-controlled ventilation

direct digital control

dynamic data exchange

US Department of Defense

U.S. Department of Energy

demand response system

Decision Support for Operations and Maintenance software

US DOE Office of Energy Efficiency and Renewable Energy

Enron Energy Services

energy information systems

energy management control system 
EMS

ERP

ESCO

Eui

FCC

FDD

FERC

GEMnet

$\mathrm{GHz}$

GSA

GUI

Hmi

HVAC

HVAC\&R

IAQ

IBECS

ICM

ICP/IP

IEA

IEC

IEEE

IEQ

IFC

IMDS

IP

IPMVP

ISO

ISO-NE

IT

LAN

LBNL

LON

$\mathrm{MHz}$

MPM

MSTP energy management system

enterprise resource planning

energy service company

ENERGY USE INTENSITY

Federal Communications Comission

fault detection and diagnostics

Federal Energy Regulatory Commission

GSA Energy and Maintenance Newtork

gigahertz

US General Services Administration

graphical user interface

human machine interface

heating, ventilation and air conditioning

heating, ventilation and air conditioning and refrigeration

indoor air quality

integrated building environmental communications system

industrial, scientific, medial band

Internet communication protocol/internet protocol

International Energy Agency

International Engineering Consortium

Institute of Electrical and Electronics Engineers

indoor environmental quality

Industry Foundation Classes

Information Monitoring and Diagnostic System

internet protocol

International Performance Measurement and Verification Protocol

International Organization for Standardization

Independent System Operator-New England

information technology

local area network

Lawrence Berkeley National Laboratory

Networking protocol for building devices

megahertz

market penetration model

master-slave token passing 
NILM

NIST

NPV

NREL

NYISP

OA

OLE

ORNL

OTE

PECI

PID

PIER

PJM

PLC

PNNL

PV

QUAD

R\&D

$\mathrm{RH}$

SMD

SMUD

SPARK

SPP

SPP

SSL

UPS

VAV

VCBT

VOC

VPACC

VPN

WAN

WAP

WBD nonintrusive load measurement

US Department of Commerce National Institute for Standards and Technology net present value

National Renewable Energy Laboratory

New York Independent System Operator

outdoor air

object linking and embedding

Oak Ridge National Laboratory

operational test and evaluation

Portland Energy Conservation, Inc.

proportional integral derivative

Public Interest Energy Research (CEC program)

the wholesale electricity markets for the Pennsylvania, New Jersey, and Maryland region

power line carriers

Pacific Northwest National Laboratory

photovoltaic

Quadrillion $\left(10^{15}\right)$ British Thermal Units (Btus)

research and development

relative humidity

standard market design

Sacramento Municipal Utility District

Building energy simulation program

simple payback period

simple payback period

Sold State Lighting

uninterruptible power systems

variable air volume

Virtual Cybernetic Building Testbed

volatile organic compound

VAV box performance assessment control charts

Virtual private network

Wide area network

Wireless access protocol

Whole Building Diagnostician 


\section{TABLE OF CONTENTS}

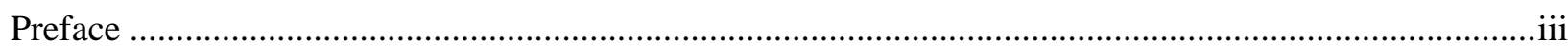

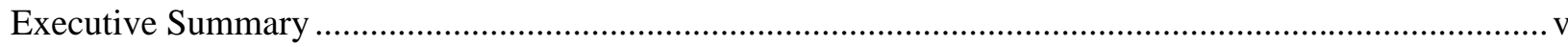

Acknowledgments............................................................................................................................... vii

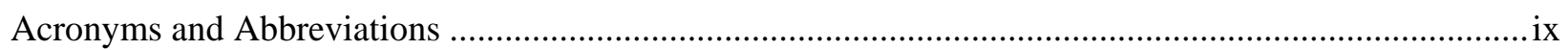

$1.0 \quad$ Introduction................................................................................................................. 1.1

2.0 The Market for Building Controls - Preliminary Assessment.........................................................2.1

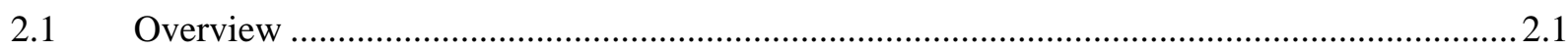

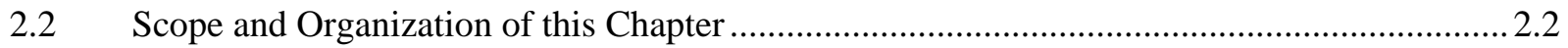

2.3 History of Computerized Building Controls............................................................................. 2.3

$2.4 \quad$ Current Building Controls Market........................................................................................... 2.4

2.4.1 Features and Functionality of Existing Building Controls Systems .................................. 2.4

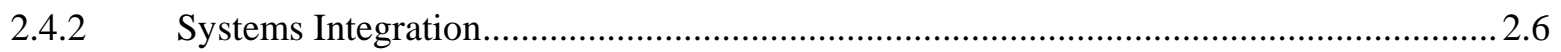

2.4.3 Intelligent Buildings ................................................................................................... 2.7

2.4.4 Prevalence of Centralized Building Controls ............................................................... 2.7

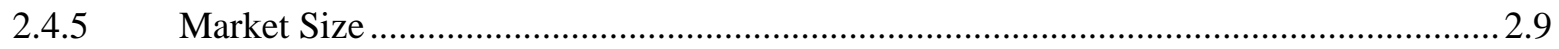

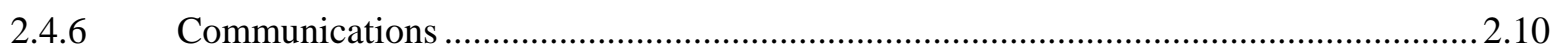

2.5 Market-Achievable Energy-Savings Potential .................................................................2.12

2.5.1 Energy Management and Control Systems (EMCS) …………………………….......... 2.14

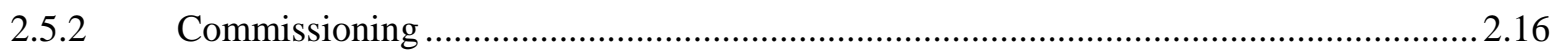

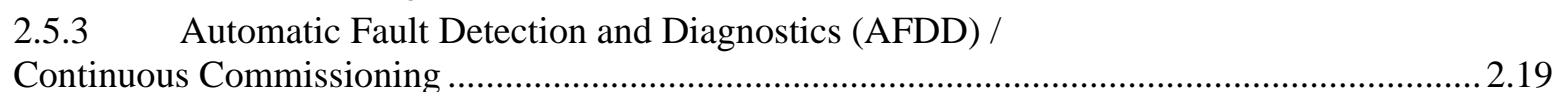

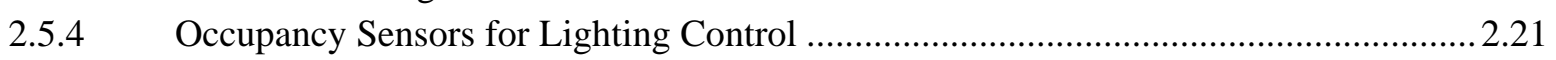

2.5.5 Photosensor-Based Lighting Control ..........................................................................2.2.2.

2.5.6 Demand Control Ventilation......................................................................................... 2.2.

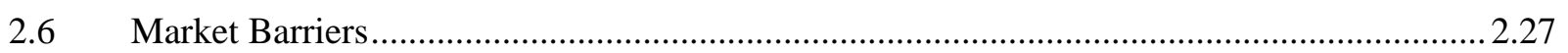

2.6.1 Minimal Concern for Energy Costs .......................................................................2.2.

2.6.2 First (Capital) Cost................................................................................................ 2.2.

2.6.3 Building Ownership and Management ...........................................................................2.29

2.6.4 Common Building Construction Paradigms ……………………………………….......2.2.

2.6.5 Building Codes and Standards .................................................................................. 2.31

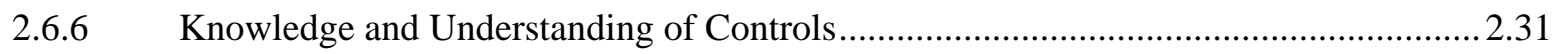

2.6.7 Experience with Existing Building Controls ................................................................. 2.32

2.7 Value Proposition for Building Controls.................................................................................2.33

2.7.1 Enhancing the Indoor Environment and Building Economic Activity ...........................2.33

2.7.2 Reducing Building Maintenance and Operations Expenses ..........................................2.34

3.0 Current Applications and Strategies for New Applications .......................................................... 3.1

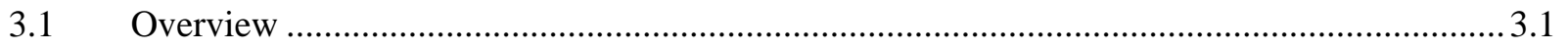

3.2 Scope and Organization...................................................................................................... 3.1

3.3 Traditional HVAC Controls .................................................................................................. 3.2

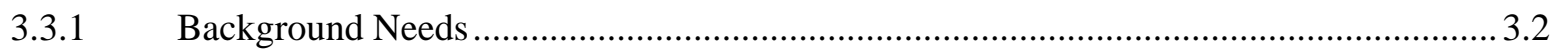

3.3.2 Current Status .................................................................................................... 3.2 


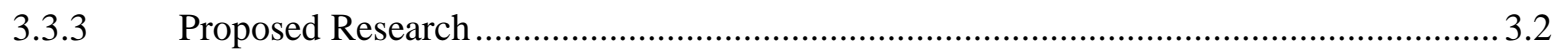

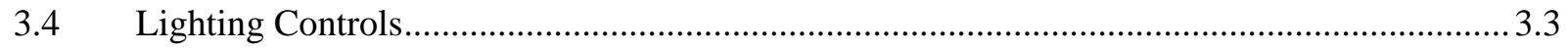

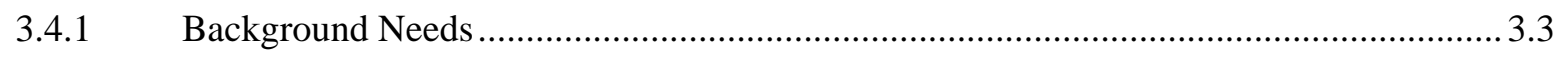

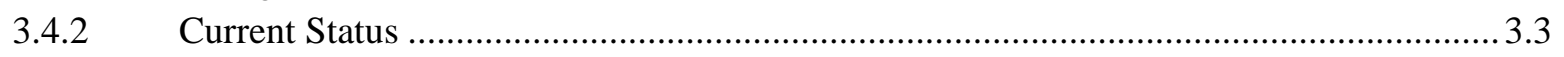

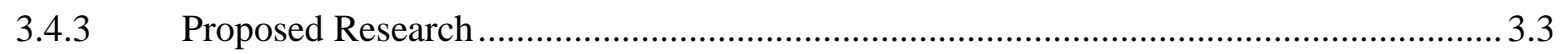

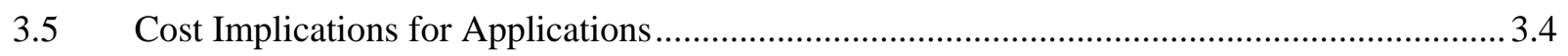

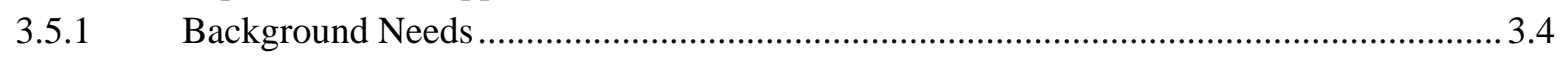

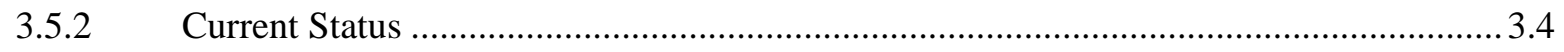

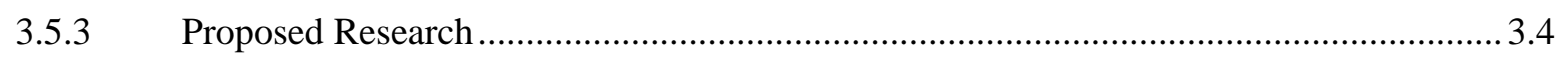

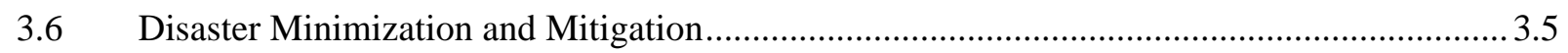

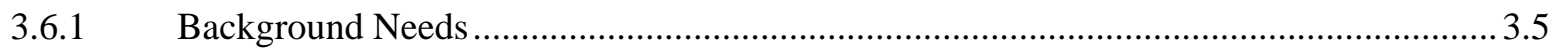

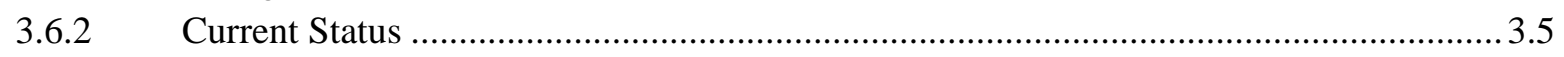

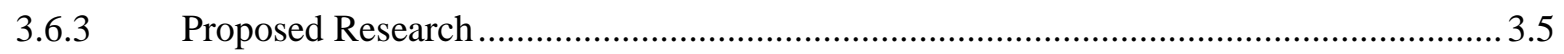

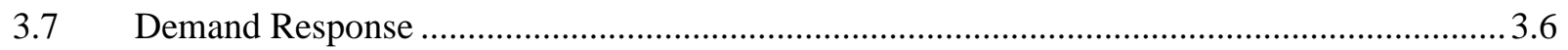

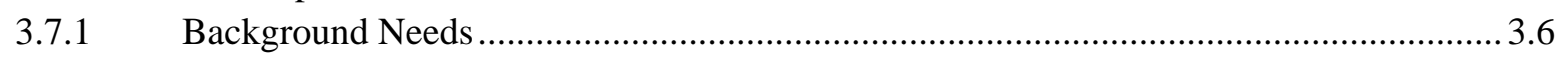

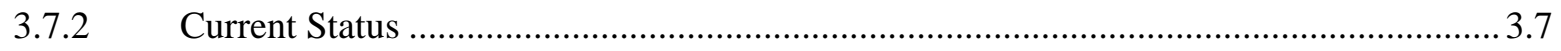

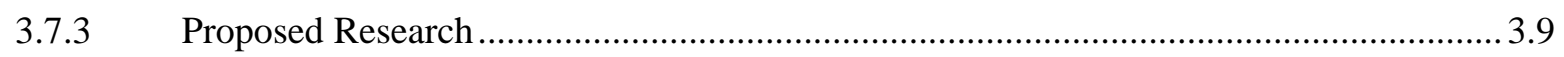

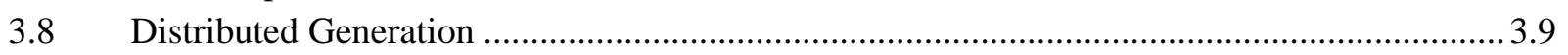

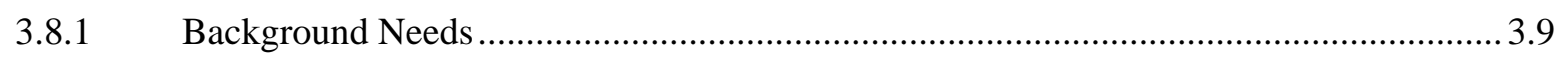

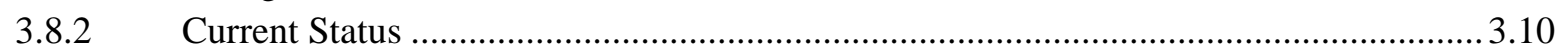

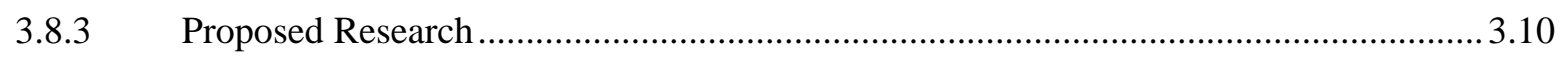

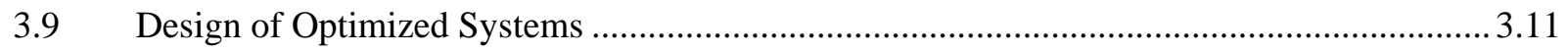

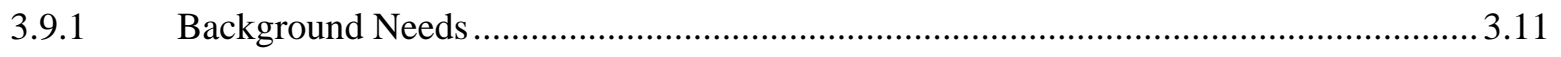

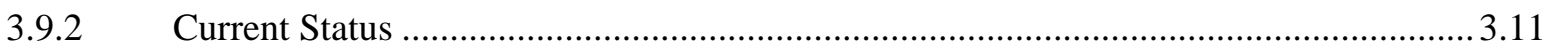

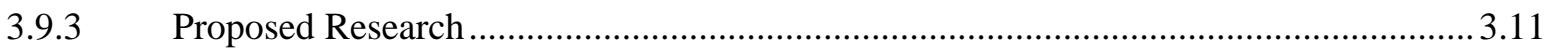

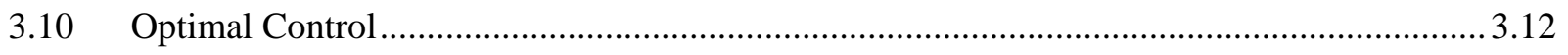

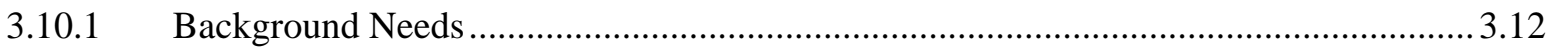

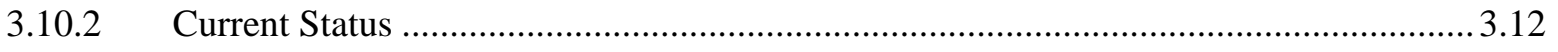

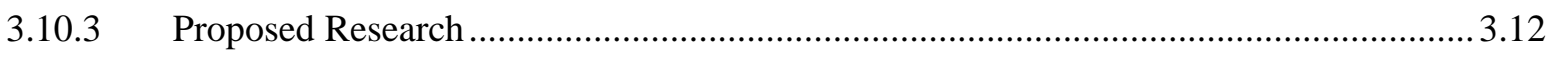

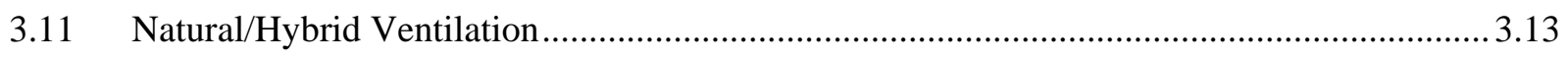

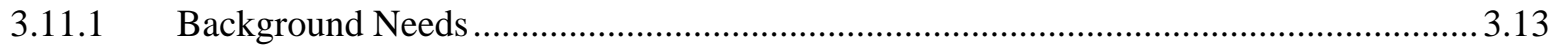

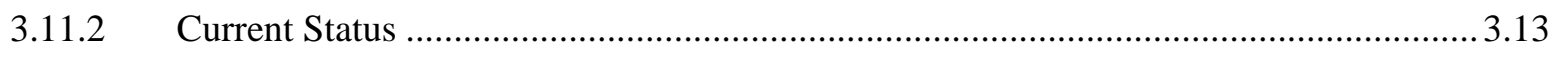

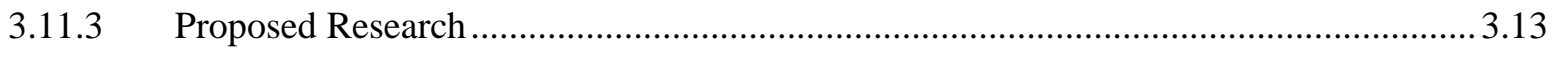

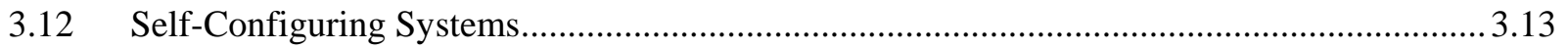

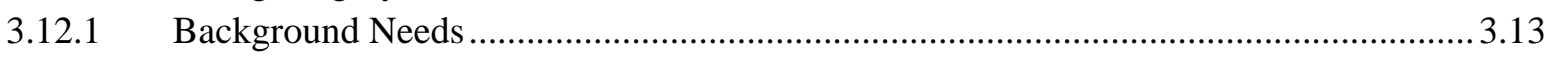

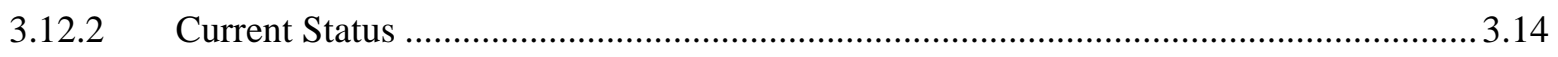

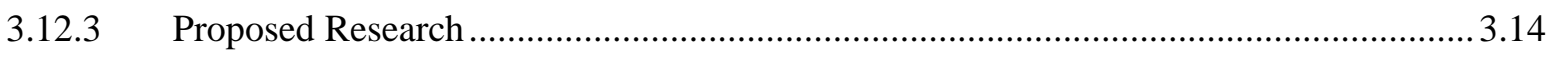

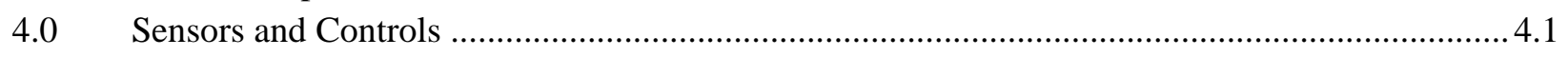

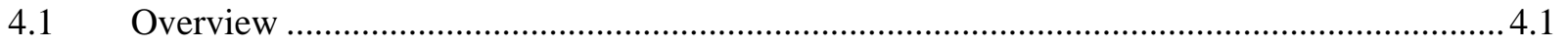

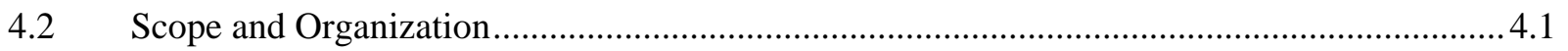

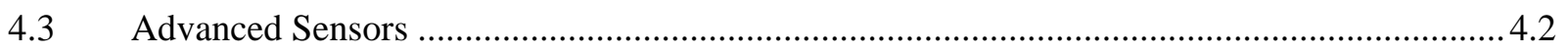

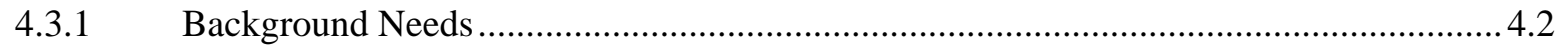

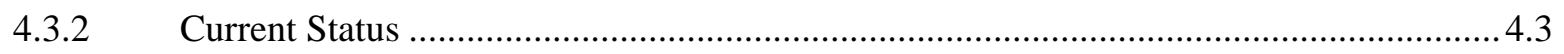

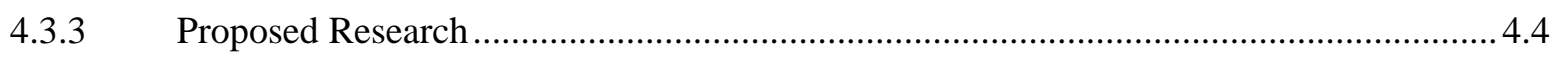




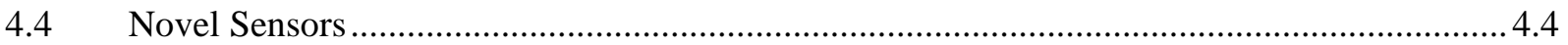

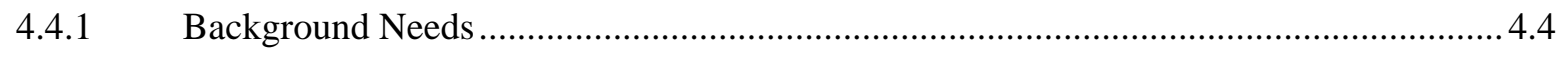

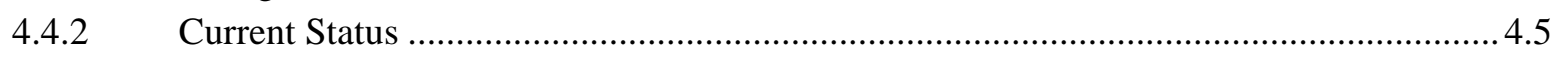

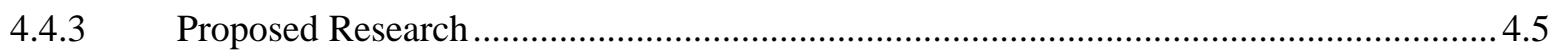

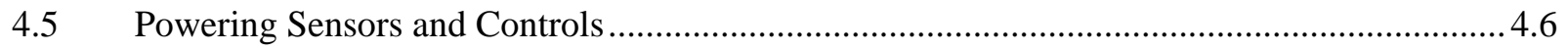

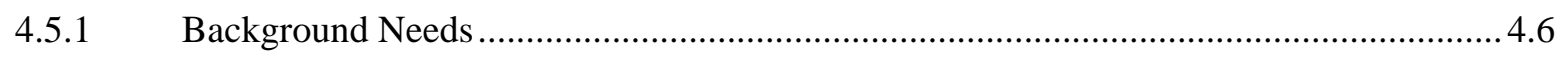

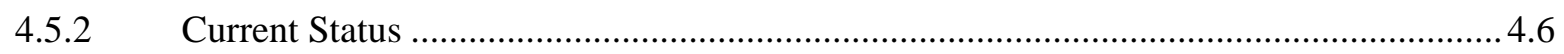

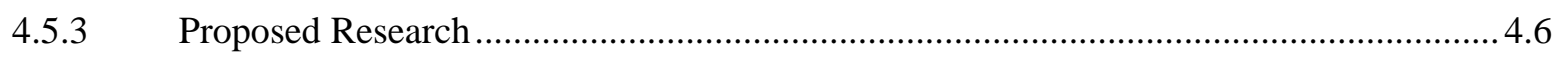

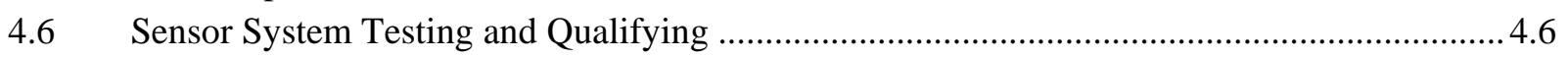

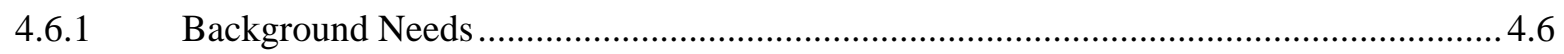

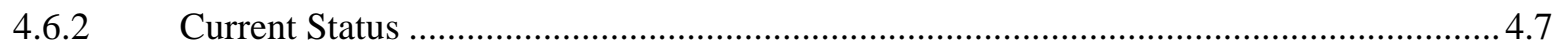

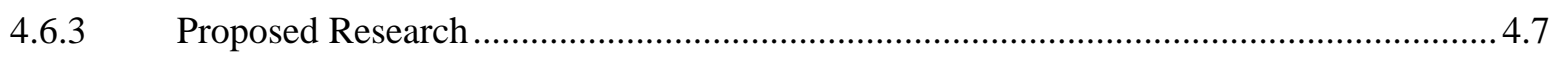

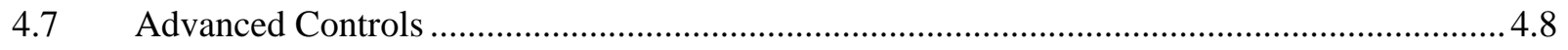

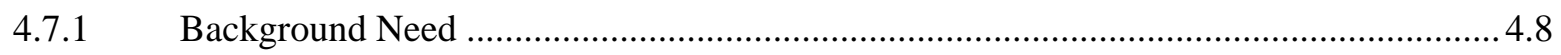

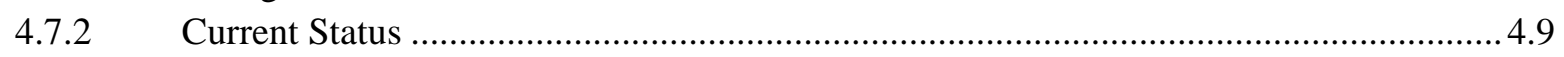

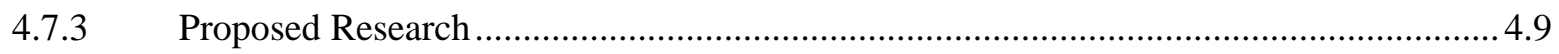

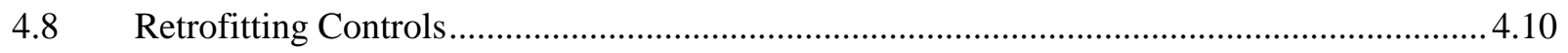

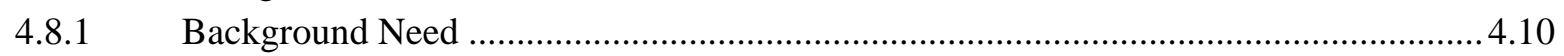

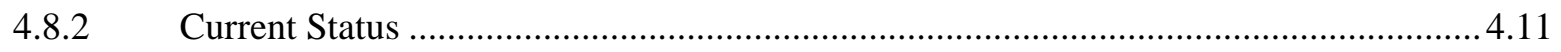

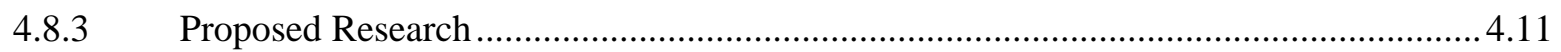

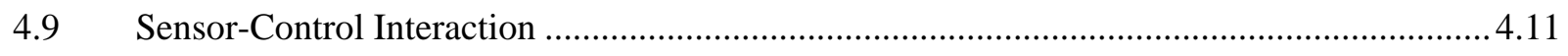

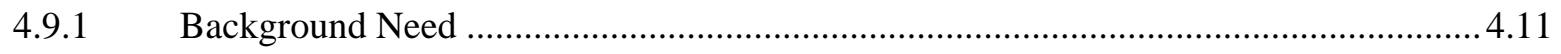

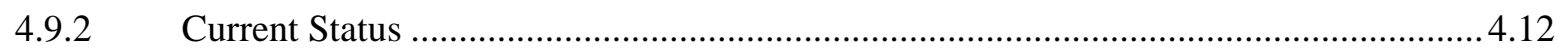

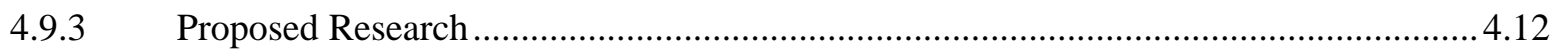

$4.10 \quad$ Sensor \& Control System Integration.............................................................................. 4.12

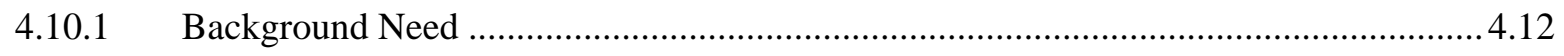

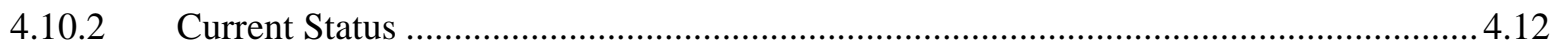

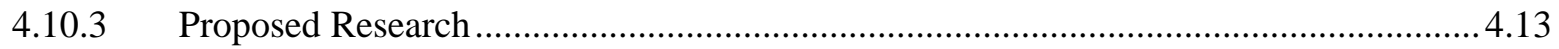

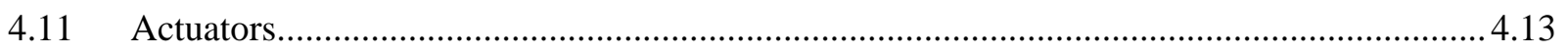

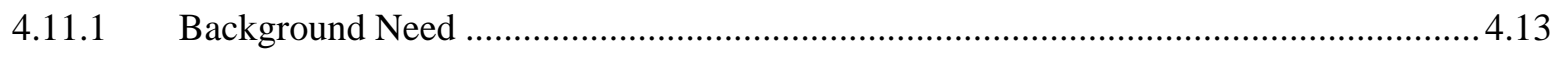

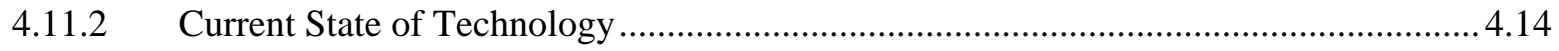

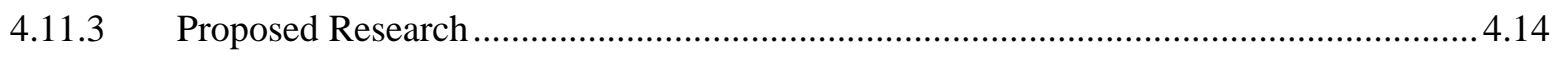

4.12 Communication Modes: Wired vs. Wireless …....................................................................15

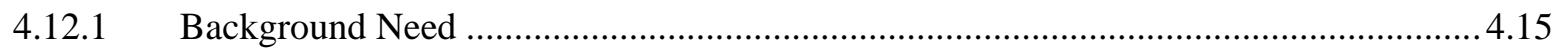

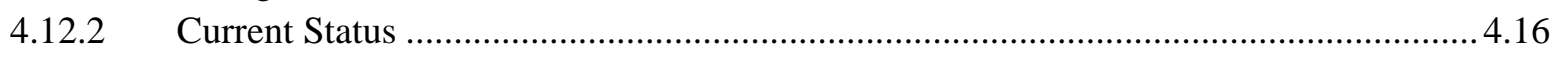

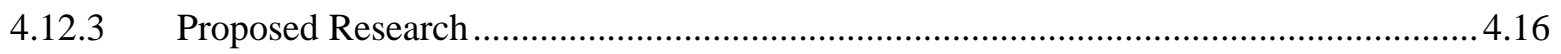

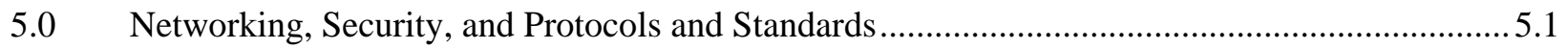

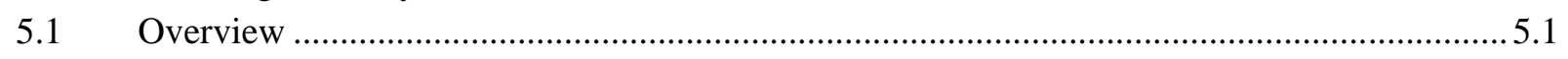

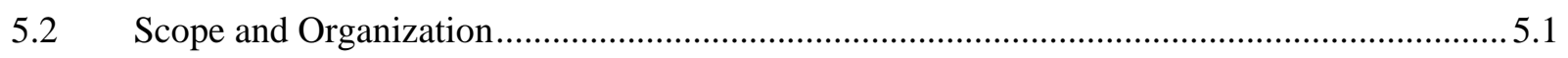

5.3 Complex Controls - Building and Grid Interactions .......................................................... 5.2

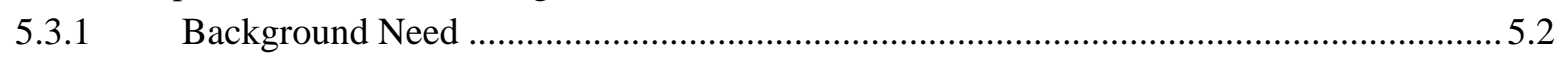

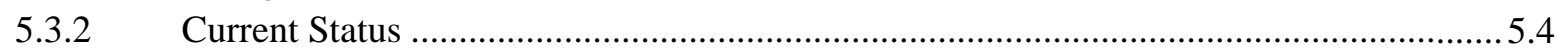

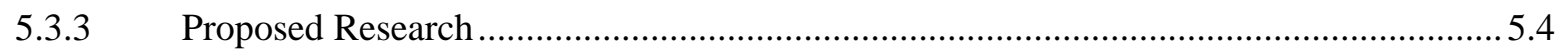

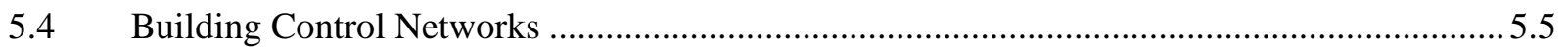




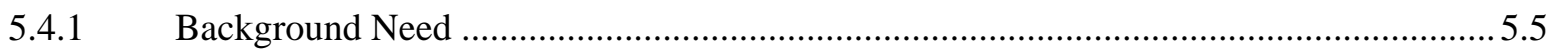

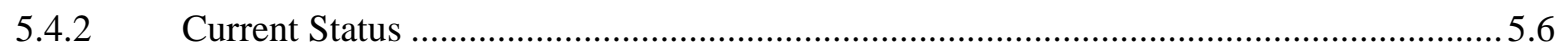

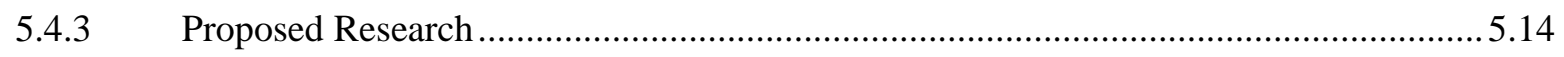

$5.5 \quad$ Building Control System Physical Security …................................................................. 5.15

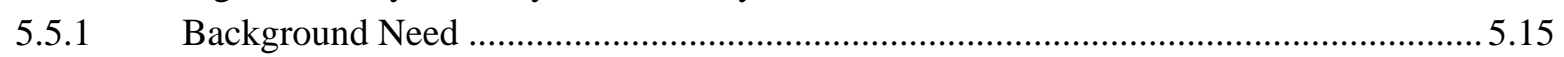

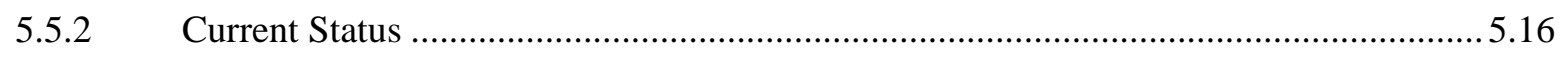

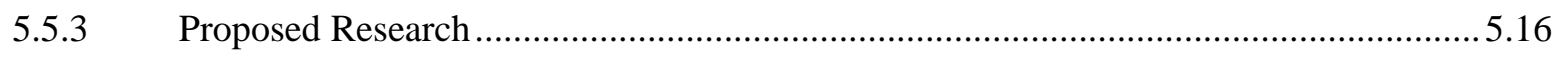

5.6 Building Control System Network Security .................................................................. 5.16

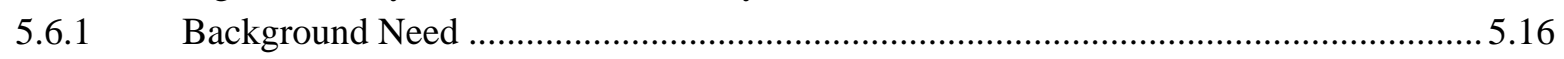

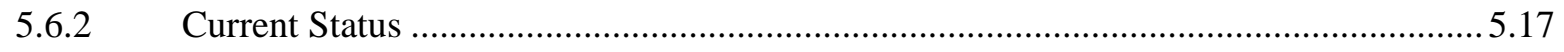

5.6.3 Proposed Research ..................................................................................................... 5.18

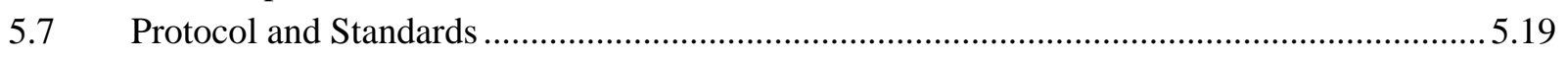

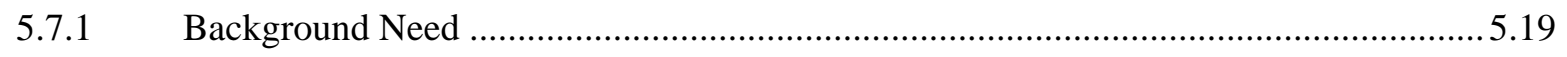

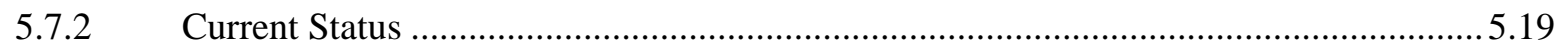

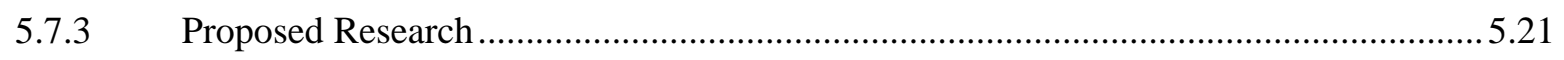

6.0 Automated Diagnostics, Performance Monitoring, Commissioning,

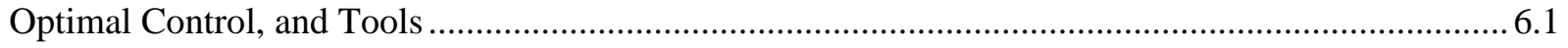

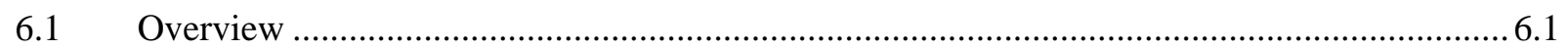

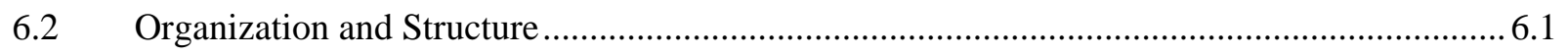

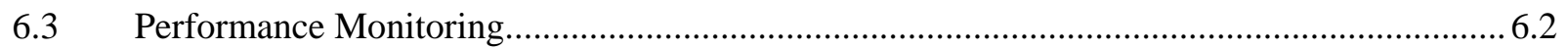

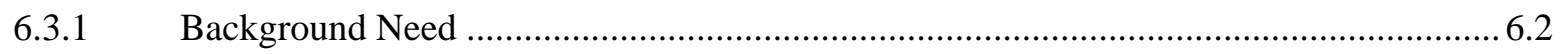

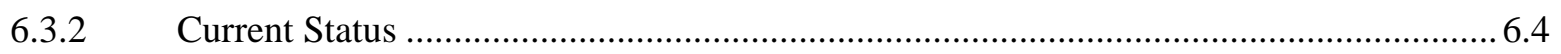

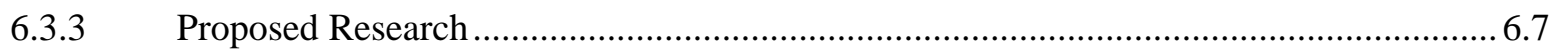

6.4 Automated Fault Detection and Diagnosis .................................................................... 6.7

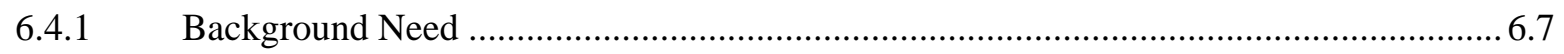

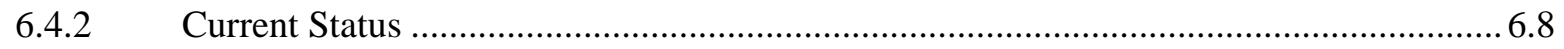

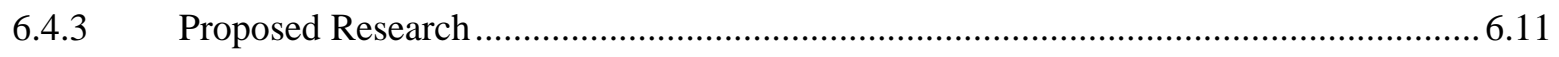

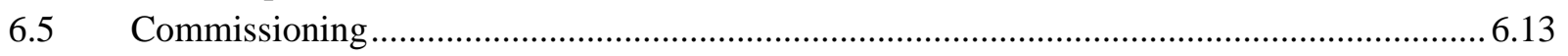

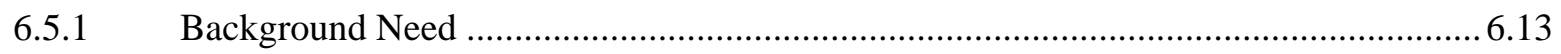

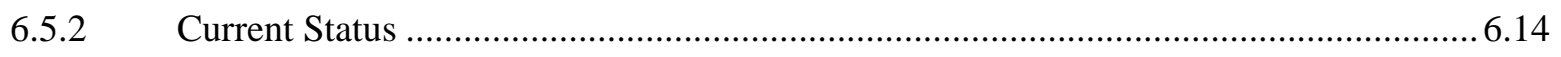

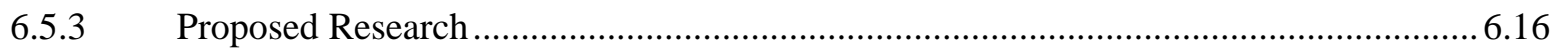

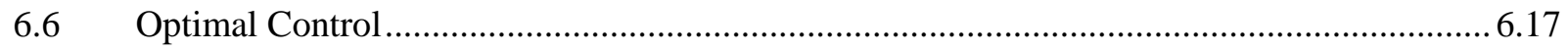

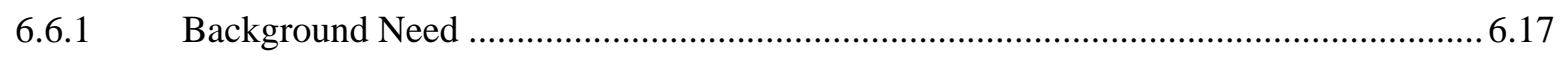

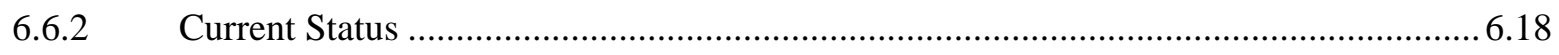

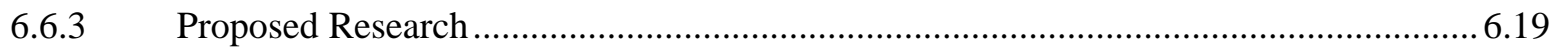

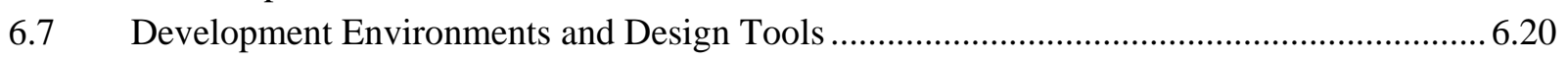

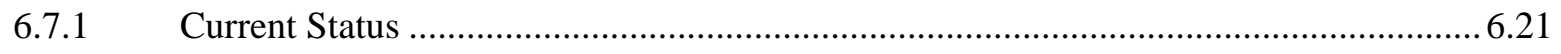

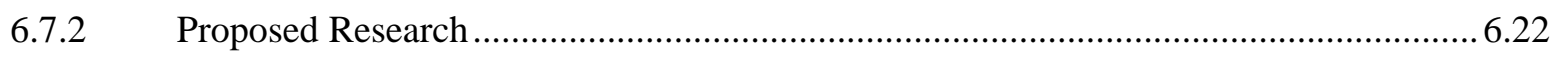

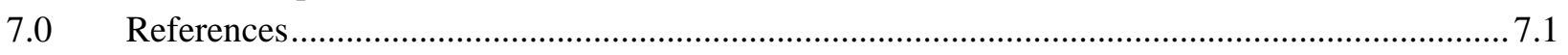

Appendix A - Lead and Contributing Authors............................................................................... A.1

Appendix B - Energy Savings Impact Estimate Calculations ............................................................. B.1 


\section{FIGURES}

Figure 1.1. Conceptual Framework and Organization ....................................................................... 1.2

Figure 2.1. Surveyed Prevalence and Usage Rates for Selected EMCS Functions (from Lowry 2002) ..2.5

Figure 2.2. Percentage of Buildings with an EMCS, by Building Size Range (from CBECS 1999) ....... 2.8

Figure 2.3. Prevalence of EMCSs by Building Type, by Percentage of Floorspace and Total Buildings

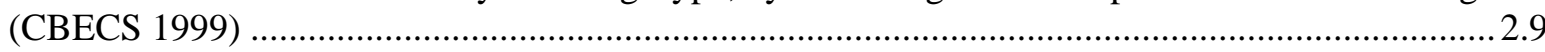

Figure 2.4. Market Size and Energy Consumption of Commercial Buildings (BTS 2002).................. 2.12

Figure 4.1. Simplified conceptual diagram of generic monitoring and control system .......................... 4.2

Figure 5.1. Networking architecture for a modern HVAC system using BACnet as the basis for communication between EMS systems ........................................................................ 5.6

Figure 5.2. Block diagram for a modern lighting control system showing the different system components and the electrical connections between them ............................................................ 5.7

Figure 5.3. Building Network on IT Backbone ................................................................................ 5.10

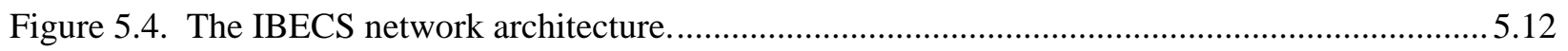

Figure 5.5. Relationship between BACnet and IBECS................................................................... 5.13 


\section{TABLES}

Table 2.1. Building Control System Functionality Classifications (based on Lowry 2002).................... 2.4

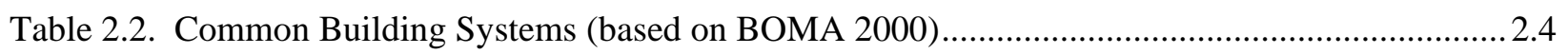

Table 2.3. General Range of Building Systems Integration (from BOMA 2000) .................................. 2.6

Table 2.4. Building Systems Most Likely to be Integrated First (from BOMA 2000) ........................... 2.7

Table 2.5. Annual U.S. Sales of Building Controls Equipment and Services (based on

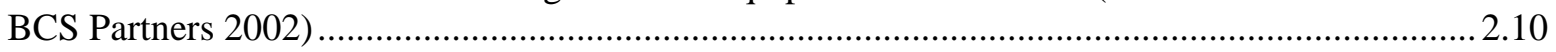

Table 2.6. Summary of Energy Savings Potential for Control Approaches ......................................... 2.14

Table 2.7. Summary of Energy Management and Control System (EMCS) Energy Savings ................2.15

Table 2.8. Summary of Commissioning Energy Savings …................................................................. 2.18

Table 2.9. Summary of AFDD/Continuous Commissioning Energy Savings......................................2.20

Table 2.10. Summary of Occupancy Sensors for Lighting Control Energy Savings.............................2.21

Table 2.11. Occupancy Sensor Energy Savings by Building Type ................................................... 2.22

Table 2.12. Summary of Photosensors for Lighting Control Energy Savings ....................................... 2.24

Table 2.13. Summary of Demand Control Ventilation (DCV) Energy Savings.................................... 2.26

Table 2.14. Breakdown of Typical Small Office Building Annual Expenditures

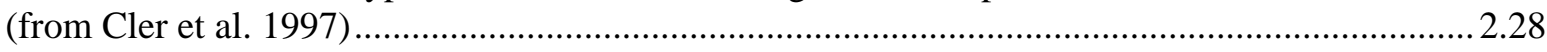

Table 2.15. Top 5 Reasons Building Owners Do Not Implement Building Systems Integration

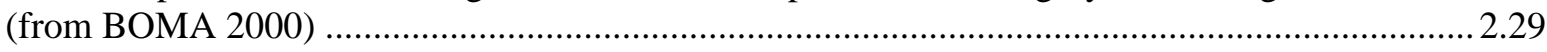

Table 2.16. Models for New Construction (based on Reed et al. 2000) ............................................... 2.30

Table 2.17. Impact of Understanding Gaps of Key Control Parties on Building Controls Energy Performance Shortfall (based on Barwig et al. 2002 and other sources) ...................................... 2.32

Table 2.18. Barriers Impeding EMCS Energy Savings and Operation, Ranked by Prevalence and Energy Impact (from Barwig et al. 2002).............................................................................. 2.33

Table 4.1. Characteristics of Two Wireless Communication Standards................................................ 4.14

Table 4.2. Features of Wireless Sensors and Controls........................................................................15 


\subsection{INTRODUCTION}

This paper represents a compilation of five separate draft white papers developed for the U.S. Department of Energy's Office of Building Technologies (DOE-BT). The white papers are

- Market Issues Surrounding the Deployment of Commercial Building Controls

- Current Applications and Strategies for New Applications

- $\quad$ Sensors and Controls

- $\quad$ Networking, Security, and Protocols and Standards

- Automated Diagnostics, Performance Monitoring, Commissioning, Optimal Control and Tools.

These papers attempt to define both the opportunity (market potential and barriers, current and future applications) and potential pathways to achieve that potential through targeted research and development (R\&D).

Conceptually, a building automation system (BAS) can be divided into four areas: applications, hardware, communications, and oversight, which interrelate as shown on Figure 1.1. The chapters of the advanced control research plan can likewise be divided into these same areas. Beyond the market analysis (Chapter 2), Chapter 3 deals with current and potential applications. Chapter 4 discusses hardware including sensors and controls. Chapter 5 deals with networking issues including standards and protocols. Chapter 6 discusses the actual process (tools and approaches) for overseeing building control including monitoring, commissioning, and diagnostics. Chapter 7 is references. Appendix A lists the lead and contributing authors for each section. Appendix B is energy savings impact estimate calculations.

In the development of these white papers, the potential R\&D options were unbounded. That is, they were developed without consideration of resource and cost constraints. This was intentional as the goal was, and is, to explore the full range of options. In addition, it is recognized that some of these R\&D options may be outside the purview of DOE.

This document should be considered solely as background material for R\&D planning. It does not, in itself, represent decisions or policies of the US DOE. This document does not describe the current DOE research agenda, nor is it a record of decision for future planned research. Instead, this paper is intended to present for consideration a broad range of potential research options (technology pathways) for consideration by DOE or others. 


\section{Conceptual Framework and Organization}

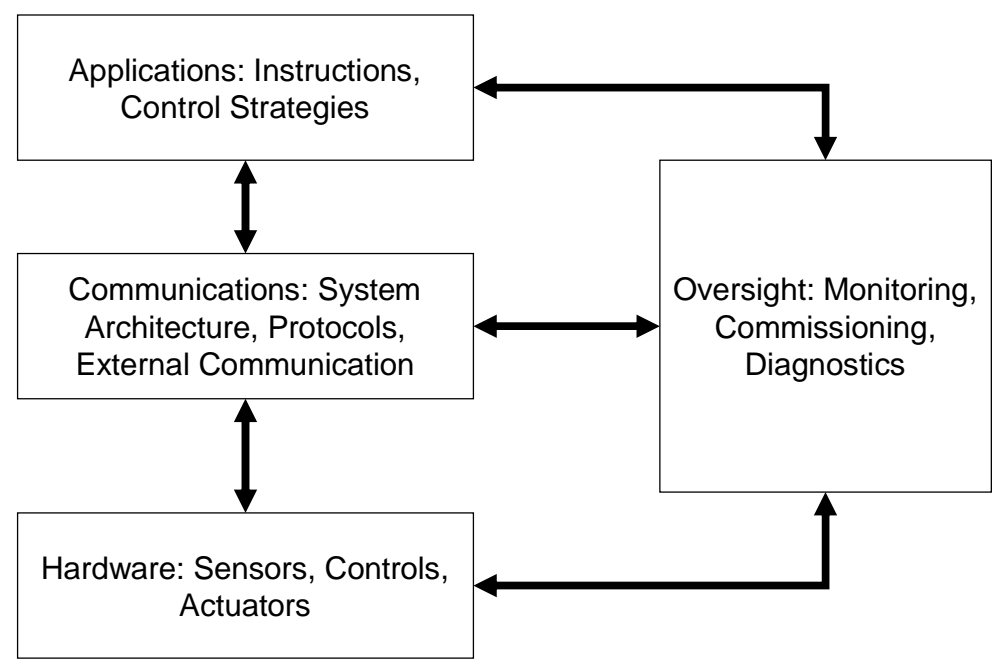

Figure 1.1. Conceptual Framework and Organization 


\subsection{THE MARKeT FOR BUILDINg CONTROLS - PRELIMINARY ASSESSMENT}

\subsection{Overview}

Commercial buildings are a significant and growing consumer of America's energy resources. America's 4.7 million commercial buildings span a great variety of functions, sizes, operating schedules and types, from large "24/7" hospitals to small retail stores. Providing the necessary energy services in these buildings (lighting, comfort, fresh air, cooking, and power for computers and other equipment) required 17.4 quadrillion Btu (quads) in 2002, 18\% of the Nation's annual energy use (DOE 2004a). Commercial buildings also constitute the most electric-intensive sector in the country; $76 \%$ of their energy services are provided by electricity, and they consume $35 \%$ of the Nation's total electricity.

Building controls have the technical potential to reduce U.S. commercial building HVAC and lighting energy consumption by about one quad of primary energy annually, or roughly $6 \%$ of current total use. In addition, many offer significant peak demand reduction potential. However, the energy savings estimates of the control approaches analyzed, particularly the market-achievable energy savings, have very large uncertainties because of wide ranges in expected market penetration based on the energysavings simple payback period. In large part, these uncertainties stem from limited data availability and narrow data applicability. Currently, most advanced controls approaches have a very small market share. Of the approaches studied, energy management control systems (EMCSs) have the largest market share, serving about one-third of lit commercial building floorspace. The current market penetration of EMCS exceeds that predicted solely by energy economics, which points to the importance of non-energy benefits in the decision to purchase an EMCS.

Advanced building controls face first-cost and several non-economic barriers to realizing greater market penetration. In owner-occupied buildings, owners typically pay little attention to energy expenditures and savings potential because building energy expenses account for a diminutive fraction of total building economic activity (e.g., about $1 \%$ of total office expenses). ${ }^{1}$ Furthermore, if energy efficiency investments are considered, they often compete with attractive investments in core business activities. In leased buildings the owner often passes energy expenses through to the tenant, giving the owner little - if any - incentive to reduce energy expenses. Generally, the owner will only invest in measures that contribute to his ultimate goal, i.e., realizing the highest rate of return possible.

Current building paradigms also impede deployment of advanced controls. The most common approach, design/build, focuses on completing buildings quickly and inexpensively and has a sequential design process that makes extensive use of prior and standard designs, resulting in a bias against innovative and relatively unproven approaches. For instance, this impedes integration of building systems, an approach that requires significant information sharing and integration between all parties designing and installing the different building systems. Finally, a general lack of knowledge about and understanding of building controls by most parties (owners, operators, designers, etc.) works against consideration, installation, and

\footnotetext{
${ }^{1}$ Editor's note: Office buildings are just one class of commercial buildings. Commercial buildings include hospitals, restaurants, hotels, warehouses and all other non-residential structures except high-rise (greater than three stories) residential.
} 
use of more sophisticated buildings controls, while also compromising the functional and energy savings efficacy of existing controls.

Somewhat paradoxically, the ability of building controls to provide non-energy benefits to building owners and occupants holds the key to greater future market penetration and national energy savings. Building controls that improve indoor environmental quality (IEQ) can greatly improve their value to the building occupants, primarily by increasing the economic activity in the building, e.g., office worker productivity or retail sales. For instance, roughly a $2 \%$ increase in the productivity of office building occupants has the same economic impact as eliminating all building maintenance and energy expenditures - employee salaries simply account for a much, much larger portion of total building expenses. At present, however, more rigorous documentation of the linkage between IEQ, building controls, and building economic activity is needed to make a convincing case to building owners and operators. This will also reduce the perceived risk of investing in IEQ-enhancing controls. Similarly, to the degree that superior IEQ increases employee retention or improves the perceived stature of a building, building controls can add value. The historical reasons for the installation of an EMCS, reduction of building operation and maintenance expenses and energy expenditures, remain attractive value propositions for advanced building controls if they can achieve these goals in a cost-effective manner.

Building controls appear to have the potential to significantly reduce commercial building energy consumption in the United States, but, at present, building controls have probably realized only a fraction of their national energy-savings potential. Overall, Energy Management Control Systems (EMCSs) manage only about one-third of commercial building floorspace ( $10 \%$ of all buildings), while more advanced control approaches ${ }^{1}$ have an even smaller market share. Building operators appear to exploit only a fraction of available EMCS functionality and, hence, energy savings.

\subsection{Scope and Organization of this Chapter}

This chapter assesses the current state of the building controls market, as well as the general magnitude of the energy savings potential of selected building control approaches. Some building controls operate effectively in a stand-alone mode, e.g., occupancy sensor-based lighting control. Centralized building controls, on the other hand, operate on a building-wide scale and require communication between the different sensors, actuators, and controllers to affect appropriate control actions. In the context of this report, building controls refers to all controls used to control energy-consuming building systems, while centralized building controls denote controls that are centrally coordinated at a single location, such as those operated through an EMCS. The chapter seeks to answer three questions:

1. To what extent are building controls, notably centralized building controls, used in commercial buildings today?

2. What is the approximate national energy savings potential of building controls, from increased deployment of building controls and more effective use of existing controls, relative to the current building stock?

\footnotetext{
${ }^{1}$ For the purpose of this paper, “advanced controls” include but are not limited to integrated building systems, automated fault detection and diagnostics (including continuous building commissioning), and advanced control algorithms (adaptive, fuzzy, nonlinear, etc.).
} 
3. What barriers impede greater and more effective use of building controls, especially "whole building" controls, and what are the potent drivers that could increase their market penetration?

Following a brief introduction to building EMCSs, there is a discussion of the prevalence and functionality of current building controls, the size of the building controls market, and the important role of communications in centralized building controls. The subsequent section develops quantitative estimates for the technical and market-achievable energy savings potential of several building controls approaches while noting important data gaps. ${ }^{1}$ The next section examines key barriers common to most building controls that arise from the commercial buildings market and other factors, as well as the apparent reasons that existing building controls fall short of realizing their energy savings potential. The final section discusses value propositions for building controls that could enhance their market penetration.

\subsection{History of Computerized Building Controls}

Large centralized building computerized control systems first appeared in the 1960s. These evolved from industrial process control systems into mini-computer-controlled systems deployed in the late 1960s. Initially, they appeared in only the largest new buildings where the first cost of the system could be broadly amortized and reductions realized in buildings operation and maintenance staff (BCS Partners 2002).

Energy became a significant concern in the early- and mid-1970s as a result of the oil embargoes. Energy cost pressures increased the market share of EMCSs. In addition, the functionality of EMCSs expanded, incorporating energy-saving features such as separate day and night schedules for HVAC and lighting, and demand control (BCS Partners 2002).

Early systems used pneumatic communications and controls. In the early 1980s, direct digital controls (DDC) were introduced to the building controls market. The "Big 3" - Johnson Controls, Honeywell, and Siemens - came to dominate this market ( $\sim 80 \%$ market share in the mid-1980s) with competing, proprietary systems (BCS Partners 2002).

The move to electronic-based DDC, enabled by the dramatic increases in computing power and the concurrent miniaturization and cost decrease of electronic components, lowered barriers to entry and placed increased emphasis on the technical qualities and capabilities of these systems. Software controllers began to supplant hard-wired control logic. This enabled many smaller players to enter the market and eroded the market share of established manufacturers (BCS Partners 2002).

In the 1990s, interoperability of systems became a significant concern of end-users. As such, the market began to move toward open protocols such as BACNet ${ }^{\mathrm{TM}}$ and LonTalk ${ }^{\circledR}$ (BCS Partners 2002). User interaction with building controls also changed with the development of more user-friendly graphical interfaces. These included web-based interfaces with enhanced graphics and the possibility of costeffective control from remote locations (e.g., via the Internet).

\footnotetext{
${ }^{1}$ Technical Energy Savings Potential refers to the expected energy savings if the energy saving approach were applied to all potential floorspace not currently served by the approach, while market-achievable energy savings potential denotes the estimated energy savings taking into account the economic and other market factors relevant to that approach.
} 


\subsection{Current Building Controls Market}

In the context of this report, building controls refers to the control of HVAC and lighting systems and equipment. Building control functionality can be further sub-divided by functionality into several different classifications (see Table 2.1). Many of these control functions are relevant to control at either the central (i.e., EMCS) or equipment/system level.

Table 2.1. Building Control System Functionality Classifications (based on Lowry 2002)

\begin{tabular}{|l|l|}
\hline $\begin{array}{l}\text { Building Control } \\
\text { Functionality Classification }\end{array}$ & Examples \\
\hline Plant Control & Space temperature control, boiler sequencing \\
\hline Plant Maintenance & $\begin{array}{l}\text { Fault reporting/alarming, filter conditioning monitoring, } \\
\text { equipment “run-time” monitoring }\end{array}$ \\
\hline Energy Saving & $\begin{array}{l}\text { HVAC/lighting scheduling, demand limitation, building night } \\
\text { purge }\end{array}$ \\
\hline Recording & Energy metering, energy use monitoring (e.g., gas, electric, oil) \\
\hline
\end{tabular}

In turn, HVAC and lighting control are two types of building systems (see Table 2).

Table 2.2. Common Building Systems (based on BOMA 2000)

\begin{tabular}{|l|l|}
\hline Building Systems & Functionality \\
\hline Access Control & Building access systems, e.g. key cards \\
\hline Fire /Life Safety & $\begin{array}{l}\text { Fire detection and alarming, fire response, fire } \\
\text { suppression }\end{array}$ \\
\hline HVAC & Climate control (temperature, humidity), ventilation \\
\hline Lighting & Lighting control \\
\hline Security & $\begin{array}{l}\text { Building alarm monitoring, surveillance cameras (closed- } \\
\text { circuit TV, a.k.a. CCTV) }\end{array}$ \\
\hline Vertical Transport & Elevator and escalator control \\
\hline
\end{tabular}

\subsubsection{Features and Functionality of Existing Building Controls Systems}

Building controls, particularly centralized building controls that are part of an EMCS, can perform a wide range of functions. While approaches to building controls have evolved dramatically over the past two decades, it is not clear that functionality has undergone a similar evolution. Actual data on the degree of building control system functionality are difficult to obtain.

Specifically, it is difficult to characterize and differentiate between the range of potential functions available in existing EMCS installations and the range of functionality actually exploited. Currently, most commercial buildings of all sizes likely have some degree of the basic control functionality found in an EMCS. For example, according to respondents of the latest EIA CBECS survey, about $80 \%$ of commercial building operators vary their building temperature setpoints for heating and cooling during unoccupied periods. ${ }^{1}$ In contrast to the probability of having an EMCS installed, the utilization of occupancy-based setback varies little with building size, presumably because most buildings have (and use) some sort of a thermostat with setback capability (CBECS 1999).

\footnotetext{
${ }^{1}$ Editor's note: These are survey responses, not audited behaviors.
} 
Studies suggest that building operators tend to use only a fraction of possible EMCS functionality, thus limiting the performance gains (Energy Design Resources 1998; Hall 2001; Barwig et al. 2002; Lowry 2002). A survey by Lowry (2002) provides some insight into the general range of available EMCS functionality and the degree to which building operators exploit available functions (see Figure 2.1). ${ }^{1}$ The results of the survey seem to support the above referenced studies.

All of the EMCSs had been updated in the last 12 years, with more than 70\% updated in the last two years. As shown in Figure 2.1, most EMCSs have, and most operators make use of, basic plant control functions. Nonetheless, many EMCSs have only a limited number of more sophisticated functions such as night purge (pre-cooling) and peak demand limiting. Furthermore, the relatively low levels of lift monitoring, security management and fire management functionality suggest that most EMCSs are not integrated with other building systems. The survey seems to support the notion that many EMCSs do not make use of a significant portion of their potential functionality.

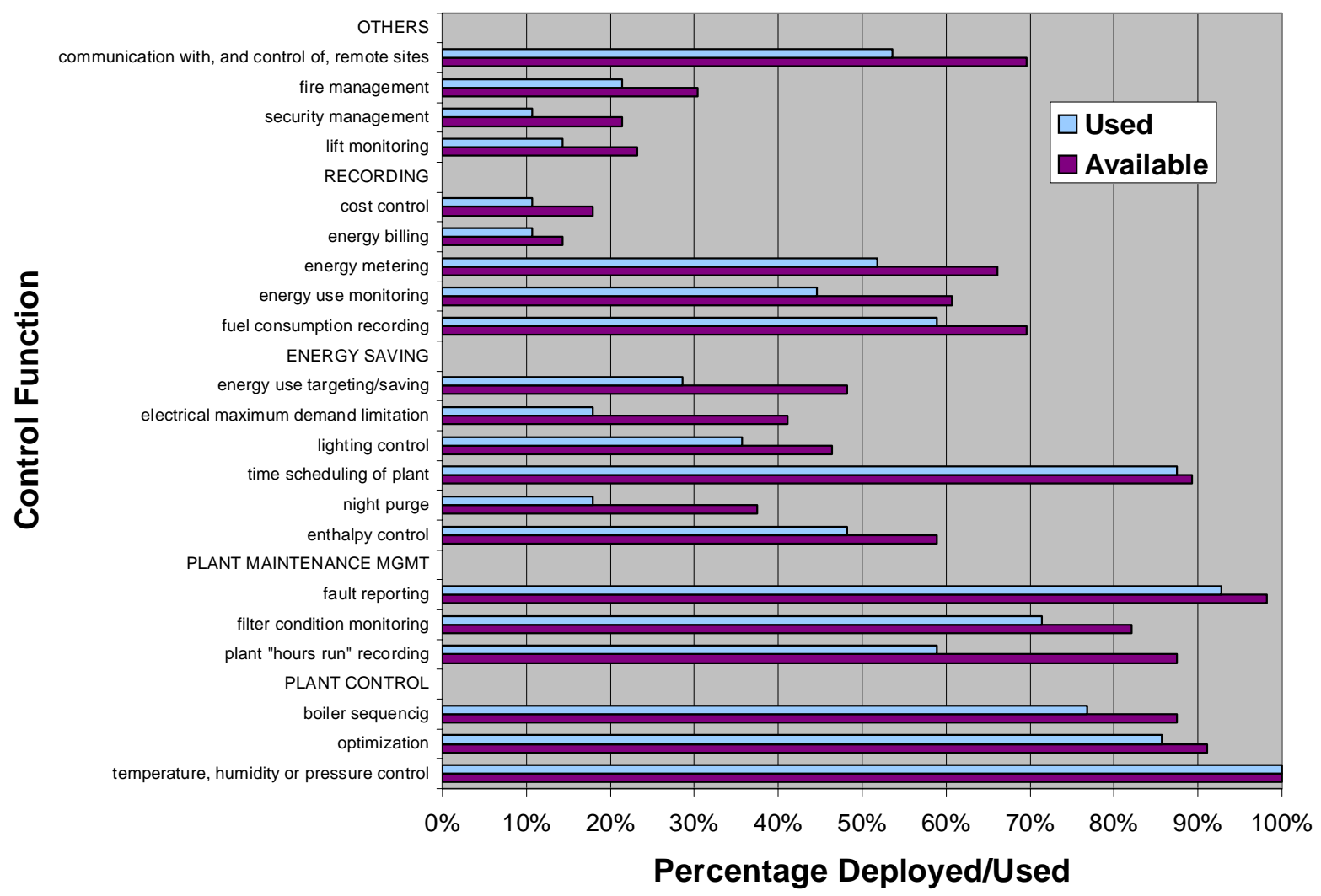

Figure 2.1. Surveyed Prevalence and Usage Rates for Selected EMCS Functions (from Lowry 2002)

\footnotetext{
${ }^{1}$ The survey includes only British building service engineers, is relatively small (56 respondents), and is based on operators enrolled in a master's degree distance-learning course in building controls.
} 


\subsubsection{Systems Integration}

Beyond energy management, centralized systems offer the potential for increased data and information sharing between different sensors and building systems. This increased information sharing can, in turn, enable increased functionality of all systems through systems integration. For example, the building access system could enable or disable vertical transport systems as well as turn on or off HVAC and lighting systems, e.g., when people arrive at work instead of at a pre-set time (McGowan 1995). Table 2.3 presents a potential range of building systems integration. The third level of integration, "Electronic Communications between Controls in a System,” is typical of most new EMCSs at present.

Table 2.3. General Range of Building Systems Integration (from BOMA 2000)

\begin{tabular}{|l|l|}
\hline Integration & Description \\
\hline Hard-Wired & Wired connection between controls \\
\hline $\begin{array}{l}\text { Electronic Communications between } \\
\text { Controls in a System }\end{array}$ & $\begin{array}{l}\text { Selected components of a common system communicate with } \\
\text { each other; developed in proprietary controls/system context }\end{array}$ \\
\hline $\begin{array}{l}\text { Electronic Communications between } \\
\text { Controls in Different Systems }\end{array}$ & $\begin{array}{l}\text { Sharing of information between different buildings systems; } \\
\text { around since mid-1990s }\end{array}$ \\
\hline $\begin{array}{l}\text { Building Systems Communicating with } \\
\text { Management System }\end{array}$ & $\begin{array}{l}\text { Front-end system integrates and shares inputs from different } \\
\text { systems (potentially with different communication protocols) }\end{array}$ \\
\hline $\begin{array}{l}\text { Enterprise-Wide Electronic Sharing of } \\
\text { Information between Controls }\end{array}$ & $\begin{array}{l}\text { Information potentially shared between most building system } \\
\text { components }\end{array}$ \\
\hline
\end{tabular}

A Building Owners and Managers Association (BOMA) survey explored the degree to which building owners have and are considering applying systems integration (BOMA 2000). The survey found the following:

- $50 \%$ of owners responding had invested in systems integration for at least some portion of their buildings

- $\quad 75 \%$ had systems integration projects planned for "very near future"

- "Virtually all” firms who had made prior investments in building integration planned future projects involving building integration

- In general, firms owning more buildings were more likely to have invested in building integration

- Cost was the primary driver in decisions to invest or not invest in systems integration, with reduced operating costs most important for those deciding to integrate systems and installed cost most important for those who decided not to pursue systems integration.

The survey clearly points out that building owners have an interest in integrating building systems if they feel confident that integrated building systems will provide real value, e.g., reduced operating costs. The same survey also found that building owners were most likely to integrate HVAC and fire safety systems on a building-wide scale first (see Table 2.4). 
Table 2.4. Building Systems Most Likely to be Integrated First (from BOMA 2000)

\begin{tabular}{|l|c|}
\hline System & $\mathbf{\%}$ \\
\hline HVAC & $91 \%$ \\
\hline Fire Safety & $77 \%$ \\
\hline Electrical Monitoring / Management & $50 \%$ \\
\hline Access Control & $45 \%$ \\
\hline Power Consumption & $45 \%$ \\
\hline Life Safety & $36 \%$ \\
\hline Lighting Controls & $36 \%$ \\
\hline CCTV & $27 \%$ \\
\hline Lighting Management & $27 \%$ \\
\hline Vertical Transportation & $18 \%$ \\
\hline
\end{tabular}

\subsubsection{Intelligent Buildings}

“Intelligent” buildings have received attention due to their enhanced potential to reduce energy use and operations and maintenance expenses, while improving the indoor environment. To achieve this potential, these systems typically employ a wide range of sensors (e.g., temperature, $\mathrm{CO}_{2}$, zone airflow, daylight levels, occupancy levels, etc.), which are, in turn, integrated through an EMCS and an array of electronic actuators for variable air volume (VAV) boxes, terminal unit controllers to process sensor outputs, and control airflow (CABA 2002). ${ }^{1}$ However, many of these features have achieved negligible market penetration. For instances, the global market for IAQ sensors (including $\mathrm{CO}_{2}$ ) did not exceed ten million dollars in 2001 (BCS Partners 2002).

\subsubsection{Prevalence of Centralized Building Controls}

The Commercial Buildings Energy Consumption Survey (CBECS) estimated that the installed base of buildings with an EMCS increased markedly from about 250,000 in 1995 to 450,000 in 1999. The probability of having an EMCS increases dramatically as the building's floorspace increases (Figure 2.2). As a result, even though only about $10 \%$ of 4,650,000 commercial buildings have an EMCS, EMCSs serve about $33 \%$ of the approximately 67 billion $\mathrm{ft}^{2}$ of commercial floorspace (CBECS 1999).

According to CBECS, 450,000 (10\%) of commercial buildings had an EMCS in 1999; a dramatic increase from approximately 250,000 installations in 1995 (CBECS 1999). Four possible reasons exist for the $80 \%$ increase in EMCS installations. First, a general increase in the use of computers, accelerated by the rise of the Internet, likely led to greater computerization of building functions. Second, the functionality of building controls expanded and the user friendliness of EMCSs improved over this period. Third, prices generally decreased, increasing the attractiveness of EMCSs (BCS Partners 2002). Finally, energy service companies (ESCOs) often installed EMCSs in buildings as an energy-saving measure for performance contracting.

\footnotetext{
${ }^{1}$ CABA (2002) describes several “intelligent” building deployments and technologies.
} 


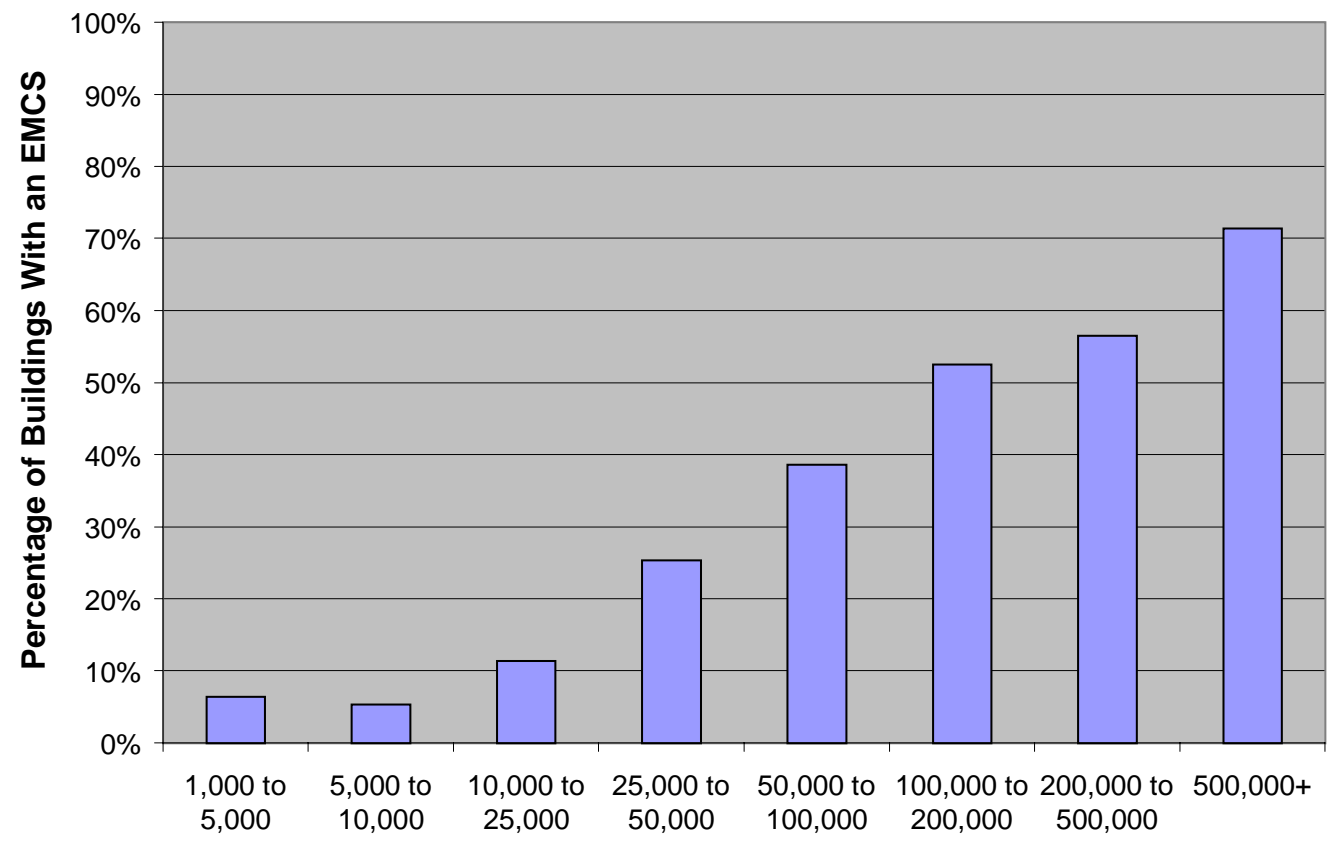

Building Size Range, Sq. Ft.

Figure 2.2. Percentage of Buildings with an EMCS, by Building Size Range (from CBECS 1999)

The first cost of an EMCS inhibits their deployment in smaller buildings. In addition, smaller buildings tend to have fewer zones, require less sophisticated controls than larger buildings, and may not reap the same energy and maintenance benefits from the centralized control. Instead, most buildings without an EMCS have very basic building controls, i.e., thermostats (with setback capability) to control air temperature in the different building zone(s). Recently, major building controls vendors have begun offering products specifically targeted at light commercial buildings that offer some EMCS-like functionality, such as remote access, multi-zone control, system monitoring, diagnostics, scheduling and setback, alarming, demand control, data logging and archiving, etc. Many of these products are designed for integration with and control of one or more packaged rooftop units, which are prevalent in light commercial buildings.

Figure 2.3 shows that EMCSs have achieved the greatest market penetration in education and office buildings. ${ }^{1}$ The 1995 CBECS data also suggest that occupancy sensors served some portion of about 6 billion $\mathrm{ft}^{2}$ of floorspace - at most $10 \%$ of all commercial building floorspace. ${ }^{2}$ Presumably, most were integrated with lighting controls.

\footnotetext{
${ }^{1}$ Included in the health care data are hospitals, which also have a higher-than-average percentage of floorspace and buildings served by an EMCS.

${ }^{2}$ BOMA (2000) found a similar market penetration in their survey of commercial building owners. Occupancy sensors integrated with lighting controls have much higher market share in California; see RLW (1999) for additional information about occupancy and daylight sensors applied to lighting.
} 


\subsubsection{Market Size}

Table 2.5 summarizes the sales of building controls in the commercial buildings sector in 2001. In this context, BCS Partners 2002 defines the term "building control systems" as "proprietary control systems platforms, related equipment, and proprietary software," including only DDC systems. Table 2.5 reveals the following:

- Maintenance and spare part expenditures are much larger than purchases of building control systems and instruments and actuators, indicating the market importance of maintaining existing building controls

- System installation, including wiring and electrical work, account for more than half of the installation budget

- Operator training accounts for a rather small - but not insignificant - portion of building control system expenses.

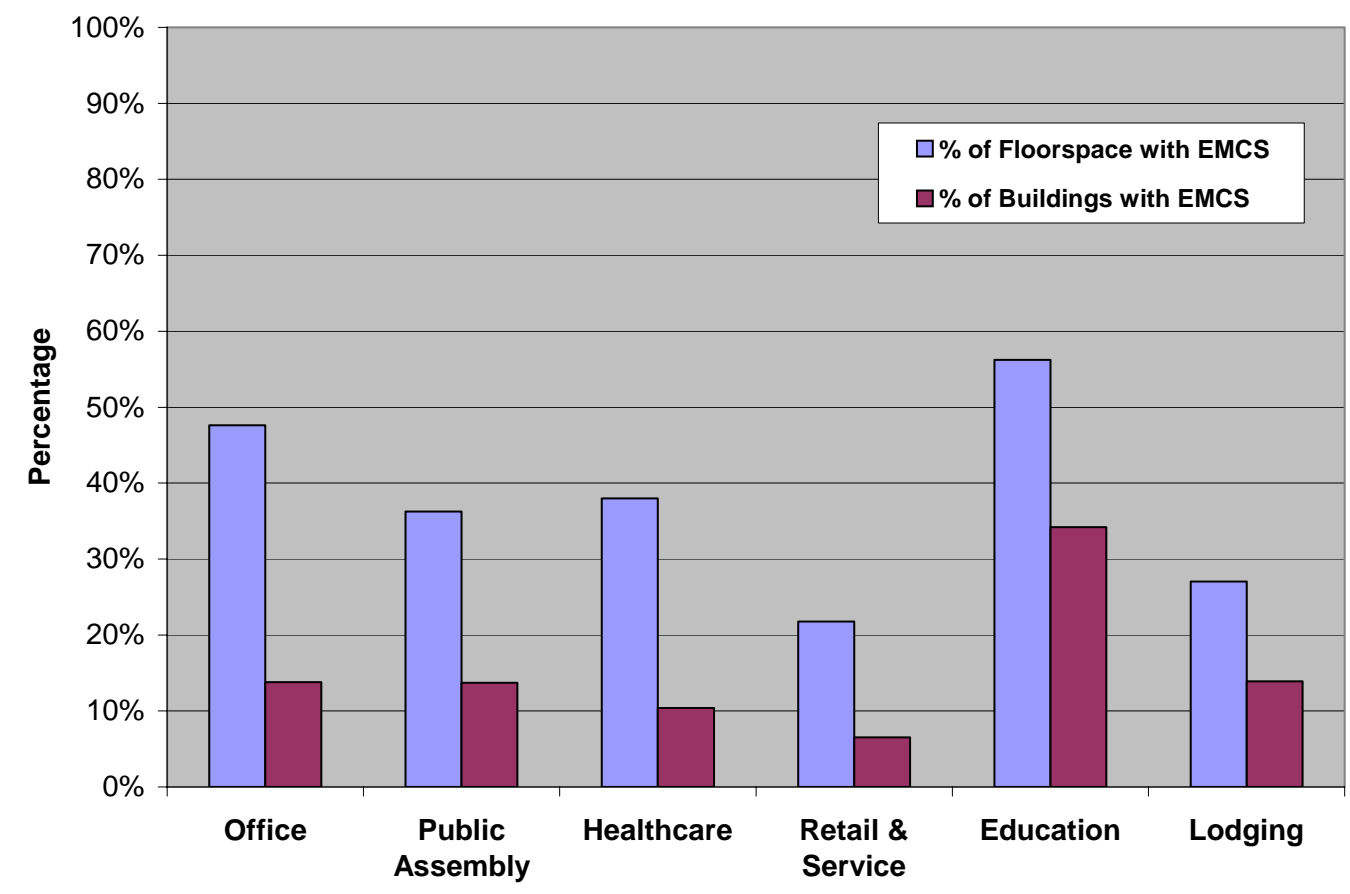

Figure 2.3. Prevalence of EMCSs by Building Type, by Percentage of Floorspace and Total Buildings (CBECS 1999)

In comparison, other building systems in commercial buildings, including fire protection and security and elevator monitoring, have a combined annual sales of about $\$ 2$ billion. U.S. sales of dedicated lighting control systems and sensors totaled about $\$ 80$ million in 2001, with occupancy sensors probably accounting for at least half of this total. Remote monitoring services totaled approximately $\$ 75$ million (BCS Partners 2002).

Moderate growth of about $2 \%$ per year in dollar terms is projected for most building control system products over the next several years. An exception is network devices, which project higher growth of 
approximately 5\% per year (compound annual growth rate). However, significant growth in unit sales will likely occur as the cost per device continues to decrease for all DDC equipment (BCS Partners 2002).

Office and commercial buildings (primarily mercantile/retail) account for about half of building control system annual expenditures. Office, industrial (conditioned space), and, to a lesser degree, educational buildings have a higher investment in control expenditures per $\mathrm{ft}^{2}$ (BCS Partners 2002). Existing buildings account for about $75 \%$ to $80 \%$ of new building control system installations and expenditures at present and this trend will likely continue for the next few years (BCS Partners 2002). In the new construction market, EMCS installations closely track the volume of new construction; the education, government, and healthcare sectors represent the largest market segments.

Table 2.5. Annual U.S. Sales of Building Controls Equipment and Services (based on BCS Partners 2002)

\begin{tabular}{|c|c|}
\hline Category & $\begin{array}{l}\text { Approximate U.S. } \\
\text { Sales - 2001 } \\
\text { [millions \$US }^{\text {(a) }}\end{array}$ \\
\hline Building Control Systems & $\$ 340$ \\
\hline Terminal Controllers ${ }^{(\mathrm{b})}$ & $\$ 110$ \\
\hline System Controllers ${ }^{(c)}$ & $\$ 145$ \\
\hline Network Devices ${ }^{(\mathrm{d})}$ & $\$ 80$ \\
\hline Instruments and Actuators & $\$ 400$ \\
\hline Building Control System Installation $^{(\mathrm{e})}$ & $\$ 930$ \\
\hline Application Engineering (Hardware configuration, schematics, software) & $\$ 240$ \\
\hline System Installation, Wiring, Electrical & $\$ 525$ \\
\hline System Start-Up & $\$ 90$ \\
\hline Operator Training & $\$ 75$ \\
\hline Building Control System Maintenance \& Spare Parts & $\$ 1,175$ \\
\hline Other & $\$ 70$ \\
\hline TOTAL & $\$ 3,100$ \\
\hline $\begin{array}{l}\text { (a) Note: Imperfect sums reflect rounding. } \\
\text { (b) Unitary DDC controllers for zone, vent, VAV, etc. } \\
\text { (c) Rooftop, AHU, chiller, EMS, other multi-loop controllers } \\
\text { (d) Central workstations, application software (from BCS vendor), comm } \\
\text { (e) Includes commissioning. }\end{array}$ & tions hardware, etc. \\
\hline
\end{tabular}

\subsubsection{Communications}

Communications play a major role in enabling building-wide controls. Communication protocols dictate communication between devices and are central to the question of interoperability, that is, whether or not devices can share essential information to allow effective control function. Communications protocols denote the physical media through which control information and commands pass between devices (e.g., twisted-pair wiring) and have a substantial impact on the installed cost of building controls. Several advances have occurred in both areas over the prior two decades, particularly in protocols, with major ramifications for the functionality and cost of building control systems. The advent of direct digital control (DDC) markedly increased the ease of information feedback and exchange between points, allowing a much greater range of potential control strategies and providing superior reliability. 
Initially, almost all DDC systems relied upon proprietary communications protocols. In the 1990s, customers began to demand "open" communication protocols that would allow them to consider and select equipment, sensors, and control software with the most attractive features for each building. In this environment, two open communications protocols came to market, BACNet ${ }^{\mathrm{TM}}$ and LonTalk. ${ }^{\circledR 1}$ Although each protocol can be used to realize interoperability, they are not interoperable with each other.

Kranz and Gisler (2002) note that many more manufacturers produce LonTalk ${ }^{\circledR}$-based devices for building applications, which should provide a broader range of potential functionality as well as more competitive device pricing. On the other hand, they believe BACNet ${ }^{\mathrm{TM}}$ represents the best option for EMCS control because it offers greater top-level functionality and interoperability with enterprise networks (i.e., with Ethernet and IP). According to long-time industry analysts, reliable information about the relative market shares of different communications protocols is not available (BCS Partners 2002).

In spite of efforts to develop interoperable systems based on open protocols, this goal generally remains elusive. A recent "Technology Roadmap for Intelligent Buildings" notes that "currently, adherence to standards and protocols that ensure interoperability among diverse systems does not generally exist in the marketplace for intelligent building technologies" (CABA 2002). ${ }^{2}$ Fire and life safety systems are more likely to have proprietary controls due to their critical nature. However, interoperability and integrated communications can be achieved by a single vendor or via "middleware” (CABA 2002).

The market has begun to exploit enterprise networks to communicate information, allowing building owners to reduce the installed cost of building controls by sharing communications infrastructure. For example, a building operator can remotely access data and control a building from any device with Internet access (PC, hand-held device, cell phone) and appropriate access permission. In the future, this trend could devolve much control of occupied space to building occupants by allowing input on space conditions (temperature, light levels, etc.) over existing enterprise networks.

Since the advent of DDC, twisted pair wiring has become the standard medium for communication. Typically, each building system (HVAC, fire, security, building access, vertical transport, communications, etc.) is installed by a different entity at a separate point in time, with independent wiring/conduits and separate communications terminals for each system. In the context of integrated building systems, forward-looking parties have begun considering the possibility of sharing a single wiring installation.

In existing buildings, the cost of installing additional cabling to communicate with new sensors or to integrate existing sensors into an EMCS can be very high. This cost is, not infrequently, prohibitive, due to the complexity of pulling and snaking wires through the existing structure. A variety of wireless communications approaches offer the promise of lower-cost installation for retrofit applications, most notably in applications requiring longer cable runs and/or having problematic access, and requiring infrequent communication due to limited power availability (Kintner-Meyer et al. 2002). As wireless communications electronics become more power-efficient and their cost decreases further, they have the

\footnotetext{
${ }^{1}$ EIB/KONNEX is third protocol for interoperability, deployed primarily in Europe (Kranz and Gisler 2002).

${ }^{2}$ Kranz and Gisler (2002) note the same issue.
} 
potential to have a significant impact on the building controls and systems communications infrastructure of the future.

On a very limited scale, wireless communications have also been used to perform hands-off monitoring and diagnostics of equipment. For example, packaged rooftop units, instrumented with temperature and pressure sensors and a wireless transmitter have been used to check efficiency and diagnose problems. ${ }^{1}$

Rossi estimated that the wireless diagnostics system roughly halves the time required for typical maintenance in some applications (e.g., multi-unit rooftops serving a larger store).

\subsection{Market-Achievable Energy-Savings Potential}

Building controls have the potential to realize significant energy savings in commercial buildings.

Overall, the approximately 4,700,000 commercial buildings in the United States (with a total floor space of over 67-billion $\mathrm{ft}^{2}$ ) consume about 17.5 quadrillion Btus (quads) of primary energy per year, or about $18 \%$ of all the energy consumed in the United States (see Figure 2.4$)^{2}$. Energy consumption associated with functions addressed by conventional building controls, i.e., lighting, heating, cooling, and ventilation, totals nearly 10 quads, or $57 \%$ of primary energy. These 10 quads broadly frame the energy savings opportunity for building controls.

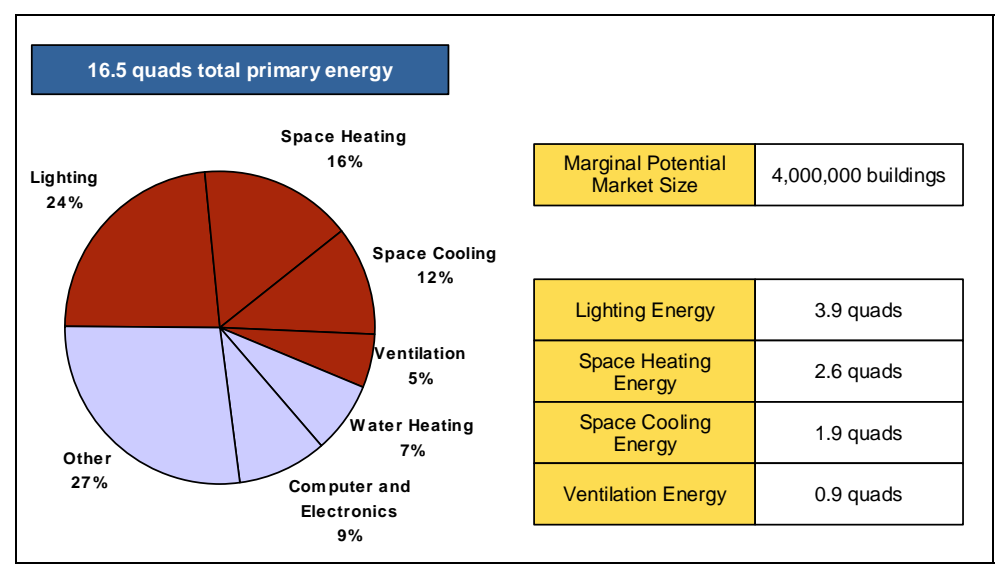

Figure 2.4. Market Size and Energy Consumption of Commercial Buildings (BTS 2002)

Of the total floor space, only a portion is heated, cooled, and lit. Consequently, the marginal potential market size (total remaining available) for a building control approach that addresses lighting would be equal to the lit commercial building floorspace (approximately 55 billion $\mathrm{ft}^{2}$; CBECS 1999), less the quantity of commercial floorspace that is already served by the control approach under consideration. ${ }^{3}$ The energy that would be saved if a given control approach captured the marginal potential market size equals the technical energy savings potential.

\footnotetext{
${ }^{1}$ Personal communication with Rossi, TM, Field Diagnostic Services.

${ }^{2}$ Buildings Core Databook, 2004, Table 1.3.3.

${ }^{3}$ The units used to evaluate penetration vary with approach, for example, the number of buildings commissioned or the percentage of floorspace served by occupancy sensors.
} 
In reality, each controls approach can only capture a portion of the marginal potential market size due to economic factors such as the simple payback period (SPP) ${ }^{1}$. In addition, non-economic factors also determine the market-achievable energy savings potential of an approach. Thus, the "remaining market penetration” estimates should be viewed as providing guidance on basic trends rather than being quantitatively precise. It is important to note that all the values calculated reflect the current assessment of each technology. Future advances that alter the efficacy and/or cost of an approach could significantly change the market-achievable values assessment. The Appendix presents details of the energy savings and SPP calculations.

The technical - and particularly the market-achievable - energy savings potential estimates developed for the control approaches examined reflect information garnered from multiple case studies. As such, there is significant uncertainty associated with extrapolating energy savings potential and cost impact to the national population of buildings. For example, energy savings generally are calculated relative to the baseline building energy use, and therefore reflect the prior state of that specific building as well as the efficacy of the measure. Clearly, a poorly operated building will benefit more from diagnostics than a well-run building. Furthermore, many case studies include other measures besides building controls, e.g., lighting retrofits, adding uncertainty to the estimated energy savings associated the building control measure. Finally, many measures (e.g., commissioning) may be implemented in - and also reported on only in cases where they realize the greatest operating cost and energy savings benefits, potentially biasing the data toward higher energy savings.

Other potential confounding issues include geography (weather, building practice) and local utility rates (including rate structures). Consequently, the energy savings and payback period estimates need to be viewed as general estimates of the approximate magnitude of the potential opportunity of each approach. In some instances, major data gaps exist that impede estimation of a credible estimate. These gaps are noted and are also reflected in the very broad market penetration and energy saving potential ranges estimated for some technologies.

Table 2.6 summarizes the market-achievable energy savings estimates for several building control approaches. The energy savings from different measures are not necessarily additive. Furthermore, this is not a comprehensive list of possible controls-based energy savings measures but, instead, provides a general feel for the magnitude of energy savings possible focusing on a limited number of approaches that have received prior study. Estimates of the approximate market size (in terms of floor space) and marketachievable national energy savings of the control approaches are presented.

\footnotetext{
${ }^{1}$ The simple payback period equals the incremental installed cost of a measure divided by the net annual savings (primarily from energy savings) achieved by that measure. Unlike a net-present value calculation (NPV), it does not take into account the time value of money, i.e., no discount rate.
} 
Table 2.6. Summary of Energy Savings Potential for Control Approaches

\begin{tabular}{|l|c|c|c|c|c|c|c|}
\hline Control Technology & $\begin{array}{c}\text { Technical } \\
\text { Market Size } \\
\text { [billions ft }{ }^{2} \text { ] }\end{array}$ & $\begin{array}{c}\text { Relevant } \\
\text { Primary } \\
\text { Energy } \\
\text { [quads] }\end{array}$ & $\begin{array}{c}\text { Energy } \\
\text { Savings } \\
\text { [\%] }\end{array}$ & $\begin{array}{c}\text { Technical } \\
\text { Energy } \\
\text { Savings } \\
\text { Potential } \\
\text { [quads] }\end{array}$ & $\begin{array}{c}\text { Simple } \\
\text { Payback } \\
\text { Period } \\
\text { [years] }\end{array}$ & $\begin{array}{c}\text { Remaining } \\
\text { Market } \\
\text { Penetration }\end{array}$ & $\begin{array}{c}\text { Market- } \\
\text { Achievable } \\
\text { Energy } \\
\text { Savings } \\
\text { [quads] }\end{array}$ \\
\hline $\begin{array}{l}\text { Energy Management } \\
\text { and Control System } \\
\text { (EMCS) }\end{array}$ & 33 & 6.2 & $5-15 \%$ & $0.3-0.9$ & $8-10$ & $5-10 \%$ & $0.02-0.09$ \\
\hline Commissioning & 55 & $9.8^{(\mathrm{a})}$ & $5-15 \%$ & $0.5-1.5$ & $2-10$ & $3-30 \%$ & $0.015-0.5$ \\
\hline $\begin{array}{l}\text { Automatic Fault } \\
\text { Detection and } \\
\text { Diagnostics } \\
\text { (AFDD)/Continuous } \\
\text { Commissioning }\end{array}$ & 55 & $9.8^{(\mathrm{a})}$ & $5-15 \%$ & $0.5-1.5$ & $1-3$ & $\begin{array}{c}15-55 \% / \\
6-24 \%\end{array}$ & $0.07-0.8 /$ \\
\hline $\begin{array}{l}\text { Occupancy Sensors for } \\
\text { Lighting Control }\end{array}$ & 50 & 3.5 & $20-28 \%$ & $0.7-1.0$ & $1-5$ & $0-45 \%$ & $0-0.35$ \\
\hline $\begin{array}{l}\text { Photosensor-Based } \\
\text { Lighting Control }\end{array}$ & 55 & 3.9 & $20-60 \%$ & $0.8-2.3$ & $1-7$ & $8-55 \%$ & $0.08-1.3$ \\
\hline $\begin{array}{l}\text { Demand Controlled } \\
\text { Ventilation (DCV) }\end{array}$ & 55 & 5.4 & $10-15 \%$ & $0.5-0.8$ & $2-3$ & $15-30 \%$ & $0.08-0.25$ \\
\hline
\end{tabular}

(a) This figure includes 0.5 quads for supermarket refrigeration systems and walk-in refrigeration.

\subsubsection{Energy Management and Control Systems (EMCS)}

\subsubsection{Definition}

An Energy Management and Control System (EMCS) is a centralized system that receives and monitors information from various sensors deployed in the building. It allows the building owner to effect control actions based on the sensors' outputs. EMCSs may be very simple and limited (perhaps only performing system monitoring and data visualization) or they may integrate all building systems and include automated control. An EMCS also enables automation of various physical tasks that would otherwise be performed manually at a specific piece of equipment (such as operating dampers daily to keep them from sticking). The actual equipment in an EMCS system consists of a central or distributed computing device, communications wires or pathways, sensors, actuators, and a software suite that may include complex logic and visualization functionality.

\subsubsection{National Energy Savings}

An authoritative study on the nationwide impact of EMCSs on energy consumption does not yet exist. In fact, much confusion remains over the definition of EMCSs and how to isolate the energy savings that are directly attributable to EMCSs from other system benefits that could be achieved without EMCSs (such as programmable thermostats and set-back clocks). Further confusion surrounds how much energy an EMCS can save due to the very broad range of functionality. Studies have demonstrated that EMCSs can provide data visualization and monitoring services to help building operators find and correct energywasting malfunctions (Taylor and Pratt 1989; Piette et al. 1998, 2000, 2001, 2003; Taylor 1992; and Claridge 1998). Further complicating the issue are findings that many EMCSs do not function properly 
when installed or do not achieve the energy savings predicted (Energy Design Resources 1998; Roth et al. 2002). Studies performed over the past decade reveal that some EMCS installations attain negligible energy savings while others realize annual savings of over 30\% (Wortman et al. 1996; Guillemen and Morel 2001; Wheeler 1994).

Roughly gauging the range of energy savings from the literature, EMCSs typically appear to achieve energy savings between $5 \%$ and $15 \%$. This range accounts for the difference in building types, system types, and other variables. Undoubtedly, some cases show much higher or lower energy savings, but they often represent isolated cases with confounding factors (such as an EMCS upgrade that also included the repair of malfunctioning equipment). Other summary discussions in the literature also use the 5\% to $15 \%$ range, but tend to suggest that nationwide energy savings are on the low end of the range in part because many EMCSs do not function as intended (Energy Design Resources 1998; ADL 1999; Roth et al. 2002).

Table 2.7. Summary of Energy Management and Control System (EMCS) Energy Savings

\begin{tabular}{|c|c|c|}
\hline Attribute & Value & Notes \\
\hline Technical Market Size & 33 billion $\mathrm{ft}^{2}$ & Total floor space, less 33\% served by an EMCS \\
\hline $\begin{array}{l}\text { Relevant Annual Energy } \\
\text { Consumption }\end{array}$ & 6.2 quads & 2/3rds of heating, cooling, lighting, and ventilation \\
\hline Energy Savings & $5 \%-15 \%$ & $\begin{array}{l}\text { A nationwide average may tend toward the low end of the } \\
\text { range because EMCSs often does not perform as well as } \\
\text { intended over their lifetime. }\end{array}$ \\
\hline $\begin{array}{l}\text { Technical Annual Energy } \\
\text { Savings Potential }\end{array}$ & $0.3-0.9$ quads & \\
\hline $\begin{array}{l}\text { Commercial Building Peak } \\
\text { Reduction }\end{array}$ & $5 \%-10 \%$ & \\
\hline Simple Payback Period & $8-10$ years & $\begin{array}{l}\text { Some regional studies claim shorter paybacks, but the data } \\
\text { point toward higher paybacks for a national average. }\end{array}$ \\
\hline $\begin{array}{l}\text { Ultimate Additional } \\
\text { Market Penetration }\end{array}$ & $5 \%-10 \%$ & $\begin{array}{l}\text { Non-energy cost drivers likely play a significant role, i.e., } \\
\text { current market share exceeds the } 3 \%-5 \% \text { market share } \\
\text { suggested by an } 8-10 \text { year payback. }\end{array}$ \\
\hline Market Potential & $\begin{array}{r}1.7-3.3 \\
\text { billion } \mathrm{ft}^{2}\end{array}$ & \\
\hline $\begin{array}{l}\text { Market-Achievable Annual } \\
\text { Energy Savings }\end{array}$ & $\begin{array}{l}0.02-0.09 \\
\text { quads }\end{array}$ & \\
\hline
\end{tabular}

\subsubsection{Percent Peak Reduction}

EMCSs allow strategies for peak-shaving, such as air-conditioning load deferment and demand limiting, which could notably reduce the peak electric load in any given month during the summer. Few casespecific data are available on peak load reductions of EMCSs, though Wortman et al. (1996) did show summer peak load reductions in the range of $5 \%$ to $9 \%$. An independent, though very rough calculation shows that if the air-conditioning load accounts for roughly $50 \%$ of electric loads during peak hours (Nadel 2000) and EMCSs can shave that load by approximately 20\%, then the EMCS reduces peak demand by approximately $10 \%$. 


\subsubsection{Simple Payback Period}

A general lack of comprehensive information on nationwide EMCS costs and payback periods exists, though some payback information exists for specific case studies. Unfortunately, information gleaned from case studies tends to be installation-specific due to energy costs and utility rate structures, and may also include non-EMCS cost and/or energy savings. Various regional case studies claim between 1- and 10-year payback periods (Buildings.com 2003, Piette et al. 2000, and Hill et al. 2000). On a cost-per- $\mathrm{ft}^{2}$ basis, EMCSs have a very broad range, from approximately \$1-\$4 per square foot (Piette et al. 2000; Energy Design Resources 1998) suggesting payback periods closer to 10 years at the national scale.

Other discussions also suggest that payback periods are between 8 and 10 years (ADL 1997). While more information about EMCS's installed costs and corresponding annual energy savings are needed in order to apply nationwide average energy costs for a defensible payback calculation, EMCSs seem to have payback periods of approximately 8 to 10 years based on energy cost savings alone. Reduced maintenance and operations costs - the original reason for deployment of EMCSs - will tend to decrease payback periods. However, as Barsoum (1995) notes, "the benefits derived from up-front investments are usually too vague to measure, thus monies spent are usually only justified versus energy dollars 'avoided'." Hence, although an EMCS does reduce O\&M costs, they tend not to factor in the economic assessment. Payback periods will tend to be longer for smaller buildings because the installed cost of the EMCS does not scale linearly with the magnitude of energy cost savings.

\subsubsection{Ultimate Market Penetration}

Based on the market penetration curve (see the Appendix), an eight- to ten-year payback yields a 3\% to 5\% ultimate market penetration. EMCSs have been on the market for about 20 years and have already penetrated 33\% of all commercial building floor space, but only $10 \%$ of all buildings (CBECS 1999). If market size is based on floor space, then EMCSs have already exceeded their ultimate market potential (based only on energy-related payback). This indicates that non-energy factors drive EMCS market penetration, making it difficult to estimate how much of the market remains to be penetrated.

The dramatic increase in the installed base of EMCSs in the late 1990s, which occurred in spite of low energy prices, provides further evidence for the de-coupling of EMCS market penetration from energybased economics. Based on limited information, the remaining market penetration is estimated to range from 5\% to 10\%. Given the increase in the number of buildings with an EMCS from around 250,000 in 1995 to about 450,000 in 1999, this estimate may be conservative.

\subsubsection{Commissioning}

\subsubsection{Definition}

Commissioning is a service provided by specialized contractors. To commission an existing building, the contractor inspects and evaluates the performance of the building and its systems to detect and diagnose substandard and malfunctioning equipment and sensors (e.g., inefficient setpoints and poorly tuned control feedback settings). The contractor may also recommend possible energy-savings upgrades. After diagnosing the problems, the contractor or another party fixes the problems to reduce building energy consumption. 


\subsubsection{National Energy Savings}

A significant volume of literature suggests that ill-functioning building systems and equipment waste a significant quantity of energy, in some cases equal to up to $30 \%$ of the energy consumed by commercial buildings (Ardehali et al. 2003; Ardehali and Smith 2002; Claridge et al. 1994, 1996, and 2000). Various studies show that the annual energy savings achieved by commissioning range consistently between $5 \%$ and 15\%. Parks and Kellow (2000), for instance, showed field test results for four buildings that showed annual energy savings of $2 \%$ to $13 \%$ from commissioning existing buildings. Claridge et al. (1994 and 1996) reported savings of around $10 \%$ on average by commissioning existing buildings. Kumar (2001) noted that "the potential savings out of improved energy management and faulty and non-optimal operation of HVAC systems alone in commercial buildings is estimated to be $20 \%-30 \%$," but this is higher than most existing information can support.

The actual energy savings for a given building vary greatly and depend on the types of systems in a building, how well building operators maintain the building, and what failures occur (e.g., if a failure does not impact comfort, such as an economizer, it may escape detection). In practice, however, the energy savings initially gained from commissioning do not always persist because building problems arise over time due to equipment aging, changes in building usage, alterations in control settings, etc. Consequently, most buildings require follow-up commissioning at regular intervals to maintain high performance levels.

Unfortunately, very little information, particularly quantitative information, exists about the persistence of commissioning energy savings, making development of a national estimate of persistence impossible. (Potter et al. 2002) studied ten buildings that were commissioned at least two years prior to their study and found that over half of the commissioning "fixes" persisted. Hardware modifications showed a greater propensity to persist but savings from control strategies often did not persist, presumably due to changes made by operators (e.g., setpoints, scheduling). This shows general consistency with Turner et al. (2001), whose study seems to indicate that many buildings and building systems maintain energy savings while others see energy savings degrade by several percent a year. However, insufficient data currently exist to develop a credible estimate of the persistence of energy savings from commissioning.

Since the initial savings from commissioning appear to range between $5 \%$ and $15 \%$, this represents an upper bound for the energy savings from commissioning, i.e., assuming no degradation over time. At present, a lower bound cannot be reasonably determined. Table 2.8 summarizes the potential energy savings. 
Table 2.8. Summary of Commissioning Energy Savings

\begin{tabular}{|c|c|c|}
\hline Attribute & Value & Notes \\
\hline Technical Market Size & 55 billion $\mathrm{ft}^{2}$ & Almost all commercial floor space \\
\hline $\begin{array}{l}\text { Relevant Annual Energy } \\
\text { Consumption }\end{array}$ & 9.8 quads & $\begin{array}{l}\text { Heating, cooling, lighting, ventilation, and 50\% of } \\
\text { refrigeration energy }\end{array}$ \\
\hline Energy Savings & $5 \%-15 \%$ & $\begin{array}{l}\text { Upper bound; in practice, lower because of } \\
\text { degradation of energy savings after commissioning }\end{array}$ \\
\hline $\begin{array}{l}\text { Technical Annual Energy } \\
\text { Savings Potential }\end{array}$ & $0.5-1.5$ quads & \\
\hline $\begin{array}{l}\text { Commercial Building Peak } \\
\text { Reduction }\end{array}$ & $5 \%-15 \%$ & $\begin{array}{l}\text { Very rough estimate based on energy savings } \\
\text { potential }\end{array}$ \\
\hline Simple Payback Period & $2-10$ years & Very broad range reported in literature \\
\hline Ultimate Market Penetration & $3 \%-30 \%$ & $\begin{array}{l}\text { Non-energy benefits may cause penetration to tend to } \\
\text { approach the higher end of the range }\end{array}$ \\
\hline Market Potential & $\begin{array}{l}1.7-16.5 \\
\text { billion } \mathrm{ft}^{2}\end{array}$ & \\
\hline $\begin{array}{l}\text { Market-Achievable Annual } \\
\text { Energy Savings }\end{array}$ & $\begin{array}{l}0.015-0.5 \\
\text { quads }\end{array}$ & \\
\hline
\end{tabular}

\subsubsection{Percent Peak Reduction}

Commissioning can improve the efficiency of air-conditioning equipment and distribution systems that account for the largest portion of commercial building peak demand. Some common errors that commissioning could detect, such as economizer malfunctions (stuck open during cooling mode for example) have substantial impacts on peak load. For instance, Roth et al. (2002) estimated that economizer malfunction can waste between $20 \%$ and $30 \%$ of all HVAC energy consumed and, at any particular time, approximately half of all economizers are malfunctioning. Since HVAC systems account for approximately 50\% of commercial building peak demand (Nadel 2000) if an economizer malfunction was wasting $30 \%$ of that energy, addressing this malfunction would reduce the peak by $15 \%$. On the other hand, many problems, e.g., lights on at night, may not have any impact on peak demand. A very rough estimate for the impact of commissioning on peak demand would be that the peak demand reduction is similar to the energy savings from commissioning, i.e., on the order of $5 \%$ to $15 \%$.

\subsubsection{Simple Payback Period}

The literature reports payback periods between 2 and 10 years (Buildings.com 2003; Hill et al. 2000; Piette et al. 2001; Roth et al. 2002). These estimates typically do not take into account persistence effects. The estimates vary widely based on location and application. Additional data are needed to develop a more representative estimate of the average payback period on the national level.

\subsubsection{Ultimate Market Penetration}

Based on a two- to ten-year payback period, the "A" market penetration curve estimates an ultimate market penetration of $3 \%$ to $30 \%$ for commissioning. Current building commissioning rates are very low, less than $5 \%$ for new buildings ${ }^{1}$ and well under $1 \%$ for existing buildings (Engineered Systems 1999). In general, lack of awareness of commissioning and the cost of commissioning appear to bear responsibility

\footnotetext{
${ }^{1}$ Public buildings appear to have significantly higher commissioning rates due to mandates and/or practice (RLW 1999; Quantum 2003).
} 
for low commissioning rates (RLW Analytics 1999). Therefore, the full market remains to be penetrated. Commissioning also has notable non-energy savings benefits including improved occupant comfort and longer equipment lifetime, but the literature currently focuses on energy savings with limited work to quantify the impact of these non-energy benefits on market penetration.

\subsubsection{Automatic Fault Detection and Diagnostics (AFDD) / Continuous Commissioning}

\subsubsection{Definition}

Automated fault detection and diagnostics (AFFD) and Continuous Commissioning are similar softwarebased processes that monitor and analyze building, system (e.g., chiller), and/or equipment (e.g., unitary roof-top air conditioners) performance for the purpose of detecting and diagnosing subpar performance and faults. AFFD focuses on monitoring equipment and/or system behavior to diagnose faults, while continuous commissioning usually also includes functional testing and evaluation of building equipment and systems to detect and diagnose faults. When performed on a system or building-wide level, both AFDD and continuous commissioning usually use information provided by an EMCS, often with minimal human operator support.

\subsubsection{National Energy Savings}

The energy savings potential of AFDD/continuous commissioning is identical to that of commissioning (see above), except that the energy savings do not degrade over time because of the continuous nature of these processes. Therefore, they can realize the full 5\% to 15\% energy savings identified in the literature for commissioning (Parks and Kellow 2000; Claridge et al. 1994 and 1996; Roth et al. 2002). For AFDD, this range assumes that AFDD is applied to all major energy-consuming building systems and equipment. In the case of equipment-level AFDD, the energy savings have much greater uncertainty, as the probability of common faults and the distribution of their energy impact for major equipment types (packaged rooftop units, chillers, furnaces, etc.) are not well understood. On average, equipment-level AFDD appears to have an energy savings potential roughly similar to that of commissioning; naturally, the savings only apply to the equipment monitored by AFDD. Table 2.9 presents an overview of the potential energy savings for AFDD and continuous commissioning.

\subsubsection{Peak Demand Reduction}

Building-wide AFDD/continuous commissioning should achieve peak demand reductions similar to those of continual commissioning, i.e., roughly 5\% to 15\%. In contrast, equipment-specific AFDD depends on the piece of equipment monitored and the impact that piece of equipment has on peak demand. For example, AFDD for the chiller of a packaged rooftop AC unit can detect problems that have a major impact on peak demand reductions, whereas boiler AFDD will have little (if any) effect on peak electric demand. As noted above, the equipment-specific peak demand impact has much greater uncertainty, as the probability of common faults and the distribution of their energy impact for major equipment types (packaged rooftop units, chillers, furnaces, etc.) are not well understood. 
Table 2.9. Summary of AFDD/Continuous Commissioning Energy Savings

\begin{tabular}{|l|c|l|}
\hline Attribute & Value & Notes \\
\hline Technical Market Size & 55 billion $\mathrm{ft}^{2}$ & Almost all commercial floor space \\
\hline $\begin{array}{l}\text { Relevant Annual Energy } \\
\text { Consumption }\end{array}$ & 9.8 quads & $\begin{array}{l}\text { Heating, cooling, lighting, ventilation, and } 50 \% \text { of } \\
\text { refrigeration energy }\end{array}$ \\
\hline Energy Savings & $5 \%-15 \%$ & Similar to initial savings from commissioning \\
\hline $\begin{array}{l}\text { Technical Annual Energy } \\
\text { Savings Potential }\end{array}$ & $0.5-1.5$ quads & \\
\hline $\begin{array}{l}\text { Commercial Building Peak } \\
\text { Reduction }\end{array}$ & $5-15 \%$ & Similar to initial savings from commissioning \\
\hline Simple Payback Period & $1-3$ years & $\begin{array}{l}\text { Very approximate; may be longer due to cost of } \\
\text { implementing hardware fixes }\end{array}$ \\
\hline Ultimate Market Penetration & $\begin{array}{c}15-55 \% / \\
6-24 \%\end{array}$ & $\begin{array}{l}\text { Lower values for continuous commissioning reflects } \\
\text { limited market of buildings with EMCSs }\end{array}$ \\
\hline Market Potential & $\begin{array}{c}8-30 / 3-13 \\
\text { billion ft }\end{array}$ & \\
\hline $\begin{array}{l}\text { Market-Achievable Annual } \\
\text { Energy Savings }\end{array}$ & $\begin{array}{c}0.07-0.8 / 0.03- \\
0.35 \text { quads }\end{array}$ & Stand-alone and EMCS-based systems, respectively \\
\hline
\end{tabular}

\subsubsection{Simple Payback Period}

Case studies suggest that AFDD will pay for itself in one to three years in larger buildings (Piette 2001; Claridge et al. 1999; Roth et al. 2002), with the actual simple payback period (SPP) depending upon the specific application (types of measures, AFDD sophistication, building type, building size, etc.) and the energy cost structure. Since AFDD works with a building's EMCS, much of the hardware needed for data monitoring already exists. The cost of the AFDD system is limited to the software cost, labor costs, and limited additional hardware costs. It is reasonable, therefore, that AFDD will payback more quickly (one to three years) than an EMCS alone (eight to ten years), or than commissioning (which requires extensive hands-on effort). However, these data do not appear to take into account the cost of fixing the diagnosed system or equipment problems, which could increase the SPP. For sub-optimal control issues, e.g., poorly tuned controller or inappropriate setpoints, AFDD can potentially remedy these problems automatically and at little incremental cost, but hardware fixes will require additional expenditures.

\subsubsection{Ultimate Market Penetration}

Based on a one- to three-year payback, the "A" market penetration curve suggests an ultimate market penetration of $15 \%$ to $55 \%$. Since building-level AFDD/continuous commissioning requires an EMCS, one could argue that the applicable market size only includes the portion of the market that will have EMCSs. On the other hand, AFDD for individual pieces of equipment (e.g., a rooftop air-conditioner) would not require a centralized EMCS and can prove attractive for energy-intensive equipment. Thus, the ultimate market penetration estimates for continuous commissioning, $6 \%$ to $24 \%$, reflect only floorspace with an EMCS, while AFDD considers all commercial floorspace, i.e., 15\% to 55\%. In assessing the ultimate market penetration, one must also consider that the manual approach of conventional commissioning (see prior subsection) may compete with AFDD/continual commissioning. 


\subsubsection{Occupancy Sensors for Lighting Control}

\subsubsection{Definition}

Occupancy sensors for lighting control have been around for over 20 years and serve some portion (approximately 10\%) of commercial building floorspace (CBECS 1999). The most common occupancy sensor types are infra-red and ultrasonic, and they typically include a timer to compensate for when people are in a room but not moving (ultrasonic) or have entered a "dead zone" (infra-red). These sensors can either be stand-alone or integrated with an EMCS. In some cases, they can also be used to modify HVAC temperature set points, e.g., for hotel or conference rooms.

\subsubsection{National Energy Savings}

Until recently, there have been few comprehensive studies to quantify the potential energy and cost savings of occupancy sensors for lighting control. Recent investigations suggest that the energy savings are substantial, as commonly believed, but depend highly on the type of building, space occupancy patterns, and sensor placement. Market potential and energy savings potential are listed in Table 2.10.

Claims of occupancy sensor energy savings between $20 \%$ and $70 \%$ of lighting energy are common for individual spaces (Energy Design Resources 2000; VonNeida et al. 2000; Jennings et al. 2000; EIA 2001). The range of savings depends on the occupancy rate and duration, type of sensors/controls, timeout settings and adequacy of installations. Spaces that are occupied sporadically throughout the day have higher energy savings potential, such as private offices, classrooms, auditoriums, restrooms, and conference rooms (Energy Design Resources 2000).

Table 2.10. Summary of Occupancy Sensors for Lighting Control Energy Savings

\begin{tabular}{|l|c|l|}
\hline Attribute & Value & Notes \\
\hline Technical Market Size & $\sim 50$ billion $\mathrm{ft}^{2}$ & $\begin{array}{l}\text { All commercial floor space less the } 10 \% \text { served by } \\
\text { occupancy sensors }\end{array}$ \\
\hline $\begin{array}{l}\text { Relevant Annual Energy } \\
\text { Consumption }\end{array}$ & 3.5 quads & $\begin{array}{l}\text { Lighting energy (less the }<10 \% \text { of floorspace currently } \\
\text { served) }\end{array}$ \\
\hline Energy Savings & $20 \%-28 \%$ & $\begin{array}{l}\text { Larger variations for different applications, depending on } \\
\text { occupancy patterns. }\end{array}$ \\
\hline $\begin{array}{l}\text { Technical Annual Energy } \\
\text { Savings Potential }\end{array}$ & $0.7-1.0$ quads & $6 \%-8 \%$ \\
\hline $\begin{array}{l}\text { Commercial Building Peak } \\
\text { Reduction }\end{array}$ & $1-5$ years & $\begin{array}{l}\text { Wide range due to variations in physical space/layout and } \\
\text { lighting watts controlled by occupancy sensor }\end{array}$ \\
\hline $\begin{array}{l}\text { Simple Payback Period } \\
\text { Ultimate Market Penetration }\end{array}$ & $0 \%-45 \%$ & $\begin{array}{l}\text { Excludes } 10 \% \text { already penetrated (10\% - 55\% total market } \\
\text { size }\end{array}$ \\
\hline $\begin{array}{l}\text { Market Potential } \\
\text { Market-Achievable Annual } \\
\text { Energy Savings }\end{array}$ & $0-0.45$ quads \\
\hline
\end{tabular}

Public spaces with almost continuous occupancy have little potential for saving energy, such as common hallways, lobbies, or open-plan office spaces. The national lighting energy savings potential of occupancy sensors will be lower than the $20 \%$ to $70 \%$ range for individual spaces because buildings have a combination of spaces with different occupancy rates, i.e., some spaces that will not save any energy and 
others that will. In addition, some occupants actually turn out the lights when they leave their offices, in which case the savings would be zero. Without detailed breakdowns of national lighting energy consumption by type of space (hallway, private office, open-plan office), breakdown by building type gives a rough approximation of the national energy savings potential. Table 2.11 shows the breakdown of floorspace by building type and estimates for energy savings potential of each, with the net result of $20 \%$ to $28 \%{ }^{1}$ reduction in national lighting energy consumption.

Table 2.11. Occupancy Sensor Energy Savings by Building Type

\begin{tabular}{|l|l|l|l|}
\hline Building Type & $\begin{array}{l}\text { Fraction of } \\
\text { Commercial } \\
\text { Floorspace }\end{array}$ & $\begin{array}{l}\text { Approximate } \\
\text { Savings Range }\end{array}$ & $\begin{array}{l}\text { Weighted Savings } \\
\text { Range }\end{array}$ \\
\hline Education & $13 \%$ & $30 \%-40 \%$ & $4 \%-5 \%$ \\
\hline Food & $4 \%$ & $0 \%$ & $0 \%$ \\
\hline Health Care & $4 \%$ & $20 \%-30 \%$ & $\sim 1 \%$ \\
\hline Lodging & $7 \%$ & $30 \%-40 \%$ & $2 \%-3 \%$ \\
\hline Mercantile & $15 \%$ & $0 \%$ & $0 \%$ \\
\hline Office & $18 \%$ & $30 \%-40 \%$ & $5 \%-7 \%$ \\
\hline Public Assembly & $7 \%$ & $50 \%-60 \%$ & $\sim 4 \%$ \\
\hline Public safety & $2 \%$ & $0 \%$ & $0 \%$ \\
\hline Religious & $5 \%$ & $0 \%$ & $0 \%$ \\
\hline Service & $5 \%$ & $0 \%$ & $0 \%$ \\
\hline Warehouse & $16 \%$ & $25 \%-50 \%$ & $4 \%-8 \%$ \\
\hline Other & $4 \%$ & $0 \%$ & $0 \%$ \\
\hline TOTAL & $\mathbf{1 0 0} \%$ & N/A & $\mathbf{2 0 \% - 2 8 \%}$ \\
\hline
\end{tabular}

\subsubsection{Percent Peak Reduction}

Occupancy sensors are more likely to save energy during evenings and weekends, i.e., off-peak periods, than during peak periods when spaces are likely occupied. During peak electric periods, buildings are typically near full-occupancy so occupancy sensors for lighting have less impact on peak demand (VonNeida et al. 2000). But the peak reduction potential certainly exceeds zero, and studies suggest lighting load reductions of between $15 \%$ and $20 \%$ during peak periods (VonNeida et al. 2000). Since lighting energy accounts for approximately 30\% of a building's electric load (Nadel et al. 2000; Brown and Koomey 2002), the peak load impact of occupancy sensors is a reduction of approximately $4 \%$ to $6 \%$.

\subsubsection{Simple Payback Period}

There is a general lack of information on payback period and system costs for occupancy sensors for lighting control. An example case study at a large defense research campus in California showed that installing 8,000 occupancy sensors in offices, labs, and conference rooms cut lighting energy

\footnotetext{
${ }^{1}$ This value is consistent with the $25 \%$ often cited in the lighting industry (personal communications with Ron Lewis, U.S. Department of Energy, 2003).

${ }^{2}$ CBECS (1999).

${ }^{3}$ TIAX estimate based on several sources: EPA (2001), Energy Design Resources (2000), Von Neida et al. (2000), Jennings et al. (2000).
} 
consumption by 50\% giving a payback period of just over one year (Energy Design Resources 2000). Pacific Gas and Electric (2000) estimated installed costs (sensors, wiring, and commissioning) of occupancy sensors at between $\$ 0.11 / \mathrm{ft}^{2}$ and $\$ 0.56 / \mathrm{ft}^{2}$ based on interviews with contractors and using R.S. Means 2000 electrical cost data. A rough calculation of payback, assuming that annual lighting consumption is approximately $7 \mathrm{kWh} / \mathrm{ft}^{2}$ (see below ${ }^{1}$ ) and electricity costs $\$ 0.08 / \mathrm{kWh}$, shows that occupancy sensor systems will pay for themselves in about 1 to 5 years.

\subsubsection{Ultimate Market Penetration}

A one- to five-year payback period corresponds to an ultimate market penetration of approximately $10 \%$ to 55\% based on the A market penetration curve. There are few non-energy benefits that would drive higher market penetrations, and factors such as false shut-offs and reduced lamp and/or ballast lifetime might tend to limit market penetration. ${ }^{2}$ Since occupancy sensors currently serve about $10 \%$ of commercial floorspace, occupancy sensors may have achieved most of their market potential since coming to market about 20 years ago, or may have the potential for installation in an additional $45 \%$ of total commercial floorspace. ${ }^{3}$

\subsubsection{Photosensor-Based Lighting Control}

\subsubsection{Definition}

Photosensors detect the lighting level in a space and dim the artificial lighting when appropriate, either independently or with an EMCS, to automatically maintain desired lighting levels in a space. They can save energy when used to automatically dim artificial lights in response to higher space light levels arising from daylight or overlamping. In daylit spaces, photosensors measure the total amount of light in a space (both artificial and natural) and dim the artificial lights to compensate for increased natural light and maintain design lighting levels. Although photosensors for lighting control have been on the market for at least ten years (NEMA 1992), to date they have attained relatively little market penetration (PG\&E 2000).

\subsubsection{National Energy Savings}

Field tests have shown that savings from automatic daylight dimming alone can range between $10 \%$ and $60 \%$ for private office spaces, giving an average annual savings of approximately $27 \%$ (Jennings et al. 2000). A report by the EPA (2001) suggests that daylit offices can achieve up to $35 \%-40 \%$ savings, and that other daylit spaces (classrooms, grocery stores, and retail outlets) can achieve $40 \%-60 \%$ savings. Clearly, these savings apply only to perimeter or sky-lit portions of a building's floor space and the percentage of national commercial building floorspace with sufficient levels of daylight to apply automatic daylight dimming is not known.

Photosensors also can reduce lighting loads by automatically dimming lights that are over-sized for the task performed in a space or over-sized to compensate for lamp lumen depreciation (automatic tuning).

\footnotetext{
${ }^{1} 3.9$ quads (primary) divided by 55 billion $\mathrm{ft}^{2}$ of lit commercial floorspace; assumes 10,958 Btu per primary quad (BTS 2002).

${ }^{2}$ Another DOE activity, the Lighting Controls Roadmap, continues to examine this and other issues facing lighting control.

${ }^{3}$ As for all approaches discussed, this reflects their current economics.
} 
Tests suggest that many lights are oversized for the tasks being performed in a space and automatically adjusting artificial lighting levels to maintain occupant preferences can save between $20 \%$ and $60 \%$ with an annual average savings of approximately 45\% (Carter and Moore 2002; EPA 2001). Further, designers typically oversize lighting to account for lamp lumen depreciation, because the light output of a fluorescent bulb depreciates over its lifetime by $10 \%$ to $50 \%$. ${ }^{1}$ As these savings are not additive, the combined savings of automatic tuning equal roughly $20 \%$ to $60 \%$ for all buildings nationwide. Of course, these savings depend on proper commissioning to set the appropriate lighting levels for a space. In both daylight and non-daylight situations, dimming based on photosensors appears to have somewhat similar energy savings percentage ranges, i.e., 27\%-60\% and 20\%-60\%.

Table 2.12. Summary of Photosensors for Lighting Control Energy Savings

\begin{tabular}{|c|c|c|}
\hline Attributes & Value & Notes \\
\hline Technical Market Size & $\begin{array}{l}\sim 55 \text { billion } \\
\mathrm{ft}^{2}\end{array}$ & Almost all commercial building floor space \\
\hline $\begin{array}{l}\text { Relevant Annual Energy } \\
\text { Consumption }\end{array}$ & 4.4 quads & Lighting energy only \\
\hline Energy Savings & $20 \%-60 \%$ & \\
\hline $\begin{array}{l}\text { Technical Annual Energy } \\
\text { Savings Potential }\end{array}$ & $\begin{array}{l}1.0-2.3 \\
\text { quads }\end{array}$ & \\
\hline $\begin{array}{l}\text { Commercial Building Peak } \\
\text { Reduction }\end{array}$ & $10 \%-20 \%$ & \\
\hline Simple Payback Period & $1-7$ years & $\begin{array}{l}\text { Depends largely on commissioning costs, varies } \\
\text { by building type }\end{array}$ \\
\hline Ultimate Market Penetration & $8 \%-55 \%$ & $\begin{array}{l}\text { Very limited market penetration to date suggests } \\
\text { a value toward the lower end of the range }\end{array}$ \\
\hline Market Potential & $\begin{array}{c}4-30 \\
\text { billion } \mathrm{ft}^{2}\end{array}$ & \\
\hline $\begin{array}{l}\text { Market-Achievable Annual } \\
\text { Energy Savings }\end{array}$ & $\begin{array}{c}0.08-1.3 \\
\text { quad }\end{array}$ & \\
\hline
\end{tabular}

\subsubsection{Percent Peak Reduction}

Using daylighting to reduce artificial lighting levels reduces peak electric demand. A simple calculation suggests that an approximate $20 \%$ to $60 \%$ reduction in lighting energy during peak demand periods is possible. This corresponds to a peak reduction of approximately $6 \%$ to $18 \%$ as lighting accounts for about 30\% of peak demand in the commercial building sector (Nadel 2000, Brown and Koomey 2002).

\subsubsection{Payback Period}

The costs of these systems are not widely discussed in general, with only limited discussion about the added cost of dimmable ballasts in the popular literature. Other system costs include the photo sensors, controls, and labor for wiring and commissioning. PG\&E (2000) interviewed various contractors and established a very broad installed cost range of approximately $\$ 0.20-\$ 3.00 / \mathrm{ft}^{2}$, with sensor commissioning (photosensor placement and calibration) accounting for a significant portion (about $\$ 300$ to $\$ 600$ per sensor installation). They also identified a general unfamiliarity and lack of comfort with the installation

\footnotetext{
${ }^{1}$ For T8 bulbs (20,000-hour lifetime) and 1500 MA bulbs (14,000-hour lifetime), respectively; NEMA (1992).
} 
of photosensor-based lighting control systems among contractors, which contributes to higher costs. A very rough calculation of payback indicates that photosensor-based lighting control systems can pay for themselves in about 1 to 7 years. ${ }^{1}$ In practice, payback periods depend greatly on characteristics of the space (size, layout, light sources, etc.), the degree of over-lamping, and the wattage served by the sensor.

\subsubsection{Ultimate Market Penetration}

A one- to seven-year payback period corresponds to an ultimate market penetration of approximately $8 \%$ to 55\% based on the A market penetration curve (see the Appendix). There are non-energy benefits such as enhanced occupant comfort due to improved light quality (potentially increasing productivity or sales) that could increase market penetration; however, these potential impacts are not well quantified and are not considered in the market potential. Despite coming to market at least ten years ago, photosensorbased lighting control has realized very limited market penetration ${ }^{2}$ (PG\&E 2000) so the entire market remains for possible penetration, suggesting that the market potential may tend toward the lower end of the range.

\subsubsection{Demand Control Ventilation}

\subsubsection{Definition}

Minimum required outdoor air (OA) ventilation rates decreased in the early 1980s in response to high energy prices. These OA rates reduced energy bills by reducing the energy expended to condition the OA, but they led to the onset of sick building syndrome in buildings. To address this problem, OA rates were increased threefold in ANSI/ASHRAE Standard 62-1989, "Ventilation for Acceptable Indoor Air Quality.” Until recently, required OA ventilation rates scaled with the maximum occupancy of buildings; in practice, however, most real-time occupancy levels are less than the maximum design levels. Demand Control Ventilation (DCV) regulates the amount of OA coming into a building on the basis of varying occupancy levels.

ANSI/ASHRAE Standard 62 offers two options for maintaining adequate ventilation, the ventilation rate procedure and the indoor air quality (IAQ) procedure, and building codes throughout the United States are in the process of adopting it. The ventilation rate procedure uses the traditional prescriptive method, i.e., a minimum quantity of cubic feet per minute $(\mathrm{cfm})$ per person based on maximum occupancy. More recent changes to ASHRAE 62-2001, specifically addendum n, modify the ventilation rate procedure to include a non-zero ventilation rate at zero occupancy. In contrast, the IAQ procedure allows designers to vary the outdoor ventilation rate (from 0 to 100 percent of the design $\mathrm{OA}$ ) if the carbon dioxide $\left(\mathrm{CO}_{2}\right)$ level remains below a recommended level, i.e., $700 \mathrm{ppm}$ greater than outdoor $\mathrm{CO}_{2}$ levels. In this case, $\mathrm{CO}_{2}$ levels serve as a proxy for building occupancy and the rate of human-generated indoor pollutants, e.g., odors. Both procedures can enable DCV implementation, although proponents have primarily advocated a variant based on the ventilation rate procedure (e.g., Murphy 2002). Just as thermostats regulate the amount of cooling or heating supplied to a building space, $\mathrm{CO}_{2}$ sensors measure and regulate the amount of fresh air supplied to the space for buildings that use a DCV strategy.

\footnotetext{
${ }^{1}$ Assuming annual lighting energy consumption of $7 \mathrm{kWh} / \mathrm{ft}^{2}$ and an electricity cost of $\$ 0.08 / \mathrm{kWh}$.

${ }^{2}$ Rubinstein et al. (2000) estimated that controllable ballasts had only $\sim 2 \%$ market share and cost at least twice that of non-dimming ballasts.
} 


\subsubsection{National Energy Savings}

Demand Control Ventilation savings are shown in Table 2.13. If a DCV strategy calls for less outdoor air over the course of the heating and cooling seasons than does a prescriptive ventilation strategy, then the annual energy required to heat or cool the OA decreases. In addition, lower OA requirements decrease the fan energy expended to introduce and expel the air from the building in variable-air volume (VAV) systems. It is widely believed that actual occupancy levels in U.S. buildings are significantly lower than the design occupancy levels that conventional ventilation systems are set to handle. Field experience indicates that actual occupancy levels are at least $25 \%$ to $30 \%$ lower and perhaps as much as $60 \%$ to $75 \%$ lower in some buildings than design levels (Brandemuehl and Braun 1999; Turk et al. 1987). The ultimate energy savings in a given application depend on the actual (versus design) occupancy level patterns, as well as building type and climate (Roth et al. 2002; Brandemuehl and Braun 1999; Schell et al. 1998). Available data suggest that DCV reduces ventilation, heating, and cooling loads by $10 \%$ to $30 \%$, with a national average likely near the lower end of that range (Roth et al. 2002). Buildings and spaces with large swings in occupancy, e.g., movie theatres and conference rooms, tend to realize the largest savings.

Table 2.13. Summary of Demand Control Ventilation (DCV) Energy Savings

\begin{tabular}{|l|c|l|}
\hline Attribute & \multicolumn{1}{|c|}{ Value } & Notes \\
\hline Technical Market Size & $\begin{array}{c}\sim 55 \text { billion } \\
\mathrm{ft}^{2}\end{array}$ & Most conditioned commercial floor space \\
\hline $\begin{array}{l}\text { Relevant Annual Energy } \\
\text { Consumption }\end{array}$ & 5.4 quads & All HVAC energy \\
\hline Energy Savings & $10-15 \%$ & $\begin{array}{l}\text { Approximate average; large variations depending on } \\
\text { actual versus design occupancy level patterns, } \\
\text { building type, and climate }\end{array}$ \\
\hline $\begin{array}{l}\text { Technical Annual Energy } \\
\text { Savings Potential }\end{array}$ & 0.5 quads & \\
\hline $\begin{array}{l}\text { Commercial Building Peak } \\
\text { Reduction }\end{array}$ & $10-15 \%$ & $\begin{array}{l}\text { Approximate average; large variations depending on } \\
\text { actual versus design occupancy level patterns, } \\
\text { building type, and climate }\end{array}$ \\
\hline Simple Payback Period & $2-3$ years & $\begin{array}{l}\text { Can vary more depending on specific building } \\
\text { occupancy patterns. }\end{array}$ \\
\hline Ultimate Market Penetration & $15-30 \%$ & For 2 to 3-year payback period \\
\hline Market Potential & $\begin{array}{l}8-17 \\
\text { billion } \mathrm{ft}^{2}\end{array}$ & \\
\hline $\begin{array}{l}\text { Market-Achievable Annual } \\
\text { Energy Savings }\end{array}$ & $\begin{array}{c}0.08-0.25 \\
\text { quads }\end{array}$ & \\
\hline
\end{tabular}

Currently, most buildings do not use DCV for a range of reasons. Although $\mathrm{CO}_{2}$ levels tend to correlate well with human occupancy and human-generated pollutants, they do not reflect the buildup of nonoccupancy-related pollutants, such as fumes from copiers and printers, out-gassing from building materials, carpets and furniture; and vapors from cleaning supplies. In general, building operators have greater concerns about IAQ than building energy consumption, lowering their interest in decreasing outdoor air flow. Moreover, the large number of addenda to and interpretations of ASHRAE 62-2001 may have increased (instead of allayed) concerns about whether or not DCV implementations satisfy IAQ requirements. 
Building operators using the IAQ procedure need to ensure that all potential contaminants remain at safe levels. As a consequence, contractors and designers have concerns about liability for systems that do not meet IAQ standards when under the IAQ procedure. This could occur because one of the numerous nonhuman indoor pollutants rises above acceptable levels or because of improper $\mathrm{CO}_{2}$ sensor installation or sensor failure. In contrast, the ventilation rate procedure offers less room for liability because it is deemed to provide acceptable IAQ (ASHRAE 2001). DCV also is a new concept for standards, and local building codes have been slow to adopt it. Additional permitting and verification is often required if they do adopt it. Other issues discouraging widespread DCV adoption include the need for savvy system installation and knowledgeable operational personnel, which cost more and are hard to find; $\mathrm{CO}_{2}$ sensor cost and maintenance issues; and the limited number of control systems that support $\mathrm{CO}_{2}$ sensor input for ventilation control (Roth et al. 2002).

\subsubsection{Percent Peak Reduction}

DCV reduces peak electricity demand when actual occupancy levels fall below design occupancy levels during peak demand periods. Lower levels of OA translate into decreased cooling (and, to a lesser extent, ventilation) loads and, therefore, air-conditioning power draw. Under the IAQ procedure, DCV may allow building operators to close fresh air dampers for short periods during the hottest hours in the summer (typically coinciding with peak electric load). In general, peak reductions vary from building to building, depending on occupancy patterns. Thus, peak demand reduction will likely mirror the cooling energy savings potential range, i.e., 10 to $30 \%$ of HVAC energy consumption, or $5 \%$ to $15 \%$ of total commercial building peak demand, with the national average closer to $5 \%$.

\subsubsection{Simple Payback Period}

The $\mathrm{CO}_{2}$ sensors required to implement DCV cost approximately $\$ 400$ to $\$ 500$ a piece (installed), with typically one sensor installed per zone $\left(\sim 2,000\right.$ to $\left.3,000 \mathrm{ft}^{2}\right)$. Additional expenses will likely be required to integrate the sensors into building controls (Schell and Int-Hout 2001; Roth et al. 2002). In practice, DCV has reduced annual energy costs by $\$ 0.05$ to $\$ 1.00$ per square foot, with large variations reflecting the range in building types studied. On average, DCV appears to have a 2-to-3-year payback period (Roth et al. 2002).

\subsubsection{Ultimate Market Penetration}

Two- and three-year payback periods correspond to ultimate market penetrations of approximately 30\% and $15 \%$, respectively, yielding annual national energy savings of 0.08 to 0.25 quads.

\subsection{Market Barriers}

Building controls are currently realizing only a fraction of their energy saving potential. For example, only about 33\% of commercial floorspace is served by an EMCS and most of this appears to realize only a portion of its energy savings potential. In addition, the commissioning rate of new commercial buildings languishes below 5\% (Engineered Systems 1999). Advanced controls concepts such as integrated building systems have, for the most part, a negligible market share. Each of the specific control approaches discussed above faces barriers to achieving greater market share that are specific to that approach. Nonetheless, the approaches share common barriers related to the buildings market and other factors. 


\subsubsection{Minimal Concern for Energy Costs}

A central issue with all energy savings measures is that energy costs simply do not represent a significant portion of expenditures for most buildings and buildings owners and tenants typically care little about energy expenditures. For instance, one study found that energy expenditures account for just over 1\% of total annual expenditures for a medium-sized office building (see Table 2.14).

Table 2.14. Breakdown of Typical Small Office Building Annual Expenditures (from Cler et al. 1997)

\begin{tabular}{|l|c|}
\hline \multicolumn{1}{|c|}{ Expenditure } & Annual Cost, $\mathbf{\$} / \mathbf{f t}^{\mathbf{2}}$ \\
\hline Office-Workers' Salaries & 130 \\
\hline Gross Office Rent & 21 \\
\hline Total Energy Use & $1.81^{(\mathrm{a})}$ \\
\hline Electricity Use & 1.53 \\
\hline Repair and Maintenance & 1.37 \\
\hline Space Cooling and Air Handling Electricity & $0.61^{(\mathrm{b})}$ \\
\hline Space Cooling and Air Handling Maintenance & 0.82 \\
\hline Total Building Operations and Management Salaries & 0.58 \\
\hline $\begin{array}{l}\text { (a) } \text { Dougan and Damiano (2003) note a range of } \$ 1.00 \text { to } \$ 2.00 / \mathrm{ft}^{2} \\
\text { (b) for office buildings. }\end{array}$ \\
\hline
\end{tabular}

Energy expenses may equal a higher percentage of building operating expenses in other kinds of buildings, such as retail or food sales. In those cases, energy efficiency measures compete directly with funds that could be invested in core business functions, e.g., enhanced lighting or displays that can increase sales of clothes or food. Consequently, building owners and/or operators must have very high levels of confidence that building controls investments will have a quick payback for those investments to prove attractive. Institutional parties such as governments, schools, and some hospitals, may accept somewhat longer (up to $\sim 5$ years) simple payback periods.

\subsubsection{First (Capital) Cost}

First cost also appears to be a major factor for the particularly low penetration of EMCSs in smaller commercial buildings. As shown in Figure 2.2, the likelihood of having an EMCS installed correlates very strongly with building floorspace, e.g., a building with $150,000 \mathrm{ft}^{2}$ has about a ten-fold higher probability of having an EMCS than one with less than 10,000 $\mathrm{ft}^{2}$. For larger buildings, the first cost of an EMCS, as well as the expense of personnel to operate and maintain the EMCS, accounts for a smaller portion of total building expenditures than in smaller buildings and makes the EMCS a more attractive investment.

Building owners often see first cost as a more significant issue for more advanced building controls approaches than in conventional systems. New integrated or "smart" buildings generally cost more to build due to greater system integration and the need for more control and measurement points. Incorporating building integration into existing buildings requires installation of the sensor and communications infrastructure on top of existing building systems. This can prove "quite prohibitive.” Replacing existing pneumatic controls with DDC systems "can add exorbitant cost." Similarly, the cost of integrating separate building systems into a single BAS "can be quite high due to the need for communication gateways and revised software” (Energy Design Resources 2001b). Not surprisingly, a survey carried out by BOMA (2000) revealed that cost dominates decisions to not implement systems integration (Table 2.15). 
Table 2.15. Top 5 Reasons Building Owners Do Not Implement Building Systems Integration (from BOMA 2000)

\begin{tabular}{|l|}
\hline \multicolumn{1}{|c|}{ Reason } \\
\hline 1. High Installation Cost \\
\hline 2. Lack of Cost Justification \\
\hline 3. High Systems Integration Cost \\
\hline 4. Lack of Funding \\
\hline 5. Lack of Awareness \\
\hline
\end{tabular}

Building owners also view novel building controls as carrying greater financial risk than conventional controls measures, due to their relative immaturity, poorly understood cost and a general skepticism about purported cost savings. As the “Technology Roadmap for Intelligent Buildings” prepared by the Continental Automated Buildings Association found, most intelligent building projects have not been fully instrumented and documented. This prevents meaningful quantification of the costs and benefits of “intelligent” buildings (CABA 2002). Establishing a meaningful cost-benefit relationship for novel building controls - such as integrated building systems - is a vital part of gaining building owner confidence so that novel building controls can achieve significant market penetration.

\subsubsection{Building Ownership and Management}

The way that buildings are managed and built has a major impact on buildings decisions, including those related to building energy consumption (Reed et al. 2000). For existing buildings, the building management model has a dominant impact on who the key decision-makers are and what factors make different investment choices attractive. For new construction, the building ownership-model and new construction practice both strongly influence decisions.

The willingness of firms to invest in efficiency measures depends on what the owner plans to do with the building (Reed et al. 2002). If they intend to buy, renovate, and sell the building, the firm will focus on measures that increase the selling price (and thus the lease rate) of the property. Efficiency measures that do not enhance the property's value need to recoup their cost within (or less than) the expected time before sale of the building, typically from a few months to three years.

If, on the other hand, the owners plan to hold the building instead of selling, the investment horizon increases substantially, perhaps to as long as about five years (Reed et al. 2002). In the buy/renovate/hold case, several different parties impact investment decisions. Tenants can drive changes in the leased space they want (the costs of which are recovered through the lease); these changes tend to focus on reducing complaints from employees and improving employee comfort and/or productivity, e.g., layout modification and lighting. The building owner typically recovers his investment through the lease.

\subsubsection{Common Building Construction Paradigms}

The dominant new construction process paradigms for commercial buildings tend to impede the effective deployment of building controls, particularly novel building control concepts such as integrated building systems. Table $\mathbf{2 . 1 6}$ shows an overview of the three models for new construction.

\footnotetext{
${ }^{1}$ Presumably, of the benefits of building system integration.
} 
Table 2.16. Models for New Construction (based on Reed et al. 2000)

\begin{tabular}{|c|c|c|c|}
\hline $\begin{array}{c}\text { Construction } \\
\text { Model }\end{array}$ & $\begin{array}{l}\text { Approximate } \\
\text { Market Share }\end{array}$ & Characteristics & $\begin{array}{c}\text { Barriers to Implementing } \\
\text { Building Controls }\end{array}$ \\
\hline $\begin{array}{l}\text { Plan / Design } \\
\text { / Build }\end{array}$ & $<\sim 45 \%$ & $\begin{array}{ll}\text { - } & \text { Architect integrates } \\
\text { building construction } \\
\text { process } \\
\text { - } \quad \text { Longer construction time } \\
\text { than Design/Build }\end{array}$ & $\begin{array}{l}\text { Opportunities for more } \\
\text { integrated controls } \\
\text { approaches depend on } \\
\text { architect }\end{array}$ \\
\hline Design/Build & $50 \%+{ }^{1}$ & $\begin{array}{ll}\text { - } & \text { Reliance on standard / pre- } \\
& \text { existing designs } \\
\text { - } & \text { Shorter construction time }\end{array}$ & $\begin{array}{ll} & \text { Little opportunity for } \\
\text { integrated approaches } \\
\text { - } & \text { Avoidance of innovation } \\
\end{array}$ \\
\hline Collaborative & $4-8 \%$ & $\begin{array}{ll} & \text { Systems approach used } \\
\text { - } & \text { Longer construction time } \\
\text { than other paradigms }\end{array}$ & $\begin{array}{l}\text { - Excellent opportunity for } \\
\text { "whole buildings" } \\
\text { approaches }\end{array}$ \\
\hline
\end{tabular}

Plan/Design/Build is often used for public, owner-occupied, and buildings with complex function. In this model, the building owner selects an architect through a competitive process and the architect develops a detailed building design, often with help from specialist subcontractors. Thus, the owner and architect drive the process and make key decisions. The ability to implement more novel controls approaches, most notably integrated approaches, depends on - and varies greatly with - the ability of the architect to manage the different teams working on the building and to successfully exchange information between them. Once the primary model for new construction, the new construction market has moved away from plan/design/build and toward Design/Build due to cost and time constraints (Reed et al. 2000).

The Design/Build model centers on a building contractor selected by the building owner to design and then construct the building. To a large extent, the building design and construction are worked out independently of each other and in sequence. This approach fixes many design variables early on in the process, enabling different parts of the construction processes to overlap. While this approach expedites construction, it can constrain portions of the design decided later in the process significantly.

Consequently, contractors tend to re-use structural elements from building to building (with some siterelated variation) and design work is often formula- and rule-of-thumb-driven. All of these factors make whole building design and controls approaches very difficult to implement. ${ }^{2}$ Presently, at least $50 \%$ of all new construction projects appear to follow the Design/Build model and its market share continues to grow (Reed et al. 2000).

In contrast to the other two paradigms, the Collaborative approach looks at buildings as a system and strives to build a high-quality, well-integrated building. Realizing an integrated building in practice requires extensive communication and sharing of information between all of the parties involved in the building process to allow effective consideration and exploitation of system interactions (e.g., via modeling techniques). Because it takes a whole building view of the building, the collaborative model has a large potential for achieving energy efficiency, including the use of integrated building systems. Due to the need for extensive up-front design integration and continued information sharing, the collaborative

\footnotetext{
${ }^{1}$ Sinclair (2003) supports this estimate.

${ }^{2}$ For instance, fire protection system typically must be installed and fully operational before building occupancy (Brown 1998). If the HVAC system integrates with the fire protection system, it, too, must be completely installed before installation to demonstrate that it does not adversely impact fire protection system function.
} 
model typically has a higher first cost and takes longer to construct than design/build and plan/design/build. Currently, it accounts for roughly between $4 \%$ and $8 \%$ of projects (Reed et al. 2000).

\subsubsection{Building Codes and Standards}

Conflicts with or ambiguity about building codes and standards may impede the deployment of novel building control systems. In particular, fire codes appear to pose a significant barrier to full integration of building systems. Fire and life safety systems can include many sensors and can be quite sophisticated e.g., some incorporate automated response sequences when a fire occurs (annunciation of fire, smoke pressurization). Because they are responsible for protecting the building occupants from fires, fire protection systems need to have a very high degree of reliability and integrity to ensure function in emergency situations, even when other systems fail. Consequently, they require isolation from potential interference from other building systems; fire systems can provide information to - but cannot receive information from - other building systems, limiting integration possibilities.

In addition, the National Electrical Code requires that conductors remain separate from communication and power circuits to prevent interference (Brown 1998). In principle, any building control system that meets the requirements of NFPA 72 can serve as a fire system (McGowan 1995). This also requires compliance with UL 864 "The Standard for Fire Alarm System Control Units,” which requires (among other things), that microprocessor-based fire alarm systems store control logic in nonvolatile memory (Brown 1998). According to Bushby (2001), proper system design practice should be able to overcome the integrity concerns to enable greater integration.

\subsubsection{Knowledge and Understanding of Controls}

Many of the problems encountered with EMCSs occur due to a relatively low level of understanding of building controls and systems. The general lack of knowledge of building controls clearly manifests itself during the development of EMCS specifications, which often are not application-appropriate and result in selection of an inappropriate system (Santos and Brightbill 2002). Inadequate EMCS specifications also adversely impact system interoperability. For example, Santos and Brightbill (2002) note that many systems are not specified to the level needed for the context, i.e., simply demanding adherence to an open protocol does not result in an interoperable systems. Furthermore, many control systems lack full documentation of the system in its operational context, i.e., such that the operator has sufficient explanation of design intent to understand the system and all information needed to maintain (i.e., reprogram as needed) the system.

The rapid evolution of the building controls field (discussed in the "Introduction" Section) and increasing complexity of building controls are overarching challenges that exacerbate a general lack of knowledge. Table 2.17 summarizes how relevant parties suffer from understanding gaps specific to their position and how this adversely impacts the energy efficacy of building controls. 
Table 2.17. Impact of Understanding Gaps of Key Control Parties on Building Controls Energy Performance Shortfall (based on Barwig et al. 2002 and other sources)

\begin{tabular}{|l|l|l|}
\hline Party & Understanding Gap & Reason for Energy Performance Shortfall \\
\hline Building Owners & $\begin{array}{l}\text { Pros and cons of different } \\
\text { control systems, components }\end{array}$ & $\begin{array}{l}\text { Purchasing decisions based on first cost } \\
\text { (minimal product differentiation) }\end{array}$ \\
\hline $\begin{array}{l}\text { Control System } \\
\text { Designers }\end{array}$ & $\begin{array}{l}\text { Impact of control strategies on } \\
\text { energy consumption }\end{array}$ & $\begin{array}{l}\text { Energy-efficient control strategies not } \\
\text { considered and specified }\end{array}$ \\
\hline $\begin{array}{l}\text { Control System } \\
\text { Specifiers }\end{array}$ & Optimal sensor placement & $\begin{array}{l}\text { Sensors cannot provide the most useful or } \\
\text { appropriate information to EMCS }\end{array}$ \\
\hline EMCS Operators & $\begin{array}{l}\text { Control procedure intent, } \\
\text { EMCS operation }\end{array}$ & $\begin{array}{l}\text { Limited repertoire of operating procedures } \\
\text { leading to inadvertent energy waste }\end{array}$ \\
\hline
\end{tabular}

The general nature of the buildings controls industry also works against full exploitation of the potential of buildings controls. As noted earlier, building controls have evolved greatly over the past couple of decades, with major changes in all facets of the business. Keeping up with the changes - let alone taking advantage of - the new opportunities they afford requires talented workers. An executive in the fire protection industry argues that the current industry structure does not support the employment of such personnel:

"Modern building systems are computer systems, and the vendors are competing with all other aspects of the information technology industries for qualified technicians. These technicians are expected to know the hardware and software of the control system, as well as all relevant codes, standards, and industry practices related to HVAC control, fire alarms, elevator control, security, lighting control, etc. This is an unreasonable expectation considering industry pay scales, training, turnover, and service call charge rates” (Brown 1998).

This parallels long-running complaints from the HVAC industry about the difficulty of finding qualified good technicians.

\subsubsection{Experience with Existing Building Controls}

Success in the field is very important to establishing the credibility and ultimate market success of energy efficiency measures, including building controls. If a building controls approach falls substantially short of promised energy savings levels, the resulting credibility gap inhibits further deployment of the approach. A substantial portion of EMCSs do not realize most of their energy-savings potential. Case studies and anecdotal accounts abound for the underlying reasons building controls do not realize their full energy savings potential; however, a widely inclusive literature review of building controls problems from 67 case studies, encompassing 118 buildings, appears to offer the most concrete insight into this shortfall (Ardehali and Smith 2002). Based on the information available in the case studies, they categorized the primary causes of energy performance shortfalls as software (32\%); human factors (29\%), and hardware (26\%). In $13 \%$ of the cases, the cause could not be determined. ${ }^{1}$

A related effort (Barwig et al. 2002) surveyed building controls industry experts in an attempt to develop a more refined assessment of building controls problems. At a top level, the survey results validate the primary problems identified in the literature survey; in addition, they provided a qualitative estimate of the energy impact of different problems (see Table 2.18).

\footnotetext{
${ }^{1}$ Ardehali and Smith (2002) indicate that "the majority of the non-specified problems reviewed include malfunction or improper operation of the economizer."
} 
Table 2.18. Barriers Impeding EMCS Energy Savings and Operation, Ranked by Prevalence and Energy Impact (from Barwig et al. 2002)

\begin{tabular}{|c|c|c|c|c|}
\hline Problem & Type & Description & $\begin{array}{l}\text { Relative } \\
\text { Preva- } \\
\text { lence }\end{array}$ & $\begin{array}{l}\text { Relative } \\
\text { Energy } \\
\text { Impact }\end{array}$ \\
\hline Input Device & Hardware & Sensors, transducers, wiring & High & High \\
\hline Controller & Hardware & $\begin{array}{l}\text { Controller hardware/component } \\
\text { problems/failure }\end{array}$ & Low & Low \\
\hline $\begin{array}{l}\text { Controlled } \\
\text { Device }\end{array}$ & Hardware & $\begin{array}{l}\text { Valves and dampers (and their } \\
\text { operators), electric relays, fans, pumps, } \\
\text { compressors, VSDs }\end{array}$ & Medium & High \\
\hline Communications & Hardware & Data transmission hardware & Low & Medium \\
\hline $\begin{array}{l}\text { I/O } \\
\text { Implementation }\end{array}$ & Software & $\begin{array}{l}\text { Problems with control software arising } \\
\text { prior to building end user receipt of } \\
\text { system (e.g., point addressing) }\end{array}$ & High & High \\
\hline Programming & Software & Incorrect or inappropriate control logic & High & High \\
\hline Operation & Software & $\begin{array}{l}\text { Arise after building start-up (e.g., loss } \\
\text { of setpoints from power outage) }\end{array}$ & Medium & Medium \\
\hline $\begin{array}{l}\text { Data } \\
\text { Management }\end{array}$ & Software & Data monitoring, display, archiving, etc. & Low & Low \\
\hline Operator Error & $\begin{array}{l}\text { Human } \\
\text { Factors } \\
\end{array}$ & $\begin{array}{l}\text { Unintentional changes to control system } \\
\text { during system operation or maintenance }\end{array}$ & High & High \\
\hline $\begin{array}{l}\text { Operator } \\
\text { Unawareness }\end{array}$ & $\begin{array}{l}\text { Human } \\
\text { Factors }\end{array}$ & $\begin{array}{l}\text { Lack of operator understanding, } \\
\text { operator ignorance }\end{array}$ & Medium & High \\
\hline $\begin{array}{l}\text { Operator } \\
\text { Interference }\end{array}$ & $\begin{array}{l}\text { Human } \\
\text { Factors }\end{array}$ & $\begin{array}{l}\text { Intentional modification of control } \\
\text { systems }\end{array}$ & High & High \\
\hline $\begin{array}{l}\text { Operator } \\
\text { Indifference }\end{array}$ & $\begin{array}{l}\text { Human } \\
\text { Factors }\end{array}$ & $\begin{array}{l}\text { Operator apathy towards building } \\
\text { system operation or maintenance }\end{array}$ & Low & Medium \\
\hline
\end{tabular}

\subsection{Value Proposition for Building Controls}

\subsubsection{Enhancing the Indoor Environment and Building Economic Activity}

Initially, EMCSs were installed to reduce operations and maintenance costs. When energy prices skyrocketed during the 1970s, reducing energy costs became a valuable function. Nonetheless, maintaining occupant comfort ranks as the foremost goal of buildings operations. Indeed, a survey of EMCS operators found that the overwhelming majority cited "control of comfort" as their primary objective (Lowry 2002), which coincides with a similar survey of office tenant priorities (BOMA 1999). Clearly, comfort impacts tenant retention (McGowan 1995). On the other hand, only one operator rated "Cost Saving” as the top requirement. Consequently, enhancing occupant comfort ${ }^{1}$ and productivity rank as the primary value propositions for controls, with cost-effective reductions of operating and maintenance expenses as a second potential proposition.

The dominance of worker salaries in an office setting (see Table 2.14 above) suggests that building controls investments that enhance the productivity of workers, even by only $1 \%$ or $2 \%$, would be very

\footnotetext{
${ }^{1}$ In many instances, simply granting occupants the possibility of controlling their environment improves their comfort; this includes the perception that they are controlling their environment, e.g., the non-functional thermostat examples described by Checket-Hanks (2003).
} 
attractive investments. ${ }^{1}$ Many lighting professionals see this as the key driver for greater installation of lighting controls. Similarly, a building EMCS that results in a more enjoyable working environment may reap value by increasing employee retention. In retail or food service settings, if building controls can improve the indoor environment and thus increase sales even if by only a relatively small percentage, they would make an attractive investment for those applications. In all cases, building controls can greatly increase their value by enhancing the core business of the building - be it office employee productivity or increased sales. All parties benefit from a more productive environment. The building occupants realize the aforementioned gains and the lessor can command more rent for his property.

Prior research suggests a general relationship between building factors related to controls, e.g., personal climate control, operable windows, etc. (see Fisk 2000, Wyon 2000). Although building tenants appear to place a high value on measures related to occupant comfort (BOMA 1999), it remains for the owner or operator to link tenant comfort to financial parameters such as productivity. Without this link, it is difficult to make a convincing business case for substantial investment (Reed et al. 2000). The magnitude of the potential value from increased employee productivity provides the motivation for further research to understand and document the productivity linkage to lighting, environment control, IAQ, etc. Some studies suggest that simply granting occupants the ability (or even the perceived ability) to control their environment can realize meaningful improvement in occupant comfort and productivity (Wyon 2000).

\subsubsection{Reducing Building Maintenance and Operations Expenses}

Building controls can be sold - and were initially installed - as a way to reduce building maintenance and operations expenses, if the owner can be convinced of the cost-effectiveness of this measure. The value proposition appears stronger in the centralized case, i.e., EMCS. For instance, centralized DDC control allows the EMCS operator to make temperature set point changes in all zones via the EMCS, whereas pneumatic or local building controls would require manual modification at each terminal unit. In another case, a hospital with a new EMCS (upgraded from a circa 1993 DDC system) that collects data from over 100 nodes has enabled building operations personnel to remotely monitor and access the data. The net impact: the operators now perform preventive maintenance based on information from the EMCS. They experienced a "substantial" decrease in trouble calls and labor costs decreased by approximately $50 \%$ (ACHRN 2002).

As discussed in Section 2.5, building controls can reduce building energy consumption. Even though energy expenditures generally are not a major concern of building owners, building controls investments with reliable and short payback periods can prove attractive. In many cases, utility policies and pricing structures have a strong impact on the attractiveness of control economics, primarily through demand charges and, to a lesser extent, time-of-use metering and interruptible power contracts. Effective application of real-time pricing could provide greater incentive for controls that attain rapid reductions in peak electricity demand in response to real-time cost signals received from utilities. Ideally, building control systems would obtain real-time price data (see Section 3) and incorporate these data into building control decision making. For example, software products that interface with EMCSs have begun to come to market that include global optimization algorithms that alter building operations to minimize operating cost function within certain comfort constraints.

\footnotetext{
${ }^{1}$ See, for example, Fisk (2000) for more information.
} 


\subsection{CURRENT APPLICATIONS AND STRATEgIES FOR NEW APPLICATIONS}

\subsection{Overview}

Proper control of building systems is essential to maintain the most efficient systems operation, sustain the highest level of indoor environmental quality, and respond appropriately to external forces. Current practices fall short of achieving optimal operations due to the disparate nature of the building industry and a lack of understanding of how complex building systems interact with their environment. Work on the control strategies used in buildings is needed to optimize operation while keeping them flexible and straightforward. Case studies with hard data on the performance of these systems are needed to document what works and what needs refinement. ${ }^{1}$

Several themes emerged from this chapter:

- Integration topic areas are multidisciplinary in nature.

- Technical case studies, such as those funded by DOE on high performance buildings,2 that collect "hard-data" from real buildings are needed to prove that the technologies work.

- The amount of control is limited to the ability to provide input to the system. The motto is "you cannot control better than you can measure." In some cases, sensor deficiency can be overcome with appropriate algorithms.

By advancing controls technology in the application of controls, the following benefits will be seen by the building sector:

- Help buildings (and related equipment) meet their full potential to save energy and manage (electrical) demand

- Provide for the effective integration of new technologies

- $\quad$ Provide better control of indoor environmental quality (IEQ).

\subsection{Scope and Organization}

The chapter discusses issues related to the control instructions and algorithms that are to be implemented through control systems. The range of buildings includes both residential and commercial applications (with the main focus on commercial buildings). This section addresses current applications and the barriers for these applications as well as identifying why current control systems are not reaching their full benefit in terms of usability and minimizing energy consumption in buildings. Additionally, this section documents the important areas of research to get buildings to operate at their full potential. It is understood that the definition of "full potential" will change depending on the location, building owner, and type of building. Determining exactly how systems, such as HVAC, lighting, and yet-to-bedetermined technologies, should be controlled and integrated is the focus of this section.

\footnotetext{
${ }^{1}$ Editors note: The Buildings Technology (BT) program has funded and collected a series of case studies on High Performance Buildings. These studies are available at: http://www.eere.energy.gov/buildings/highperformance/case_studies/ 2 Ibid.
} 
This chapter is organized into subsections consisting of individual topic areas. The topic areas are based on technologies, strategies, or points of view. Each subsection is organized in the same manner with three sections: 1) background related to the need for the topic, 2) the current state of the topic, and 3) research and development that is needed to advance technologies related to the topic.

\subsection{Traditional HVAC Controls}

\subsubsection{Background Needs}

There is a considerable amount of inertia in the building controls industry and therefore a practical need to develop newer "advanced” controls that are based on existing capabilities. Much of today's controls and related algorithms are based on pneumatic systems and the level of algorithms possible with this type of control. Some work has been done to develop controls for standardized modules, such as VAV boxes, chillers, and variable speed drives. Currently, there is a need to improve the interaction of these components to adapt to systems outside of their own sub-system. Experience shows that there are significant problems associated with setting up controls for new, innovative HVAC systems from both hardware and algorithm development points of view.

\subsubsection{Current Status}

The private sector continually incorporates new products into existing control systems. The rate of development is slow and reincorporating non-traditional strategies often requires time-consuming configuration and custom programming. Most new controllers are limited to a single device.

Nevertheless, the fact that new products and systems are being introduced shows incremental change that is replacing existing technology, but not necessarily changing the methods.

The technology gap is that research-level control technology algorithms do not match with current applications. This is true both at the HVAC component level as well as at the overall systems integration level.

\subsubsection{Proposed Research}

1. Study processes currently used in the controls industry with focus on how advanced controls can best be incorporated into the industry and potential for interaction between building components.

2. Develop new algorithms that "hang" onto existing control systems. These algorithms can be based on pre-determined rules approaching an optimum or real-time optimal controls of hardware.

3. Develop outside air controls based on real-time measurements of indoor air quality (mainly $\mathrm{CO}_{2}$, $\mathrm{CO}$, humidity, and in some cases VOCs) with the benefit of an economizer.

4. Develop human-machine interfaces at appropriate levels. These range from building occupants to building operators-each with their own needs for information and level of control. A common complaint is that these interfaces are only for trained technicians, making them "too complex" to the average building user.

5. Develop global control schemes that approach optimum performance.

6. Develop methods that drastically reduce installation cost, improve serviceability, and reduce maintenance needs of the control systems. 
7. Develop smart air terminals and HVAC systems in a building network environment that recognize when new components are added or when the performance of existing components is compromised and adjust the control strategy accordingly.

8. Develop HVAC systems model-based design tools for use in specifying the most effective EMCS strategy. At the HVAC system level, these tools would be used to determine the sizing of HVAC components and airflows. At the whole building level, these tools would be used to explore the impacts of alternative HVAC technologies, configurations, and control strategies on buildings. These tools should further be incorporated into HVAC simulators for use in training HVAC installers, consulting engineers, and building operators.

\subsection{Lighting Controls}

\subsubsection{Background Needs}

Advanced lighting controls have huge energy savings potential. To achieve savings on lighting systems, it is well known that, the daylight must be harvested - the electricity used by lights must be reduced while maintaining minimum light levels. Currently, buildings can be designed to be daylit and technologies exist to dim and turn off lights.

The technology gap is that motion sensors do not provide determination of occupancy and lighting sensors do not assess the "lighting quality" of a space. Lighting quality is defined/perceived by the occupant of the space, but includes lighting intensity, distribution of lighting, spectrum of the lighting, and lighting contrasts (including glare). In addition, most open plan offices have varying light needs, poorly defined individual lighting zones, and large spatial variations in daylight levels. Improved sensors are needed for detecting motion and lighting quality.

From a controls application point of view, there are issues surrounding integration that go beyond conventional daylight controls. Using occupancy sensors to characterize and predict occupancy patterns could provide new methods of determining efficient set points for lighting (as well as HVAC). Providing cost-effective zoning of light fixtures and simple human interfaces for local control would improve this area of the building automated system (BAS). In addition to controlling electric lighting, there will likely be growth in the dynamic control of daylight entering through fenestration by the use of switchable glazings and/or actively controlled interior or exterior blinds.

From a demand perspective, having the ability to change set points based on an electrical demand priority could have large cost savings implications. Prioritizing this type of control is an area for research.

\subsubsection{Current Status}

There are a number of manufacturers that make motion sensors and light sensors. In addition, much work has been done to design daylit buildings and integrate daylighting controls. There has been a wide range of energy savings, but most projects with large energy savings also come with large sensor, control, implementation, and maintenance costs (Pless and Torcellini 2002)

\subsubsection{Proposed Research}

Research is proposed in the following areas:

1. Ability to easily bind sensors to lighting fixtures. 
2. Ability for occupancy patterns to be developed and used to better predict occupancy, rather than motion.

3. Determination of set points based on lighting quality needed, rather than lighting level over the entire spectrum.

4. Development of user interfaces that couple automation with human needs for lighting control.

5. Lighting interactions with demand responsive systems.

6. Interaction of lighting systems with HVAC control set points.

\subsection{Cost Implications for Applications}

\subsubsection{Background Needs}

Algorithms and instructions for controls need to be efficient so that system complexity and associated costs can be kept low. Economic issues are discussed more fully in another chapter, but the importance of cost cannot be overlooked in any aspect of such research. Typically, building controls are custom applications that are built up from a set of components. Wiring costs, system design costs, and custom programming costs prevent whole building control systems from being implemented in many buildings. The selection of control strategies will influence the complexity of the control system in terms of processing power and number of nodes. There is a need to characterize control algorithms not only in terms of how well they might improve performance of a building, but also how easily they can be implemented in a control system. If the costs associated with programming or implementing a certain control strategy are too high, then simpler strategies may need to prevail.

\subsubsection{Current Status}

Little work is being done to reduce the complexity and cost of these systems. New technologies seem to make the systems more complicated, rather than less complicated. Current control systems are largely custom configurations, both in hardware and in software. Some user interfaces employ graphical techniques, but extensive training is still needed to use them. Maintenance staffs can be reluctant to adopt the technologies - and if they do, there is a significant learning curve.

Real-time pricing strategies have received a lot of attention from the utility view, but less from the building controls' point of view. Some buildings do respond to current rate structures, but with custom programming based on past experience of the building operation. In general, most customers do not respond to external forces, unless they are catastrophic. Demand limiting strategies are limited and not all building owners may be aware of their rate structures. Some utilities have deployed radio-based controllers to shed major loads, but it is often done without customer control. In addition, customer interaction with utility meters is limited-this includes direct connections from the meter to the control systems. This if further complicated by the fact that many control systems are not data acquisition systems - they collect data and use it without storing it.

\subsubsection{Proposed Research}

1. Research is needed to characterize the efficiency of different control strategies in terms of the resulting control system costs. Data are needed to understand the balance between investment in controls (system complexity) and the return on that investment in terms of realized energy 
savings. Analysis should be flexible so that algorithm developers and control systems designers can adjust their assessment of control strategies as costs fluctuate.

2. Assuming that sensors and protocols are developed to provide data on factors outside the building (e.g., electricity prices, weather), research is needed to identify how to best use such information in building control decisions, including operation strategies and dynamic response to external market forces.

3. Research is needed to address any remaining barriers to cost-effective application of ECMS in smaller buildings. Demonstration projects are recommended that address first cost issues, take into account the building ownership and management models and educate building operators on the use of advanced controls.

4. Research is needed to establish a strategy for building retrofits that will achieve substantial national energy savings. Existing buildings account for 75\%-80\% of new building control system installations.

\subsection{Disaster Minimization and Mitigation}

\subsubsection{Background Needs}

Buildings may face disasters such as fire, power outages, and deliberate attacks; among others. Buildings are currently required by codes to have systems dedicated to control smoke and fire that typically respond by sprinkling and quickly altering building air systems to prevent spreading. The 9-11 terrorist attacks have raised concerns that buildings must have the capability to respond appropriately to chemical and biological attacks. HVAC air systems and fire dampers are considered important resources to deploy in mitigating such attacks, but the best use of these systems is not well understood. Utility grids have historically been very reliable, but limited transmission availability, boom and bust power generation investment cycles, volatile markets, and attacks on infrastructure will all tend to lower the reliability and quality of grid power in the future. This makes it more attractive to design buildings that can respond well to grid failures.

\subsubsection{Current Status}

Disaster mitigation has been studied from a risk management point of view (ASHRAE 2003).

Development of new sensors for chemical and biological weapons is ongoing. Techniques have been developed for modeling airflow and contaminant transport. Distributed generation is being developed that can enable buildings to provide their own power. Photovoltaic (PV) systems have been bundled with uninterruptible power systems (UPS) to provide emergency power during grid failure and demand reduction under normal operation (Warwick et al 2003). During an emergency, building systems will need to execute a coordinated response that includes efficient egress of the building population given accurate information about the status of evacuation routes.

\subsubsection{Proposed Research}

Research is needed to address how advanced control systems can best respond to disasters in the following areas:

1. Develop strategies for HVAC and fire control systems to respond to chemical and biological attacks. 
2. Develop methods of coupling airflow and contaminant transport models with building systems and the impacts of control options on short time scales.

3. Develop modeling and analysis capabilities to evaluate the ability of different systems architectures to handle disaster scenarios.

4. Integrate response based on sensors that measure indoor air quality (overlaps with controlling outside air during normal operation).

5. Develop ability to continue operations in the building when no off-site power is available as a result of sabotage or weather-related problems. Demonstrate robustness via modeling, analysis, and prototyping.

6. Develop control of systems to operate only critical systems when on-site generation and distributed generation is limited.

7. Create models to effectively show the potential impacts of on site wind and PV and fuel cell systems and UPS systems on demand and energy rate structures.

8. Develop predictive capability for whole building traffic flow coupled with emergency status of escape routes.

\subsection{Demand Response}

\subsubsection{Background Needs}

Demand-response programs reward customers for reducing their electric demand during periods of electric power emergencies and when the price of electricity is high. However, despite significant public investments in the energy management and control system infrastructure in buildings and facilities, recent studies indicate that most participants used manual load reduction strategies (Neenan et al. 2003; Goldman et al. 2002). This suggests that either the penetration of the enabling technology is relatively low or that the BAS technologies are not adequate for automating load reduction strategies. It is commonly believed that a higher degree of automation is a key ingredient to broaden the participation to smaller commercial customers as well as to improve the demand responsiveness of individual and aggregated loads.

The demand responsiveness of each individual building is highly dependent on occupancy, operational constraints, and the weather. The time dependency of resource availability poses a difficult task for a facility operator to determine how responsive the building can be at any given hour during the season. A building in a demand response program needs load management strategies designed to reliably control the subscribed or committed resources. These strategies require integration of HVAC, lighting, and on-site generation systems to develop comprehensive curtailment strategies that maximize the utilization of all available resources in a building. Advanced and enabling technologies that automate load management strategies are key for higher participation rates. This will reduce the cost for executing load curtailment strategies and will enable participation of smaller commercial buildings, which do not have building operators.

The ultimate demand response program is to allow customers to participate in competitive energy markets. Real-time tariffs are being offered in several utility areas and are expected to become more widespread as liberalization in the electric industry takes hold. Real-time pricing is attractive because it reduces customers' average cost of energy. The downside is that passing price volatility to the end customer is unfavorable if the customer is unable to act upon price signals in an effective manner. For 
that matter, some customers may not even be concerned or wish to be troubled with managing energy costs which may be comparatively low compared with other costs of building operation. Therefore, advanced control and communication technologies are key enablers of customer participation in competitive energy markets. Load curtailment, storage (implicit by cooling/heating building fabric or explicit, e.g., via ice storage), and distributed generation (including combined heat and power) are three main instruments of demand management. Supervisory control of building subsystems provides an extra dimension to achieve more cost effective energy procurement and load shifting. Finally, demand management should be viewed not only as a tool to reduce the overall energy costs and price volatility but also as a tool to "right-size" building systems. It is commonly known that building equipment is sized to design day conditions (extreme day conditions). Integrating sizing with demand management and control strategies eliminates conservative "worst case" assumptions underlying the design day sizing paradigm.

If the curtailment signal is received shortly before the shed is required, all that can be done is to reduce HVAC, lighting and other consumption in such a way that the maximum demand reduction is obtained with the least inconvenience, discomfort etc. If the signal is received the day before, it may be possible to pre-cool the fabric of the building at night and in the morning in order to reduce the overheating discomfort in the afternoon. In each case, there is a need to develop and test control strategies for different types of HVAC and lighting systems. A particular issue is the ease with which zone temperature set points can be raised or lowered. This requires controllers that are connected to a communications network and algorithms that can respond to a global set point change. In order to estimate the total demand response potential for a region or the whole country, it will first be necessary to survey a representative sample of the commercial building stock to determine the relative frequency of types of HVAC and lighting systems and key characteristics of the configurations of the control systems.

\subsubsection{Current Status}

Several demand response programs have been launched throughout the United States in recognition that the demand sectors (industrial, commercial, and residential) can contribute to the electric grid reliability during periods of electric power emergencies and when prices of electricity are high. These programs are administered by vertically integrated utility companies or by independent systems operators, an entity that emerged as a result of competitive electricity markets, with the mandated responsibility to operate regional power grids and to assure safety and reliability. Recently, the New York Independent System Operator (NYISO), the Independent System Operator-New England (ISO-NE), and the operator of the wholesale electricity markets for the Pennsylvania, New Jersey, and Maryland region (PJM), as well as utilities such as BG\&E, KPC\&L, and Xcel offered demand response programs. All demand response programs reward customers for reducing their electric demand during periods of electric power emergencies and when the prices of electricity are high. The requirements for participation and the incentive structures, however, vary widely across the programs.

Several million dollars in public funds have been spent developing and deploying technology to upgrade facilities and prepare commercial and industrial customers to increase their demand responsiveness.

Some states have installed interval meters to the point of saturation, allowing the metering or submetering of facilities and the tracking of load reduction performance. Modern notification technologies have been used to alert facility managers to the need for load reductions during emergency conditions. Connectivity gateways are installed in facilities to enable the aggregation of individual loads by 
integration of energy management and control systems over wide area networks. However, despite significant public investments in the energy management and control system infrastructure in buildings and facilities, recent studies indicate that most participants used manual load reduction strategies (Neenan et al. 2003; Goldman et al. 2002). This suggests that either the penetration of the enabling technology is still relatively low or that the EMCS technologies are still not adequate for automating load reduction strategies. A higher degree of automation within facilities and buildings is a key ingredient to broaden the customer participation to smaller commercial customers as well as to improve the demand responsiveness performance of individual and aggregated loads.

The Federal Energy Regulatory Commission's (FERC) notification of a standard market design (SMD) for competitive electricity markets stated that demand side resources shall be given the opportunity to participate in competitive markets (FERC 2002). The implication of FERC SMD for the demand sector will most likely be that all loads including commercial buildings will be more engaged in the competitive power markets for the benefit of damping high volatility in the electricity markets and to enhance the overall reliability of the power grid.

The impacts of broader participation in demand responsive programs could be significant for electric power system reliability and the overall price of electric services. In the recent NYISO 2002 Emergency Demand Response Program, the $670 \mathrm{MW}$ of load curtailment in a 31-GW power system provided system reliability benefits estimated at $\$ 1.7$ to $\$ 16.9$ million (Neenan et al. 2003).

With greater demand responsiveness, price volatilities in the existing and emerging competitive markets can be dampened. Furthermore, demand responsiveness during high price periods eliminates the use of higher cost and less efficient generator units, which are generally used for supplying power during system peaks. This results in less emission and overall improvement of the efficiencies of the generation mix by utilizing more efficient base load generators.

Key for higher customer participation rates are advanced and enabling technologies that automate load management strategies. These will reduce the cost for executing load curtailment strategies or will enable participation of smaller commercial buildings that do not have building operators.

Currently manual curtailment strategies are only viable during very rare curtailments (1 or 2 per year). As commercial building owners become more engaged in competitive markets, curtailment events may increase dependence on the level of engagement. As the number of curtailment and load management strategy events increases, the building or facility operator is no longer in the position to execute the strategies manually without advanced energy management and control technology.

With the emergence of new market designs, building owners and operators are often overwhelmed with the assessment of the demand response opportunities for their particular buildings. Since each building and facility is unique with respect to its operating schedule, mechanical equipment, and energy management and control infrastructure, it is very difficult to assess the demand responsiveness of each building. Furthermore, the demand responsiveness of each building is not constant. It is highly dependent on occupancy, operational constraints, and the weather. The time-dependency resource availability poses a difficult task for the facility operator to determine how responsive his/her building can be at any given hour during the season. 
In order to broaden the participation of the demand response programs, tools are necessary that guide the facility and buildings owner to assess the responsiveness under a range of load conditions. Furthermore, once a building is in a demand response program, load management strategies need to be designed that can be reliably executed to provide the subscribed or committed resources. These strategies require integration of HVAC, lighting, and on-site generation systems to develop comprehensive curtailment strategies that maximize the use of all available resources in a building.

\subsubsection{Proposed Research}

The R\&D efforts listed below are envisioned to target the following objectives: 1) to broaden the participation in demand responsive programs to include more small commercial customers and 2) to enable the US buildings industry to participate more actively in demand response program. Both objects will enhance the reliability of the electric power supply and dampen price volatility in the power markets.

Develop software tools that facilitate the assessment of demand responsiveness. The tools will support the exploration of opportunities for load curtailment and on-site generation with existing mechanical systems as well as provide guidance on cost-effective investments to improve load management flexibilities.

1. Develop off-line tools that inform the building owner about the risks associated with participation in demand response programs, such as the financial exposure if participants do not perform as agreed. The tools should provide options for assessing risk mitigation strategies.

2. Develop controls technology for executing load curtailment strategies for varying notification periods ranging from near real-time to far-ahead (in the order of days) load curtailment. The curtailment strategies should be comprehensive including: (1) load curtailment of the HVAC system, (2) dimming or turning off lighting, (3) modulating, sequencing, or shutting off vertical transportation, (4) reducing plug loads, and (5) dispatching on-site generation. For instance, if a curtailment signal is received, the controls technology will need to be able to dispatch load curtailment subject to operational constraints.

3. Develop communication standards that support curtailment services such that technology providers can develop new application programs for curtailment services. Analogous to DOE's support of standards work for DG interconnection (IEEE P1547), DOE would be in a good position to support standards activities that would create communication standards for curtailment services.

4. Develop optimal concurrent design of demand management strategies, energy procurement and management strategies, building aggregation, and building system sizing under stochastic (realtime or near real time) energy price models (gas and electricity) using a combination of load curtailment, storage, and distributed generation/combined heat and power.

\subsection{Distributed Generation}

\subsubsection{Background Needs}

Distributed generation systems (DG) that might be deployed in or near buildings in the near future cover a range of technologies including emergency generator sets, PV systems, fuel cells, and microturbine systems, perhaps in combined power and thermal applications.. The key issue is how they can be controlled in both the economic dispatch and electrical senses at the low costs necessary to make adoption attractive. Issues with these technologies are first and foremost, high first costs relative to grid power; interaction with other sub-systems, such as production of hot water; start-up time; stochastic availability, 
aka "clouds" for PV; and maintenance downtimes. The mathematics of economic dispatch on utility scales is well known and is, in fact, one of the most studied of engineering economic problems, but this must be applied at DG scales. Current electrical controls are rudimentary and limited to start and stop instructions and grid synchronization and disconnect. Participation in deregulated markets poses large price volatility risks on the participants in return for lower average prices and more efficient use of available resources. Distributed generation can play a vital role since it allows customers to respond to price signals by switching between grid and locally produced power as pricing conditions change. By doing so, customers will tradeoff risks associated with electric price volatility for risks associated with gas price volatility. The spread between the two prices is key to reduction of overall volatility.

Advances in controls are needed to provide complex dispatch suitable for heterogeneous technologies, combined heat and power (CHP) applications, "island" operation (disconnected from the grid), and operating inertia-less systems. The potential marginal cost advantages of DG require controls to be effective at lowering costs. Some DG systems require long startup and shutdown times making it helpful to forecast demand for electricity and heat in order to determine if the cost of startup is worth the benefits (avoided costs of peaking power). Typically, interconnection rules require fast disconnection in times of outage. The drive for "gourmet" (premium) power comes in response to the deployment of technologies that require it, most notably computing, communications, and control systems themselves. Joint control of end-uses and power sources is required.

\subsubsection{Current Status}

DG vendors are developing control systems and building control systems are being extended to allow modulation of DG systems to meet the building loads. These systems include uninterruptible supplies and grid-interaction issues.

\subsubsection{Proposed Research}

Research is needed to develop strategies and algorithms for distributed generation in the following areas:

1. Mismatch of electrical and thermal constraints, and the effective use of both thermal and electricity storage

2. Design of buildings such that DG technologies match building needs or design of DG systems to better match current building needs

3. Special environmental restrictions that are not common in utility-scale systems must be studied (e.g., noise restrictions for microturbines)

4. Control schemes for DG that allow islanded operation

5. Inertia-less operation

6. Determining and implementing heterogeneous power quality requirements

7. Rapid changes in load (matching loads based on price and resource availability)

8. Balancing demand reduction with reserve/storage for emergencies (PV)

9. Interactions with building energy modeling for predicting future loads (long start-up fuel cells)

10. Load aggregation and management of a fleet or cluster of buildings. 


\subsection{Design of Optimized Systems}

This is the first of two sections on optimizing control applications. This first section focuses on the design of the building system and the strategies used to meet objective functions. The next section will focus on the operational phase of the building. Buildings can be controlled in many different ways and “optimal” control scenarios are assumed to exist whereby some performance index (energy, demand, costs) can be minimized through algorithms and control instructions.

\subsubsection{Background Needs}

Much of the energy savings potential in buildings is in the design of the envelope and the related HVAC and lighting systems. The first goal in optimized systems is to design and implement systems that can be optimized and have flexibility.

It is also important that these systems be easy to understand and be maintainable. "Black-box" solutions are acceptable as long as that part is readily available for replacement. Documentation is helpful for diagnostics. Many optimal solutions require integration of disparate building systems that will tend to increase the already severe complexity and costs of a BAS.

Finally, simply heuristic guidance is needed to help designers implement solutions to complex control strategies. These solutions may be near-optimal, but operators need to know how to operate the equipment they have, otherwise it is often disabled or disconnected.

\subsubsection{Current Status}

"Optimal” control strategies have been the subject of considerable buildings research in some areas (Braun et al. 2001). Many algorithms have been developed relating to the adoption and implementation of new equipment. Such equipment includes ice-storage systems, pre-cooling buildings, heat-recovery wheels, and economizer controls. Some individual buildings have been test subjects for creating integrated algorithms based on operator experience to create "optimal” systems (Pless and Torcellini 2002).

\subsubsection{Proposed Research}

1. Coalesce information for designers of advanced systems providing lists of strategies that work for operating typical equipment. These should be constructed in terms of equipment type.

2. Expand current simulation tools to study the impacts of controls and new energy-saving building systems technologies on buildings. Such technologies could include, but are not limited to, indoor air quality enhancing technologies that can save energy by reducing external ventilation requirements and advanced daylighting concepts that can significantly reduce electrical lighting load. Building system and control simulation tools will aid the design process and the programming of building control systems by specifying the most effective EMCS strategy.

3. Develop hardware schematics to improve the design of systems including equipment selection, valving, and pumping configurations for optimized systems. Examples include coupling heat recovery systems with indirect cooling and process cooling, using free cooling by bypassing the chiller and using tower cooling only, mixing direct evaporative systems with DX systems, and bypassing heat recovery systems for economization. Each of these needs appropriate control strategies to operate the system in a logical fashion. Many times integrated systems are developed, but not operated properly. Analyze and assess integrated building system energy management strategies using whole building models. For example, evaluate the benefits of 
managing lighting according to knowledge of occupancy or using a strategy that modifies the speed of the elevator based on daily energy demand and costs.

4. Analyze a wide variety of buildings, climates, local energy costs using whole building models to derive operational "design rules" that are scalable and adaptable across building types.

\subsection{Optimal Control}

\subsubsection{Background Needs}

Real buildings always operate and perform differently than predicted in the design stage. Sometimes, equipment is "field-engineered" rather than following plans. Also, equipment performance changes with time. To achieve optimal control, tuning is required throughout the lifetime of the building.

It is expensive to maintain building control systems. New computer code must be added whenever there is replacement of equipment, changes in operation schedules, occupant complaints, and improved knowledge of systems that needs to be incorporated. This coding is often done without knowing the total scope of the control system. The objective is often to "make it work," rather than to make it work optimally.

\subsubsection{Current Status}

There have been numerous case studies evaluating real buildings. As a result of the evaluation, control changes are made — often after formal commissioning. Significant energy savings have been realized after the controls modifications. Many times the modifications were made to make the building operate as it was intended - and not as-commissioned. Examples of case-studies that illustrate these issues include Chesapeake Bay Foundation, Zion Visitor Center, and Oberlin College (Pless and Torcellini 2002).

Some equipment has been developed using "fuzzy logic" with the goal of better system control. These types of systems supposedly can learn behavior resulting in better control of the system. Often times, however, they are confusing to operate and do not produce the intended result. The outcome can be frustrating for the user as humans second guess, and override the control systems.

\subsubsection{Proposed Research}

Research is needed to address how to better alter control of systems in real time. Case study research needs to be generalized to apply to broader types of buildings.

1. Individual systems need adaptive controls including variable speed drives, lighting controls, airto-air recovery and economizers. Some work has been done on chillers and larger equipment as well as self-tuning variable speed loops. These systems need to be robust and demonstrate that performance can be improved.

2. Development of performance indices that include time-of-use pricing or other utility charges, comfort, and maintenance. These indices will be used to determine the optimal performance of the building.

3. Development of algorithms that improve current standard algorithms, such as reset control.

4. Development of systems that can learn about the interactions of the HVAC with the building and self-develop algorithms that learn building behavior without additional programming. These systems must also be able to communicate to users how these changing algorithms are affecting system operation. 
5. Development of decision support systems for guiding operators in tuning controls to achieve building operation as intended.

\subsection{Natural/Hybrid Ventilation}

\subsubsection{Background Needs}

Traditionally, building controls for commercial buildings are oriented around a sealed envelope where the design intent is that all outside air enter the building through the air system and the building is to be kept at a slight positive pressure. Natural ventilation refers to passive flow of outdoor air in/out of a building (e.g., open window) for air quality or free cooling. Hybrid ventilation refers to mechanically supplementing airflow when natural flows are insufficient. Natural/hybrid ventilation schemes present new control problems that are complicated because they span many systems that are not self-contained. There is a need for control strategies for advanced natural and hybrid ventilation schemes that maintain indoor air quality and thermal comfort while minimizing energy use. These strategies might use data from sensors ranging from $\mathrm{CO}_{2}$ (and other pollutant sensors), to air velocity, ambient weather, occupancy, window/damper positions, etc. Predictive modeling of such systems requires the ability to simulate buildings with airflow models coupled to energy models that include HVAC systems and advanced controls. A clearer understanding of how such systems can be controlled is needed to overcome the doubts of U.S. building owners, designers, and occupants and to resolve building code issues.

\subsubsection{Current Status}

Buoyed by perceived success in Europe, natural ventilation has become a fashionable subject for research. Dampers and controls from smoke control systems are being adapted for use with natural ventilation. Research on hybrid ventilation is being coordinated through an International Energy Agency (IEA) task group. There are, however, concerns about comfort, dust, and air-distribution, to name a few.

\subsubsection{Proposed Research}

Research into controls issues for natural/hybrid ventilation is needed in the following areas:

1. Operating paradigms for advanced natural and hybrid ventilation systems that apply to U.S. buildings

2. Simulation programs to model coupled thermal, airflow, and controls. The result would be heuristic rules for better control and coupling with HVAC systems.

3. Technical evaluation of actual projects to show reliable control in the real world. Industry wants proof that these systems can be designed and operated such that comfort standards can be maintained. Because of weak modeling in this area coupled with lack of examples, engineers and owners are reluctant to employ these types of systems.

\subsection{Self-Configuring Systems}

\subsubsection{Background Needs}

Today's state-of-the-art in building control systems falls far short of the plug and play expectations that exist for the computer industry. Achieving plug and play capabilities will require standardizing communication protocols (see Section 3) and adding more intelligence to individual components to be able to support self-describing essential features of the devices (see Section 2). Furthermore, automated verification of correct input and output assignments of control devices is needed to enable self- 
commissioning capabilities (see Section 1). As local intelligence is added to sensors and controllers to support plug and play and self-commissioning, there may develop new opportunities to incorporate more sophisticated control algorithms and instructions in a distributed fashion.

\subsubsection{Current Status}

Most sensors and subsystem controllers do not have significant local processing capabilities.

\subsubsection{Proposed Research}

Research is needed to:

1. Develop and evaluate algorithms and instructions that can take advantage of distributed intelligence within control systems

2. Develop intelligent sensors to support plug-and-play applications. 


\subsection{SENSORS AND CONTROLS}

\subsection{Overview}

With the high number of components in large, complex commercial building, it is difficult for operation staff to remain fully aware of equipment and system conditions. Without automated monitoring and fault detection, and the sensors and controls on which they rely, performance can degrade. The number and range of types of sensors installed in commercial buildings today is inadequate to provide sufficient automated (or even visual) monitoring. The primary impediment often cited to more and better sensing is the cost of additional sensors. Installed costs of sensors need to be reduced and decision makers need to become informed regarding the benefits they can derive from better sensing and control.

Automatic controls need to be developed that control indoor conditions adequately so building staff have confidence in control systems. Control based on more plentiful sensors is required to control at the level desired by occupants and to optimize energy use. Optimal control techniques at the system and wholebuilding level are needed to reach the level of performance where high-quality indoor conditions are provided at minimum net energy use. Control must be extended from individual independent loops to system level controls to achieve least-cost, highly efficient, building operation.

Sensor and control needs for commercial buildings span a broad range of technical activities. Sensors at a sufficiently low cost are needed for a broad range of measurements that includes lighting quality, volumetric fluid flow rates, rotational position, wear, vibration, and power consumption, as well as the usual measurements of temperature and humidity that are currently performed in commercial buildings. In addition to possessing lower installed cost than today’s sensors, R\&D must lead to sensors with enhanced performance: longer lives, greater reliability, higher accuracy, persistent calibration. These enhancements will lead to higher, persistent, performance of building systems.

In addition to improving the quality of the sensors themselves, streamlined installation is required. One of the largest cost components for sensors is the cost of installation. Installation, particularly in retrofits, requires running cabling in spaces such as walls and ceilings that are frequently difficult to access, running up expenses for labor.

\subsection{Scope and Organization}

A generic monitoring and control system is shown in Figure 4.1. This system can be divided into four generic component systems: sensors, controls, actuators, and other software. The first three categories are included in this chapter.

Besides sensor elements themselves, the sensor category includes signal conditioners and (wired and wireless) communication hardware and software. Controls are devices for converting measurement signals from sensors into (electrical or pneumatic) signals sent to actuators that implement the corresponding action on a physical device or system component. The controls category includes both control software and hardware and the underlying principles.

Actuators are the devices that convert signals into physical action. A control system requires each of these three types of components. The fourth category, which includes performance and condition 
monitors, automated fault detection and diagnostic (FDD) tools, and other software, is covered in the final chapter.

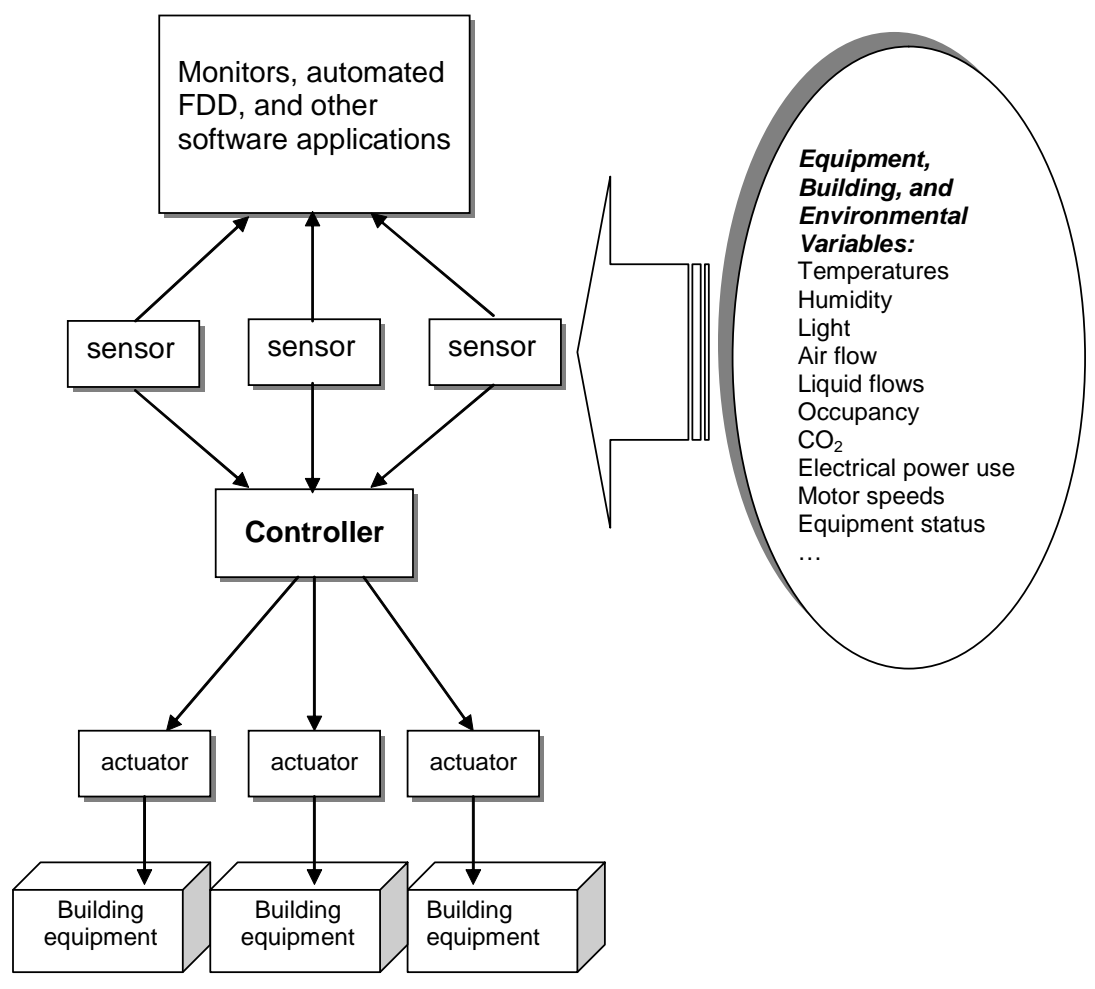

Figure 4.1. Simplified conceptual diagram of generic monitoring and control system

This section is organized into three major parts, each corresponding to a major component category in a generic control system as identified in the previous section. Each of these is further divided into subsections, each devoted to a separate technical aspect of that category. A fourth major section is included, which captures topics and issues that cut across the three categories based on control-system components.

\subsection{Advanced Sensors}

\subsubsection{Background Needs}

Any advanced control strategy for increasing occupant comfort, decreasing energy consumption, and ensuring safety in buildings relies on accurate and reliable sensors. New technologies may help to ensure the accuracy of sensors through improved sensing methods and intelligence built into the sensor for self diagnostics. Emerging technologies will facilitate broader applications of sensors in buildings including automated diagnostics of HVAC, lighting, fire and safety systems, demand-responsiveness and optimal control, as well as indoor-air quality monitoring and counter measures against bio/chem attacks.

These new monitoring and control applications will demand new sensor development as well as improving existing sensors to drastically reduce the cost. As more sensors are deployed, methods for networking the vast system of sensors will be needed to make the best use of the sensor data. 
Interoperability and self-identification will be important as the number of sensors in buildings increases. Some of these sensors will be wireless while wired sensors may remain an alternative option. In either case, efficient schemes for powering the instruments must be developed. As sensors are deployed more extensively in buildings, new applications will emerge, and research will be needed to incorporate new sensors for aspects of building performance that may not typically be thought to impact building control systems.

\subsubsection{Current Status}

Sensors are currently prevalent in several building control applications. One of the most important types of sensor for monitoring energy efficiency and for fault detection is an electrical power/current meter. New solid-state meters allow for accurate measurement of this fundamental quantity.

For determining the optimal performance of HVAC equipment, the most established environmental sensors are those for measuring temperature, relative humidity (RH), carbon monoxide (CO), and carbon dioxide $\left(\mathrm{CO}_{2}\right)$. To measure temperature, both mechanical (e.g., thermally expanding metallic coils) and electrical means (e.g., thermistors, metallic RTDs, thermocouples, digital P-n junctions, infrared thermocouples) provide sufficient accuracy for current needs and have proven to be fairly reliable. The most common RH sensors in HVAC systems yield an output that is proportional to either the capacitance or resistance of a hygroscopic material.

The accuracy of these sensors, however, is often questioned, largely because of the significant drift that occurs after initial calibration. Sensors for monitoring $\mathrm{CO}_{2}$ concentration have been used in limited applications for demand-controlled ventilation. The most popular sensors contain a small cell in which infrared light is passed through the air sample. The absorption of the infrared light can be related to the $\mathrm{CO}_{2}$ concentration. These sensors are currently more expensive than other sensors used for building applications.

One other constituent that is monitored for input into ventilation control systems is carbon monoxide. Carbon monoxide concentrations can be used to determine necessary ventilation rates in structures where fuel-burning equipment resides such as garages. As evidenced by the performance of CO sensors in homes, however, false alarms have been a source of concern. Existing equipment for detecting other constituents of interest for air quality, including volatile organic compounds (VOCs) and particles, is both large and expensive and therefore not currently suitable for continuous monitoring.

For controlling variable air volume distribution systems, sensors that measure the airflow in ducts are available, but their performance is not as reliable as needed. Air velocity can be measured by wellestablished techniques such as Pitot tubes, but individual sensors often do not sufficiently capture the average flow rate through the system owing to the non-uniform flow that is present in the ducts.

Occupancy sensors are valuable tools for energy conservation that are used extensively to turn off lighting and other equipment when no occupants are present. Infrared detection of people or ultrasonic detection of movement have been the predominant means of achieving such detection. Additional lighting control can be achieved by monitoring the amount of daylight so that lighting levels can be suitably adjusted. These sensors may also have a role in HVAC controls if a need exists for real-time determination of solar heat gains. 
Most commercially available sensors are designed to report back to a central monitoring location.

Processing and memory abilities have not been incorporated in many of the sensors that are currently used in buildings.

\subsubsection{Proposed Research}

Various sensor technologies are being developed in laboratories, many of which may yield techniques to better monitor conditions in buildings. For most building applications, it will be imperative that these sensors achieve a low per-unit cost because multiple installations are necessary. Future R\&D efforts on advanced sensors fall into several categories.

Improvements to existing sensors and features of new sensors that should be investigated include:

1. self-calibrating, self-testing, self-diagnosing, and self-reporting sensors

2. low-cost power meters for sub-metering

3. low-drift and low-cost RH sensors

4. sensors that automatically detect the need for a measurement

5. peripatetic sensors that automatically provide measurements from mobile objects or people and that can automatically be detected by sensor networks

6. incorporation of low-cost processing and memory on sensor elements to generate information from raw data and to store that information, reporting data only when anomalies occur

7. inexpensive sensors for short-term monitoring.

The following new types of sensors are needed for building applications:

1. volumetric airflow sensors

2. affordable VOC and particulate sensors

3. mold sensors

4. sensors for homeland security issues (chemical, biological, and nuclear hazards). Identification of important hazards is necessary and the development of flexible methods of deploying these sensors will be important. Currently, these sensors are cost prohibitive for widespread deployment, especially considering the large number of potential hazards (the relation to energy usage will need to be explored).

5. low-cost sensors that detect occupancy. HVAC and lighting controls could be adjusted based on knowledge of position of occupancy, resulting in improved comfort and lower energy costs.

6. inexpensive sensors for IAQ assessment.

\subsection{Novel Sensors}

\subsubsection{Background Needs}

Typical applications of sensors in buildings are meant to modulate the performance of the HVAC system for thermal comfort, adjust lighting levels, or ensure the safety of occupants. Recently, however, more constituents are being monitored for fault diagnostics, humidity control, and improved air quality, while various pieces of building equipment contain sensors that monitor their performance. In addition to the sensors meant for continuous monitoring of buildings, a wide array of sensors for short-term monitoring 
such as temperature probes, power meters, humidity probes, and mobile chemical analyzers are available to practitioners. Sensors for other aspects of buildings exist that may not appear to be directly related to energy use and thermal comfort but may find use in the future. For example, strain sensors are widely used to monitor structural safety and could be applicable for widespread building monitoring.

New technology for special applications may enable investigators to examine building components in greater depth than is possible with sensors that are permanently placed in a building. For example, some sensors may only be needed shortly after construction, such as monitoring equipment for VOC emissions from carpet or furniture or determination of moisture levels arising from building materials that have been sitting on a wet jobsite. Other sensors developed for short-term use could aid in the commissioning of buildings by verifying the operation of HVAC systems or for in-situ calibrations of control instruments. Alternative continuous monitoring applications may also emerge. Real-time load monitoring by determination of heat losses and gains from the structure could enhance the efficiency of HVAC systems and the comfort of occupants. Incorporation of schemes to communicate with existing equipment and appliances will allow better monitoring of energy usage and could allow for peak-load shaving by turning appliances off during certain periods of the day. Distributed generation from energy sources such as photovoltaics or fuel cells may require improved power sensors for integrating local power production into the power grid. Finally, structural monitoring could greatly increase the safety of occupants and could also have an impact on energy consumption. Structural sensors may help indicate incidences of envelope or framing failure that allow for escape of heat from the building environment or infiltration of unwanted chemicals.

\subsubsection{Current Status}

The main challenge in this area is to define the role of the various sensors for building monitoring. Additionally, in the case of sensors attached to equipment, the ability to interface with other building sensors will be important. Sensors used for short-term monitoring may not necessarily suffer from the same cost constraints as sensors meant for widespread deployment. If used repeatedly over the life-cycle of several buildings, higher cost sensors could be viable given sufficient opportunity for operators to recoup their investments. For emergency situations, cost may not be a severe constraint either. For these situations, reliability, ease of use, and accuracy will be the predominant characteristics for acceptance in the marketplace.

\subsubsection{Proposed Research}

The following list presents proposed future R\&D efforts related to special applications of sensors:

1. methods of integrating data from sensors that are integral to equipment into the building control system

2. development of structural sensors that can detect breaches in the thermal envelope or duct systems

3. development of sensors for detecting occupant hazards from structural failure due to earthquakes, mudslides, floods, wind, decay, pests, or other natural phenomena

4. development of sensors to determine occupancy and population distribution within buildings

5. development of flexible sensor platforms for short-term monitoring 
6. determination of the suitability of existing weather sensors for real-time load calculations, solar and wind energy generation monitoring, and structural safety monitoring

7. instrumentation to accurately determine power generated by alternative energy sources such as photovoltaic arrays and fuel cells for proper control of distributed energy systems connected to the power grid.

\subsection{Powering Sensors and Controls}

\subsubsection{Background Needs}

Reducing the energy requirements of sensors will make it possible to power the components of sensor and control networks from very small energy sources or from "scavenged power" (electricity captured or generated from the ambient environment). Some of the small energy sources that are presently under development will likely last for long periods of time (10 to 20 years perhaps). These include betavoltaic batteries, helium-ion sources, and small hydrogen-production fuel cells. Future methods for scavenging energy from the environment might include photovoltaic, thermoelectric, and vibration-to-electric technologies, as well as inductively coupling energy from the commercial building's power system. The combination of reduced energy requirements and diversification of energy supply will allow sensors to be placed in more locations within a new building and providing more options for installing sensors in existing buildings.

\subsubsection{Current Status}

Most sensor systems require energy to conduct measurements, acquire data, and transmit it to data collection systems. Along with the reduction in the size of sensors come reduced energy (power) requirements. The current state of power source technology is typically based on either available AC power or conventional battery power. Batteries with energy density adequate to last for a considerable period of time are typically quite large as compared to the size of small sensors (e.g., micro-sensors or nano-sensors).

\subsubsection{Proposed Research}

1. Investigate on-going alternative battery and fuel cell developments for insertion into sensor networks within commercial buildings. These developments will likely require additional engineering to make them suitable for the intended environment.

2. Develop power-efficient scavenging and harvesting technologies that can be engineered in such a way as to effectively operate a sensor network. The techniques will essentially lead to the netzero energy operation of sensor systems.

3. Develop power management techniques for the sensor system that will efficiently conserve its energy use.

\subsection{Sensor System Testing and Qualifying}

\subsubsection{Background Needs}

In addition to ensuring that sensors and controls operate properly when configured into a system subject to common environmental variables, today systems must continue to function when subjected to vandalism, sabotage, and even terrorist acts. Today's methods for testing and qualifying sensor systems do not extend into this realm. This indicates a need for a systematic process that extends into testing 
performance subject to these sorts of stressors. An Operational Test and Evaluation Program would help ensure that vendor equipment performs as intended and would make explicit its limits of operability.

A testing and qualification program would help ensure that sensor systems will operate and function as expected in field use. An Operational Test and Evaluation (OT\&E) Program could serve this function. The OT\&E program's goal would be to ensure that systems are successfully developed and qualified before being deployed for actual field use. Through the application of consensus-based standards (International Engineering Consortium, Military, and ANSI) and by subjecting the systems to the actual environmental conditions they will experience, system risks and susceptibilities could be identified. A strategy can then be developed and implemented to eliminate or mitigate these issues prior to the delivery of systems to the user community.

The OT\&E Program would include testing for environmental effects on sensor system performance and functionality. The types of effects include temperature (steady-state and transient), humidity, vibration and shock, contaminants (such as gases, vapors, and fine particulates), electromagnetic compatibility and interference, lightning induced transients, power supply variations, and ambient pressure. The system performance would be monitored during each of these environmental tests.

A relatively new set of "environmental” conditions would include security issues such as vandalism, sabotage, and terrorist acts. These conditions would need to be quantified and consensus-based standards developed and validated. Once new standards were established, the resulting tests could be added to the OT\&E to enhance the reliability and functionality of building sensor systems. It may be necessary to characterize these environmental conditions in actual buildings to ensure the testing covers actual field/operational conditions.

The OT\&E Program would provide systematic and comparable testing, employ and develop testing protocols that are standards based, and help to identify environmental vulnerabilities and operational limitations. The vulnerabilities and limitations can then be addressed by the manufacturers and retested to validate the corrections and improvements. This systematic approach allows for comparable results that help ensure equipment and systems will meet their application requirements in field use.

\subsubsection{Current Status}

Not applicable. No testing and qualification program like the one described in this section currently exists.

\subsubsection{Proposed Research}

Research and development activities would include the following:

1. identification and characterization of environmental conditions to which sensor systems are subjected

2. development of testing procedures and standards for operational testing and evaluation. 


\subsection{Advanced Controls}

\subsubsection{Background Need}

Controls are the devices and algorithms that take data from sensing and data acquisition systems and convert it to desired actions and signals to instigate action by actuators for controllable devices, such as dampers, valves, on/off switches, and other devices whose states or modes of operation can be varied. Most controls in HVAC systems today take three general forms: 1) scheduling and mode selection, 2) direct digital proportional, integral, derivative (PID) control, and 3) pneumatic control.

Mode selection is usually done on a schedule based on time of day or day of year. For example, airhandling fans in an office building may be scheduled for certain hours when occupants are expected to be present, say 5 a.m. to 10 p.m. on weekdays, and off at all other times. In some cases, more advanced control selection of modes may be used, for example, air-handling fans might be turned on based on data from occupancy sensors.

Direct digital PID control is commonly used in modern building systems to control devices whose load changes over shorter periods of time than a day. These devices can be on/off controlled, like thermostats in homes, or modulated, such as a chilled-water valve in some air-handling units. PID control is linear by definition, even though many devices to which it is applied in buildings are non-linear. In most cases, during normal operation, this does not present a problem because the non-linear devices and loops can be considered linear over ranges of operation.

Pneumatic control was used for many years in commercial buildings to control modulating devices. Over the last 20 years or so, direct digital control has gained favor for new installations, but many buildings still control devices, especially terminal units, using pneumatic control.

Advanced controls are needed to improve mode selection by basing it on anticipated conditions (feedforward and predictive control), to optimize control at the multi-loop, system, and whole-building levels, to retrofit existing control systems (e.g., pneumatically controlled terminal boxes) with interfaces for monitoring and integration with building automation systems and for integrating systems (such as heating, cooling, and power) to more efficiently utilize our energy resources while providing better indoor environmental conditions. The subsections that follow present the case for research in advanced controls organized into four categories: advanced controls, retrofitting controls, controls behavior, and special considerations.

Much of current control technology relies on synchronous point-to-point communication, which does not work well with controls distributed over a wide area. New control technologies that use packet-based communication are needed. These new technologies will tolerate delays and will be much more robust; in addition, they will support plug-and-play and self-configuring devices.

Deregulation of the utility industry provides both challenges and opportunity for the commercial building sector. In a deregulated environment buildings have to be demand responsive because the reserve margins will be significantly less than the margins in the regulated environment. Making buildings and their systems more demand responsive will require advanced control technologies that go beyond the current state of the art. These new technologies will allow building systems to directly interact with utilities and the grid. Direct interaction will permit retail customers to respond to dynamic pricing, which 
will lead to improved economic efficiency and reliability, and reduce the need for new generation capacity. Furthermore, it will mitigate price volatility and provide much needed discipline to the wholesale markets.

Finally, because there is a shortage of well-trained and motivated operators there is a need to make controls autonomous and fault tolerant. For example, if a failure is detected, controls should be reconfigured where possible and in the worst case they should make sure that the performance of the system degrades gradually rather than abruptly.

\subsubsection{Current Status}

Building control has come a long way from simple bi-metallic room thermostats to sophisticated microprocessor-based distributed digital controllers. Yet many buildings with building automation systems are poorly operated and maintained. Furthermore, only 7\% of commercial buildings have central controls. Development of low-cost advanced control technologies can provide tremendous opportunity to improve operation efficiency in buildings.

Over the past four decades, the advent of analog and digital electronics has allowed control technology to spread far beyond its initial applications (Murray et al. 2003). ${ }^{1}$ Much of the pioneering research and development efforts in controls has come from universities and other industries, such as aerospace, manufacturing, industrial, and processing industries.

Although over the past two decades many advanced controls concepts such as adaptive, nonlinear, geometric, hybrid, fuzzy, and neural control have been theorized, developed and in some case implemented in other industries, the building control industry is yet to embrace these concepts. For much of the last two decades, the building control industry worked on developing standard protocols [BACnet ${ }^{\circledR}$ (ANSI/ASHRAE 2001) and LonWorks ${ }^{\circledR}$ (http://www.lonworks.com)]. More recently many building control manufacturers are working on integrating building controls with the Internet.

\subsubsection{Proposed Research}

Following are the proposed research activities in the advanced controls area:

1. Adaptive and Predictive Controls: With utility deregulation and widespread use of distributed energy resources (micro-turbines, fuel cells, and diesel generators), there is a need to develop adaptive and predictive control algorithms. These new control algorithms will make use of the forecasts (load of the building, energy price, and weather) and energy databases to make autonomous decisions on whether to generate power locally or to buy the power from the grid. In addition, they will also significantly improve the plant efficiency by optimizing the equipment and resource utilization. This work can be accomplished in the near term.

2. Personalized Ultra-Local Controls: Traditional approaches to creating a comfortable and productive working environment do not make the entire building comfortable. It is difficult to satisfy the needs of all occupants with the same environmental settings. New control technologies should be developed so that BAS can provide customized and personalized comfort for every occupant in the buildings. Technologies should consider the job content as well as personal preferences for light levels, temperature, and humidity. This technology will not only

\footnotetext{
1 The entire panel report, titled "Control in an Information Rich World," can be accessed at the following URL http://www.cds.caltech.edu/ murray/cdspanel/report/cdspanel-15aug02.pdf.
} 
improve the delivery efficiency but also may translate to significant productivity improvement. This work can be accomplished in the mid to long term.

3. Autonomous Fault Tolerant Controls: Challenges in traditional control installation are configuring, tuning, and commissioning the controllers, sensors, and actuators. This process is not only error-prone and time consuming, but also requires a skilled technician or an engineer. Development of controls based on an open distributed computing architecture with plug and play devices can overcome many of the shortcomings of the traditional building controls. Developing autonomous fault-tolerant control technologies and the necessary infrastructure will provide significant productivity benefits and improve operation efficiency in the commercial building sector. This work is a long-term research and development activity.

4. Demonstrations to validate and verify advanced controls performance.

5. Development of advanced controls using whole building models in a rapid prototyping environment. (i.e., model predictive controls using hardware-in-the-loop techniques.)

6. Integrated building controls. Development of an energy management system that takes into account fluctuations in prices, loads and weather forecasts, connected to a network that informs occupants and trims usage according to peak demand.

7. Controls that automate HVAC commissioning and rebalancing of airflows periodically or as a result of a building renovation.

8. Fault tolerant, supervisory building controls for managing energy generation with demand. For example, supervisory controls for generators connected to energy storage devices. Demonstration of a supervisory control strategy and the energy savings.

9. Controls that optimize HVAC system performance to achieve IEQ and energy savings.

\subsection{Retrofitting Controls}

\subsubsection{Background Need}

Control retrofits fit into three broad categories: 1) control system replacement, 2) partial retrofits of control systems to provide improvements to control, and 3) retrofits to provide services not previously the focus of building controls. The first of these, control system replacement, involves completely removing existing controls and replacing them with an entirely new control system. Installation of a site-wide building automation system (BAS) to provide all control functions digitally falls into this category. Because the entire system is replaced, we will not consider this a control retrofit but a new installation, and therefore, it will not be included in this section. The second category, partial retrofits, includes all installation of controls equipment and systems in which new or replacement equipment is added to an existing control system. This includes replacement of individual controllers, replacement of control algorithms with new ones, installation of replacement or new HVAC equipment with new on-board controls, and integrating legacy pneumatic controls with direct digitals controls, among others. The third category captures installation of new controls or control algorithms to meet new needs. This includes retrofitting HVAC controls to provide mitigation of terrorist threats or to improve life safety in emergencies. These applications represent new functions for HVAC and other building control systems, but they require integration with traditional control functions.

The major R\&D challenges for control retrofits are 
- increasing the compatibility of control devices to achieve true plug and play across controls from many different vendors

- developing low-cost, yet effective, controls retrofits for common problems, such as interfacing pneumatically controlled equipment with digital BASs

- increasing the flexibility of control systems to meet new, even currently unanticipated, needs of the future

- developing safety and security retrofits for existing controls and equipment to provide security for the public at reasonable cost.

\subsubsection{Current Status}

Standards, such as BACnet (ANSI/ASHRAE Standard 135-2001) have simplified the job of retrofitting direct digital controls somewhat. Proprietary standards, whether closed or open, are still common in the market, so difficulties persist today regarding compatibility of controllers and integrating replacement devices into BASs. Pneumatic and digital systems have not been integrated well. Many (maybe most) buildings with pneumatically controlled terminal boxes as well as a BAS cannot monitor or control those terminal boxes via the BAS. Because a large commercial building may have hundreds of terminal boxes, this leaves the condition of a significant amount of equipment unknowns and receiving service only when significant failures have occurred, causing occupant complaints or during a major remodel of the space served by a set of boxes. These situations present opportunities to save energy and improve indoor conditions through improved operation and maintenance.

\subsubsection{Proposed Research}

Proposed research topics are as follows:

1. retrofitting controls for homeland security - protecting building occupants from potential threats to ventilation systems and other intrusion

2. plug and play for retrofit controllers

3. developing retrofit interfaces between pneumatically controlled subsystems and DDC. An example is locally pneumatically controlled terminal units that operators want to monitor from a central control room

4. research into increasing the flexibility of control systems to support new (even unanticipated) needs

5. developing low-cost wireless technology to reduce installation costs associated with control retrofits.

\subsection{Sensor-Control Interaction}

\subsubsection{Background Need}

Improved characterization of the sensitivity of controllers to sensor performance is needed. Existing building emulators can play a role in evaluating controller hardware, while computer simulations of controller performance may allow designers to gauge the controller response to sensor failures.

Computer emulation of building conditions that are fed into controllers will speed the adoption of new technologies by providing a resource for testing controller hardware under a complete range of conditions. Such simulations will enable designers to determine problems before the controllers are 
installed in actual buildings. Similarly, computer simulations of the controller with inputs from actual sensors could help determine the sensitivity of the controller to various sensor outputs. Among the concerns for controller operation is the performance when sensors do not provide accurate signals or fail altogether. Determining the sensitivity of controls will give sensor manufacturers an accuracy target and will provide better statistical probabilities of the energy consumption in buildings.

\subsubsection{Current Status}

Tools currently exist for simulating controls in a virtual sense. Controllers can be interfaced with sensor outputs from computer simulations of building performance to judge their performance in a variety of situations.

\subsubsection{Proposed Research}

1. Evaluation of the sensitivity of controls to sensor failure, drift, and inaccuracy

2. Simulations of controllers and control systems

3. Building emulations in which real sensors and controls are used and evaluated.

\subsection{Sensor \& Control System Integration}

\subsubsection{Background Need}

The utilization of sensor data for multiple purposes (fire, security, energy) will ultimately help reduce cost to the building owner. In the near-term, cost savings are associated with operational improvements by utilizing data that otherwise would not have been available. Using the example mentioned above, an occupancy sensor installed for a lighting control system may also be used as an input for determining the heating or cooling demands of that particular zone in a building.

As the sensor technology advances, we expect more product offerings of multi-sensor devices that can be networked into the wired or wireless building automation systems. This trend is likely to support and perhaps expedite the integration efforts that we already observe in the building automation industry. Systems integration is likely to lead to operational improvements by sharing data across all buildings functions and by maximizing the informational content of measured and collected data.

As sensor and control networks become more integrated, adequate data security and safeguarding measures must be developed and applied to prevent access violations and intrusions by unauthorized personnel. Data access and firewall protection methods need to be developed to limit access to control actions, alarm notification, and system-critical information dissemination.

\subsubsection{Current Status}

In today's building control systems, each sensor takes measurements and transmits this data to the control system to which it is connected. The utilization of sensor data across multiple control systems is not common, primarily because today's sensors are highly specialized single-use sensors, which may not measure data that are relevant for other control systems. In applications where sensor data could be shared, stringent code requirements limit or even prevent cross-utilization of sensor data. Fire alarm systems must meet rigid sensor monitoring requirements. Therefore, sensors sending data to a fire control panel must possess the necessary monitoring capabilities required by the national and local fire codes. However, sensor data collected for a fire alarm system could be shared with control systems with less 
rigid requirements. For instance, an occupancy sensor installed for a fire alarm or life-safety system could be used for controlling lighting and HVAC as well.

\subsubsection{Proposed Research}

1. Conduct research to quantify benefits of building controls systems integration and identify technological gaps that would support greater integration.

2. Conduct research that characterizes the vulnerability of integrated wired and wireless sensor and control networks in buildings.

\subsection{Actuators}

\subsubsection{Background Need}

Actuators are the portion of a control system that can control or vary the output of a physical device or process, such as a relay, variable damper, motor, or lighting ballast. Actuators are usually closely coupled or directly attached to the equipment they control, thus the electrical integration of actuator to physical equipment is usually straightforward. On the other hand, inexpensive actuators usually do not embed intelligence; intelligence usually resides in the controller, which is often separated from the actuators by a considerable distance.

Two technologies are having an impact on actuators for building controls - power line carriers and wireless communication.

\subsubsection{Power Line Carriers}

Power line carrier (PLC) systems use electronic wiring devices to send information via a high-frequency signal over the $120-\mathrm{V}$ and $277-\mathrm{V}$ electrical power distribution systems of a building. For example, PLC systems are used in automatic clock systems (master time systems) to synchronize all of the clocks in a building or to reset the time after a power outage. In a PLC system, a generator is used to impose a 1- to $4-\mathrm{V}$ high-frequency signal on top of the existing voltage sine wave. This signal is generally in the 2500to $9500-\mathrm{Hz}$ range, with some older systems operating at $19,500 \mathrm{~Hz}$ or higher. The advantage of PLC is that control signals are transmitted from controller to actuators over the in-place wiring. Thus PLC eliminates the need to run separate control wiring, which is a very expensive proposition in existing buildings. The problem with PLC is that some building equipment can "absorb" the injected PLC signal preventing proper operation. For example, some electronic ballasts that are capacitive can absorb the signal from a PLC system. As a result, the signal becomes too weak to be "heard” by the receiver (like a time clock) connected to the power line.

\subsubsection{RF Communication}

The most widely used wireless standards are IEEE $802.11 \mathrm{~b}$ and Bluetooth. ${ }^{\circledR}$ Table 4.1 summarizes the key technical characteristics of the two systems (Industrial Wireless Technology for the $21^{\text {st }}$ Century, December 2002). 
Table 4.1. Characteristics of Two Wireless Communication Standards

\begin{tabular}{|l|l|l|}
\hline & \multicolumn{1}{|c|}{ IEEE 802.11b } & \multicolumn{1}{c|}{ Bluetooth } \\
\hline Effective Distance & 500 meters & 10 meters \\
\hline Spread Spectrum & Direct sequence (DHSS) & Frequency hopping (FHSS) \\
\hline Data Rate & $11 \mathrm{Mbps}$ & $721 \mathrm{kbps}$ \\
\hline
\end{tabular}

Given the typical distances between actuators and controllers in most building control applications, it is unlikely that short-range Bluetooth will play a major role in the operation of actuators. On the other hand, IEEE 802.11b (or a subsequent faster wireless standard) will likely be the winning wireless approach for actuators if the price point per actuator is considerably reduced. Because there are usually many more actuators for lighting ballasts than for HVAC system components (such as dampers), wireless will likely impact HVAC systems first since cost pressures are less severe. The key challenges facing the application of wireless communications for actuators are 1) vulnerability to interference, 2) security, and 3) power management.

The power management issues facing wireless actuators are less daunting than those facing wireless sensors. Actuators, by their nature, are connected to the electrical equipment they control. Since most equipment usually has a source of electrical power even when the equipment is off, this electrical power can be used to power the connected actuators. Since power may not always be present, some actuators will still require batteries.

\subsubsection{Current State of Technology}

In the analog mode of operation, actuators are generally directly wired to the physical system controlled. For example, the actuator that controls the position of an HVAC damper is usually integrated into the damper linkage. The control signal that dictates the specific state of the actuator (open, closed, etc.) is usually transmitted from a physically remote controller using low-voltage wiring. In conventional control systems, many actuators are operated by one controller output. This requires that wiring be run between every actuator and controller - a process that is prone to errors and is prohibitively expensive in existing buildings.

In direct digital control (DDC) systems, which are increasingly common for HVAC applications and are beginning to be considered for lighting systems (for example DALI - Digital Addressable Lighting Interface), actuators are digitally operated, often from remote field panels that contain a controller as well as digital connections to other field panels. Digital actuators can be operated from simple field busses (multiple actuators connected to a single pair of wires and controlled digitally), which reduces the wiring problems of analog actuators.

\subsubsection{Proposed Research}

1. $R \& D$ to harden wireless actuators from outside interference.

2. $R \& D$ to increase the security of wireless actuators so they cannot be operated or interfered with by unauthorized users.

3. $\quad R \& D$ to reduce the power requirements of wireless sensors and actuators

4. Develop intelligent sensors/actuators that have functionalities of self-calibration, validation and correction, and alarm and diagnosis. Traditionally, actuators such as expansion valves are only used as devices for flow control. Development of smart actuators allows additional functions such 
as valve health monitoring, flow rate metering, and valve operating curve correction and calibration (linearization), and closed loop control.

5. Study the benefit of migrating closed loop control functions into the actuators, which also have certain capabilities for process fault diagnosis.

6. Study the benefits of integrating intelligent sensors and actuators.

7. Develop affordable and scalable network architecture of smart actuators/sensors.

8. Optimization of diagnostic and control functions to be built into the intelligent sensors and actuators.

\subsection{Communication Modes: Wired vs. Wireless}

\subsubsection{Background Need}

The key promise and opportunity of wireless technology in building operation is to significantly reduce the cost of installing control systems by eliminating the control wires. Installation of wiring can represent $20 \%$ to $80 \%$ of the cost of a control point in HVAC or lighting control networks (Kintner-Meyer et al. 2002). The availability of low-cost wireless sensor and control systems could not only reduce installation costs overall, but also lead to increased use of sensors and control devices necessary to establish and maintain highly energy-efficient building operations and productive and healthy work spaces.

Only 5.4\% of the existing U.S. commercial buildings (23.5\% of the U.S. commercial floor area) are equipped with an energy management and control system (EMCS) (Katipamula and Gaines 2003). They tend to be large buildings with a mean floor area of about 50,000 $\mathrm{ft}^{2}$. The remaining commercial building stock typically uses thermostats to control air temperature in the building zones with little special resolution for heating, cooling, or ventilation requirements, resulting in climate conditions that are often too hot or too cold or insufficiently ventilated. Large buildings with EMCS are typically sparsely equipped with sensors in order to limit the overall capital cost of the control system. They too lack data on indoor climate and lighting conditions with sufficient spatial resolution to perform optimal control, automated diagnostics, and energy efficiency monitoring. This lack of detailed operational information limits the ability to control and diagnose HVAC, lighting, and life and safety systems, resulting in suboptimal buildings operation.

Other key features of wireless sensors and controls are shown in Table 4.2.

Table 4.2. Features of Wireless Sensors and Controls

\begin{tabular}{|l|l|}
\hline \multicolumn{1}{|c|}{ Features } & \multicolumn{1}{c|}{ Benefits } \\
\hline No wiring & Avoidance of wiring cost significantly reduces the installation cost. \\
\hline Improved flexibility & $\begin{array}{l}\text { Not necessary to locate sensors near wires. Wireless sensors can be easily } \\
\text { relocated as the indoor space undergoes re-configuration. For instance, a } \\
\text { wireless light switch can move with the interior wall. }\end{array}$ \\
\hline Ease of servicing & $\begin{array}{l}\text { Wireless sensors can be easily removed from their location for re- } \\
\text { calibration or replacement in case of failure. }\end{array}$ \\
\hline $\begin{array}{l}\text { Extendability of existing } \\
\text { wireless network }\end{array}$ & $\begin{array}{l}\text { Once a wireless network is established, additional sensors and controllers } \\
\text { can be easily added at the cost of an additional wireless device. }\end{array}$ \\
\hline
\end{tabular}

Wireless transmission of buildings operation data will be key to drastically reduce the installation cost of sensors and control products. If cost can be significantly reduced, more sensors are likely to be deployed, 
which will enable new control, monitoring, and diagnostics applications in commercial buildings, including

- Optimal controls strategies to minimize the cost of energy used in commercial buildings.

- Demand responsiveness that would enable the building to reduce electric demand during periods of grid emergency or high electricity prices with no or minimal impacts to the occupants.

- Automated diagnostics that would test the proper performance of HVAC systems to maintain high energy efficiency performance.

- Indoor air quality monitoring to maintain healthy and productive work environments.

- Effective counter-measures for biological and chemical attacks on buildings.

\subsubsection{Current Status}

Major advancements in wireless sensors and control technology have been made in the industrial processing and manufacturing industries with some spill-over effect to building automation industry. Early adopters of wireless technologies are Johnson Controls, Siemens, and Delta Controls offering wireless temperature sensors that are either fully integrated into wired building control networks or provide non-networked direct communication between devices. Wireless lighting switches are offered by Lutron Electronics Co. (Lutron 2003) and others.

Most of the wireless sensor and control products commercially available are communicating in the industrial, scientific, medial (ISM) band, that Federal Communications Commission (FCC) set aside for license-free, low-power radio transmission over short to medium distances. The license-free bands frequently used for wireless sensors and controls applications are 300 megahertz (MHz), $433 \mathrm{MHz}, 900$ $\mathrm{MHz}$, and 2.4 gigahertz $(\mathrm{GHz})$.

\subsubsection{Proposed Research}

Wireless sensors are relatively new to the buildings automation industry. Confidence needs to be gained that wireless sensors and controls will work reliably and perform as designed. To find acceptability in the building automation industry, the following challenges must be faced:

- Proven operational reliability: wireless sensors need to gain industry confidence by demonstrating reliability in commercial buildings.

- Invulnerability to interference: as more and more wireless devices are being used in commercial buildings there is a need to demonstrate that the wireless technologies are not vulnerable to interference by other wireless systems, cellular telephones, and other devices that emit in the electromagnetic spectrum.

- Power requirements: There is a need to store more power capacity on wireless sensors to maintain reliable operations for five years or more. Novel power management concepts and power salvaging methods need to be developed to ensure proper operation.

- Integration into existing networks. Today's wireless technology is mostly designed to work in a stand-alone mode. Future wireless networks need to be easily integrated into existing wired control networks.

- Interoperability. As wireless sensors and control devices become more common there will be a need to develop specifications and standards that assure interoperability. 
The following R\&D agenda is proposed:

1. Develop wireless sensors with a targeted cost reduction of a factor of 10 compared to today's wired sensors by 2006.

2. Develop a wireless controller for VAV retrofit applications in existing buildings.

3. Develop wireless sensor and control devices that use power scavenging schemes for self-powering or battery lifetime extension.

4. Develop open architecture schemes and interoperability specifications that promote market competition of technology developers.

5. Develop self-configuration procedures for wireless devices for automatic set-up and transmissionpath finding.

6. Develop strategies for auto-commissioning using wireless devices that can be distributed around the building, are automatically detected by a network, and provide feedback for tuning.

7. Develop strategies for localized fault detection by wireless devices or automatic network maintenance checks. 


\subsection{Networking, Security, AND Protocols AND StandardS}

\subsection{Overview}

Before the benefits of advanced controls can be realized, a supporting infrastructure must be in place. Networks, protocol standards, and security are the infrastructure elements that enable advanced controls. The advanced building automation and control (BAC) system requires network hardware and protocols that support communication within the building between devices and controllers as well as communications out of the building to remote sites, utilities, and other service providers.

Beyond the physical networking hardware and devices, there is a need for security, both of the physical building and network equipment as well as of communications off site and potentially within the building itself. Some of that network security is implemented in devices such as firewalls that are operated by the corporate IT department and some of the security is built into the protocols themselves. Open protocols also provide a robust medium for communication, allowing interoperability between equipment of different vendors, expansion to accommodate new sensor development, and new connections to industries that will need to tie into the building control system (BCS).

We have some idea of what kinds of technology we want to enable: demand response, distributed generation, better control (finer grained, distributed, smarter), interoperable devices, wireless sensors, web-enabled applications, connections to service providers (utility, emergency responders, energy services, performance monitoring, security), self-configuring networks and devices, automated diagnostics and commissioning, systems that respond to a variety of situations (e.g., fire), common interfaces, etc. Each of these technologies has requirements for networking, security, and standards. It is the goal of this chapter to set forth a plan for defining and understanding these requirements.

\subsection{Scope and Organization}

There are several "big issues" in this chapter. The first issue is the complexity of a control network that now extends outside of the building — as we open the building to outside control (to enable distributed power generation and load control) we now have potential for destabilizing the power grid, and we don't yet have a theoretical understanding of such a system and how to ensure a stable grid. This falls under the general "big issue" of complexity. We see similar problems in communication and security as we link multiple systems together-how do we ensure interoperability and seamless security? Research is needed simply to identify the problems, develop theoretical foundations, and set the course for healthy application and standards development.

Another "big issue" is security itself. We need secure BCS protocols. We need security for inter-building as well as intra-building communications, across the LAN as well as the Internet. We need increased physical security - access control and authorization. This includes the effective integration of biometrics into an intelligent access control system that can process physical access as well as network access. And we need to address secure communication across multiple layers of the communication stack and across multiple networks that may be operating at different security levels. 
And then there is the "big issue" of standards to enable secure, reliable, inter-business communication over the internet and standards for message formats - between the utility and the BCS, and between the BCS and other service partners. These issues are discussed in the following sections.

\subsection{Complex Controls - Building and Grid Interactions}

\subsubsection{Background Need}

In the regulated environment, the interface between buildings and the utility grid has been well understood, because customers only deal with a single local utility that provides all their required power at predictable fixed prices. Local utilities own the generation and the transmission grid, maintain reserve power to assure demand can virtually always be met, and know their customers. In a deregulated environment, economic efficiency improvements will lead to lowering of the reserve margins to significantly less than the margins observed in a regulated environment, potentially causing severe shortterm imbalances in supply and demand with high price volatility. In addition, because of population growth in the urban environments, the transmission and distribution system capacity will be near full capacity.

One response to this scenario is to make buildings more "demand responsive" and allow buildings to directly interact with the grid and other buildings on the grid. The current control technology, although adequate to control systems within the building, is not suitable because it relies on synchronous point-topoint communication, which does not work well at a scale of millions of control points with controls distributed over a wide area. Because of the complexity associated with the sheer scale of the control problem and the ever-changing topology of the system, as buildings choose to respond one day and not the other, and controls that cross the building boundary, advanced complex control technologies are needed that use flexible packet-based communications and innovative control algorithms.

Direct interaction between buildings, and between buildings and the grid, will permit customers to respond to dynamic pricing, improve economic efficiency and reliability, and reduce or defer the need for major capital expenditures for new generation capacity. Furthermore, it will mitigate price volatility and provide much needed discipline to the wholesale markets. In the long term, these technologies will allow better management of distributed local generation of electric power and thermal energy resources that are keys to zero energy buildings and homes. Distributed and local generation of electric power and thermal energy will also enhance the reliability of the grid by alleviating transmission and distribution bottlenecks.

Complex controls technology will allow buildings to use additional information as a precondition to achieving higher levels of efficiency and reliability, whether economic or thermodynamic. What is also true, but perhaps less clear, is that this is a fundamental transformation of our current control system, from a known model to an unknown model of higher complexity (Chassin 2003).

Traditionally, systems have been partitioned in a way that eliminates the need to consider global performance questions, thereby eliminating the efficiency and performance enhancement opportunities the same global perspective provides. The building meter and the distribution feeder are regarded as inviolate regulatory, design, business, legal, and operational boundaries. While these fixed points in the systems have done a great deal to simplify and thereby better enable robust system design and operation, 
we also recognize that we have missed opportunities to realize many efficiency and performance improvements because they are present.

As we open up to the outside, we are increasing the scale of building control from local to global, and along with increasing scale comes increased difficulty in establishing coherent, verifiable, and achievable performance goals. The ability of local (e.g., building, equipment) control systems to respond to and contribute to evolving global (e.g., power grid-wide) situations and objectives is essential to maintaining the overall stability and reliability of the systems (e.g., economic and electrodynamic).

Consider for example the $60-\mathrm{Hz}$ frequency of the electricity at any electrical outlet in a building. This frequency is rarely exactly $60 \mathrm{~Hz}$, rather it varies slightly around $60 \mathrm{~Hz}$, depending principally (although not exclusively) on the balance of electric generation and load. It has long been recognized that frequency is an extremely accurate measure of the balance between loads such as those in buildings and supply at generating plants. More significantly it is ubiquitous - the frequency is absolutely the same in every building on any given grid. Therefore, if frequency were used to modulate loads in buildings through use of technologies that were "friendly" to the grid, then each building or building system could provide a small but valuable service to the grid (Kintner-Meyer et al. 2003). Taken in sufficient numbers, and because dropping load can be done much faster than increasing generation output, many buildings might exert more accurate and more reliable control over the frequency of the electric grid than generators can.

The problem is that the interface between the building's control network and the grid's control network does not permit that value to be metered, and therefore any value there cannot be recovered. So the achievable economic value of such a strategy in today's system is zero. Although the actionable signal (frequency) is available at every point where action can be turned to value (profit), no infrastructure exists by which value can be realized, so the incentive does not exist.

Even if such mechanisms for measuring the global performance properties of very large-scale systems existed, and profiting from the minute contributions of a large number of local and autonomous subsystems to achieve a global performance objective were possible, we do not fully understand what is required to ensure that such systems do not spin wildly out of control. Getting at this understanding is at the crux of a field of study generically called "complexity theory."

So it is that we come to wonder whether increasing the complexity of our building control systems is necessarily tantamount to making more connections, allowing more data, and providing unfettered access to more information. Drawing on the lessons from other systems, we might ask if simplicity does not demand providing fewer of just the critical connections, less of only the right data, and very limited access to the most important information. It is reasonable to believe that, before we open the control of buildings to the influence of outside information, we recognize that the actions taken within the building in turn influence events outside, and therefore we must understand exactly how to manage the complexity that inevitably ensues. Thus we must strive to include building controls and indeed equipment control in the discourse on complexity and vice versa. We must understand what information is needed in what form to achieve the desired performance. We must understand what topology is minimally necessary for stability to be ensured. And finally we must understand how information can be authenticated rather than restricted, because ultimately in these types of systems it is not free access to information that is risky, rather it is dissemination of inauthentic information that is dangerous. 


\subsubsection{Current Status}

Not much work has been done to understand the requirements for development of complex control technology as it relates to energy use and response to the grid. Early results from this work have led to development of an advanced power distribution system simulator that is capable of modeling the interaction between controls within buildings and local power markets at the distribution system level. Efforts are currently under way to extend this capability to support bulk power markets (Guttromson et al. 2003). In addition advanced mathematical models for network analysis are being developed to enable analysis of system stability and dynamic behavior of joint economic and physical control responses (Oliveira et al. 2003).

Much of the work on understanding complex systems comes from other disciplines. In order to understand complex systems, we need to better understand networks. Networks are just about everywhere. Our entire world is composed of natural and man-made networks of varying complexity. In recent decades we have performed more and more research into how the connectivity of individual participants in a network affects the network and vice-verse. Diseases, the brain, river systems, social networks, ecosystems, global markets, and of course telecommunications systems are all dynamic systems of highly interconnected discrete systems whose behavior cannot be understood without considering the network.

However, a number of significant research challenges remain that we will discuss in the next section. It is important to note that many of the results obtained from other disciplines are applicable to building controls and networks, albeit only in a general sense. The principles and mathematical models used to understand emergence and network systems have been shown to be quite general, in spite of the incredible differences that exist in the domains in which they are applied. This is in itself an indication of the convergence of thought that is taking place in the area of complex networks and of which buildings are clearly a part.

\subsubsection{Proposed Research}

1. Develop first-principles model of complex controls. In the short-term (FY05 through FY07), focus on the development of the first-principles models and the transformation of our understanding of networked systems and network-based control. This research will develop the building blocks or models for design of complex systems (buildings interacting with the grid and buildings interacting with other buildings), which allow local control of building systems as well as global coordination between buildings and the grid leading to both economic and thermodynamic efficiency.

2. Develop new control paradigms. In the mid-term (FY06 through FY09), focus on the development of control strategies that make use of the new insights. Control concepts such as contract networks, in which control action is determined through negotiated trades (Smith 1980) and market-based (dynamic-pricing) control systems are examples of new control paradigms that employ the network as the principal performance feedback mechanism. Use of these very powerful strategies today is encumbered by the extreme difficulty of maintaining order and robustness in the systems they govern. Once we understand the emergence of order and we gain a more general concept of robustness in networked systems, we are far more likely to successfully implement control systems that employ these strategies.

3. Demonstrate new control paradigms. Before the new control paradigms are fully implemented in mass, we need to test them in a simulated environment and then demonstrate 
them in the field. This research activity should be undertaken after significant progress has been made on the development of the new control paradigms (FY08 and FY09). This activity will address a number of unanswered questions regarding the specifics of a building-tonetwork linkage.

4. Develop information requirements, standards, protocols, and security. After general strategies for complex control of systems have been developed, there will be a need to determine the quality, quantity, format, transport, and accessibility of the data required.

5. Investigate use of intra and inter building micro-grids. Investigate how micro-grids can be applied to improve efficiency and reduce building system dependency on national grids.

6. Develop robust protocols that allow interaction with the grid (including real time pricing schemes) without compromising building system operation.

7. Develop smart algorithms that optimize load management between power grid consumption and local energy generation while maintaining power grid stability.

8. Develop an infrastructure that will allow building systems to maintain information flow to and from the grid even in the event of a brown-out.

9. Develop controls that allow a building to sense the power demand on the grid but remain insensitive against power outages.

\subsection{Building Control Networks}

\subsubsection{Background Need}

As more companies become aware of the ease of accessibility, they are creating new business models to take advantage of the inherent value of BCS data. Research is required to identify users of this new technology and their associated use cases. As IP connected BCS move beyond their infancy, the effectiveness and adoption of future designs will be greatly dependant on the user requirements of the systems.

BCS over IP technology enables innovative strategies such as:

- remote load shedding

- pre-curtailment warning

- remote load monitoring

- remote performance monitoring

- remote real time energy use intensity (EUI) forecasting

- remote advanced real-time energy modeling

- remote diagnostics

- alarm response

- remote equipment failure alarming

- pre-failure / out or specification alarm

- comfort and indoor air quality (IAQ) assurance

- $\quad$ security and life safety alarming. 


\subsubsection{Current Status}

Historically, building control systems have been isolated, not only from outside the building but also from the building's own IT network. A typical building control system today consists of many distributed single-purpose control sub-systems that coordinate the operation of appropriate loads. Each of these subsystems consists of a controller (with an embedded microcontroller), and as many attached actuators and sensors as are necessary to control the particular class of load. For example, an HVAC system might consist of a central Energy Management System (EMS) and as many distributed field panels as are necessary to control the actuators that are connected to the different mechanical systems throughout the building. These field panels are connected to each other and the central EMS by means of a physical cable (often twisted pair but sometimes Ethernet). The particular protocol used to exchange information between field panels is often proprietary to that manufacturer although the use of BACnet (and other “open” protocols, notably LON) is starting to change this situation. An example of a BACnet-based HVAC control system network is shown in Figure 5.1.

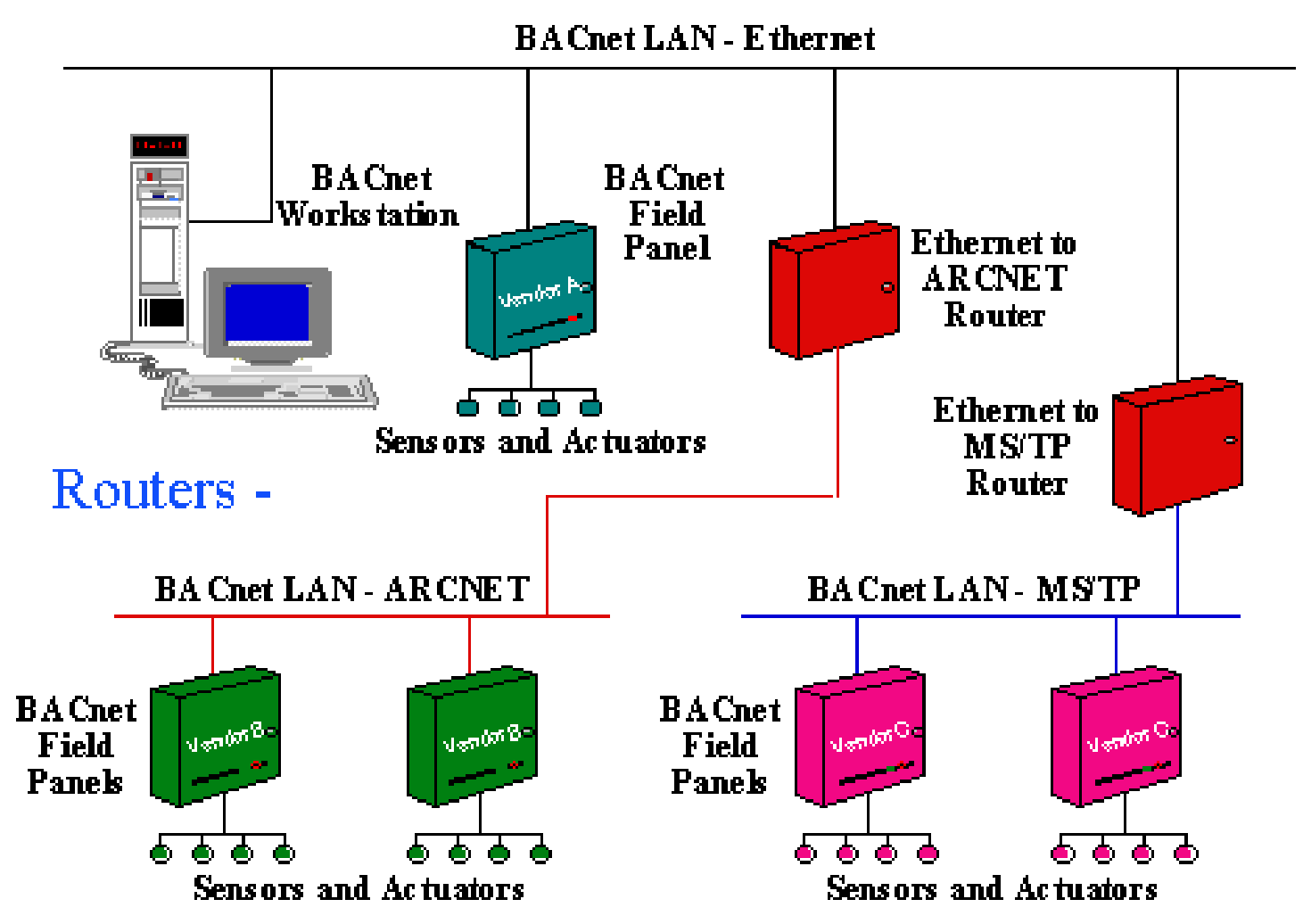

Figure 5.1. Networking architecture for a modern HVAC system using BACnet as the basis for communication between EMS systems.

Lighting control systems are generally less evolved than HVAC systems in terms of their networking potential, as well as the degree to which digital control has played a role in control system design. A modern lighting control system might consist of many individual, independent control systems. Each control system may control loads (groups of lights) at the room level or for larger zones. As with HVAC, 
each of these independent control systems consists of a controller and connected actuators and sensors. Hard wiring carrying analog signals is used to make point-to-point connections between sensors and controllers and between controllers and actuators (relays or possibly dimmers). Some more advanced lighting control systems have adopted digital control techniques to allow data transfer between controllers (again through twisted pair) but these are not in widespread use and are less common than digital communications between HVAC field panels (as above).

Figure 5.2 shows a block diagram for a modern lighting control system designed to implement daylight responsive lighting control and occupancy detection. Note that one actuator (typically a relay) is responsible for switching the high-voltage wiring that connects all the ceiling lighting fixtures in the room or space. The relay operation is, in turn, controlled by the controller, which can sense occupancy using an attached occupancy detector (sensor). Dimming control, as would be required for most daylighting applications, usually requires separate low-voltage control wiring to control the dim level for all the connected ballasts. The controller determines what dimming level the dimmer actuator will call for based on input from the connected light sensor. As indicated in the diagram, the need for both high voltage and low voltage cabling introduces numerous complexities in the design, specification, installation, and commissioning of advanced lighting control systems.

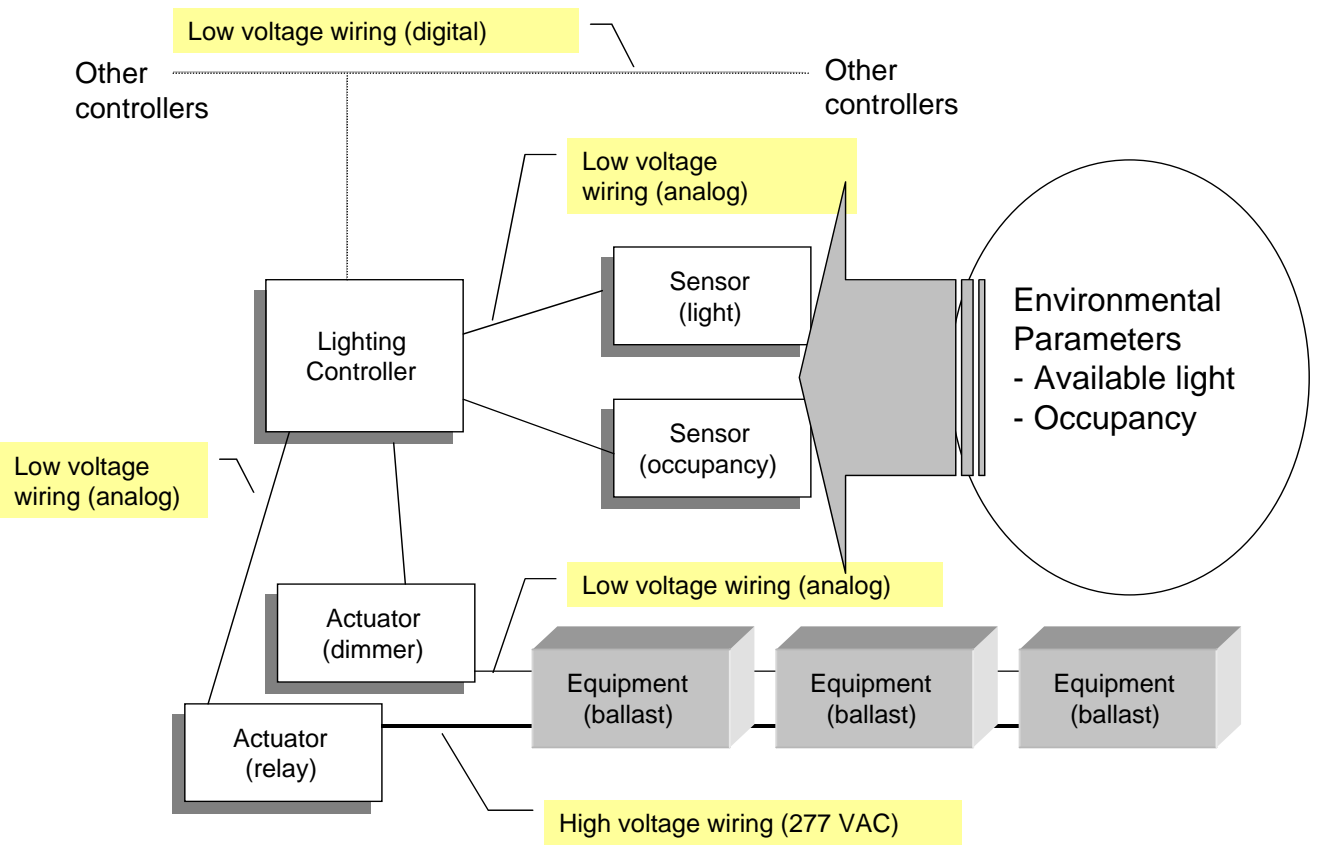

Figure 5.2. Block diagram for a modern lighting control system showing the different system components and the electrical connections between them

This is the present networking configuration for the two elements of the BCS that consume the greatest energy - HVAC and lighting; i.e., largely independent systems with independent control and physical cabling for carrying the control data and information between controllers. This cabling is usually in addition to whatever IT network is already in place to handle the enterprise's IT. It is inefficient to run 
separate physical cables to support the building control systems if Ethernet cabling is already present in the building IT network. Part of this inefficiency is simply historical. Many building control systems were designed before the PC revolution, and were therefore not designed to take advantage of networking technology. In addition, there are institutional barriers to sharing the IT network with building control systems. Although the amount of data traffic added by building control systems may be insignificant to the amount of traffic on a typical IT network, many IT managers are loath to add any traffic onto their IT networks. Given the newness of networked building controls, these concerns should not be trivialized.

From this present situation we see several networking development trends. First, despite the concerns, the IT backbone within networked buildings is increasingly used to support BCS traffic: within buildings (e.g., higher level control communication), between buildings on a WAN (e.g., a campus setting), and between buildings with segments of the internet between (i.e., from one BCS to another). Beyond this it is certain that BCS-to-Service Provider connections will become commonplace in the near future. This first trend might be called "BCS over IP.” A second trend might be labeled new networking architectures: networking for security, and networking to accommodate distributed control with more and smarter endnodes (sensors, actuators) which then creates new opportunities for smarter control (e.g., sharing sensors among different BCS components like HVAC and lights and access control) and new technologies like automatic fault detection and diagnostics (AFDD). These new technologies put greater demand on the network - requiring consideration of response time, bandwidth, etc.

\subsubsection{BCS over IP}

As discussed above, traditional Building Control System(s) (BCS) have been used to control and monitor building systems (HVAC, lighting, etc.) within the confines of the building. The system hardware, wiring, and User Interface access points ended at the boundaries of the building structure. To connect multiple buildings together on a campus, dedicated wires would be run between buildings specifically for the BCS.

With the advent of IP networks within and between the vast majority of commercial buildings, there are new opportunities for BCS. Many BCS manufacturers are now producing devices that communicate with each other over existing IP networks to reduce cost and increase the functionality of their BCS.

These new BCS over IP systems offer the potential to greatly increase energy efficiency, comfort, and safety while reducing maintenance costs. In order to achieve these benefits, there is a strong need for definition and standardization. The purpose of this section is to provide an overview of this technology and identify areas in greatest need of research in order for these systems to become widely adopted.

Existing LAN/WAN/Internet infrastructure can be used to enable

- Building Control System (BCS) devices to communicate with each other. Allows sharing of BCS data (e.g., outside air temperature) between buildings.

- BCS devices to communicate with system operators both on-site and remotely via traditional Human Machine Interfaces (HMI), standard web browsers, internet enabled cell phones, pagers, and others means.

- BCS devices to communicate with Enterprise business systems such as utility billing systems or Enterprise Resource Planning (ERP) systems. 
By leveraging the existing investment of the IP network infrastructure, the BCS is enhanced and extended at a marginal cost.

In traditional BCS, building operators are typically the only users. One or more PC workstations are loaded with expensive HMI software. These workstations, which are usually located in the facility engineer's office, are used for building system monitoring and adjustment of schedules, setpoints etc. Sharing of BCS data with other internal or external parties is difficult and is rarely done.

Once BCS are connected to existing LANs, WANs, and the Internet, the opportunity to make BCS data available to a whole host of new users becomes apparent. These users may include:

- utilities

- $\quad$ building operators/property managers

- $\quad$ energy service companies (ESCOs)

- energy aggregators

- maintenance/service providers

- $\quad$ security providers.

From the traditional independent BCS network (including even dedicated BCS wires between buildings) there is now increasing use of the IT backbone for BCS communication. Many BCS vendors now offer devices that can communicate over an IP network. A possible network configuration that shares the IT backbone is shown in Figure 5.3. This configuration can apply to a shared IP data network within a single building where each branch represents a floor and each BCS sub-branch a subnet on the corporate LAN, or each branch could represent a building in a campus and each BCS sub-branch a BCS network within a building. The "BFR" of Figure 5.3 represents a BCS-specific firewall and router. The router segments the network to confine lower-level BCS communication to the local BCS network or subnet.

The real challenge in networking now is the connection across the internet out to the box in Figure 5.3 labeled "Foreign BACnet/IP (a buildings systems communication standard) device, external network, or external service provider." Some vendors are presently implementing BCS human machine interfaces [the control graphical user interface (GUI)] on web-servers (browser based) to allow monitoring and perhaps control of a facility from outside a building. If "outside" extends over the internet, then security becomes a big concern. Present implementations use VPN technology to connect to remote sites or operate with no security (unless the BCS is sitting behind a facility's IT firewall). Security will be addressed in more detail in the Security section. 


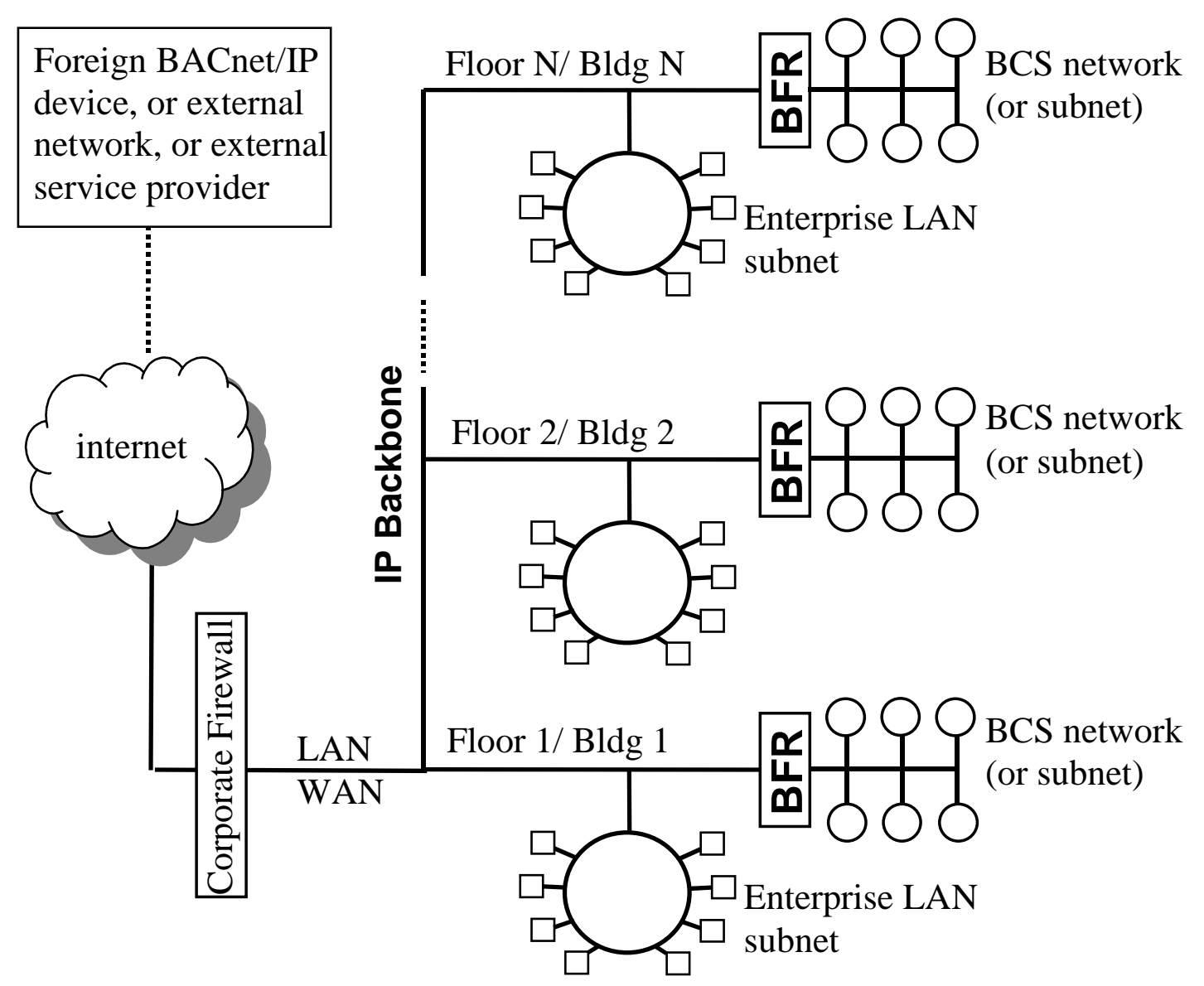

Figure 5.3. Building Network on IT Backbone

The other big issue comes when the BCS is connected to an external service provider-and that is the issue of communication standards (also to be discussed more in the Protocols and Standards section). The service provider does not speak BACnet or any other BCS protocol, so now there is a need for agreement on message contents and format and how to transport and translate the message. Now we need web standards to communicate between gateways, web browsers and business applications (e.g. HTTP, HTTPS, HTML, XML, XML schemas, SOAP, Web services) ${ }^{1}$. This in turn requires representatives from different service industries to meet with BCS community representatives to agree on new standards. This is necessary and will happen-it is the enabler for the envisioned BCS-grid connection that will allow for distributed power generation and load control. And beyond utility connections, there are many other potential service providers as listed earlier. The real potential for BCS over IP is yet to come and the real savings in energy use will largely be tied to the BCS-to-service provider connection.

\subsubsection{New Networking Architectures}

The big picture in networking is the potential for opening the building to the outside (see the last subsection), the power of smarter networks, the possibility for sharing that smarts among the BCS sub-

\footnotetext{
${ }^{1}$ Hyper Text Transfer Protocol (HTTP), Hyper Text Transfer Protocol (Secure), Hyper Text Markup Language (HTML), eXtensible Markup Language (XML).
} 
systems, and the challenge of doing all that securely (next section). Here we look at the potential for smarter BCS networks in the building. As computer intelligence, memory, and networking hardware prices continue to fall, the intelligence of a network can be more and more distributed. This, in turn, allows for lower-level controllers to take more responsibility for control. Even individual devices can be made smart enough to monitor themselves. More sensors allow for smarter use of energy.

What potential applications do these smarter networks allow? If a device can monitor itself, then we can have Automatic Fault Detection and Diagnostics (AFDD). In this scenario, a vendor gives a device (like an air handling unit) some rules to apply to device data (e.g., damper position and frequency of openings and closings, and temperature swings, etc.) to determine if the device components are performing acceptably. This has huge energy saving potential. Work is ongoing in addressing this issue.

Another application is that of sharing sensor data. If we have an occupancy sensor attached to the lighting system, this intelligence allows for smarter lighting control and thus energy savings. But there is also potential for sharing that information with the HVAC system to control room temperature and air exchange rate, for example, when no one is in a room. That information could also be shared with the access control system to identify intruders. And in an emergency, that information could help firefighters to get people out of a building. We are only at the forefront of linking BCS sub-systems in this way and much work remains to be done.

While it is a goal to tie systems together and enable sharing of sensors, there are also competing network issues. Is it cost effective to make every sensor addressable by higher level (central cross-system) controllers? The higher level controllers likely are speaking BACnet/IP over the IT backbone. But little sensors don't have or need the brains to implement the IP protocol or even the relatively simple BACnet protocol. And what about physically connecting a myriad of sensors - the labor of stringing cable, configuring databases, the strain on the network of thousands of little sensors talking and consuming bandwidth?

Even if building control evolves to the point where the control systems use the same physical wires as the IT network, it is generally not cost-effective to push IP down to the very smallest individual loads (such as individual lights that might consume only 50 to 100 watts each). The severity of this situation depends on the nature of the equipment controlled and its power rating. For example, while it is becoming costeffective to exchange TCP/IP (or BACnet/IP which is UDP/IP (User Datagram Protocol over Internet Protocol) based data between HVAC field panels (each of which might control several $\mathrm{kW}$ of electric load), it is not (yet) cost effective to control individual lights or other low-power end nodes in this way. The reason for this is (ultimately) cost and the number of end nodes involved. Implementing the TCP/IP (Transmission Communication Protocol over Internet Protocol) stack (or BACnet stack to a lesser degree) requires a relatively large amount of memory and a capable microprocessor at each end node. This is an expensive proposition when applied to many small end nodes (such as lighting).

Although it is too expensive to implement the TCP/IP stack at numerous small end nodes, at least at the present time, there are many sensor bus systems available today that represent cheaper alternative networking architectures. Many of these sensor buses (such as Profibus and Modbus) were designed originally for industrial process control. However, the basic networking techniques are appropriate for low-cost building equipment control networks. 
One example of a low-cost equipment network that can be applied to the problem of small end node control in buildings is the IBECS (Integrated Building Environmental Communications System) network under development at LBNL to control the operation of building lighting control systems.

IBECS is a practical networking system that takes advantage of a building's existing IT infrastructure to control lighting components and other building equipment through the Internet. To achieve this, IBECS applies recent developments in hardware and software known as embedded device networks. New manufacturing techniques can produce semiconductor devices that incorporate an integrated circuit, unique IP address, and simple LAN communications at a very low cost per control point or end node. These slave devices are "embedded" into building equipment such as lighting ballasts, allowing these components to communicate digitally over a simple network or bus. The IBECS project applies Dallas Semiconductor's 1-Wire embedded device network to building lighting equipment control. In the 1-Wire system, embedded devices are connected to the 1-Wire ${ }^{\mathrm{TM}}$ network (also called the microLAN) and an Internet-connected master controller (or bridge). The master initiates all communications between embedded devices using the 1-Wire protocol and can transmit and receive information from an authorized PC or server. A diagram of the IBECS concept is shown in Figure 5.4.

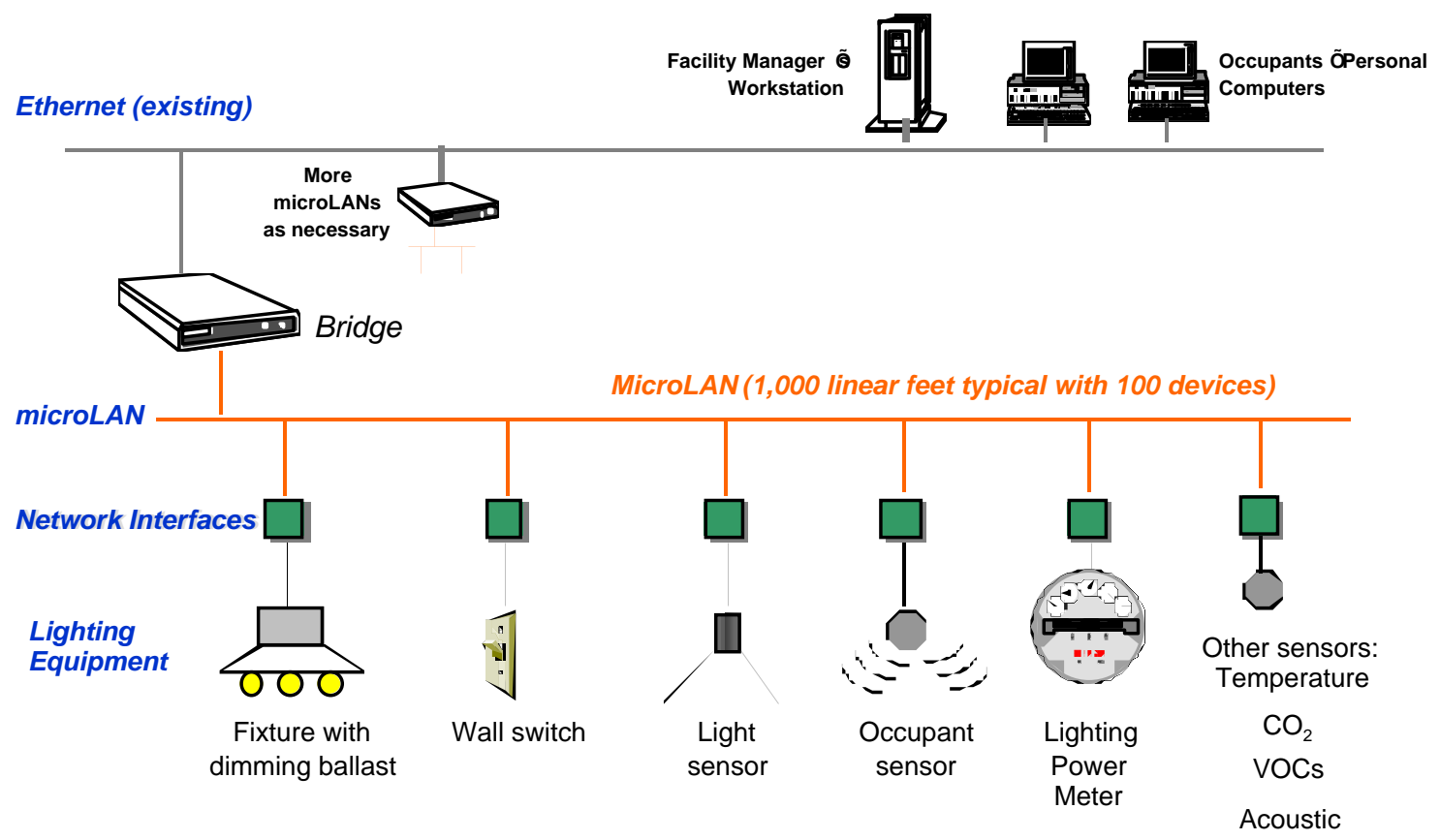

Figure 5.4. The IBECS network architecture. The microLAN bridge intermediates data flow between the facility's Ethernet and the microLANs that control and communicate with individual building loads.

It is assumed that IBECS will be installed in a building that already has a TCP/IP network for the facility's computer LAN (local area network). IBECS piggybacks onto the enterprise's IT network, using bridges to connect the facility's computer LAN to smaller sub-networks, or microLANs. Each microLAN is a minimalist digital network (low speed, minimal number of conductors) that physically interconnects all the lighting and other loads within one physical zone. The MicroLAN bridge controls and monitors lighting equipment attached to each microLAN and transfers collected control and status information over 
the existing TCP/IP network to client browsers or databases. The bridge controls multiple equipment loads on one side and accepts and transmits data back to the TCP/IP side. This architecture allows control of many small sub-networks that are directly connected to the load interfaces they control.

The master-slave networking architecture used by IBECS is similar to that of other low-cost control networks (for example, the MS-TP master slave token passing shown in Figure 5.1. Master-slave networking architectures have significant security advantages over a peer-to-peer networking architecture. In peer-to-peer operation, each connected device has equal "status" to other devices and each device can initiate network communications independently. This is in contrast to master-slave architectures where only the master device can initiate network communications. Since only the master is directly connected to the Internet, only this component must be hardened to attack from the outside. The many slave devices attached to each master cannot be directly addressed from the Internet at all, which reduces their vulnerability to attack.

What we have with IBECS then is a lower-level segment of the BCS network. BACnet (see section on Protocols and Standards for more details) is used as the protocol for most (or all) of the HVAC system, but it is not economical to extend BACnet (much less TCP/IP) to the numerous lighting ballasts and lighting sensors. Instead we implement a simpler network and a gateway/bridge to pass sensor information up to the BACnet network and take commands from the BACnet network. An example of how the IBECS system could co-exist with BACnet is shown below in Figure 5.5.

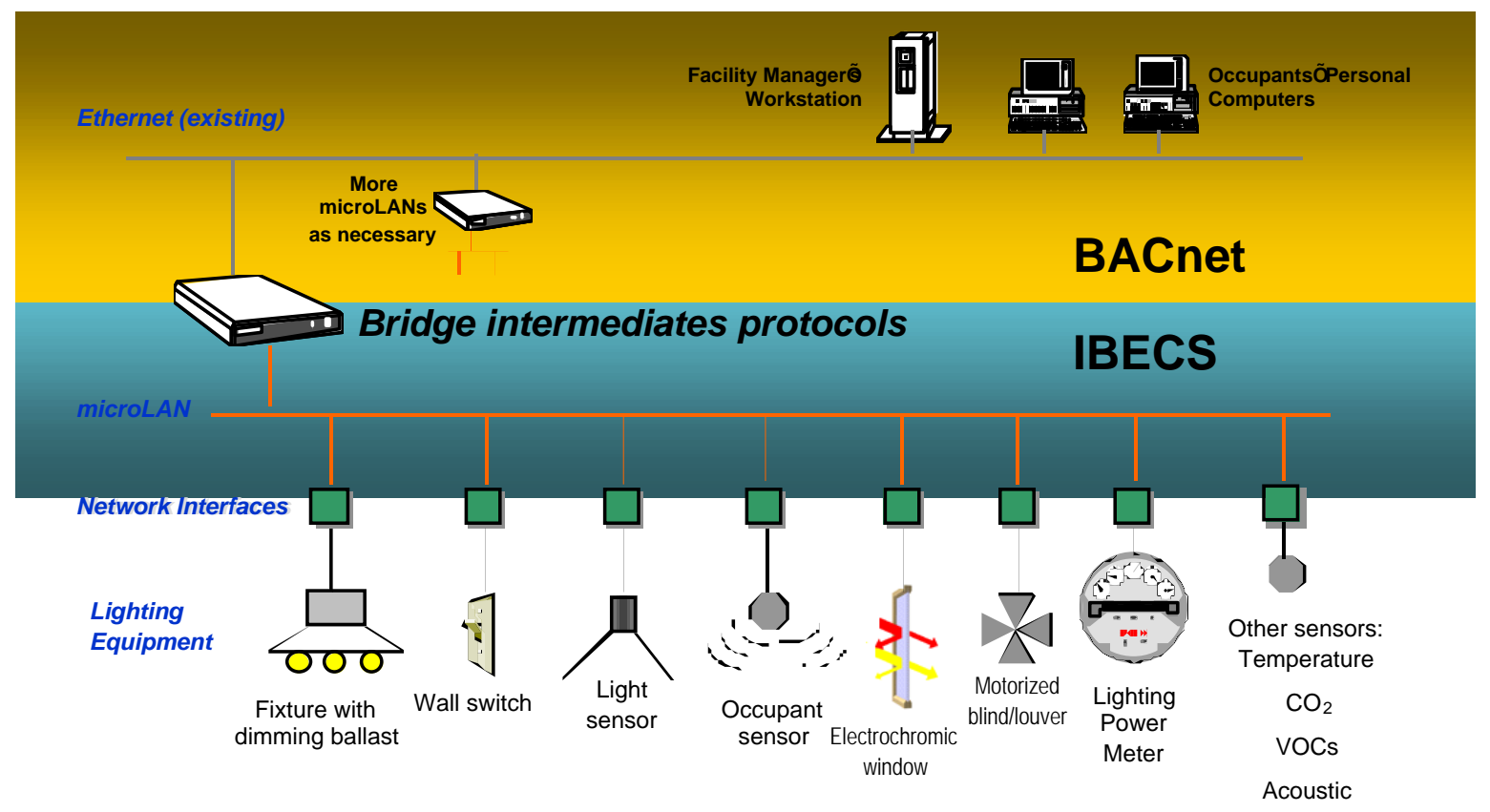

Figure 5.5. Relationship between BACnet and IBECS. BACnet deals with the upper-level communications protocol, while IBECS handles the lower part of the network. A bridge intermediates between the two protocols.

Even though BACnet need not be extended to control each small end node, the basic data structures and objects that BACnet defines can and should be implemented on the MS network. This simplifies the 
programmer's task, since the BACnet data structures and priorities are preserved across the bridge even if the entire protocol is not implemented at the end node level. For example, the Java data structures that IBECS uses will effectively mirror native BACnet objects.

\subsubsection{BCS Installation}

There is another issue that merits discussion and that is the system in place for design and installation of BCS networks. Presently this falls largely into the hands of BCS contractors or higher expertise consultants on larger projects. The difficulty is that the construction industry is famously slow in applying new technology. And there is a steep learning curve associated with all the new options-not only implementing devices that connect to the corporate LAN, but also understanding security issues and options related to traversing the internet, and the difficulties of interfacing with IT departments and outside service providers. If the BCS is no longer an independent system, then neither is the BCS contractor able to work in isolation. How do we educate contractors and building facility engineers? What is the best model for getting the right networks in the right places and commissioned and maintained properly? And how do we move toward that model in the real world of building construction and operation?

\subsubsection{Proposed Research}

1. Investigate different potential applications of distributed networks, including AFDD, sensor sharing among BCS sub-systems, automated commissioning, etc. Examine required network bandwidth, response times, data needs (sensor performance and sensor count), and scalability requirements. Are gateways required? What features are required in the gateway (translation, storage, security, control, etc.)? What tools are required to configure and manage the system/application? (near term)

2. Analyze performance of integrated building systems (lighting, HVAC, other) using models of the networks and systems.

3. Develop, test, and demonstrate a robust network cabling system for introducing low-cost building equipment control networks into commercial buildings. Need to standardize on the type of cabling, connectors, co-existence of network cabling and power wiring in same conduit. System should be fault tolerant in real time (near term).

4. Examine how existing IEEE Standards such as IEEE 1451 Standard on Sensors and Actuators serve as a basis for standardizing the storage of digital data on application-specific sensors and actuators for building equipment. The TEDS (Transducer Electronic Data Sheet) tables in IEEE 1451 can be used to make "plug-and-play" operation a reality in commercial buildings (near term).

5. Investigate how to ensure that open protocol objects and data structures propagate into lowlevel protocols for small end node control (near term).

6. Address the BCS installation model issue. What design/build/service model is best to achieve best-practice networks that perform as designed with minimum maintenance, sufficient BCS/IT staff cooperation, ongoing education, etc.? And how do we move that model toward reality? (near term)

7. Develop cost-effective strategies for improving the robustness of a building network, such as the use of low-power backup systems.

8. Develop integrated building networks that support building-wide energy management as well as personal comfort control strategies. 
9. Develop standards that enable seamless transfer of information between distinct building systems (i.e., allow the elevator, lighting, HVAC, and other building systems to communicate in order to reduce peak power consumption).

10. Develop web-based technologies for observing and supervising building energy management as well as for remote monitoring for maintenance and service.

\subsection{Building Control System Physical Security}

\subsubsection{Background Need}

The physical security of buildings is taking on increasing importance for a variety of reasons, including concerns related to limiting access to authorized individuals and preventing criminal or malicious activities. Traditional methods for controlling access, such as keys, ID cards, tokens, or passwords, all have serious shortcomings that enable their circumvention by design or by accident. Keys can be duplicated, ID cards and tokens stolen or falsified, and passwords guessed or otherwise obtained.

An increasingly attractive option for access control has been biometric systems. These types of systems base identity verification on a physical trait, such as fingerprint, hand geometry, or facial appearance. The obvious advantage of biometric systems is that the biometric features cannot be borrowed, copied or stolen (without extreme difficulty). When combined with an identity card and a password, they allow a high degree of confidence that a proper identification has been made.

An equally important aspect of access control is limiting access to building networks and computer resources to authorized users. This is becoming even more important as networks become more interconnected and the capabilities expand for obtaining information and controlling actions, including the building automation system, via the building networks. The flexibilities afforded by interoperable building subsystems also allow more opportunity for unauthorized or unintended manipulation of the building automation system. At the same time, service technicians will need the capability to access the building automation system from remote access points. Identifying and limiting access to authorized individuals, and determining the range of privileges they should be afforded, is a critical function in this regard.

A third important aspect of access control is related to emergency responders, such as police, fire, or medical personnel. Depending on the type and scale of emergency, it may be necessary to have special procedures for verifying the identity of such emergency personnel, especially first responders, in order to adequately verify their identity without compromising their mission.

In order to achieve the best utility from biometric systems for controlling building physical and virtual access, the biometric systems must have a number of characteristics and capabilities, as follows:

1. The biometric system must be integrated with the building automation system (BAS).

2. There must be interoperability between different biometric devices and between the biometric devices and the BAS.

3. There must be methods for transferring and storing the biometric data in order to allow multimodal, distributed biometric systems. 


\subsubsection{Current Status}

Current research activities in biometrics are primarily focused on improving the accuracy of individual biometric verification methods and developing algorithms for performing multimodal biometric verifications. This research is primarily being pursued at universities such as San Jose State, Michigan State, and Carnegie Mellon and is funded by DOD, Immigration and Naturalization Service (INS), and State Department sources.

Other work is addressing the need for lower cost equipment, and the incorporation of biometric data on "smart cards." This work is also mainly being conducted at universities. The Biometric Consortium, headed by National Institute of Standards and Technology (NIST), has been established to help coordinate and disseminate biometric research results and application information. A NIST research project is also looking at enhancements to BACnet to accommodate biometric systems, and methods to interface with emergency responders. This would allow for interoperability, significantly reducing barriers to the integration of biometric systems with the BAS.

\subsubsection{Proposed Research}

1. Develop methods for integrating biometric systems with the BAS.

2. Enable real-time multimodal biometric verifications that are link to an occupant log database.

3. Create an integrated intelligent security system, capable of controlling and monitoring building access and combining that information with other sensor readings to make higher level assessments of security and safety threats.

\subsection{Building Control System Network Security}

\subsubsection{Background Need}

In existing building control installations, security is generally not a major concern. This is true because the building control system (BCS) is not connected to the corporate network and is not connected to the internet. In addition, the assumption is that not many people know the existing communication protocols; therefore, they cannot "hack" the system. The system components are generally in locked rooms inaccessible to the public. The operator's interface may have password-protected access control. This level of physical security has been sufficient. The lack of network security has not been a concern because the network is isolated and physically secure.

However, this situation is changing as building networks open to outside connections. It is becoming more common to link separate buildings within a campus environment using the corporate backbone that is already in place. That is, the BCS is largely separate within an individual building, but at some point (or points) the BCS connects to the corporate LAN or WAN to make the connection to the neighboring building. This exposes the BCS to threats from company employees (insider threats) and then via the corporate connection to the internet, exposes the BCS to attacks from outsiders through the corporate firewall.

Connecting multiple buildings in this way has benefits: allowing the facilitiy manager to monitor and control the BCS of different buildings while sitting in one seat; using the existing corporate network avoids additional infrastructure cost; connecting to the internet allows off-site partners to have access to 
data from BCS devices. Nonetheless, security becomes an issue that must be addressed using different available IT solutions: firewalls, VPN (Virtual Private Network) connections to remote equipment, and closing ports, to name a few.

\subsubsection{Current Status}

Notably absent is any security framework within the different BCS communication protocols. Although there is some mention of security in BACnet and LON, (a networking protocol for buildings) it is weak and not generally implemented in commercial products. Beyond this there are no recommended best security practices for network configuration and connections to outside networks. There is no IT security hardware designed for the BCS (e.g., a BACnet firewall). And most building owners do not have security policies in place to protect the BCS.

There is substantial need for an understanding of how best to secure the BCS as the control model continues to evolve. Research is needed to strengthen protocols, to educate building owners, to implement secure devices and systems, and to address secure connections to outside facilities.

As indicated above, perhaps the greatest issues in network security are those of defining security within the control system protocols and understanding the greater security landscape of connecting outside the building.

First, there are network security issues that are not related directly to connecting to the corporate LAN and internet. Among these are the network aspects of physical security, e.g., the biometrics system network interface and the communication and authorization challenges of allowing an emergency responder to have administrator privileges on the BCS network while they are in transit.

Additional challenges come from the need for control network stability with the presence of faulty devices, over-loaded networks, equipment failure, power interruptions, poor device software design, faulty device and network configuration, etc. Work is proceeding on fault detection and diagnostics and network configuration for security and interoperability (Holmberg 2003).

In addition to this is the already mentioned work on the protocol itself-necessary for ensuring security in facilities with higher security needs as well as for installations with connections to the corporate LAN and to the internet.

The push to develop security within the BCS protocol is mainly due to the connection to the internet. Presently, BCS vendors are making cautious efforts to offer BCS web access from off-site. The most secure application is only allow operation data to be transferred out of the building (viewed but not controlled). If a building owner wants control from outside, then some security must be provided to protect the BCS from others who would want to control the building, or damage the system.

Concerted effort is needed among different groups to understand how to implement security, and to share experience in protocol and network and inter-network design for security. As models of different secure network configurations emerge, protocol, software, and hardware can be designed to the model. Common Criteria (CC) Protection Profiles (PP) can be written to specify desired hardware security features, and educational materials can be prepared to help users understand security issues and recommended practices for attaining different levels of security. 
What we see is that building control is moving toward greater connectivity and services that will increase control options, improve operation and maintenance efficiencies, and allow for data exchange between multiple independent businesses that have need for building information, enabling greater energy efficiency and productivity. What has been already said above and in Section 2 of this report bears repeating: we cannot optimally address the security of this interconnected building control and communication system until we have a better grasp of such a complex system and what that system needs to look like.

What is clear is that there will be many security challenges as we try to find the balance between open communication and guarded security. We know we need to address security in terms of layers: both "security in depth" (i.e., different overlapping technologies to guard the network perimeter), as well as security at different layers of the communication stack-in the transport layer and network layer, over the internet and in the building, at the firewall, and at the access control panel of a more secure device or subsystem, from domain to domain, and domain within domain. Security must be comprehensive, seamless, and simple. It must leverage the work of the greater IT community as much as possible. A large variety of participants must work together to have any hope of interoperability.

\subsubsection{Proposed Research}

1. Security must be addressed in each analysis of network configurations that includes information exchange with service providers other than utilities. What data needs to be passed with what restrictions, and how must the network must be constrained (designed) for stability and security?

2. Threats will continue to evolve and will largely be those of the internet, and those faced by businesses in general. BCS users and vendors will need to address these threats by developing guidelines for securing BCS devices. Work is required to lead this effort and prepare documents such as model security policies and BCS hardware protection profiles according to the Common Criteria.

3. Research to define an integrated intelligent security system. The methodologies and strategies must be developed for enabling real-time multimodal biometric verifications and linking the verification events to an occupant log database. Along with this is required work to ensure interoperability between biometric systems and integration into the BCS network.

4. Continued research is needed to address security within the BCS protocols: to provide authentication, authorization, and encryption of messages between devices on the network, and to define secure implementation strategies. How is security across the internet best achieved for different applications (e.g., BCS-to-BCS vs. BCS-to-Service Partner)? How is security best addressed inside the building? Where are the threats?

5. How can end-to-end security be assured? What methods of authentication and encryption are appropriate for BCS applications (e.g., SSH, IPSec, SSL) ${ }^{1}$ What levels of access control are required? What is the best system for change control and documentation (e.g., archives of which users have changed setpoints or schedules and the dates associated which the changes)?

\footnotetext{
${ }^{1}$ Secure Shell (SSH), Secure Socket Layer (SSL), Internet Protocol Security (IPSec)
} 


\subsection{Protocol and Standards}

\subsubsection{Background Need}

As the building control system evolves over time it is clear that the "where are we headed?" question has a strong influence on protocol development efforts. As industry develops new services, new transport (i.e., web services), new connections (to utilities and others), new data constructs to support these connections, and new control models, the BCS protocol must evolve also to support these new areas. What we want is growth along a planned path rather than haphazard uncoordinated efforts that will result in security holes, non-interoperability, and other unforeseen problems.

It is already a challenge to identify minimum requirements for interoperability. As the control network grows in complexity, so must the protocol—with new services and data objects—perhaps evolving in major ways to match major changes in the global control network. These changes ultimately affect interoperability efforts. For example, security must be addressed as the BCS is exposed to new connections, but security will affect interoperability. If Building 1 is a very secure facility and Building 2 operates at minimum security, how is information exchanged? Can we send data securely from Building 1 to a secure sub-net in Building 2 when the VPN to the building ends at the BCS firewall, but the main network of the BCS does not offer high enough security? Or, for another example, what happens when the protocol allows multiple addressing formats to allow easier communication with a utility, but only some devices support the newest formats? Interoperability must be addressed throughout the process of growing the protocol.

As controls become more advanced, the protocol must be extended to accommodate the new controls and control models. As control becomes distributed, the protocol must adapt to support all the new demands placed on it. Research is needed on the "where are we headed" question in order to plot an ordered growth of the BCS protocol. R\&D in protocol interoperability and security must also continue, and efforts must be made to tie in with what other groups are doing in these areas.

We must ask what other standards outside of the BCS protocol are required. Where are the interfaces and what do those look like? What are the boundaries of the system? Who owns what? What format will be used to exchange data, what data will be exchanged and how will it be exchanged? What rules govern the system?

\subsubsection{Current Status}

BACnet is an ASHRAE standardized protocol (ANSI/ASHRAE 2001) that allows data communication and control of a wide range of building equipment, including HVAC, fire and alarm systems, security, elevators, and lighting. The BACnet protocol provides a basic structure that allows relatively easy interface of these different energy management systems. To facilitate data communication between various types of equipment, BACnet specifies an object-based communication model that enables these devices to "look" and "act" the same on the network. BACnet also specifies a network layer protocol that determines how communication between different network types is accomplished.

BACnet was originally designed to be flexible and extendable. It continues to evolve as it is implemented by more vendors in new and diverse applications. In present buildings, the most common application of 
BACnet is for controlling the HVAC system, with a tie-in to the fire system to allow HVAC response to fire alarms and a hard-wired connection to the elevator to shut it down in the event of fire. In more advanced buildings, there will be networked communication between a building control center and the HVAC, fire, access control, lights, and elevator systems.

Native BACnet lighting, fire, and access control systems are presently available and growing in sophistication and market share. The future in building control will likely be the use of BACnet in all building control sub-systems at some level. This requires growth in the protocol to accommodate the needs of different controls segments (e.g., secure communications for access control systems or sophisticated alarming features for the fire system). There are demands on the control technology as well—presently lighting systems do not need to have the relatively heavyweight BACnet protocol running at the sensor and light ballast control level, but instead the lighting control panel acts as a gateway to interface with the BACnet control system. This will likely change over time as control technologies advance.

There are other areas in which the protocol is being stretched and in which it may be stretched. One area is that of object definition-defining common models in XML schema that will allow machines to query an object profile of a vendor-extended object in order to allow discovery of additional features. This supports interoperability and robustness. Another potential area of growth is in the area of plug and play devices and sensors. How do we add a device or sensor to the network and have it recognized, configured, and known by other devices? This will require a new effort in the standards committee.

As discussed in the previous section, security is an ongoing development issue as well as a future research issue. Within the protocol itself there is a need for basic and advanced authentication and authorization. Advanced security would also allow for encryption of traffic on the wire. The BACnet Network Security Working Group is considering a simple password-based security model as well as a more secure Kerberos-based authentication and encryption model. These will allow different levels of security as required by different building owners. 1

Significant challenges lie ahead in implementing these security services in BACnet. The effects on interoperability must be addressed. In addition, there will be new security issues that arise as the BCS is linked with outside partners, and these need to be recognized and planned for to avoid holes and enduring security weaknesses. There must be continued efforts to learn from others, implement available IT solutions when possible, and guide security enhancements in the standard according to future needs. And it is clear that the control community cannot guide the development of the protocol in an optimal way without a clear understanding of where we are headed.

We do know that we are headed toward increased interaction with outside service providers that will involve two-way communication via some standard transport mechanisms and common data models. While BACnet offers communication over IP via the BACnet/IP service, this is designed for one BACnet network device to talk to a device on another network with a segment of the internet in between. It is very likely that BACnet will not be used for communication between the BCS and service providers such as a utility. Instead, there will need to be agreement on message formats to be exchanged and the order of

\footnotetext{
${ }^{1}$ Holmberg, D.G., 2003, “BACnet WAN Security Threat Assessment,” BACnet committee SSPC 135 document number DGH-001-2, under review for publication as NIST internal report.
} 
those messages and other rules of communication, and then transporting of those messages will use standards like web services.

Currently the BACnet Utility Interaction Working Group is working on enabling several new services that were identified in the 1999 ASHRAE Research Project 1011-RP, "Utility/Energy Management and Controls System (EMCS) Communication Protocol Requirements” (Kintner-Meyer and Burns 1999). That report discusses such needed interaction scenarios as load control, energy efficiency monitoring, quality of service, revenue metering and billing, air quality monitoring, dynamic demand bidding into the power exchange, and others. The working group is steadily addressing these scenarios. Current efforts are focused on load control, data presentation (allowing BACnet to make complex data structures network visible), meter interaction, and real time pricing (Holmberg 2003; Gemmill 2003). Efforts are needed to continue to look down the road and see where we are headed and how the standard needs to evolve to meet future challenges.

\subsubsection{Proposed Research}

1. For the short-term (FY04-FY05), demonstrate small-scale demonstration of the selected energyinformation services (such as remote energy efficiency monitoring, remote diagnostics, and curtailment and demand responsive services) using communication means proposed by ASHRAE research.

2. Analyze scalability and latency issues for communications between buildings and service provider on a large scale $(1,000,000)$ using diverse controls platforms ranging from BCS in large facilities to smart thermostats in small commercial buildings. (FY04-05)

3. Work with other stakeholders such as ASHRAE, EPRI, and IEEE to develop communication standards for energy services. (FY05)

4. Develop standard protocols and infrastructures that will enable remote monitoring of building systems for use in fault detection and diagnosis as well as forecasting of maintenance and service via prognostics.

5. Develop robust protocols that allow interaction with the grid without compromising building system operation.

6. For the mid-term, publish competitive solicitation for the communications and controls solution for all GSA sites using emerging communication standards. (FY06-08)

7. Extend security framework in BACnet (FY04-FY05) to include different levels of authentication, as well as a mechanism for authorization and encryption. Design authorization such that different sub-systems (e.g., access control and HVAC) can share data leading to integrated smart security.

8. Extend open protocol functionality outside building to service providers by providing necessary interface and data formats, etc. as required for different service providers (utilities and others). This may be via web services or by some other transport and may include definition of XML schema for individual services. (This is near to mid-term research as it must follow external standards developments.)

9. Develop low-cost-per-node open protocols. 


\subsection{Automated Diagnostics, Performance Monitoring, COMMISSIONING, OPTIMAL CONTROL, AND TOOLS}

\subsection{Overview}

Performance monitoring, automated fault detection and diagnosis, commissioning, optimal control and the use of development environments, design tools and trainers are complementary, and in some respects synergistic, technologies that have strong potential to realize significant energy savings and other performance improvements in commercial buildings, including existing buildings. There is a significant body of previous R\&D relating to these technologies that indicates their potential, both generically and for specific approaches and methods. In a significant number of cases, there is the opportunity to establish R\&D programs and projects that leverage this existing work in order to move relatively quickly to tools that can be deployed in the marketplace. In other cases, significant additional research is needed before viable prototypes can be produced and costs and benefits assessed. Taken together, there is a very significant opportunity for DOE to establish a program that will have a substantial positive impact on the way in which buildings are operated.

It is increasingly widely recognized that buildings in general, and commercial buildings in particular, fail to operate as intended by their designers or at the performance level of which the fabric and the equipment installed in the building are capable. In addition to a considerable body of anecdotal evidence, the main objective evidence of this situation is the reduction in energy consumption that typically results from retro-commissioning activities such as those pioneered by Texas A\&M University (Gregerson 1997).

Lack of properly trained operators and high turnover of building operators also contributes significantly to inefficient operation and maintenance. Although lack of feedback about performance of building systems contributes to inefficient operations, providing feedback without providing them incentives to correct improper operation does not work either (Pratt et al. 2003 and Katipamula and Gaines. 2003). Therefore, educating operators, building owners, managers and engineers on the benefits of using the advance technologies is also required.

In addition, it is known that many new energy-saving technologies and approaches from research fail to be adopted in the marketplace. Thus, there is a need to understand the building operations industry, in particular the building life cycle cost structure and the decision-making process regarding the adoption of advanced technologies. This understanding could be used to put together a value proposition that makes clear the potential benefits of using these technologies for all parties involved, and clear the way for selfconfiguring, adaptive controls that autonomously maintain close to optimal building performance and identify operational faults on a continuous basis.

\subsection{Organization and Structure}

A variety of complementary approaches to preventing or remedying this situation have been proposed and are either under development or in the early stages of deployment; the most appropriate choice depends on the type of building and the way in which its operation is managed. This chapter is structured to reflect one way in which these approaches can be categorized: 
- Performance monitoring involves continuously measuring the behavior of the building in order to assess its performance. Issues include the provision of the necessary sensors and data acquisition, storage, retrieval and visualization. It also includes the role of human intelligence in assessing performance.

- Automated fault detection and diagnosis involves the use of software tools to analyze the behavior of the building, determine if the performance is unsatisfactory (fault detection), and then isolate or localize the fault in order to facilitate repair (fault diagnosis). The behavior may be observed in the course of an active functional test performed by the tool or in the course of passive monitoring of routine operation.

- Commissioning typically involves active testing of components and sub-systems as one of its core activities, although it also includes a systematic series of activities, starting in the planning phase, aimed at ensuring correct operation of the building. Commissioning is currently a manual activity involving specific tests that are performed and analyzed by specially trained engineers. Automated commissioning, which is a relatively new research topic, is based on active functional tests performed and analyzed by an automated fault detection and diagnosis tool.

- Optimized control-performance may be improved by optimizing the control strategy, either through the use of on-line optimal control or by the use of heuristic strategies that approximate optimal control.

Efficient development of these methods, and tools based on these methods, can be facilitated by simulation-based 'virtual building environments' that allow repeatable, flexible, and inexpensive testing in the laboratory before proceeding to field trials. This chapter discusses the needs for, and expected benefits from, these technologies, summarizes current R\&D in each of these areas, and presents recommendations for future $\mathrm{R} \& \mathrm{D}$ activities.

There is significant interaction, and some overlap, between the technology areas listed above. The sensors and the data acquisition capabilities required to monitor the energy performance of buildings for analysis by operators and engineers are also required by automated fault detection and diagnosis (AFDD) methods in order for them to detect some important faults that impact energy performance. The methods used in AFDD to analyze the behavior of buildings and HVAC systems are applicable both to the active testing used in commissioning and to the passive monitoring used in routine operation. The models used in model-based AFDD can also be used in model-based control optimization procedures. These linkages should be recognized and exploited both in an R\&D program and in producing integrated solutions for deployment in buildings.

\subsection{Performance Monitoring}

\subsubsection{Background Need}

Recent studies (ELCAP, Texas LoanSTAR, and IMDS) have shown that metering and monitoring energy end-uses and analyzing them on a continuous basis can have an enormous benefit in terms of detecting and correcting operation problems (Taylor and Pratt 1989; Piette et al. 1998, 2000, 2001, 2003; Taylor 1992; Claridge 1998). However, continuous monitoring can be costly and time consuming unless the data collection is automated. 
Performance monitoring is defined as a process of continuous measurement to support building energy analysis and control. These continuous measurement systems include sensors, polling frequencies, data archival and retrieval systems, data visualization, and statistical analysis tools that provide an interface to the data. While typically based on or related to the sensors in control systems, performance monitoring tools may include electric load measurements, dynamic energy costs, and multiple buildings. The primary difference between the performance monitoring systems and AFDD systems is that they are intended for manual or visual analysis. By contrast, AFDD systems generally provide notification of a fault in an HVAC system. Traditionally, BASs have provided loop control, alarm reporting, dynamic control, and energy management functions. BASs could provide valuable additional services to building managers and operators by collecting, storing, and continuously and systematically analyzing and drawing conclusions from key measurements.

BASs have limited capabilities to collect, archive, and organize HVAC and related data. There is a great need to evaluate the abilities of the underlying information systems infrastructure to support performance monitoring, automated diagnostics and commissioning, and advanced controls. Data polling frequencies, data quality issues, archival and database management systems, file structures, data access issues, and web-based remote access are all important issues that need to be evaluated as we attempt to better utilize data from BASs and related systems for performance analysis of commercial buildings.

There is also a need to standardize the performance metrics used to assess building performance and integrate this activity with the BAS data analysis and visualization. Issues with current practices include inconsistent measurement and archiving methods, inconsistent boundaries for energy measurements, and inconsistent normalization techniques.

Although there has been some development activity by third-party developers and researchers, the lack of easy access to data from sensors and meters through BASs has impeded widespread acceptance of such tools and analysis methods. In recent years, many manufacturers have started supporting standard protocols such as object linking and embedding (OLE) and its predecessor dynamic data exchange (DDE) for application-to-application data exchange and communication using data objects. In principle these protocols should provide simpler means for data collection from BASs; however, this has not been the case in the field because ostensibly minor differences in implementation can cause significant problems. Also standard protocols, such as BACnet ${ }^{\mathrm{TM}}$, ASHRAE/ANSI Standard 135-2001 [developed by ASHRAE and adopted by ANSI] (ANSI/ASHRAE 2001), can contribute to easier implementation by providing standard mechanisms by which applications can communicate with field devices and different BASs can communicate with each other.

The new standards and protocols can potentially help new installations, but will not solve problems with existing building installations. Alternative technologies that bridge legacy systems in existing buildings to new technologies, such as gateways, are required.

Operational problems associated with degraded equipment, failed sensors, improper installation, poor maintenance, and improperly implemented controls plague most commercial buildings. These conditions can be detected by operators using visualization tools that constantly monitor the performance of the system or sub-systems. Automated tools can help in detecting and diagnosing faults; however, a slow 
degradation in performance may be difficult to detect. In addition to monitoring the system performance, there is also a need for tools that monitor and analyze building peak consumption and indoor air quality.

\subsubsection{Current Status}

Over the last two decades, there have been many uncoordinated research and development activities in the general area of performance monitoring in commercial buildings. Research and development activities related to development of analysis methods have been limited to national laboratories (Taylor and Pratt 1989; Taylor 1992) and universities (Claridge et al 1996); although in the past few years private industry has developed some limited applications. The research and development in this general area can be broadly classified into the following categories:

- monitoring technology development

- technology evaluation

- applications development

- development of analysis methods

- data access tools

- technology innovation and adoption analysis

- standardization of performance metrics.

\subsubsection{Monitoring Technology Development}

Over the past few years, several applications that monitor end-use consumption with limited diagnostic or analysis features have been developed by the private industry. These applications can be classified into three groups (Motegi and Piette 2002):

1. Demand Response Systems (DR Systems) - DR systems are oriented toward managing electric load curtailment events and act as an interface between building and electricity suppliers. These systems are often limited in the breadth of applications beyond DR.

2. Energy Information Systems (EIS) - EIS, like DR systems, have evolved with metering systems provided by electric utilities, but they offer new and important functionality for tracking and managing energy. They are primarily targeted for energy budgeting activities and lack advanced analysis or diagnostic capabilities.

3. Web-based Energy Management and Control Systems (Web EMCS) or Building Automation Systems - Web-EMCS are the emerging class of EMCS that offer web connectivity for monitoring and control. Most of the monitoring capabilities are limited to providing access to the trends logs.

In addition to application development, several control manufacturers and third-party developers have been active in development of gateways to improve interoperability between different controls systems and applications (http://www.automatedlogic.com/, http://www.enflex.com/, http://www.tridium.com/, http://www.pwrm.com/ and http://www.envenergy.com/).

The U.S. General Services Administration (GSA) is developing the GSA Energy and Maintenance Network (GEMnet), which involves integrating performance monitoring with numerous other applications (Piette et al. 2000). The objectives of the GEMnet system are to interface real-time applications such as BAS and lighting controls with a centralized maintenance management system; to 
data warehouse information derived from many systems and buildings; to provide a framework for performance benchmarking, data analysis and building diagnostics; and to provide mechanisms for centralized support of automation systems.

Researchers at Pacific Northwest National Laboratory (PNNL) have shown that real-time monitoring of a central plant can save as much as 25\% in energy use (Meador and Jarrell 1999). Decision Support for Operations and Maintenance (DSOM) is a software tool developed by PNNL that allows online condition-monitoring of equipment and provides early warning signs of degraded performance (http://www.pnl.gov/dsom/DSOMfinal.pdf). It is designed to allow operators to make informed decisions about how to operate their plant more efficiently.

Enron Energy Services (EES) realized that performance monitoring is a key element in operating facilities optimally and conducted a comprehensive search of applications and infrastructure to support the activity. Although EES found many energy information systems, none of them provided flexibility and scalability required to monitor several buildings from a central location. Therefore, EES started to develop its own energy information infrastructure and was successful in demonstrating the usefulness of performance monitoring.

\subsubsection{Technology Evaluation}

Technology evaluation research is focused on understanding how to best use new performance monitoring technology with emerging information technology. As internet technology, database management systems, and gateway technology evolve, they provide new enabling features for both manual data analysis and more sophisticated automated diagnostics.

Lawrence Berkeley National Laboratory (LBNL), with funds from The California Institute for Energy and Environment (CIEE), DOE, Sacramento Municipal Utility District (SMUD), and the California Energy Commission (CEC), has been evaluating the value of high quality performance monitoring and data visualization tools in office buildings (Piette et al. 1998, 2000, 2001, 2003, http://imds.lbl.gov). The Information Monitoring and Diagnostic System consists of a set of high quality sensors, high frequency polling, a robust archive and data retrieval system, along with powerful data visualization tools on site and over the web. The IMDS has been shown to be of great interest for daily use and review of control strategies by building operators at two different sites. The data have also been shown to be valuable in overall building energy analysis and retrofit planning. LBNL has also evaluated several performancemonitoring systems (http://buildings.lbl.gov/hpcbs/pubs/E5_49977.pdf).

Ultimately these tools become the foundation of "continuous commissioning” methods to continually track energy performance and other building parameters to ensure efficient operations. The Northwest Energy Efficiency Alliance has also been conducting research on such a tool. One such example is their evaluation of PowerNet (http://www.powernetsofware.com and http://www.nwalliance.org/resources/projectwebsites.asp).

\subsubsection{Applications Development}

There are numerous projects that work with the time-series building performance data to organize it into useful methods for analysis. DOE's Whole-Buildings Road Map outlines the need for Performance Metrics, which requires Performance Monitoring for long-term data development. LBNL, funded by DOE and CEC, has developed Metracker, to help define methods to organize and archive key building 
performance metrics. This tool is oriented toward performance metrics tracking over the building life cycle, from design to commissioning and operations. Other efforts emphasize performance monitoring during ongoing operations (http://eetd.lbl.gov/btp/buildings/hpcbs/Element_2/02_E2.html).

\subsubsection{Development of Analysis Methods}

Development of analysis methods for performance monitoring and estimating savings from retrofits has been an active area of research at universities (Claridge et al 1996) and national laboratories (Taylor and Pratt 1989; Taylor 1992). The analysis methods for estimating savings from energy-efficient retrofits using measured performance end-use data have evolved over the past two decades. The International Performance Measurement and Verification Protocol was a result of much of this effort (IPMVP 2002).

Deregulation of electric utility industry represents opportunities and challenges to the building operators and owners. In deregulated areas the public utility commissions, federal energy regulatory commission, and independent system operators have been promoting demand response programs (DR) to make electricity supply more reliable. These DR programs require development of a baseline and proof of load curtailment for payments. The analysis methods developed for estimating retrofit savings are not always appropriate; therefore, new methods have to be developed or existing methods enhanced for estimating the load curtailment savings.

\subsubsection{Data Access Tools}

Without easy access of data from sensors and meters, the automated tools are of little value, and installation of custom data acquisition systems is not cost effective. While the existing building automation systems can be leveraged to get access to the sensor and meter values, non-standard protocols and proprietary protocols make it difficult to design and build universal data access tools. Many thirdparty entities have tried to provide such functionality, but with limited success.

A data collection tool was developed as part of the Whole Building Diagnostician software package, which was developed as part of the commercial buildings research program of the U.S. Department of Energy's Building Technologies Program. It was further enhanced as part of the CEC Public Interest Energy Research (PIER) program (Brambley et al. 1998 and Katipamula et al. 1999). The data collection module was designed to work with any BAS that supports DDE (dynamic data exchange) or BACnet protocols. However, in the field tests it successfully worked with some BASs and had difficulty with others. Although the DDE and the BACnet protocols are standard protocols, there may be minor differences in the way they are implemented.

\subsubsection{Technology Innovation and Adoption Analysis}

There is a need to provide a description of the innovation adoption process and a road map for the adoption of new controls technology along with performance monitoring technologies for practical business applications in the buildings industry. To date, limited research has been conducted in this area (Piette et al. 1998, 2001, and Shockman and Piette 2000).

\subsubsection{Standardization of Performance Metrics}

There have been numerous efforts in the area of performance metrics, but they have not been coordinated. Performance metrics are essential for benchmarking performance but they depend on the availability of performance data. The National Renewable Energy Laboratory (NREL), with funding from DOE, has 
initiated an effort to survey existing methods and standardize the measurement and characterization of building energy performance. This project is working closely with other efforts in this area including LBNL, Oak Ridge National Laboratory (ORNL), ASHRAE, ASTM/ISO (American Society for Testing and Materials/International Standards Organization), and the international Green Building Challenge in an attempt to reduce the disparity in this area.

\subsubsection{Proposed Research}

The goal of the proposed research program is to research, develop, demonstrate, and encourage deployment of enabling technologies that enhance building operation and controls through performance monitoring. DOE should continue, or consider initiating new R\&D activities, in the following areas:

1. Encourage control manufacturers, industry groups, and other third-party developers to form a consortium to address data access issues, by1) developing a framework for development of universal data acquisition tools, 2) encouraging manufacturers to adopt and support a common data exchange protocol, 3) developing test modules for commonly used protocols (DDE, DCOM, OLE, XML), and 4) field testing the modules. This idea already has some support in the industry - see http://www.automatedbuildings.com/news/jan02/art/alc/alc.htm. This can be completed in the near- to mid-term.

2. Support development of data analysis methods and tools. Proposed energy policy legislation would require electric loads greater than $50 \mathrm{~kW}$ to have interval meters. For example, develop analysis tools that continuously monitor end-use consumption, compare it to expected consumption, and create exception and trend reports. These methods should be available to use directly without having to buy expensive application suites. These analysis techniques should include peak demand and load shape monitoring to support strategies to minimize demand when electric costs are high. This R\&D activity can be completed in the near-term.

3. Instigate research that integrates energy monitoring with non-energy performance data such as maintenance activities and equipment life, comfort and tenant complaints, and indoor air quality. Linking the development of energy performance monitoring to non-energy measurements will help bring additional value through integrated building systems performance monitoring. This work can be completed in the mid term.

4. Support development of tools that provide continuous performance feedback. Also support work with building owners and mangers to devise ways to provide incentives and encouragement to building operators to correct the problems reported by the tools. This R\&D activity can be completed in the mid-term to produce methods and prototype applications for developers to incorporate in their applications suites.

5. Pursue research to explore and better understand the technology adoption and decision making environments to accelerate the use of enhanced performance monitoring and control systems. In particular, support the detailed development of a value proposition that includes a building life cycle cost analysis highlighting the potential benefits from the use of advanced technologies. This work can be completed in the mid term.

\subsection{Automated Fault Detection and Diagnosis}

\subsubsection{Background Need}

Many buildings fail to perform as well as expected because of problems associated with degraded equipment, failed sensors, improper installation, poor maintenance, and improperly implemented controls. Today, most problems with building systems are detected as a result of occupant complaints or alarms 
provided by building automation systems (BAS). Building operators often respond inappropriately due to their lack of understanding of complex control strategies. Building systems maintenance, as usually performed, is notably ineffective, being almost exclusively complaint-driven and "quick fix" oriented. This is especially true for problems affecting air quality and efficiency because they are "silent killers" that go unnoticed until complete system failure occurs.

Automated fault detection and diagnosis (AFDD) is an automatic process by which faulty operation, degraded performance, and broken components in a physical system are detected and understood. AFDD tools are based on algorithms that process data to determine whether the source of the data is experiencing a fault. The tool may be passive, analyzing operation of the equipment/system without altering any of its set-points or control outputs, or active, automatically initiating changes to produce or simulate operating conditions that cover a more complete range of conditions that might not be covered for some time under normal operation.

AFDD can be integrated into an automated commissioning process. Commissioning (new buildings) and retro-commissioning (existing buildings) involve functional tests conducted to determine whether a piece of equipment or system is operating properly. Generally, these tests are only performed during the discrete activity of commissioning — at the start-up of a new building or during retro-commissioning of an existing building. To pass the commissioning process, proper operation of the equipment is verified by observation of a series of functional tests. This process, however, does not ensure that the equipment continues to function properly. Only continuous monitoring of the status of the equipment and its performance can ensure continued proper operation.

The AFDD system is central to this continuous commissioning process by constantly monitoring the equipment and identifying a failure or degradation in performance. The human operator or repair person is still critical to completing the commissioning cycle, but, without the automated system monitoring continuously, problems can go undetected for days, weeks, months, or even years.

Automated commissioning and diagnostic technologies for building systems and equipment promise to help remedy these problems and improve building operation by automatically and continuously detecting performance problems and maintenance requirements and bringing them to the attention of building operators. By embedding the expertise required to detect and diagnose operational problems in software tools that leverage existing sensors and control systems, detection and diagnosis can be conducted automatically and comprehensively. These tools are not intended to replace the people operating the building systems, but rather to help them improve the operation of those systems.

\subsubsection{Current Status}

Currently, most building owners are not aware of the power of automated commissioning and diagnostic technology to provide them more cost effective, comfortable, and productive buildings. The technology is in its infancy and not yet well-known in practice. Because of the high first cost associated with the development of such systems, the industry has not embraced the technology. Finally, energy service companies that may eventually offer commissioning and diagnostic services are slow to expand their business practices beyond their current focus on lighting and equipment retrofits. Despite this current state, automated diagnostic and commissioning technology offers promise of a future with improved 
facility operation, better indoor environments, and enhanced and higher quality offerings by service companies.

AFDD was first established in the process, nuclear, aircraft, and automotive industries (Braun 1999). Because these critical processes require high reliability and operational safety, the FDD system is an essential element of plant operations. Early fault detection methods were generally limited to detecting values of measurable output when the signals had already exceeded the limit. Use of microcomputers in the early 1980s led to advanced mathematical process models, which provided the ability to detect the fault earlier and to locate the fault by the use of additional measurable signals (Isermann 1984). Because reliability and safety are primary concerns, these plants have extensive and redundant sensors. Therefore, the FDD methods evolved around the data-rich environment. At about the same time (late 1970s), fault detection and diagnosis was beginning to be applied to mass-produced consumer equipment such as automobiles and household appliances (Willsky 1976).

Much of the research in the buildings area has been conducted either at universities or at the national laboratories. The greatest number of FDD applications were related to vapor compression equipment (refrigerators, air-conditioners, heat pumps, and chillers) followed by application to air-handling units. The FDD methods used measured pressure and/or temperatures at various locations and then used thermodynamic relationships to detect and diagnose common faults.

In the early 1990s, the International Energy Agency (IEA) commissioned the Annex 25 collaborative research project on real-time simulation of HVAC\&R (Heating, Ventilation, Air-conditioning and Refrigeration) systems for building optimization, fault detection, and diagnostics (Hyvärinen and Kärki 1996). The Annex 25 study identified common faults for various types of HVAC\&R systems, and a wide variety of detection and diagnosis methods were investigated including physical and black-box models of HVAC\&R systems. Annex 25 was followed by Annex 34, the aim of which was to test and demonstrate AFDD in real buildings. The US was represented by NIST, Purdue University, Honeywell, Johnson Controls, and Field Diagnostic Services. Most of the FDD schemes included some form of expert knowledge and needed a significant amount of on-line manual tuning to make them work properly (Dexter and Pakanen 2001).

Annex 34 has been followed by Annex 40, which is concerned with commissioning HVAC systems for improved energy efficiency. The US is represented by NIST, LBNL, Texas A\&M University, Johnson Controls, and Siemens. One of the aims of the Annex is to develop and demonstrate automated commissioning methods and tools based on automated FDD. One specific focus is on model-based methods at both the component and whole building level. The Annex is due to finish at the end of 2004. Further details may be found at http://www.commissioning-hvac.org/.

There has been some work toward the development of evaluation methods for FDD. ASHRAE 1020-RP (Norford et al. 2002) involved the assessment and comparison of two different FDD methods at the Iowa Energy Center using blind testing with artificial faults in air handling units in an experimental building.

In April 2000, the California Energy Commission (CEC) initiated several projects to develop, evaluate, and demonstrate FDD techniques and tools. These projects are described briefly in this section. Results from these projects can be found at the following website http://www.archenergy.com/cec-eeb/. 
Purdue University has extended its previous work on roof-top packaged air-conditioners and heat pumps (Rossi and Braun 1996; Breuker and Braun 1999a,b). The extension includes development of an adaptive FDD modeling approach and consideration of issues such as determining when a unit is operating at steady state, understanding the influence of unmeasured variables such as solar radiation and rainfall, and determining under what conditions model "normal" parameters should be reset (e.g., after what level of maintenance).

Researchers from MIT have been investigating methods for using non-intrusive load measurement (NILM) methods to detect cycling and scheduling faults. NILM detection of cycling and scheduling faults is dependent on identifying unique signatures for the individual electrical loads. Initial field testing has been completed at the Iowa Energy Research Center and field testing is underway at six sites in California.

NIST has developed an AHU [air handling unit] diagnostic tool using AHU Performance Assessment Rules (APAR), which is a set of expert rules derived from mass and energy balances to detect common faults in AHUs (House et al. 2001; Castro et al. 2003). The APAR tool was found to be successful at finding a wide variety of faults including stuck or leaking dampers and control valves, sensor drift, and improper control sequencing. APAR has also been embedded in AHU controllers from several manufacturers. A multiple-site field evaluation of APAR, embedded in AHU controllers, is scheduled to begin in October 2003.

NIST has also developed a diagnostic method, based on statistical quality control measures, for detecting faults in VAV (variable air volume) boxes using Performance Assessment Control Charts (VPACC). The VPACC tool was found to be successful at finding a wide variety of faults including stuck or leaking dampers and control valves, sensor drift, and improper control sequencing (Castro et al. 2003). VPACC has also been embedded in VAV box controllers from several manufacturers

PNNL has conducted several field tests of a DOE-developed technology, the Whole-Building Diagnostician (WBD), for automatically and continually diagnosing operational problems in AHUs. In a recent demonstration at the Symphony Towers building in San Diego, the OAE (Outdoor-Air/Economizer module) diagnostician identified problems with all four AHUs, which were confirmed by visual/manual inspection (Katipamula et al. 2003b). These findings are consistent with the other field demonstrations of the WBD where the OAE found similar problems that should have been detected during commissioning. The building operators corrected only one of the several problems identified by the OAE diagnostician over a three-year demonstration period. The demonstration showed that diagnostic technology can provide useful information on equipment status but its value is not realized unless building staff implement fixes to the problems.

It is insufficient merely to identify problems and their impacts; building staff must correct them. If users are not proficient in using their control systems to correct problems, are too busy with other duties, or lack resources to obtain help from contractors, diagnostic technologies alone will not provide systemefficiency improvements. Furthermore, if operators do not clearly understand the value of correcting a problem, they are unlikely to fix it. In this respect, simulations are a valuable tool that can very effectively support operator decision making. Building models can be incorporated into virtual building environments and clearly show to the operator the potential benefits of addressing a problem. PNNL and 
PECI developed methods to automate commissioning process for AHUs as part of research sponsored by the American Refrigeration Technology Institute (Brambley et al. 2002 and Katipamula et al. 2003b).

LBNL has developed software for component-level model-based fault detection and is currently testing it using measured data from a number of commercial office buildings. The software includes a library of models for AHUs and chillers, which is being extended in IEA Annex 40 (see above), and a toolbox of routines required to turn a model into a model-based fault detection tool. The approach is to link design, commissioning, and operation by using design data to configure the models for use in commissioning and to use commissioning measurements to fine tune the models for use during routine operation.

LBNL, Texas A\&M University, and the University of Nebraska are developing techniques for using whole building simulation for fault detection and diagnosis. For new buildings, a program like EnergyPlus can be configured to represent the design and then used to predict the performance expected by the designer, both in commissioning and in routine operation. For existing buildings where the details of the original design are either lost or are no longer relevant because of changes in the use of the building, a calibrated simulation approach is used. A detailed simulation model, such as EnergyPlus or DOE-2, or a simplified model, such as AIRMODEL, is calibrated using operating data and then used to identify operational problems, such as simultaneous heating and cooling, and to refine the control strategy, e.g., change the reset schedules.

While AFDD was well established in the process, nuclear, aircraft, and automotive industries, it did not enter the building and HVAC\&R industries until the mid 1990s (Braun 1999). High reliability and safety are relatively less critical in building operations; therefore, AFDD did not generate the same level of interest among the building researchers, owners, and operators. Although AFDD has been an active area of research among the buildings and the HVAC\&R community for a number of years, it is not widely deployed in the field. The primary reasons for the lack of AFDD tools in the marketplace are the lack of interest in energy efficiency generally, the concomitant lack of demand from building owners, (also due to a lack of confidence about actual benefits), and uncertainties on the part of controls vendors and other possible who might commercialize the technology as to the robustness of current AFDD methods and the cost of configuring these methods for use in specific buildings. The primary drivers of building operations are still operating costs and capital investment, and AFDD has not yet been developed to the point where the benefits can be established or the costs of mature implementation assessed.

Although we have seen significant development from a theoretical point of view, the practical aspects of implementing AFDD systems in the field have not yet been thoroughly analyzed (sensitivity of diagnosis versus false alarm, data gathering). As noted earlier, lack of sensors and easy access to sensor data make field implementation of AFDD tools difficult and less reliable. Development of low-cost wired/wireless sensors, so that buildings and HVAC\&R systems become sensor-rich, will require significant changes in AFDD methods, and should make them more reliable.

\subsubsection{Proposed Research}

The principal technical challenges in AFDD are to develop diagnostic methods and prototype tools that:

- can be automated

- comprehensively diagnose the range and diversity of building systems and equipment 
- can be configured with the minimum possible effort and cost

- make use of a minimal set of additional sensors beyond those used for control

- $\quad$ are applicable for building commissioning, as well as on-going diagnostics.

The goal of the proposed program is to research, develop, demonstrate, and encourage deployment of the enabling technologies for prototype automated commissioning and fault detection and diagnostic tools to enhance building operation and controls. It is critical for DOE to coordinate this research activity with manufacturers and industry groups. In addition, DOE, with the help of stakeholders, should develop a market transformation process so that products developed from this research are adopted by industry and contribute toward enhancing building operation.

There has been considerable work done in this area, which can be leveraged and advanced to produce quick near-term results. In addition, there are several areas that have not yet been targeted by researchers. Therefore, DOE should continue or undertake new research and development activities in the following areas:

1. Develop and field test AFDD methods and prototype tools for AHUs, chillers, and VAV boxes. Activities would include comparison of methods and ranking them on their effectiveness and ease of implementation in the field. This R\&D activity can be completed in the near term.

2. Develop processes and procedures for automated continuous commissioning (automated functional testing) of HVAC systems and sub-systems. This activity involves not only development of AFDD methods and tools, but also continuous monitoring, evaluation of severity of the faults, and where possible making automated compensation of controls to correct improper operation. This R\&D activity can be completed in the near term to mid term.

3. Determine tradeoffs between the sensitivity of detection and the sensitivity to false alarms through limited lab and extensive field testing (exploration of what the detection/diagnosis thresholds should be). This R\&D activity can be completed in the near term to mid term.

4. Develop new AFDD methods for other HVAC systems (boilers, absorption chillers, microturbines, fuel cells). This R\&D activity can be completed in the mid term.

5. Document benefits from the use of AFDD and automated commissioning technologies. This R\&D activity can be completed in the mid term.

6. Develop a roadmap for implementing the AFDD and commissioning technologies that have been developed, aimed at securing widespread adoption. This R\&D activity can be completed in the mid term.

7. When several AFDD and automated commissioning tools are deployed in a building, there is a potential for lack of interoperability and conflicts can occur. Therefore, a mechanism to resolve conflict has to be developed. This R\&D activity can be completed in the long term.

8. Develop whole building models and tools for evaluating the performance and robustness of AFDD strategies.

9. Develop standard, secure protocols or infrastructures that will enable remote monitoring of building systems for use in fault detection and diagnosis as well as forecasting of maintenance and service via prognostics. 
10. Support the development of standardized building models and simulators, linked to AFDD software that can effectively support operator decision-making for improved building performance.

11. Develop, implement, and field test AFDD methods that can be automatically tuned and require minimal manual configuration. This can be linked to commissioning procedures that automatically produce a description of the desired behavior of the building systems.

12. Accelerate adoption of AFDD and continuous commissioning technology by supporting field trials that clearly demonstrate the technology and distribute the burden of development costs across the industry.

In addition to developing AFDD technology, as described above, there is an equally strong need to characterize its benefits. Different AFDD tools should be deployed in different types of buildings in different climates, in order to establish the benefits of the tools in terms of energy savings, reduced O\&M costs, and improved conditions for the building occupants.

If significant investments are made in development and initial deployment of AFDD methods and tools, the cost-to-benefit ratio of AFDD implementations for third-party developers, energy service providers, and BAS manufacturers can be expected to become more attractive. There is also a need for market transformation and sponsoring demonstrations to highlight the benefits from use of AFDD tools.

Progress in microelectronics and nano-technology will allow the integration of intelligent sensors and actuators into a distributed open network. Therefore, besides the research work focused on evaluating the existing diagnostic systems, continued research work is necessary in the following areas:

1. Development of standardized diagnostic architectures for retrofitting sensors and diagnostic modules to existing building control system.

2. Development of analytical sensors that use mathematical models plus existing sensor measurements for building controls to estimate the physical variables required for diagnostics.

3. Study of the benefits and capabilities of analytical sensors versus physical sensors. Identify the physical variables that can be estimated with accuracy using mathematical models instead of installing physical sensors.

4. Development of distributed control/diagnostics systems for future buildings equipped with intelligent sensor networks.

5. Development of future distributed building control/diagnostic systems that are modular and scalable allowing applications to buildings of various sizes. This includes research in intelligent sensor/actuator networks for building controls and the optimal distribution of intelligences in the network.

\subsection{Commissioning}

\subsubsection{Background Need}

Three major barriers to the adoption of commissioning are evident. There is not a good understanding of what commissioning is, why it is needed, and the relationship between the costs and benefits.

Second, there is a need for consistent terminology; the term "commissioning” is becoming widely used within the buildings industry, yet "best practices" are not widely understood. Third, there is a need for development and testing of commissioning tools to assist in managing data and documenting the intensive activity involved. 
Because of the many organizations involved, it is necessary to achieve a common understanding of commissioning and the processes involved through a harmonization of terminology. There are several types of commissioning for which there are many different definitions and associated tasks. The main types of commissioning are:

- Initial Commissioning or new building commissioning - commissioning applied to production of a new building and/or installation of new systems,

- Retro-Commissioning - first-time commissioning implemented in an existing building in which a documented commissioning process was not implemented before

- Re-Commissioning - commissioning implemented after the initial commissioning or the retrocommissioning when the owner hopes to verify, improve, and document the performance of building systems.

- On-Going Commissioning (or Continuous Commissioning) - commissioning conducted continually for the purposes of maintaining, improving, and optimizing the performance of building systems after the initial commissioning or the retro-commissioning. This activity is closely related to continuous performance monitoring and fault detection and diagnostics.

In an effort to harmonize terminology and advance tool development, the IEA's Annex 40 is, aimed at developing, validating, and documenting tools for commissioning buildings and building services. In addition to establishing guidelines on commissioning procedures and recommendations for improving the commissioning process, the Annex is developing prototype software that can be implemented in standalone tools or embedded in energy management and control systems.

With the variety of existing and emerging technology, the US buildings industry needs to understand the techniques to support building commissioning and bring advanced technology to use to minimize the costs of commissioning and maximize the benefit. Building commissioning has both institutional and technological issues. The institutional issues include the need for building owners to understand what commissioning is and to support the development of cost-effective commissioning processes through careful review of the techniques and benefits of commissioning. The technological questions include

- What is the best technical method to test each type of component or system?

- How do you develop a sample design to avoid testing every component in a large building?

- How do you record results of commissioning tests for later use?

- How can the EMCS be best used during commissioning, which EMCS technologies best support commissioning?

- How can technology be best used to automate commissioning tests?

- How can simulation be used to augment commissioning tests?

- How can technology help ensure that items identified and corrected during the commissioning process remain in proper operational order?

\subsubsection{Current Status}

Research on commissioning can be divided into seven areas:

- $\quad$ new and existing building commissioning test methods

- continuous commissioning and performance monitoring 
- automated methods (self commissioning, adaptive, and automated)

- life-cycle information integration

- persistence of savings

- market issues.

\subsubsection{New Test Methods for Building Commissioning}

A Functional Test Guide is being developed by PECI and LBNL, funded by DOE and CEC, to help educate commissioning engineers (Haasl et al. 2002). This R\&D is intended to 1) lower the costs for commissioning by disseminating standard test protocols, 2) provide the knowledge and background for others to develop custom tests, and 3) help increase commissioning activities by defining standard public practices that are widely available for common use. There is little research underway into existing building retro-commissioning intervention methods, although such research is greatly needed. The Functional test guide provides some material for commissioning test plan developers in existing buildings. There is a need for interfacing these tests with EMCS. The unique protocols and configurations in EMCS complicate the use of the EMCS in testing.

On-going commissioning and performance monitoring - There is a need to improve the link between commissioning and performance monitoring. See the Performance Monitoring sections of this document.

\subsubsection{Automated Methods and Tools}

Since buildings are integrated systems, automated commissioning and retuning/ recalibrating building set points requires a system-level approach. System-wide design of experiments to identify the building system allows commissioning and retuning using minimal test time and effort. The developed techniques may be used interchangeably to reduce the complexity of manual tuning of fault detection algorithms, thereby facilitating entry of such technologies into the market place.

\subsubsection{Life-Cycle Information Integration}

Building commissioning provides a unique place to link design and operations. It is critical to build data schema, commissioning techniques, and data management systems that bridge the gap between design models and ongoing operations. LBNL and others in the International Alliance for Interoperability are conducting research into HVAC schema for the Industrial Foundation Classes in order to provide such a framework. DOE and CEC fund this work. Similarly, ASHRAE (TC 1.5 Computer Applications) recently sponsored a project in this area: Project 1032 - Identification and Preservation of Building Design Information for Use in Commissioning and Operations.

\subsubsection{Persistence of Savings}

Both new construction and retro-commissioning interventions involve bringing expert engineers to a building to test building systems and identify opportunities for improving operations. The question arises, however, as to how long these savings persist. PECI, Texas A\&M, and LBNL are involved in research projects to address this question, funded by CEC, DOE, and CIEE, and the Sacramento Municipal Utility District. 


\subsubsection{Market Issues}

Market research is needed to understand how to move commissioning into wide spread practice. Several market research activities have been conducted including PECI's study of the market for commissioning in California and the evaluation of the Northwest Public Buildings commissioning programs (see www.nwalliance.org/resources/reports/107.pdf). The California Commissioning Collaborative (or the CCC, described at www.cacx.org) is an ad hoc group of government, utility, and building services professionals who are committed to developing and promoting viable building commissioning practices in California. The CCC has developed a case study database protocol to help collect future commissioning case study data in a standard format. Consistent case study data are needed to help evaluate the cost effectiveness of commissioning.

In addition to $R \& D$, it is also important to enhance the use of advanced technology and advanced commissioning methods, tools, and procedures in current commissioning educational programs. This is important because these educational programs play a key role in the deployment of commissioning concepts. Examples of current educational programs include

- University of Wisconsin -Total Building Commissioning and Commissioning of HVAC Systems and Other Building Components

- ASHRAE - An Integrated Approach to Building Commissioning

- U.S. Army Corps of Engineers - PROSPECT (for federal employees) -Mechanical Systems Commissioning and Commissioning Workshops.

- DOE Federal Energy Management Program's Continuous Commissioning Guidebook for Federal Managers, October 2002 http://www.eere.energy.gov/femp/operations_maintenance/commissioning_guidebook.cfm

During 1998 the US DOE supported the development of a National Strategy for Building Commissioning that focused on how to build the market (PECI 1998; DOE and PECI 1998). The study recommended that the DOE work in partnership with state governments, utilities, existing professional associations, and public-private oversight.

\subsubsection{Proposed Research}

Below is a summary of key areas for additional commissioning R\&D.

1. Improve understanding of the costs and benefits of commissioning - One common question after "what is it?" is "what are the costs and benefits of commissioning?” Issues include reviewing which costs are considered in the costs for commissioning, evaluating the merits of different benefits, which costs are avoided as a result of commissioning. Develop a research plan to work with the building industry to track cost-benefit data on deployment of commissioning techniques at industry scales. Cost-benefit analysis should also be used to reduce the costs of commissioning and to provide feedback to the buildings industry on advancements in buildings technology that reduce such costs. Advanced commissioning procedures are needed to achieve such cost reductions. Significant results can be expected in the near term, although this should be an ongoing effort that tracks the impact of improvements in commissioning practice.

2. Develop robust commissioning design methodologies - Commissioning is fragile and the benefits decay over a few years as remodeling and undiagnosed faults appear. Develop and use whole 
building models to explore the impacts of potential building changes on the energy efficiency associated with possible commissioning approaches.

3. Develop advanced commissioning test methods - There is a lack of detailed knowledge of how to test many types of HVAC systems. Research is needed to help bridge the gap between fault detection and diagnostic research and conventional commissioning. This research should address the type of measurements needed, the adequacy of existing sensors, and the need for new sensors (especially with air flow monitoring), over what period should the measurements be taken, etc. This could be completed in the near to mid term.

4. Linking commissioning and continuous performance monitoring - Support research to understand how to link one-time or short-term commissioning interventions with continuous performance monitoring. Standard methods for continuous performance monitoring systems should be directly linked to the commission tests. It is critical that energy and other savings from commissioning persist over time to be cost effective; however, without improvements in performance monitoring, persistence of savings is unknown. Demonstrate a continuous commissioning strategy and validate the expected energy savings. This could be completed in the near to mid term.

5. Energy analysis and commissioning tests - The majority of commissioning test methods evaluate the functionality of control and HVAC systems. Support the development of additional tests to help evaluate the energy performance of building systems for both new and existing buildings. These tests should be linked to energy models that help predict the energy savings associated with a change in the building systems. This could be completed in the near to mid term.

6. Information management systems and commissioning - Support research to help improve information management in commercial buildings and ensure that such improvements include organizing data for commissioning. This activity includes automatically linking design data with commissioning tests, updating design data as a result of commissioning tests, and better linking operations data with commissioning tests. This could be completed in the mid term.

7. Self commissioning HVAC systems with autonomous optimization.

8. Apply and demonstrate system identification techniques to automate initialization for commissioning.

9. Apply and demonstrate experimental design techniques to improve the efficiency of the commissioning process.

\subsection{Optimal Control}

\subsubsection{Background Need}

In principle, the control of any energy-consuming system can be optimized. In practice, in the great majority of buildings, both HVAC and lighting systems are controlled using non-optimal, heuristic strategies. The benefits of using some form of optimized control in buildings are not well established, but the energy savings resulting from substituting control strategies based on optimization for conventional control strategies in conventional HVAC systems appears to be relatively modest ( $\sim 5-10 \%$ - Brandemuehl 1998). When the cost of energy varies significantly with the time of day, the potential cost savings resulting from optimization of the control can be significantly greater, ranging from $6 \%$ to $30 \%$ (KintnerMeyer and Emery 1995; Henze 2003), depending on:

- the amount of exposed thermal mass

- whether there is an active thermal storage system 
- the variation in the electricity price and the ambient conditions between day and night.

As systems become more complex, e.g. the substitution of variable speed equipment for fixed speed equipment or the addition of on-site electricity generation equipment, the potential benefits of optimized control increase. As the benefits increase, the return on investment from implementing optimized control, or some approximation thereto, in order to reduce operating costs, also increases.

Other industries, including the process industries, make much more extensive use of control optimization than does the buildings industry. In a survey paper, Qin and Badgwell (2003) report that model-based predictive control is used in more than 4,000 industrial applications. It is typically implemented as supervisory control to determine control set points that will be regulated by other controllers, such as a PID controller. Implementation of control optimization methods in buildings involves a unique set of challenges; one example is the wide range of operating points encountered in HVAC systems, which mitigates against the use of linear models as local approximations to the nonlinear characteristics of a number of types of HVAC equipment.

Other reasons for the greater adoption of optimal control in other industries include better-defined control objectives and greater sensitivity of operating costs to control performance. (The major cost in most buildings is salaries but an objectively derived link between indoor environmental conditions and productivity remains elusive.) Related factors are the fragmented nature of the buildings industry and the relatively low skill level of those who install and operate HVAC and other energy-consuming systems in buildings. These differences suggest that, while it may be possible to take control optimization methods developed for other industries and apply them in buildings, the way in which they have to be applied is likely to be uniquely different to the way they are applied in other industries, hence the need for R\&D in control optimization for buildings.

One of the major impediments to the adoption of optimal control strategies for buildings operation is the difficulty with which accurate cost/performance characteristics of installed HVAC equipment can be obtained for input to optimization algorithms. The buildings operation industry requires low-cost and easily implementable control strategies with little set-up time. This requires automated system identification methods to determine the characteristics of the installed equipment.

\subsubsection{Current Status}

Energy-consuming systems in buildings, such as HVAC, lighting, and vertical transportation, can be divided into two categories: those whose operation can be considered to be completely determined by current boundary conditions and those whose operation depends in part on the consequences of previous operation, e.g., thermal storage systems. Control of the first type is a static optimization problem whereas control of the latter is a dynamic optimization problem. The operation cost of building energy systems that use thermal storage systems (such as the building's thermal capacitance or ice tanks) or on-site power generation (such as fuel-cells) depends on the proper scheduling of the component operation. The proper utilization of these storage systems can significantly reduce the HVAC first cost and operating cost, and shift peak energy demand. Since the utilization of these storage systems is a dynamic process, its proper management may be improved if the expected cooling load, heating load, and energy rate structure over a certain time horizon, say one day, can be predicted with sufficient accuracy. 
There has been a significant amount of work over the last fifteen years, much of it at the University of Wisconsin, on the optimization of the control of HVAC systems, starting with the work of Braun et al. (1989) on the static optimization of the control of chilled water plants without storage. More recently, interest has shifted to the dynamic optimization problems associated with the control of HVAC systems with thermal storage, e.g. Kintner-Meyer and Emery (1995), Henze (2003), Henze et al. (2003).

In the process industries, the development of model-based predictive control methods has now reached a relatively mature stage (Mayne et al. 2000). Future research is expected to focus on the development of robust methods, i.e., methods that maintain properties such as stability and performance in the presence of uncertainty.

There is a strong link between optimal control and diagnostics in that faults in a controlled system can significantly reduce the benefits of optimal control by producing a mismatch between the actual behavior of the system and the behavior predicted by the models used by the optimal control procedure. It is also possible to use the same models for both model-based fault detection and optimal control.

\subsubsection{Proposed Research}

Research should focus on the following questions:

1. What are the potential energy savings, peak demand reductions, and cost savings from the use of optimal control instead of conventional control for different classes of HVAC systems in different climates? For which parts of an HVAC system should optimal control strategies be used and for which parts should conventional control strategies be used? Simulation, based on an extended version of EnergyPlus, could be used to conduct a comprehensive assessment that could be completed in the near to mid term. Verification of the simulation predictions using measured results from implementations in real buildings could be completed in the mid term.

2. Which optimal control methods are best suited to on-line implementation in buildings? How robust are these methods in their current form, i.e., how well do they perform in the presence of uncertainty, disturbances, model mismatch, or degradation of HVAC component performance? How much effort does it take to configure the control system? What advantages do methods based on artificial intelligence, such as evolutionary programming, appear to have that make them worthy of further study? A paper study could be completed in the near term.

3. How can on-line optimal control procedures be made more robust, easy to configure, and more efficient computationally? Development of improved methods and field testing of these methods could be completed in the mid term.

4. To what extent can improved heuristic control strategies approximate the performance of optimal control strategies? How much simpler, more robust, easier to implement, and easier to maintain are these strategies than the corresponding optimal strategies? A simulation-based assessment could be completed in the near to mid term; verification of key findings using real buildings could be completed in the mid term.

5. Can model-based optimal control strategies be configured automatically using information generated during the design phase and verified during commissioning? Optimal control procedures based on EnergyPlus could be developed in the near to mid term and field tested in the mid term. Development of optimal control procedures that can be configured automatically from a persistent data model using Industry Foundation Classes (IFC) could be completed in the mid term and demonstrated in the mid to long term. 
6. What are the benefits of replacing models based on manufacturers' performance data with models based on installed performance characteristics identified in the field? If the benefits are significant, are existing system identification techniques suitably accurate and robust or is further development work required? These questions could be addressed in the mid term.

7. Is there an advantage to integrating model-based optimal control strategies with model-based fault detection and diagnosis procedures? This question could be addressed in the near to mid term.

8. Can climate and weather data be used effectively to adaptively tune control systems to achieve optimal energy savings?

9. What are the advantages of an automatic HVAC reconfiguration and commissioning system?

10. What are the advantages of integrating whole building energy systems? Can peak loads be shaved to save energy, lower costs, and lower grid demand? Can targeted personal comfort be achieved at greater energy efficiencies? Can overall building energy demand be managed more effectively by integrating lighting loads with HVAC loads? How can external information such as the weather forecasts or power grid load be used to optimize building performance? Can integrated whole building systems enable better diagnostics and prognostics that lead to improved service and logistics for building operators?

\subsection{Development Environments and Design Tools}

This section is concerned with several enabling and supporting facilities that are, or would be, based on common underlying technologies:

- development environments for research and product development and testing

- design tools to support the generation of control strategies for specific buildings

- simulators for educating engineers and training technicians and sales people.

Each one of these facilities addresses a different need. In the first case, control system vendors would benefit from the availability of tools that provide better support for each stage of product development, from the initial exploration of new ideas to the last stages of testing before field trials. Current support tools, even those used by the majority of the larger controls vendors, are relatively simplistic and are not well suited to the more advanced or demanding applications, particularly those that involve more than a single control loop. This assessment is based on personal observation and informal discussions with people in the industry. An enhanced development environment could either be used by controls vendors or other commercial organizations or by research organizations developing new methods for control or fault detection and diagnosis that could be adopted by private industry.

In the second case, whereas the controls vendors have software tools to support the generation of control programs for their own hardware and firmware from sequences of operation and their libraries of standard strategies for common applications, there are no tools to support the mechanical engineer in the design of efficient sequences of operation for even slightly unconventional applications, such as a chilled water plant with multiple variable speed chillers. Currently building simulation tools used in HVAC design, such as DOE-2, TRACE, and HAP, have very limited capabilities to assess different control strategies, even at the supervisory control level. Furthermore, simulations assume that controls are perfect, in some ways, limiting the amount of credit that can be given to optimization. Lack of suitable tools is a barrier, though not the only one, to the more widespread use of more energy-efficient control strategies. In the 
first instance, these tools would be used by high end A\&E firms and by specialist consultants in the design of innovative buildings and systems or systems with special requirements for close control, enhanced resistance to toxic agents, demand responsiveness etc. In due course, these methods, and the necessary customization of the control system, would become more widely adopted and the design tools would evolve to be more easily used by a broader range of design engineers.

In the third case, there are no simulators or other educational tools specifically adapted for HVAC available for either academic or vocational college programs. Controls vendors make some use of the simple simulators used to exercise control equipment for training purposes, but these simulators have limited capabilities, typically using manually operated potentiometers to provide inputs to the controllers. As the need increases for engineers, technicians and building operators to have better knowledge of control theory and HVAC control technology, simulation-based education and training tools will be more widely used in universities, vocational schools, and industry.

\subsubsection{Current Status}

Over the last 20 years, NIST has developed "emulators" for hardware-in-the-loop testing of simple control systems. An emulator is a tool that combines real and simulated components to reproduce the performance of an entire building in the laboratory. The real components are typically the various automation and control systems and the simulated components are the building envelope and mechanical systems. An emulator provides a way to examine the interactions of the various systems and to see how the building reacts under adverse events, such as equipment failure or a fire.

NIST’s current building system emulator, the Virtual Cybernetic Building Testbed (VCBT), is a collection of computer simulations distributed over several computers that are coupled to each other and to commercial building controllers. The controllers are linked to the simulations using a commercial data acquisition system that converts simulated values such as temperatures, pressures, and flows into voltage or current signals that are wired to the controller inputs. The control signals are digitized by the data acquisition system and fed back to the simulations. The overall effect is that the controllers see data that looks like sensor information from real building systems and the simulations respond to the control actions taken by the controllers. Just as a flight simulator simulates an airplane in real time, the VCBT simulates a building, the weather, the HVAC system, and the heating/cooling plant in real time (Bushby et al. 2001). The VCBT design details are based on experience gained from previous building system emulator research (Kelly et al. 1991).

The VCBT has been used to test Air Handling Unit Performance Assessment Rules (APAR) and VAV Box Performance Assessment Control Charts (VPACC), two fault detection and diagnostic (FDD) tools developed by NIST (Castro et al. 2003). The VCBT has also been used to conduct a blind test of the Whole Building Diagnostician (WBD), an FDD tool developed by PNNL (results to be published soon).

A number of buildings research organizations around the world that participated in IEA Annex 17 have also studied building system emulation. Of these, only CSTB in France has continued to develop and apply emulators for HVAC control applications.

Other relevant work includes the development of the SPARK and EnergyPlus simulation tools at LBNL. SPARK is a numerically efficient tool for simulating non-linear, dynamic systems such as HVAC systems. It is currently being used to model HVAC systems and their controls and to simulate their 
behavior on time-scales down to one second. It is also being used as the basis of a "hardware-in-theloop” emulator/tester currently under development at LBNL. SPARK is currently being integrated with EnergyPlus, which will allow the control of whole buildings to be simulated more easily.

ASHRAE sponsored a research project (825-RP) to develop a simulation testbed for control algorithms and strategies (Haves et al. 1998). A set of models of VAV system components (fans, coils, terminal boxes, ducts, duct fittings, valves, dampers, actuators and sensors) were developed for the modular simulation programs HVACSIM+ and TRNSYS. A framework for modeling digital control systems in these two programs was also developed, including a library of generic control functions typical of those found in EMCS's. An example application was produced, based on a real building and consisting of a VAV air handling unit and six zones. The example was used to illustrate the simulation of the interactions between different control loops, including the supply fan static pressure loop and the flow control loops in pressure-independent VAV boxes.

If simulation or emulation is to be used in the development and testing of fault detection and diagnosis methods and tools, it is necessary to be able to simulate faulty operation as well as correct operation. This typically requires detailed, explicit modeling of the operation of the equipment and the controls, since faulty operation can be different enough from correct operation to invalidate the assumptions and idealizations that are implicit in most building simulation programs. A set of AHU component models for HVACSIM+ that treat some common faults was developed at Oxford University to support the testing of an automated commissioning tool (Haves 1997).

\subsubsection{Proposed Research}

In the future, the role of environments such as NIST's VCBT and the virtual building controls laboratory under development at LBNL will be to serve as a platform for the development of other advanced control technologies. Tests can be conducted under reproducible, carefully controlled conditions, including weather, without endangering the comfort or safety of occupants in a real building. These testing laboratories provide a means to test new concepts for control strategies and prototype products in a way that is economical, efficient, and convenient.

Due to specific known limitations of the VCBT, such as the difficulties associated with changing the building model or updating the simulation model, there is a need for a more flexible development environment. EnergyPlus coupled to SPARK is a viable candidate for further development as the simulation engine for this new development environment, which could be a collaborative effort involving LBNL and NIST. SPARK+EnergyPlus could also serve as the simulation engine for the design tool and the training tool described in the Technology Need section. The only other obvious starting point for such environments and tools is MATLAB/SIMULINK, which has the advantage of being widely used in different industries but has the disadvantage of being a proprietary product and not being specifically adapted for application to buildings.

The next steps should be to specify the requirements for the different applications and identify the development work needed, which is likely to include

1. Development of models of HVAC equipment and control system hardware and firmware (near term). These tools could be used to determine component sizing and system robustness as well as to assess strategies for control or demand response. 
2. Development of occupancy and density models to enable development of new algorithms for optimizing building operations and minimizing energy consumption.

3. Development of user interfaces for the different applications (near to mid term)

4. Development of both analog and digital interfaces to real control systems (near term)

5. Development of data models for control systems and strategies for incorporation in the International Alliance for Interoperability's IFC data model for buildings (near to mid term).

6. Development of integrated, whole building models to explore the impacts of alternative energy use or management technologies (i.e., HVAC or lighting), system configurations, and integrated supervisory control strategies on buildings and to provide building energy efficiency and system robustness assessments. Whole building models will also enable the development of diagnostic tools, integrated control strategies, and rapid prototyping.

7. Analysis of a wide variety of buildings, climates, and local energy costs using whole building models to derive operational "design rules" that are scalable and adaptable across building types.

8. Development of building operator decision support systems based on whole building model analysis.

9. Development of simulation training tools for HVAC installers, consulting engineers, and building operators.

10. Analysis and assessment of integrated building system energy management strategies using whole building models. For example, evaluate the benefits of managing lighting according to knowledge of occupancy or using a strategy that modifies the speed of the elevator based on daily energy demand and costs.

11. Demonstration and validation of system models.

One option would be to develop a National User Facility for controls and diagnostics development and testing. The facility could be used by companies for product development and could also be used for product testing and certification. The facility would be accessible physically at one or more laboratories and would also be accessible via the Internet. The specification for the tools used in the facility would be published so that other organizations, private and public, could construct similar facilities and expect to obtain reproducible results. Operating experience and enhancements to the software tools would be shared among a group of cooperating users. 


\subsection{REFERENCES}

ACHRN. 2002. "Medical Center Improves the Health of Its BAS,” ACHR News, 9 December, p. 18.

ACHRN. 2003. “Focus: Indoor Air Quality,” ACHR News, 17 March.

ADL. 1997. Massachusetts Market Transformation Scoping Study: Stage II Final Report, Arthur D. Little, Inc. for the Massachusetts Gas DSM/Market Transformation Collaborative, September.

ANSI/ASHRAE. Standard 135-2001. BACnet ${ }^{\circledR}$ A Data Communication Protocol for Building Automation and Control Networks, American Society of Heating Refrigerating and AirConditioning Engineers, Inc. Atlanta, Georgia. www.ashrae.org.

Ardehali, M.M. and T.F. Smith. 2002. Literature Review to Identify Existing Case Studies of ControlsRelated Energy-Inefficiencies in Buildings, Technical Report: ME-TFS-01-007, Department of Mechanical and Industrial Engineering, The University of Iowa.

Ardehali, M.M., T.F. Smith, J.M. House, and C.J. Klaassen. 2003. "Building Energy Use and Control Problems: An Assessment of Case Studies.” Accepted for ASHRAE Transactions, Vol. 109, Pt. 2, 2003.

ASHRAE. 2001. ASHRAE Standard 62-2001: Ventilation for Acceptable Indoor Air Quality, American Society of Heating, Refrigeration and Air-Conditioning Engineers, ASHRAE Press, Atlanta, Georgia.

ASHRAE. 2003. Risk Management Guidance for Health, Safety and Environmental Security Under Extraordinary Incidents. Report of Presidential Ad Hoc Committee for Building Health and Safety under Extraordinary Incidents. American Society of Heating, Refrigerating, and AirConditioning Engineers. Atlanta, Georgia. Available at: http://xp20.ashrae.org/about/extraordinary.pdf .

AUS Consultants. 2001. Impact of a Continuing Electricity Crisis on the California Economy, Moorestown, NJ, May 2001.

Bailey, M.B. 1998. The Design and Viability of a Probabilistic Fault Detection and Diagnosis Method for Vapor Compression Cycle Equipment. Ph.D. Thesis, School of Civil Engineering of University of Colorado, Boulder, Colorado.

Barsoum, R.F., 1995, “Smart Building Operations,” Appearing in Chapter 5 of Direct Digital Control, J.J. McGowan, editor, The Fairmont Press, Englewood Cliffs, NJ.

Barwig, F.E., J.M. House, C.J. Klaassen, M.M. Ardehali, and T.F. Smith. 2002. "The National Building Controls Information Program,” in Proc. ACEEE Summer Study on Energy Efficiency in Buildings. Pacific Grove, CA, August, American Council for an Energy Efficient Economy, Washington D.C.

BCS Partners. 2002. The Building Control Systems Market (2001-2006), Report by BCS Partners, July.

BOMA. 1999. What Office Tenants Want - 1999 BOMA/ULI Office Tenant Survey Report, Report by the Building Owners and Managers Association (BOMA) International and the Urban Land Institute (ULI).

BOMA. 2000. “Integrated Systems: Increasing Building and Workplace Performance,” A Professional Paper from the BOMA International Foundation. Available at: http://www.boma.org/download/research/whitepages.pdf . 
Brambley, M.R., R.G. Pratt, D.P. Chassin, and S. Katipamula. 1998. “Automated Diagnostics for Outdoor Air Ventilation and Economizers.” ASHRAE Journal, Vol. 40, No. 10, pp. 49-55, October 1998.

Brambley, M.R., R. Briggs, S. Katipamula, C. Dasher, L. Luskay, and L. Irvine. 2002. "Investigating Strategies for Automating Commissioning." 10th National Conference on Building Commissioning Conference Proceedings. May 8-10, 2002. Portland Energy Conservation Inc. Portland, Oregon.

Brandemuehl, M.J. 1998. Implementation of On-Line Optimal Supervisory Control of Cooling Plants without Storage. Final Report on ASHRAE Research Project 823.

Brandemuehl, M.J. and J.E. Braun. 1999. "The Impact of Demand-Controlled and Economizer Ventilation Strategies on Energy Use in Buildings,” ASHRAE Transactions, vol. 105, part 2, Available at: http://hbctechlit.honeywell.com/techlit/pdf/63-0000s/63-7063.pdf .

Braun JE., SA Klein, WA Beckman, and JW Mitchell. 1989. "Methodologies for optimal control of chilled water systems without storage.” ASHRAE Trans. Vol. 95, part 1, pp. 652-662.

Braun, J.E. 1999. “Automated Fault Detection and Diagnostics for the HVAC\&R Industry.” HVAC\&R Research, Vol. 5, No. 2, pp. 85-86.

Braun, J.E. 1990. "Reducing energy costs and peak electrical demand through optimal control of building thermal storage,” ASHRAE Transaction, 96(2):876-888.

Braun, J.E., KW Montgomery, and N. Chaturvedi. 2001. "Evaluating the Performance of Building Thermal Mass Control Strategies.” Intl. J. HVAC \& R Research, Vol. 7, No. 4.

Breuker, M.S. 1997. Evaluation of a Statistical, Rule-Based Fault Detection and Diagnostics Method for Vapor Compression Air Conditioners. Master's Thesis, School of Mechanical Engineering, Purdue University, Purdue, Indiana.

Breuker, M. S. and J.E. Braun. 1999a. "Common Faults and Their Impacts for Rooftop Air Conditioners.” International Journal of Heating, Ventilating, and Air Conditioning and Refrigerating Research, Vol. 4, No. 2, pp. 303-318.

Breuker, M. S. and J.E. Braun. 1999b. "Evaluating the Performance of a Fault Detection and Diagnostic System for Vapor Compression Equipment.” International Journal of Heating, Ventilating, and Air Conditioning and Refrigerating Research, Vol. 4, No. 4, pp. 401-425.

Brown, R.E. and J.G. Koomey. 2002. Electricity Use in California: Past Trends and Present Usage Patterns, Lawrence Berkeley National Laboratory Report, Draft, LBN:-47992, May. Available at: http://enduse.lbl.gov/Info/LBNL-47992_draft.pdf .

Brown, T.C. 1998. “Fire Protection System Integration,” Security Technology \& Design, August.

BTS. 2002. 2002 Buildings Energy Databook, U.S. Department of Energy, Office of Energy Efficiency and Renewable Energy, 26 July.

Buildings. 2002. "Strategic Security,” Buildings, August. Available at: http://www.buildings.com/Articles/detail.asp?ArticleID=968 .

Buildings.com, 2003, "Start Saving Energy - Operation: Energy Management (1 of 4), How Today’s Healthcare Facilities are Dealing with Energy-efficiency Issues,” Buildings, March. Available at: www.buildings.com (last accessed on 3/13/03).

Bushby, S. 2001. "Integrating Fire Alarm Systems with Building Automation and Control Systems,” Fire Protection Engineering, Summer, pp. 5-11. Available at: http://www.bacnet.org/Bibliography/FPE-7-01.pdf . 
Bushby, S.T. and H.M. Newman. 2002. “BACnet Today,” ASHRAE Journal, October, pp. 10-18.

Bushby, S.T., N.S. Castro, M.A. Galler, C. Park, J.M. House. 2001, Using the Virtual Cybernetic Building Testbed and FDD Test Shell for FDD Tool Development, NISTIR 6818, National Institute for Standards and Technology, Washington DC.

CABA. 2002. Technology Roadmap for Intelligent Buildings, Prepared by the Continental Automated Buildings Association for the Natural Research Council, Canada.

Camacho, E.F. and C. Bordons. 1999. Model Predictive Control, Springer-Verlag, London.

Carter, D.J. and T.A. Moore. 2002. “Occupant Controlled Office Lighting: Design Strategies for Energy Saving,” Proc. Right Light 5, pp. 247-254, Nice, France, May.

Castro, N.S., J. Schein, C. Park, M.A. Galler, S.T. Bushby, and J.M. House. 2003. Results from Simulation and Laboratory Testing of Air Handling Unit and Variable Air Volume Box Diagnostic Tools, NISTIR 6964, National Institute for Standards and Technology, Washington DC.

CBECS. 1999. “Commercial Buildings Energy Consumption Survey (CBECS),” U.S. Department of Energy, Energy Information Administration. Available at: http://www.eia.doe.gov/emeu/cbecs/contents.html .

Chassin DP. 2003. "Power Market Dynamics: The Statistical Mechanics of Transaction-Based Control." In Proceedings of ECOS 2003. PNNL-SA-37929, Pacific Northwest National Laboratory, Richland, Washington.

Checket-Hanks, B. 2003. "Placebo Stats,” ACH\&R News, 31 March, 2003, p. 1, 7-9.

Claridge, D., M. Liu, and W.D. Turner. 1999. "Whole Building Diagnostics,” in Proceedings of the Diagnostics for Commercial Buildings: Research to Practice Workshop, held June 16th \& 17th, 1999 , Pacific Energy Center, San Francisco, Lawrence Berkeley National Laboratory. Available at: http://poet.lbl.gov/diagworkshop/proceedings/ .

Claridge, D.E. 1998. "A Perspective of Methods for Analysis of Measured Energy Data from Commercial Buildings." Special issue on Conservation and Solar Buildings, ASME Journal of Solar Energy Engineering, Vol. 20, No. 3, pp. 150-155.

Claridge, D.E., C.H. Culp, M. Liu, S. Deng, W.D. Turner, and J.S. Haberl. 2000. "Campus-Wide Continuous Commissioning ${ }^{\text {SM }}$ of University Buildings.” In Proceedings of the 2000 ACEEE Summer Study. Pacific Grove, CA, August, American Council for an Energy Efficient Economy, Washington D.C.

Claridge, D.E., J.S. Haberl, M. Liu, J. Houcek, and A. Athar. 1994. “Can You Achieve 150\% Predicted Retrofit Savings: Is It Time for Recommissioning?” In Proceedings of the 1994 ACEEE Summer Study. Pacific Grove, CA, August, American Council for an Energy Efficient Economy, Washington D.C.

Claridge, D.E., M. Liu, Y. Zhu, M. Abbas, A. Athar, and J.S. Haberl. 1996. "Implementation of Continuous Commissioning in the Texas LoanSTAR Program: Can You Achieve 150\% Estimated Retrofit Savings Revisited.” In Proceedings of the 1996 ACEEE Summer Study. Pacific Grove, CA, August, American Council for an Energy Efficient Economy, Washington D.C.

Cler, G. et al. 1997. Commercial Space Cooling and Air Handling: Technology Atlas. E Source, Inc.

Cosiol, J. 2001. "Preventative Maintenance: Tools and Parameters,” Networked Controls, supplement to HPAC Engineering, May, pp. 44-50. 
CSI. 2002. "CSI Approves Concept for Revising MasterFormat ${ }^{\mathrm{TM}}$; New Divisions to Address Expanded Industry Needs; Revisions Designed to Minimize Change for Current Users,” Construction Specification Institute Press Release, 27 February. Available at: http://www.csinet.org/press/pr22702.htm.

Daisey, J.M. and W.J. Angell. 1998. A Survey and Critical Review of the Literature on Indoor Air Quality, Ventilation and Health Symptoms in Schools. LBNL-41517, Lawrence Berkeley National Laboratory, Berkeley, California.

Dalton T., R.J. Patton, and P.J.H. Miller. 1995. "Methods of Fault Detection for a Centrifugal Pump System." On-Line Fault Detection and Supervision in the Chemical Process Industries, IFAC Workshop, Newcastle Upon Tyne, UK, Pergamon Press, New York.

Deru, M. 2003. Final Project Report for DPD, Inc., Office Building, Lansing, MI. NICH Report No. TP550-33108, National Renewable Energy Laboratory, Golden, Colorado.

Deru, M. and E. Hancock. 2003. Preliminary Energy Analysis of the Pennsylvania Department of Environmental Protection's Cambria Office Building. NICH Report No. TP-550-33106, National Renewable Energy Laboratory, Golden, Colorado.

Dexter A. and Pakanen, J. (Ed.). 2001. International Energy Agency: Demonstrating Automated Fault Detection and Diagnosis in Real Buildings. Technical Research Centre of Finland, Laboratory of Heating and Ventilation, Espoo, Finland.

Dexter, A.L. and M. Benouarets. 1996. "Generic Approach to Identifying Faults in HVAC Plants.” ASHRAE Transactions, Vol. 102, No. 1, pp. 550 - 556.

Dodier, R.H. and JF Kreider. 1999. Detecting Whole Building Energy Problems. ASHRAE Transactions, Vol. 105, No. 1.

Dougan, D.S. and L.A. Damiano, 2003, “The Big Carrots: Productivity and Health,” AutomatedBuildings.com, April. Downloaded from: http://www.automatedbuildings.com/news/apr03/articles/ebtron/ebtron.htm .

Dreessen, 2002, “U.S. ESCO Market,” Proc. $2^{\text {nd }}$ International Conference on Improving Electricity Efficiency in Commercial Buildings, 27-29 May, Nice, France.

Energy Design Resources. 1998. "Design Brief: Energy Management Systems”, Design Brief Prepared for Energy Design Resources by E-Source, Inc. Available at: http://www.energydesignresources.com/publications/design briefs/db energymgmt.html.

Energy Design Resources. 2000. “Design Brief: Lighting Controls,” Design Brief Prepared for Energy Design Resources by E-Source, Inc. Available at: http://www.energydesignresources.com/publications/design_briefs/db_lightcontrol.html .

Energy Design Resources. 2001a. “Design Brief: Economizers,” Design Brief Prepared for Energy Design Resources by E-Source, Inc. Available at: http://www.energydesignresources.com/publications/design briefs/dbv2 economizers.html .

Energy Design Resources. 2001b . "Design Brief: Smart Buildings,” Design Brief Prepared for Energy Design Resources by Architectural Energy Corporation. Available at:

EIA. 2001. Annual Energy Review, DOE/EIA-0384, 2001, U.S. Department of Energy, Energy Information Administration, Washington DC.

\footnotetext{
${ }^{1}$ Precise year unclear; 2001 estimated from paper contents (references).
} 
EIA. 2002a. “1999 Commercial Building Energy Consumption and Expenditures (CBECS),” Public Use Data, Energy Information Administration, U.S. Department of Energy, Micro-data files on EIA website: http://www.eia.doe.gov/emeu/cbecs/detailed_tables_1999.html

EIA. 2002b. "Electric Power Monthly,” Energy Information Administration, U.S. Department of Energy, July. Available at: http://www.eia.doe.gov/cneaf/electricity/epm/epm_sum.html .

EIA. 2002c. “Natural Gas Monthly,” Energy Information Administration, U.S. Department of Energy, March. Available at: http://www.eia.doe.gov/oil gas/natural gas/data publications/natural gas monthly/ngm.html .

EIA. 2003. Annual Energy Outlook 2003. Report \#: DOE/EIA-0383 (2003), U.S. Department of Energy, Energy Information Administration, Washington DC.

EPA, 2001, “Energy Star Building Manual”, U.S. Environmental Protection Agency, October. Available at: http://www.energystar.gov/index.cfm?c=business.bus_upgrade_manual .

Engineered Systems. 1999. “Issues \& Events - In Efficiency (and Market Forces) We Trust,” Engineered Systems, July, p. 26.

Fasolo, P.S. and D.E. Seborg. 1995. "Monitoring and Fault Detection for an HVAC Control System.” International Journal of Heating, Ventilation, and Air-Conditioning and Refrigeration Research, Vol. 99, Pt. 1, pp. 3-13.

FERC. 2002. "Working Paper on Standardized Transmission Service and Wholesale Electric Market Design” [18 CFR Part 35] (Docket No. RM01-12-000), November 5, 2002.

Fisk, W.J. 2000. "Health and Productivity Gains from Better Indoor Environments and their Relationship with Building Energy Efficiency,” Annual Review of Energy and the Environment, vol. 25, pp. 537-566.

Gemmill, C. 2003. “Load Control Object,” BACnet Committee SSPC 135 document CG-001-5.

Georgescu, C., A. Afshari, and G. Bornard. 1993. “A Model-Based Adaptive Predictor Fault Detection Method Applied to Building Heating, Ventilating, and Air-Conditioning Process.” TOOLDIAG' 93, Organized by Département d’Etudes et de Recherches en Automatique, Toulouse, Cedex, France.

Glass, A.S., P. Gruber, M. Roos, and J. Todtli. 1995. "Qualitative Model-Based Fault Detection in AirHandling Units.” IEEE Control Systems Magazine, Vol. 15, No. 4, pp. 11 - 22.

Goldman, C., M. Kintner-Meyer, and G. Heffner. 2002. Impact of Enabling Technologies on Customer Load Curtailment Performance. PNNL-13844/ LBNL-13844, Pacific Northwest National Laboratory, Richland, Washington.

Gordon, J.M. and K.C. Ng. 1995. "Predictive and Diagnostic Aspects of a Universal Thermodynamic Model for Chillers.” International Journal of Heat and Mass Transfer, Vol. 38, No. 5, pp. 807 818.

Gregerson, J. 1997. Commissioning Existing Buildings. E-Source Report TU-97-3

Grimmelius, H.T., J.K. Woud, and G. Been. 1995. “On-line Failure Diagnosis for Compression Refrigerant Plants.” International Journal of Refrigeration, Vol. 18, No. 1, pp. $31-41$.

Gu, L. and Haves, P. 2001. Guideline for the Operation of Demand-Responsive HVAC Systems, Report for GSA Region IX, Lawrence Berkeley National Laboratory, Berkeley, California.

Guillemin, A. and N. Morel, “An Innovative Lighting Controller Integrated in a Self-Adapting Building Control System,” Energy and Buildings, V.33, 2001, pp 477-487. 
Guttromson RT, DP Chassin, and SE Widergren. 2003. "Residential Energy Resource Models for Distribution Feeder Simulation." In Proceedings of IEEE Power Engineering Society Annual Meeting, July 2003, Toronto, Canada. PNNL-SA-38072, Pacific Northwest National Laboratory, Richland, Washington.

Haasl, T., D. Sellar,s H. Friedman, M.A. Piette, N. Bourassa and K. Gellespie. 2002. "A New Guide for Commissioning Air Handling Systems: Using a Model Functional Test," in Proceedings of the 2002 ACEEE Summer Study. Pacific Grove, CA, August, American Council for an Energy Efficient Economy, Washington D.C.

Haasl, T., A. Potter, L. Irvine, and L. Luskay. 2003. “Retrocommissioning’s Greatest Hits.” Portland Energy Conservation, Inc., Portland, Oregon. http://www.peci.org/papers/rcxhits.pdf.

Hadley, S.W. 2002. “Building Assurance: September 11 and National Security Implications,” White Paper Prepared for the DOE Office of Energy Efficiency and Renewable Energy, Buildings Program, U.S. Department of Energy, 2 April.

Hall, S. 2001. “Is Your BAS Ready for the Future?” Networked Controls 2002 - A Supplement to HPAC Engineering, July, pp. 119-121.

Han, C.Y., Y. Xiao, and C.J. Ruther. 1999. "Fault Detection and Diagnosis of HVAC Systems.” ASHRAE Transactions, Vol. 105, Pt. 1.

Haves, P., T. Salsbury, and J.A. Wright. 1996b. “Condition Monitoring in HVAC Subsystems Using First Principles Models.” ASHRAE Transactions, Vol. 102, Pt. 1, pp. 519 - 527.

Haves, P. 1997. "Fault Modeling in Component-based HVAC Simulation,” Proceedings of Building Simulation '97, Prague, Czech Republic, September 1997.

Haves, P., D.R. Jorgensen, T.I. Salsbury, and A.L. Dexter. 1996a. "Development and Testing of a Prototype Tool for HVAC Control System Commissioning,” ASHRAE Transactions, Vol. 102, Pt $1,1996$.

Haves, P., Norford, L.K., and DeSimone, M. 1998. "A Standard Simulation Testbed for the Evaluation of Control Algorithms and Strategies,” ASHRAE Trans, 104, Pt 1, 1998.

Hayter, S., P. Torcellini, S. Farrar-Nagy et al. 2002. LANL Sustainable Design Guide LANL Publication LA-UR 02-6914, Los Alamos National Laboratory, Los Alamos, NM.

Henze, G.P., M. Krarti, and M.J. Brandemuehl. 2003. "Guidelines for improved performance of ice storage systems,” Energy and Buildings, 35:111-127, 2003.

Henze, G.P. 2003. "Impact of real-time pricing rate uncertainty on the annual performance of cool storage systems,” Energy and Buildings, 35.

Hill, R., H. Kessler, and J. Lannon. 2000. “Increasing Real Estate Value through Retro-Commissioning,” Proceeding of the $8^{\text {th }}$ National Conference on Building Commissioning, May 3-5.

Holmberg, D.G. 2003. BACnet WAN Security Threat Assessment, BACnet committee SSPC 135 document number DGH-001-2, under review for publication as an NIST internal report. National Institute of Standards and Technology, Washington DC.

Holmberg, D.G. 2003. Logical Entity Object, BACnet committee SSPC 135 document DGH-003-2.

House, J.M., W.Y. Lee, and D.R. Shin. 1999. "Classification Techniques for Fault Detection and Diagnosis of an Air-Handling Unit.” ASHRAE Transactions, Vol. 105, Pt. 1.

House, J.M. and Kelly, G.E. 1999. “An Overview of Building Diagnostics” in Diagnostics for Commercial Buildings: Research to Practice, Organized by Pacific Energy Center, San Francisco, CA. 
House, J.M., H. Vaezi-Nejad, and J.M. Whitcomb. 2001. “An Expert Rule Set for Fault Detection in AirHandling Units,” ASHRAE Transactions, Vol. 107, Pt. 1: pp. 858-871.

Hyvärinen, J. and S. Kärki, S. (Ed.). 1996. International Energy Agency Building Optimisation and Fault Diagnosis Source Book. Published by Technical Research Centre of Finland, Laboratory of Heating and Ventilation, Espoo, Finland.

Industrial Wireless Technology for the 21st Century, December 2002. Available at: http://www.eere.energy.gov/industry/sensors_automation/pdfs/wireless_technology.pdf

Office of Energy Efficiency and Renewable Energy IPMVP. 2002. International Performance Measurement \& Verification Protocol: Concepts and Options for Determining Energy and Water Savings Vol. I, March 2002, www.ipmvp.org.

Issermann, R. 1984. "Process Fault Detection Based on Modeling and Estimation Methods - A Survey." Automatica, Vol. 20, No. 4, pp. 387 - 404.

Isermann, R. and P. Ballé. 1997. "Trends in the Application of Model-Based Fault Detection and Diagnosis of Technical Process.” Control Eng. Practice, Vol. 5. No. 5, pp. 709-719.

Isermann, R. and S. Nold. 1988. "Model Based Fault Detection for Centrifugal Pumps and AC Drives.” In $11^{\text {th }}$ IMEKO World Congress. Houston, Texas, pp. 16-21.

Ivanovich, M.G. 2002. “Networked Controls 2002,” Networked Controls 2002 - A Supplement to HPAC Engineering, July, p. 5.

Jennings, J.D., F.M. Rubinstein, D. DiBartolomeo and S. Blanc, 2000, “Comparison of Control Options in Private Offices in an Advanced Lighting Controls Testbed”, J. of the Illuminating Engineering Society of North America, vol. 29, no. 2.

Jiang, Y., J. Li, and X. Yang. 1995. "Fault Direction Space Method for On-Line Fault Detection.” ASHRAE Transactions, Vol. 101, Pt. 2, pp. 219 - 228.

Johnson, S. 2003. “Despite Terrorism Threat, Building Owners Are Wary of Ventilation Fixes,” San Jose Mercury News, 11 March.

Kammerud, R. C., S.L. Blanc, and W.F. Kane. 1996. “The Impact of Real-Time Pricing of Electricity on Energy Use, Energy Cost, and Operation of a Major Hotel,” Proc. ACEEE Summer Study on Energy Efficiency in Buildings. Pacific Grove, CA, August, American Council for an Energy Efficient Economy, Washington D.C.

Kastovich, J.C., R.R. Lawrence, R.R. Hoffman, and C. Pavlak, 1982, Advanced Electric Heat Pump Market and Business Analysis, Final Report prepared by Westinghouse Electric Corporation for Oak Ridge National Laboratory, ORNL/Sub/79-24712/1, April.

Katipamula S., M.R. Brambley, and L. Luskay. 2003a. "Automated Proactive Commissioning of AirHandling Units." PNWD-SA-5929, Battelle-Pacific Northwest Division, Richland, WA.

Katipamula, S., N.N. Bauman, R.G. Pratt, and M.R. Brambley. 2003b. "Energy Efficient and Affordable Small Commercial and Residential Buildings Research Program -- Project 2.4 - Demonstration of the Whole-Building Diagnostician, Task 2.4.1 - Single-Building Operator Demonstration - Online Test." PNWD-3255, Pacific Northwest Laboratory, Richland, Washington.

Katipamula, S., R.G. Pratt, D.P. Chassin, Z.T. Taylor, K. Gowri, and M.R. Brambley. 1999. "Automated Fault Detection and Diagnostics for Outdoor-Air Ventilation Systems and Economizers: Methodology and Results from Field Testing." ASHRAE Transactions, Vol. 105 Pt. 1. 
Katipamula, S., R.G. Pratt, and J. Braun. 2000. "Building Systems Diagnostics and Predictive Maintenance.” CRC Handbook of Heating Ventilation, and Air Conditioning. Ed. Jan F. Kreider CRC Press, New York.

Katipamula, S. and S. Gaines. 2003. "Characterization of Buildings Controls and Energy Efficiency Options Using Commercial Buildings Energy Consumption Survey.” PNWD-3247. February 2003. Pacific Northwest National Laboratory, Richland, WA.

Keeney, K.R. and J.E. Braun 1997. "Application of Building Precooling to Reduce Peak Cooling Requirements,” ASHRAE Transactions, Vol. 103, Pt. 1, pp. 463-469.

Kelly, G. E., C. Park, and J.P. Barnett. 1991. "Using Emulators/Testers for Commissioning EMCS Software, Operator Training, Algorithm Development, and Tuning Local Control Loops,” ASHRAE Trans., Vol. 97, Pt. 1.

Kintner-Meyer, M., and A.F. Emery. 1995. "Optimal Control of an HVAC system using cold storage and building thermal capacitance,” Energy and Buildings, 23:19-31.

Kintner-Meyer, M. and M. Burns. 1999. Utility/Energy Management and Controls System (EMCS) Communication Protocol Requirements, ASHRAE Research Project 1011-RP. American Society of Heating, Refrigerating and Air-Conditioning Engineers.

Kintner-Meyer, M; M. Brambley, T. Carlon, and N. Bauman. 2002. "Wireless Sensors: Technology and Cost-Savings for Commercial Buildings.” 2002 ACEEE Summer Study on Energy Efficiency in Buildings. pp. 7.121, American Council for Energy-Efficient Economy, Washington, D.C.

Kintner-Meyer M, RT Guttromson, DL Oedingen, and S Lang. 2003. Final Report for the Energy Efficient and Affordable Small Commercial and Residential Buildings Research Program Project 3.3 - Smart Load Control and Grid Friendly Appliances . PNNL-14342, Pacific Northwest National Laboratory, Richland, Washington.

Kranz, H.R. and O. Gisler, 2002, "Standardization \& IT Technology Will shape the BACS Industry,” AutomatedBuildings.com, January. Available at: http://www.automatedbuildings.com/news/jan02/art/hk/hk.htm .

Kumamaru, T., T. Utsunomiya, Y. Iwasaki, I. Shoda, and M. Obayashi. 1991. "A Fault Diagnosis Systems for District Heating and Cooling Facilities.” Proceedings of the International Conference on Industrial Electronics, Control, and Instrumentation, Kobe, Japan (IECON '91), pp. $131-136$.

Kumar, S., S. Sinha, T. Kojima, and H. Yoshida. 2001. "Development of Parameter-Based Fault Detection and Diagnosis Technique for Energy Efficient Building Management System,” Energy Conversion \& Management, vol. 42, pp.833-854.

Lee, W.Y., J.M. House, and D.R. Shin. 1997. "Fault Detection of an Air-Handling Unit Using Residual and Recursive Parameter Identification Methods.” ASHRAE Transactions, Vol. 102, Pt. 1, pp. 528-539.

Lee, W.Y., J.M. House, C. Park, and G.E. Kelly. 1996b. "Fault Diagnosis of an Air-Handling Unit Using Artificial Neural Networks.” ASHRAE Transactions, Vol. 102, Pt. 1, pp. 540-549.

Li, X., V. Hossein, and J. Visier. 1996. "Development of a Fault Diagnosis Method for Heating Systems Using Neural Networks.” ASHRAE Transactions, Vol. 102, Pt. 1, pp. 607 - 614.

Li, X., J. Visier, and H. Vaezi-Nejad. 1997. “A Neural Network Prototype for Fault Detection and Diagnosis of Heating Systems.” ASHRAE Transactions, Vol. 103, Pt. 1, pp. 634 - 644.

Lowry, G., 2002, “Factors Affecting the Success of Building Management System Installations.” Building Serv. Eng. Res. Technology, Vol. 23, no. 1, pp. 57-66. 
LRC, 1998, “Four Views on Ideal Controls”, Lighting Futures, Vol. 3, no. 3. Available at: http://www.Irc.rpi.edu/Futures/LF-OfficeLighting/views.htm.

Lunneberg, T. 1999. "When Good Economizers Go Bad.” E Source Report ER-99-14, E Source, Boulder, Colorado.

Lutron, 2003. Product information available at the website: http://www.lutron.com/radiotouch/

Mahajan, S. et al., 1994. Optimizing the Use of Energy Management and Control Systems to Reduce Peak Load and Energy Consumption in Non-Residential Buildings; available from S. Mahajan (916) 278-5600, Sacramento State University, Sacramento, California.

Mayne, D.Q., J.B. Rawlings, C.V. Rao, and P.O.M. Scokaert. 2000. “Constrained Model Predictive Control: Stability and Optimality,” Automatica, 36:789-814, 2000.

McDonald, C. 2003. “Mold Litigation: On the Brink,” ASHRAE Journal, March, p. 60.

McGowan, J.J. 1995. Direct Digital Control, The Fairmont Press: Englewood Cliffs, NJ.

McGowan, J.J. 2003. “Operating Online: A Real-Time System Integration Update,” Engineered Systems, February. Available at: http://www.esmagazine.com/CDA/ArticleInformation/coverstory/BNPCoverStoryItem/0,2500,91 369,00.html.

McKellar, M.G. 1987. Failure Diagnosis for a Household Refrigerator. Master's Thesis, School of Mechanical Engineering, Purdue University, Purdue, Indiana.

Meador R.J. and D.B. Jarrell. 1999. USMC's Twentynine Palms Central Heating Plant Becomes Most Efficient in the Marine Corps - Success Story. PNNL-SA-32209. Pacific Northwest National Laboratory, Richland, Washington.

Mills, E., H. Friedman, Tehesia Powell et al. 2004. The Cost Effectiveness of Commercial Buildings Commissioning: A Meta-Analysis of Energy and Non-Energy Impacts in Existing Buildings and New Construction in the United States. LBNL-56637. Lawrence Berkeley National Laboratory, Berkeley, California.

Morris, F.B., J.E. Braun, and S. Treado. 1994. "Experimental and Simulated Performance of Optimal Control of Building Thermal Storage,” ASHRAE Transactions, 100(1):402-414, 1994.

Motegi, N. and M.A. Piette. 2002. "Web-based Energy Information Systems for Large Commercial Buildings,” 10th Annual National Conference on Building Commissioning. May.

MTI. 1980. “A High Seasonal Performance Factor Gas Heat Pump for the North Central United States,” Appendix Vol. Viii, Business Potential Study, Mechanical Technology, Incorporated.

Murphy, J. 2002. “Using $\mathrm{CO}_{2}$ for Demand-Controlled Ventilation,” Trane Engineers Newsletter, Vol. 31, no. 3.

Murray, R.M., K.J. Astrom, S.P. Boyd, R.W. Brockett, and G. Stien. 2003. "Future Directions of Control in an Information-Rich World.” IEEE Control Systems Magazine, April 2003.

Nadel, S., F. Gordon, and C. Neme. 2000. "Using Targeted Energy Efficiency Programs to Reduce Peak Electrical Demand and Address Electric System Reliability Problems,” ACEEE Report No, U008, November, American Council for an Energy Efficient Economy, Washington D.C

National Energy Policy Development Group. 2001. National Energy Policy, ISBN 0-16-050814-2, May 2001. 
Neenan, B., D. Pratt, P. Cappers, J. Doane, J. Anderson, R. Boisvert, C. Goldman, O. Sezgen, G. Barbose, R. Bharvirkar, M. Kintner-Meyer, S. Shankle, and D. Bates. How and Why Customers Respond to Electricity Price Variability: A Study of NYISO and NYSERDA 2002 PRL Program Performance. PNNL-14220./LBNL-52209. Pacific Northwest National Laboratory, Richland, Washington.

NEMA. 1992. NEMA Guide to Lighting Controls, Report by the National Electrical Manufacturers Association and Lighting Controls Council.

Ngo, D. and A.L. Dexter. 1999. “A Robust Model-Based Approach to Diagnosing Faults in AirHandling Units.” ASHRAE Transactions, Vol. 105, Pt. 1.

Norford, L.K. and R.D. Little. 1993. "Fault Detection and Monitoring in Ventilation Systems.” ASHRAE Transactions, Vol. 99, Pt. 1, pp. 590 - 602.

Norford, L.K., J.A. Wright, R.A. Buswell, D. Luo, C.J. Klaassen, and A. Suby. 2002. "Demonstration of Fault Detection and Diagnosis Methods for Air-Handling Units (ASHRAE 1020-RP),” Intnl Journal of HVAC \& R Research, Vol. 8, No. 1 (January).

Noura, H., C. Aubrun, D. Sauter, and M. Robert. 1993. “A Fault Diagnosis and Reconfiguration Method Applied to Thermal Plant.” TOOLDIAG' 93, Organized by Département d'Etudes et de Recherches en Automatique, Toulouse, Cedex, France.

Oestreicher, Y., M. Bauer, and J-L. Scartezzini. 1996. "Accounting free gains in a non-residential building by means of an optimal stochastic controller,” Energy and Buildings, 24:213-332, 1996.

Oliveira JS, JB Jones-Oliveira, DP Chassin, and CG Bailey. 2003. A Computational Model for the Identification of Critical Pathways in an Electric Power Grid with Distributed Generation. PNNL-SA-38403, Pacific Northwest National Laboratory, Richland, WA.

Pacific Gas and Electric (PG\&E), 2000, “AB 970 Nonresidential Standards Proposals - Codes and Standards Enhancement (CASE) Study: Lighting Controls”, Draft, 17 November. Available at: http://www.energy.ca.gov/title24/associated_documents/2000-11-17_PGE_CASE.PDF .

Pape, F.L.F., J.W. Mitchell, and W.A. Beckman. 1990. "Optimal Control and Fault Detection in Heating, Ventilating, and Air-Conditioning Systems.” ASHRAE Transactions, Vol. 97, Pt 1, pp. $729-736$.

Parks, J. and M. Kellow. 2000. “SMUD’s Retrocommissioning Program,” Proceeding of the $8^{\text {th }}$ National Conference on Building Commissioning, May 3-5.

PECI. 1998. National Strategy for Building Commissioning, Portland Energy Conservation, Inc., Portland, Oregon. www.peci.org/cx/natstrat.html.

Peitsman, H.C and L.L. Soethout. 1997. “ARX Models and Real-Time Model-Based Diagnosis.” ASHRAE Transactions, Vol. 103, Pt. 1, pp. 657 - 671.

Peitsman, H.C. and V. Bakker. 1996. "Application of Black-Box Models to HVAC Systems for Fault Detection.” ASHRAE Transactions, Vol. 102, Pt. 1, pp. 628-640.

Piette, M.A. and B. Nordman. 1996. "Costs and Benefits of Utility Funded Commissioning of EnergyEfficiency Measures in 16 Buildings,” ASHRAE Transactions, Atlanta, GA, Vol. 102, Pt 1. February 1996, LBNL-37832, Lawrence Berkeley National Laboratory, Berkeley, California. 
Piette, M.A., L. Gartland, S. Khalsa, P.Rumsey, E.L.Lee, A. Sebald, and C. Shockman. 1998. "Development and Testing of an Information Monitoring and Diagnostic System for Large Commercial Buildings ,” Proceedings of the 1998 ACEEE Summer Study on Energy Efficiency in Buildings. August, 1998, Pacific Grove, California, American Council for an Energy Efficient Economy, Washington DC.

Piette, M.A., S. Khalsa, and P. Haves. 2000. "Use of an Information Monitoring and Diagnostic System to Improve Building Operations,” Proc. ACEEE Summer Study on Energy Efficiency in Buildings. August, Pacific Grove, California, American Council for an Energy Efficient Economy, Washington DC.

Piette, M.A., S. Kinney, and P. Haves. 2001. "Analysis of an Information Monitoring and Diagnostic System to Improve Building Operations,” Energy and Building, vol. 33, no. 8, pp. 783-791.

Piette, M.A., S. Kinney, N.J., Bourassa, K. Kinney and C. Shockman. 2003. "Early Findings from a Second Generation Information Monitoring and Diagnostic System,” draft paper to be presented at the $11^{\text {th }}$ National Conference on Building Commissioning, May 2003.

Pless, S. and P. Torcellini. 2002. Energy Performance Evaluation of a Low-Energy Academic BuildingAdam Joseph Lewis Center for Environmental Studies, Oberlin, Ohio, NREL Technical Publication NREL/TP 550-33180, National Renewable Energy Laboratory, Golden, Colorado.

Potter, A., H. Friedman, T. Haasl and D.E. Claridge. 2002b. "Persistence of Benefits from New Building Commissioning,” In Proceedings of the 2002 ACEEE Summer Study, pp. 3.129 - 3.140, August, Pacific Grove, California, American Council for an Energy Efficient Economy, Washington DC.

Pratt, R.G., N.N. Bauman, and S. Katipamula. 2003. New Technology Demonstration of the WholeBuilding Diagnostician at the Federal Aviation Administration-Denver Airport. PNNL-14157, Pacific Northwest National Laboratory, Richland, Washington.

Princeton Synergistics. 1995. A Stochastic Market Penetration Model (MPM) for Building Envelope \& End-Use Products, Final Report Prepared by Princeton Synergetics, Inc. for Battelle Pacific Northwest Division, Richland, Washington.

Qin, S. J., and T.A. Badgwell. 2003. "A survey of industrial model predictive control technology,” Control Engineering Practice.

Quantum. 2003. “Commissioning in Public Buildings Project, No. 3,” Final Report Prepared by Quantum Consulting for the Northwest Energy Efficiency Alliance, Report \#E03-107, February. Available at: www.nwalliance.org/resources/reports/107.pdf .

Reed, J.H., A. Oh, D.R. Dohrmann, S. Bender, C. Chappell, and P. Landry. 2002. "Market Segments in the Commercial Remodeling and Renovation Sector,” Proc. ACEEE Summer Study on Energy Efficiency in Buildings, Pacific Grove, California, American Council for an Energy Efficient Economy, Washington DC.

Reed, J.H., A.D. Oh, and N.P. Hall. 2000. “The Structure and Operation of the Commercial Building Market,” Proc. ACEEE Summer Study on Energy Efficiency in Buildings. Pacific Grove, California, American Council for an Energy Efficient Economy, Washington DC.

RLW Analystics. 1999. "Final Report: Non-Residential New Construction Baseline Study,” California State-Level Market Assessment and Evaluation Study, 8 July, RLW Analystics, Middletown, Connecticut.

Rossi, T.M. 1995. Detection, Diagnosis, and Evaluation of Faults in Vapor Compression Cycle Equipment. Ph.D. Thesis, School of Mechanical Engineering, Purdue University, Purdue, Indiana. 
Rossi, T.M. and J.E. Braun. 1996. "Minimizing Operating Costs of Vapor Compression Equipment with Optimal Service Scheduling.” International Journal of Heating, Ventilating, and Air Conditioning and Refrigerating Research, Vol. 2, No. 1, pp. 3-26.

Rossi, T.M. and J.E. Braun. 1997. "A Statistical, Rule-Based Fault Detection and Diagnostic Method for Vapor Compression Air Conditioners.” International Journal of Heating, Ventilating, and Air Conditioning and Refrigerating Research, Vol. 3, No. 1 pp. 19-37.

Roth, K.W., D. Westphalen, J. Dieckmann, S.D. Hamilton, and W. Goetzler. 2002. “Energy Consumption Characteristics of Commercial Building HVAC Systems - Volume III: Energy Savings Potential,” Final Report by TIAX LLC to US Department of Energy, Office of Building Technology, State and Community Programs, July. Available at: http://www.eren.doe.gov/buildings/documents/pdfs/hvacvolume3finalreport.pdf .

Rubinstein, F., S. Johnson, and P. Pettler. 2000. “IBECS: An Integrated Building Environmental Communications System - It's Not Your Father's Network”, Proc. ACEEE Summer Study on Energy Efficiency in Buildings. Pacific Grove, California, American Council for an Energy Efficient Economy, Washington DC.

Santos, J.J. and E.L. Brightbill, 2002, “Five Principles to Specify Controls By,” HPAC Engineering, April, pp. 11-12.

Schell, M.B., S.C. Turner, and R.O. Shim, 1998, "Application of CO2-based Demand Controlled Ventilation Using ASHRAE Standard 62: Optimizing Energy Use and Ventilation," ASHRAE Transations, TO-98-21-1, pp. 1213-1225. Available at: http://www.carriercommercial.com/pdf_files/public/c0005020.pdf .

Schell and Int-Hout. 2001. "Demand Control Ventilation Using $\mathrm{CO}_{2}$," ASHRAE Journal, February, pp.1829.

Seem, J., J.M. House, and R.H. Monroe. 1999. On-Line Monitoring and Fault Detection, ASHRAE Journal, Vol. 41, No. 7, pp. 21 - 26.

Shockman, C. and M.A. Piette. 2000. "Innovation Adoption Processes for Third Party Property Managers,” Proceedings of the 2000 ACEEE Summer Study on Energy Efficiency in Buildings. Pacific Grove, California, American Council for an Energy Efficient Economy, Washington DC.

Sinclair, K. 2003. “Repackaging the Large Building Automation Industry,” Engineered Systems, February.

Smith, R. G. 1980. The Contract Net Protocol: High-Level Communication and Control in a Distributed Problem Solver, IEEE Trans. Comput. Vol. C-29, No. 12, 1104-1113.

Stallard, L. A. 1989. Model Based Expert System for Failure Detection and Identification of Household Refrigerators. Master's Thesis, School of Mechanical Engineering, Purdue University, Purdue, Indiana.

Stylianou, M. 1997. “Classification Functions to Chiller Fault Detection and Diagnosis.” ASHRAE Transactions Vol. 103, Pt. 1, pp. 645 - 648.

Stylianou, M. and D. Nikanpour. 1996. "Performance Monitoring, Fault Detection, and Diagnosis of Reciprocating Chillers.” ASHRAE Transactions, Vol. 102, Pt. 1, pp. 615-627.

Taylor, Z.T. and R.G. Pratt. 1989. Description of Electric Energy Use in Commercial Buildings in the Pacific Northwest. DOE/BP-13795, prepared by Pacific Northwest National Laboratory for the Bonneville Power Administration, Portland, Oregon. 
Taylor, Z.T. 1992. Description of Electric Energy Use in Commercial Buildings in the Pacific Norhtwest: 1992 Supplement. DOE/BP-1958, prepared by Pacific Northwest National Laboratory for the Bonneville Power Administration, Portland, Oregon.

Thomas, S. 2001. “Our Industry Should Support New Division 17,” AutomatedBuildings.com, November. Downloaded from: http://www.automatedbuildings.com/news/nov01/art/jcist/jcist.htm .

Torcellini, Paul; R Judkoff, S. Hayter. 2002. “Zion National Park Visitor Center: Significant Energy Savings Achieved through a Whole-Building Design Process,” ACEEE Summer Study on Energy Efficiency in Buildings, Pacific Grove, CA, August, American Council for an Energy Efficient Economy, Washington D.C.

Turk, B.H., J.T. Brown, K. Geisling-Sobotka, D.A. Froehlich, D.T. Grimsrud, J. Harrison, J.F. Koonce, R.J. Prill, and K.L. Revzan. 1987. "Indoor Air Quality and Ventilation Measurements in 38 Pacific Northwest Commercial Buildings,” LBL-22315, Lawrence Berkeley National Laboratory, Berkeley, California.

Turner, D., D. Claridge, S. Deng, S. Cho, M. Liu, T. Hagge, C. Darnell, and H. Bruner. 2001. "Persistence of Saving Obtained from Continuous Commissioning", Proc. National Conference on Building Commissioning.

Tutsui, H. and K. Kamimura. 1996. "Chiller Condition Monitoring Using Topological Case-Based Modeling.” ASHRAE Transactions, Vol. 102, Pt. 1, pp. 641-648.

U.S. Department of Energy (US DOE) and Portland Energy Conservation, Inc. (PECI). 1998. "Model Commissioning Plan and Guide Commissioning Specifications, Version 2.05,” Portland Energy Conservation Inc., Portland, Oregon.

Von Neida, B., D. Maniccia and A. Tweed. 2000. “An Analysis of the Energy and Cost Savings Potential of Occupancy Sensors for Commercial Lighting Systems,” Proc. Illuminating Engineering Society of North America Annual Conference, pp. 433-459.

Wagner, J. and R. Shoureshi. 1992. Failure Detection Diagnostics for Thermofluid Systems. Journal of Dynamic Systems, Measurement, and Control, Vol. 114, No. 4, pp. 699-706.

Warwick, M. W., J. De Steese, J. Dagle, D. Jarrell, M. Weimar, N. Lu, and S. Arey. 2003. “Security Benefits of Renewable Generation: A Case Study, Pacific Northwest National Laboratory, Richland, Washington 99352

Westphalen, D. and S. Koszalinski. 1999. "Energy Consumption Characteristics of Commercial Building HVAC Systems - Volume II: Thermal Distribution, Auxiliary Equipment, and Ventilation,” Final Report by Arthur D. Little, Inc. to the U.S. Department of Energy, Office of Building Technology, State and Community Programs, October. Available at: http://www.eren.doe.gov/buildings/documents/pdfs/comhvac.pdf.

Westphalen, D. and S. Koszalinski, 2001, "Energy Consumption Characteristics of Commercial Building HVAC Systems - Volume I: Chillers, Refrigerant Compressors, and Heating Systems,” Final Report by Arthur D. Little, Inc. to US Department of Energy, Office of Building Technology, State and Community Programs, April. Available at: http://www.eren.doe.gov/buildings/documents/pdfs/hvacvolume1finalreport.pdf .

Wheeler, G. 1994. "Performance of Energy Management Systems,” ACEEE Summer Study on Energy Efficiency in Buildings. Pacific Grove, CA, August, American Council for an Energy Efficient Economy, Washington D.C

Willsky, A.S. 1976. “A Survey of Design Methods for Failure Detection in Dynamic Systems.” Automatica, Vol. 29, pp. $601-611$. 
Wortman, D.N., E.A. Evans, F. Porter, A.M. Hatcher. 1996. “An Innovative Approach to Impact Evaluation of Energy Management System Incentive Programs,” ACEEE Summer Study on Energy Efficiency in Buildings. Pacific Grove, CA, August, American Council for an Energy Efficient Economy, Washington D.C.

Wyon, D. 2000. “Enhancing Productivity While Reducing Energy Use in Buildings,” Paper presented at the E-Vision 2000 Conference, Washington, DC, 11-13 October.

Zheng, G. R. and Zaheer-Uddin, M. 1996. “Optimization of thermal processes in a variable air volume HVAC system,” Energy, 21(5):407-420, 1996. 


\section{APpendix A}

\section{LEAD AND CONTRIBUTING AUTHORS}





\section{APPENDIX A}

\section{LEAD AND CONTRIBUTING AUTHORS}

The Market for Advanced Controls and Sensors

Kurt W. Roth, TIAX, (Lead)

Sephir D. Hamilton

Karen R. Benedek

\section{Current Applications and}

Strategies for New Applications

Paul A. Torcellini, NREL, (Lead)

Jeff Christian, ORNL

Michael Deru, NREL

Steve Emmerich, NIST

Brent Griffith, NREL

Philip Haves, LBNL

Michael Kintner-Meyer, PNNL

Bernie Lesieutre, LBNL

Nicholas Long, NREL

Chris Marnay, LBNL

Andrew Persily, NIST

Edward Wolfrum, NREL

\section{Sensor and Controls}

Mike Brambley, PNNL (Lead)

Francis Rubinstein, LBNL

William Healy, NIST

Paul Ewing, ORNL
Jim Hardy, ORNL

Michael Kintner-Meyer, PNNL

Srinivas Katipamula, PNNL

Networking, Security, and

Protocols and Standards

D.G. Holmberg, NIST (Lead)

S. Treado, NIST

D. Chassin, PNNL

F. Rubinstein, LBL

D. Watson, LBL

Automated Diagnostics, Performance Monitoring, Commissioning, Optimal Control and Tools

Philip Haves, LBNL (Lead)

Srinivas Katipamula, PNNL

Mary Ann Piette, LBNL

Jeffrey Schein, NIST

Michael Wetter, LBNL

Natascha Castro, NIST

Steve Bushby, NIST

Roger Kisner, ORNL

Michael Deru, NREL 


\section{APPENDIX B}

\section{ENERGY SAVINGS IMPACT ESTIMATE CALCULATIONS}





\section{APPENDIX B}

\section{ENERGY SAVINGS IMPACT ESTIMATE CALCULATIONS}

\section{B.1 Methodology}

The estimates of market size, market penetration, and potential energy savings of emerging energyefficient technologies come from a six-step methodology:

1. Establish an upper bound of the technical (potential) market size based on the total applicable commercial building floorspace in the United States (i.e., subtracting the floorspace already served by the technology).

2. Define the relevant total primary energy consumption impacted by the technology, based on the energy consumed by the relevant end-uses (HVAC, lighting, etc.) and the technical market size.

3. Estimate the average unit energy savings for the technology for major end-uses, as a percentage of average energy consumption.

4. Calculate the simple payback period (SPP) for the technology based on estimates of installed cost, energy cost savings, and operating cost impact.

5. Estimate the market-achievable penetration (in percent) from the market penetration curves (Figure B.1) based on the calculated SPP.

6. Multiply the market penetration by the relevant primary energy consumption to get the marketachievable energy savings of the technology.

All market-penetration estimates have a substantial degree of uncertainty, which depends on the complexity, features, and benefits of the product modeled, as well as the specific market(s) targeted by the product. For example, the market potential for a specific technology applied to a well-defined existing product, e.g., a specific manufacturer's room air-conditioner product, has less uncertainty than the market penetration estimate for a more broadly defined classes of products, such as packaged rooftop air-conditioners. Different advanced control technologies, however, have significantly higher levels of uncertainty because they are complex systems incorporating a wide range of discrete products or, in the case of commissioning, services. Moreover, as discussed in Section 1.5, many non-financial barriers play a key role in deciding the market success - or failure - of building controls.

Several techniques and methods have been used to predict market penetration of new or emerging technologies. Kastovich et al. (1982) and MTI (1980) both developed market acceptance curves for heat pump equipment for both new and replacement commercial markets. Princeton Synergetics developed the stochastic market penetration model (MPM) for building envelope and end-use products based on payback, risk, cost of competing products, and variability of payback (Princeton Synergistics 1995). This 
paper uses the A market curve for energy-efficient technologies in commercial buildings, which yields an estimated market penetration based on simple payback period (see Figure B.1). The A curve reflects information gathered from field interviews, consumer surveys, and market data on adoption of efficient technologies. They should be viewed as providing guidance on basic trends rather than being quantitatively precise.

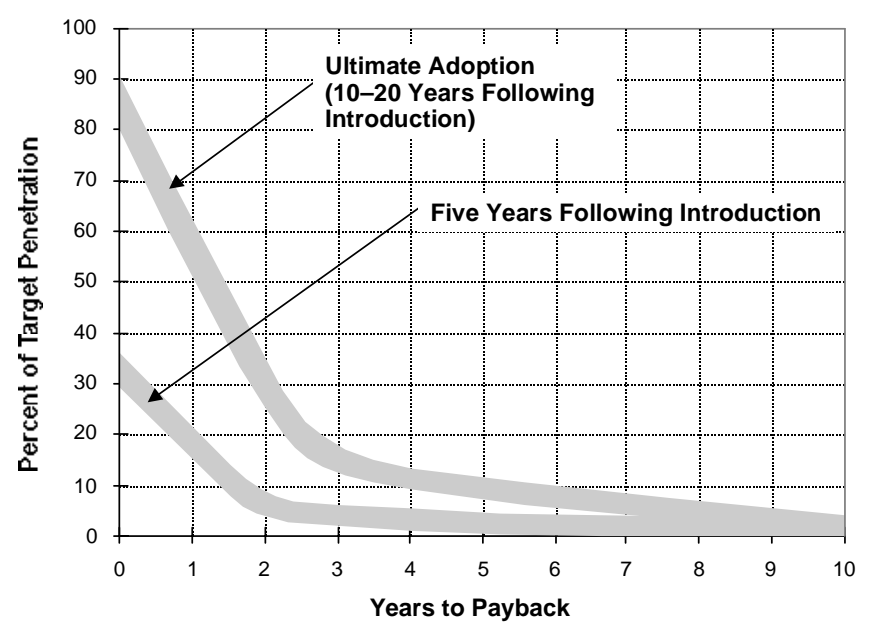

Figure B.1. A Market Penetration Curves

\section{B.2 Energy Prices and Demand Charge Impact}

Energy prices lie at the heart of SPP calculations, as they are needed to translate energy savings into annual energy cost savings. Historically, energy prices have fluctuated over time, which can complicate SPP calculations (see Figure B.2; note, these are nominal prices, i.e., not adjusted for inflation) while also exhibiting substantial geographical and seasonal variations. However, nominal gas and electric prices in the United States have stabilized somewhat since the 1980s and the current average electric price in the commercial buildings sector lies somewhere between seven and eight cents per kilowatt-hour (kWh). The average natural gas rate has fluctuated between five and six dollars per million-BTUs. The most recent data from the Energy Information Administration (EIAa November 2003) shows average electric rates near $\$ 0.08 / \mathrm{kWh}$ and natural gas prices close to $\$ 6 / \mathrm{MMBtu}$ and those values are used for the SPP calculations. 
Average U.S. Electric Retail Prices

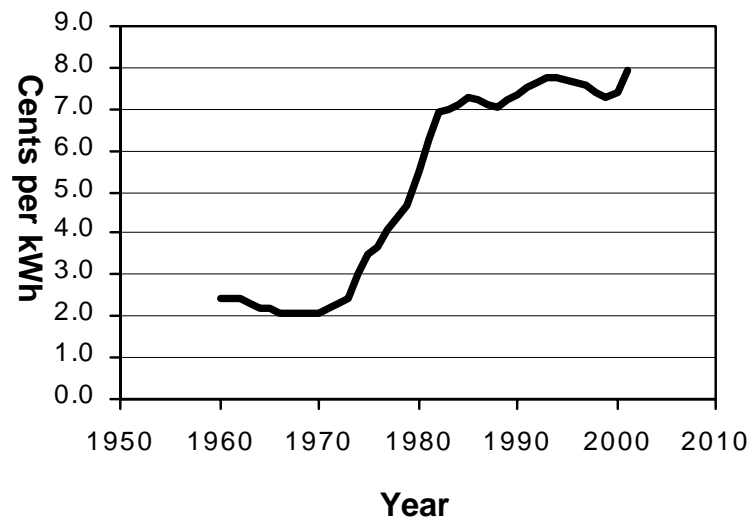

Average U.S. Gas Retail Prices

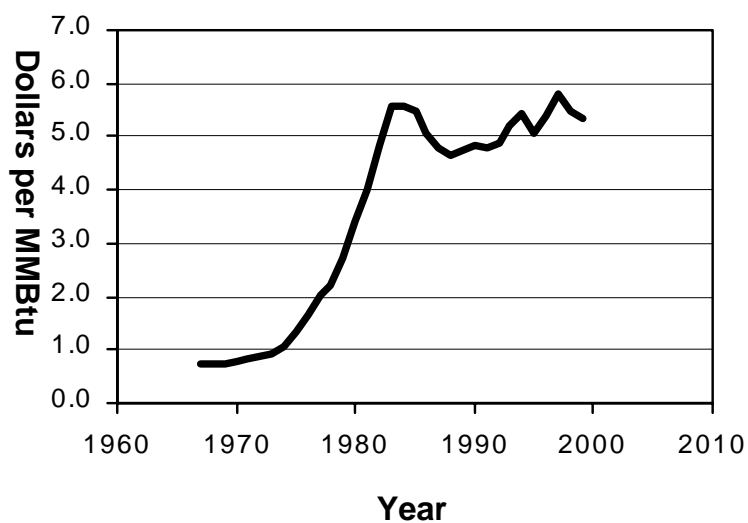

Figure B.2. Average Commercial Electric and Gas Prices in the United States (nominal; from EIA 2003)

For most commercial buildings, the electric price consists of a usage component $(\$ / \mathrm{kWh})$ and a demand charges (\$ per peak kW). Demand charges vary greatly from utility to utility, so the impact of control approaches that reduces peak demand on cost savings varies as well. A review of electric rate structures in six major U.S. cities (Chicago, Los Angeles, Miami, New York, Phoenix, and San Francisco) for both small and large commercial customers found that demand charges are common, especially for larger customers. The magnitude of the demand charges varies by utility, typically in the range from $\$ 5-\$ 15 / \mathrm{kW}$ of peak demand during summer months, with smaller demand charges during the winter). Based on this, a typical demand charge in the United States equals approximately $\$ 10 / \mathrm{kW}$ during the summer and $\$ 5 / \mathrm{kW}$ during the winter.

A review of building load databases for offices, retail buildings, hospitals, schools, and hotels reveals that load factors ${ }^{1}$ typically lie between 0.35 and 0.45 . Using 0.4 as a typical load factor combined with a $\$ 10 / \mathrm{kW}$ demand charge and a $\$ 0.08 / \mathrm{kWh}$ total charge yields the following per-kWh monthly bill:

$$
\begin{aligned}
& \text { Monthly electricity cost (per kWh) }=\$ 0.08 \\
& =\$ 10 / \mathrm{kW} \times 0.00336^{2} \mathrm{~kW} / \mathrm{kWh}+\$ 0.0464 / \mathrm{kWh} .
\end{aligned}
$$

In this case, the ratio of the demand charge (\$0.0336) to the total charge $(\$ 0.08)$ is about $0.4^{3}$. That is, on average, peak demand charges account for roughly $40 \%$ of total electricity expenditures and a $1 \%$ reduction in peak demand reduces annual electricity expenditures by 0.4 . This indicates that building

\footnotetext{
${ }^{1}$ Load factor equals the electric consumption $(\mathrm{kWh})$ in any given time period divided by the product of the peak load $(\mathrm{kW})$ and the number of hours in the time period.

${ }^{2} 0.00336$ equals $1 /$ (load factor[0.4] x hours per month [ 744]) and $\$ 0.0464$ is the difference between $\$ 0.08$ and the product of $\$ 10$ x 0.00336.

${ }^{3}$ This relationship does not hold for extreme peak reductions that will notably impact the load factor, but represents a reasonable approximation for peak reductions of up to $\sim 10 \%$.
} 
controls can add appreciable value by incorporating peak-shaving functionality, such as allowing temperature setpoints to rise during periods of notably high peak demand.

Though addressing peak energy use can yield significant dollar savings for individual building owners, the reduction in peak electricity usage can have an even greater impact for the utility system as a whole. Large amounts of additional capacity, standby reserves, and spinning reserves are on hand primarily to meet regional and seasonal peaks in demand. These reserves tend to be more costly and create more pollution than the generating base. Therefore, addressing peak demand can have a much larger impact than simply reducing the demand charge by freeing excess capital and avoiding increased pollution. Increasing the reliability of the power grid is a serious national security issue, which can be greatly enhanced through the large-scale implementation of automated demand side management.

While some utilities offer real-time electric rates, where the rate varies throughout the day according to the cost of electricity supplied to the utility, the trend towards nationwide adoption has slowed with many utilities suspending their existing real-time rate structures. Kammerud et al. (1996) showed that upwards of 20\% annual energy cost savings could be achieved by effectively using an EMCS to optimize system operation based on real-time pricing in a San Francisco hotel. In light of the slowed adoption of real-time pricing, however, these savings are unlikely to be realized, at least in the short term. 
PNNL-15149

\section{Distribution}

No. of

\section{Copies}

\section{OFFSITE}

Don Anderson

ICF Consulting Inc

9300 Lee Highway

Fairfax, VA 22301

Karen Benedek

TIAX, LLC

15 Acorn Park

Cambridge, MA 02140-2328

David Bornside

Siemens Building Technologies

1000 Deerfield Parkway

Buffalo Grove, IL 60089

Joseph Borowiec

NYSERDA

Building R\&D

17 Columbia Circle

Albany, NY 12203

Martha Brook

California Energy Commission

MS-42

1516 Ninth Street

Sacramento, CA 95814

Karl Brown

California Institute for Energy Efficiency

1333 Broadway Suite 240

Oakland, CA 94612

Steven Bushby

NIST

Building Environment Division (863)

100 Bureau Drive, Stop 8631

Gaithersburg, MD 20899-8631

Ronald Caffrey

BCS Partners

2008 Carolina Circle N.E.

St. Petersburg, FL 33703
Michael Chapman

Naval Facilities Engineering Command

Design Policy/Architecture

1322 Patterson Avenue, SE

Washington Navy Yard

Washington, DC 20374-5065

David Claridge

Texas A\&M University

Department of Mechanical Engineering

College Station, TX 77843-3123

Dru Crawley

U.S. Department of Energy

1000 Indepedence Ave, SW, EE-2J

Washington, DC 20585

Paul Ewing

RF \& Microwave Systems Group

Oak Ridge National Laboratory

One Bethel Valley Road

Building 3500 MS 6006 Room B18

PO Box 2008

Oak Ridge, TN 37831-6006

Kevin Finnegan

Battery Park City Authority

One World Financial Center

New York, NY 10281-1097

Theo Frutiger

Siemens Building Technologies

Siemens SBT HVAC Products

1000 Deerfield Parkway

Buffalo Grove, IL 60089

Vijay Gupta

U.S. General Services Administration

Office of the Chief Architect

1800 F Street, NW

Washington, DC 20405

20 David Hansen

U.S. Department of Energy

1000 Independence Ave, SW, EE-2J

Washington, DC 20585

Tom Hartman

The Hartman Company

9905 39th Drive NE

Marysville, WA 98270

Distr. 1 
PNNL-15149

Philip Haves

Lawrence Berkeley National Laboratory

Building 90, MS 90-3074

1 Cyclotron Road

Berkeley, CA 94720

5

David Holmberg

NIST

Building Environment Division (863)

100 Bureau Drive, Stop 8631

Gaithersburg, MD 20899-8631

John House

Iowa Energy Center

Suite 124

2521 Elwood Drive

Ames, Iowa 50010-8263

Steve Karg

Lithonia Lighting

One Lithonia Way

Building 1

Decatur, GA 30035

Roger Kisner

Oak Ridge National Laboratory

One Bethel Valley Road

Building 37831 MS 6004

P.O. Box 2008

Oak Ridge, TN 37831-6004

Ron Lewis

U.S. Department of Energy

1000 Independence Ave, SW, EE-2J

Washington, DC 20585

Eric Lightner

U.S. Department of Energy

Office of Electric Transmission and

Distribution

1000 Independence Ave, SW, EE-2D

Washington, DC 20585

Vivian Loftness

Carnegie Mellon University

Architecture Department

Head of the School of Architecture

500 Forbes Avenue

4919 Frew St. Room 201

Pittsburgh, PA 15213

Tom Lohner

Teng \& Associates

4050 Innslake Drive

Suite 300

Glen Allen, VA 23060-3327

John McKissack

The Kele Companies

3300 Brother Boulevard

Memphis, TN 38133

Terrence McMahon

BCS Partners

135 Fort Lee Road

Leonia, NY 07605

Mark Myers

United Technologies Research Center

411 Silver Lane

Mail Stop 129-85

East Hartford, CT 06108

$5 \quad$ Kurt Roth

TIAX, LLC

15 Acorn Park

Cambridge, MA 02140-2328

John Ryan

U.S. Department of Energy

1000 Independence Ave, SW, EE-2J

Washington, DC 20585

Harvey Sachs

ACEEE

Buildings Programs

1001 Connecticut Ave NW, Suite 801

Washington, DC 20036

Michael K. Sahm

United Technologies Research Center

411 Silver Lane

Mail Stop 129-65

East Hartford, CT 06108

Jeffrey Schein

NIST

Building Environment Division (863)

100 Bureau Drive, Stop 8631

Gaithersburg, MD 20899-8631

John Seem

Johnson Controls

507 East Michigan Street

Mail Stop M36

Milwaulkee, WI 53202

Sandy Smith

ACEEE

Buildings Programs

1001 Connecticut Ave NW, Suite 801

Washington, DC 20036

Distr. 2 
PNNL-15149

Mike Stein

Mike Stein Associates

40 Alize Drive

Kinnelon, NJ 07504

Randal Stites

Hines GS Properties, Inc.

901 D. Street, SW

Box 18, Suite 290

Washington, DC 20024-2169

$5 \quad$ Paul Torcellini

National Renewable Energy Laboratory

1617 Cole Blvd

Mail Stop 2722

Golden, CO 80401-3393

Stephen Treado

NIST

Building Environment Division (863)

1000 Bureau Drive, Stop 8631

Gaithersburg, MD 20899-8631

Martin Weiland

ASHRAE

1828 L St. N.W., Suite 906

Washington, DC 20036-5104

Robert Williams

Continental Automated Buildings Association

(CABA)

4200 NW 74th Court

P.O. Box 901525

Kansas City, MO 64151
No. of

Copies

ONSITE

29 Pacific Northwest National Laboratory

Michael Brambley (5)

K5-16

Sean McDonald (20)

BWO

Marylyn Placet

BWO

Theresa Gilbride

BPO

Information Release Office (2)

P8-55

Distr. 3 